Portland State University

PDXScholar

Summer 7-16-2015

\title{
A Critical Race Analysis of Travel for Transformation: Pedagogy for the Privileged or Vehicle for Socio- Cultural Transformation?
}

James Arthur Gambrell

Portland State University

Follow this and additional works at: https://pdxscholar.library.pdx.edu/open_access_etds

Part of the Bilingual, Multilingual, and Multicultural Education Commons Let us know how access to this document benefits you.

\section{Recommended Citation}

Gambrell, James Arthur, "A Critical Race Analysis of Travel for Transformation: Pedagogy for the Privileged or Vehicle for Socio-Cultural Transformation?" (2015). Dissertations and Theses. Paper 2485. https://doi.org/10.15760/etd.2482

This Dissertation is brought to you for free and open access. It has been accepted for inclusion in Dissertations and Theses by an authorized administrator of PDXScholar. Please contact us if we can make this document more accessible: pdxscholar@pdx.edu. 
A Critical Race Analysis of Travel for Transformation: Pedagogy for the Privileged or Vehicle for Socio-Cultural Transformation?

by

James Arthur Gambrell

A dissertation submitted in partial fulfillment of the requirements for the degree of

Doctor of Education

in

Educational Leadership: Curriculum and Instruction

Dissertation Committee:

Susan Lenski, Chair

Anita Bright

Andy Job

Ethan Johnson

Portland State University

2015 
(C) 2015 James Arthur Gambrell 


\begin{abstract}
Transformative learning theory (TLT) describes the process of reframing discriminative, untenable worldviews with a more inclusive, permeable, and reflective epistemology. Although TLT has been around for more than 50 years, few studies empirically engage critical theoretical frameworks to move beyond personal learning to identify the impacts of transformation on society. Through a critical race theory (CRT) framework, this study analyzed how the participants' socio-cultural identities (race, class, gender, sexuality, and heritage language) impacted the transformative learning of eight study-abroad students from a medium-sized, rural Midwestern university. Focusing on the extent to which the participants experienced the elements of transformative learning during a month-long study-abroad experience in Spain, this project explored what the experience of traveling to an "other" place and experiencing "otherness" had on transformative learning. This qualitative study drew from extensive ethnographic observations, photo analysis, and informal, semi-formal, and focus-group interviews. During the study, the researcher analyzed data to construct codes and categories for further analysis, incorporating multiple member-checks to promote the narratives of the participants. Following data collection, the researcher used constructivist grounded theory to further scrutinize the data searching for salient themes and patterns connected to the research questions. Race and class privilege (or marginalization) had an impact on the study-abroad participants' worldviews and transformative learning. Participants from backgrounds of racial and class privilege experienced transformations that were deeply engaging on a personal
\end{abstract}


level, but were not motivated toward more justified beliefs or action toward others. In contrast, participants from backgrounds of lesser socio-cultural privilege demonstrated increased critical social reflection and transformative outcomes, both personal and social. These findings led the researcher to question if there might be more effective avenues toward critical social transformation than the pedagogy of the privileged of travel for transformation. 


\section{Table of Contents}

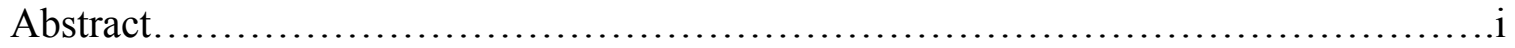

Chapter One: Problem Statement.............................................

Chapter Two: Literature Review $\ldots \ldots \ldots \ldots \ldots \ldots \ldots \ldots \ldots \ldots \ldots \ldots \ldots \ldots \ldots \ldots \ldots \ldots \ldots$

Chapter Three: Methodology...................................................94

Chapter Four: Findings.................................................. 168

Chapter Five: Discussion and Conclusions.....................................403

References...............................................................467

Appendices

Appendix A: Pre-/Post-Surveys and Permission for Use....................485

Appendix B: Human Subjects Approval.................................491 


\section{Chapter One: Problem Statement}

In my early twenties, I embarked on a two-year proselyting mission for my church in Spain. Although the mission experience was designed to be spiritually transformative (which it was), what was most surprising was the socio-political transformation that I underwent. Over the course of two years, the people, language, culture, and Spain itself changed me. This transformation did not happen all at once. Rather, little experiences changed my socio-political perspective. During my first few weeks in Barcelona, I was amazed that a city of four million people shut down at 2:00 p.m. every day so families could go home and eat the big meal of the day together. When I inquired about how this was possible, my Spanish friends replied, "In the United States, you live to work. Here in Spain, we work to live." In addition, I perceived a noticeably smaller gap in the lifestyles between the wealthy and the poor. When I inquired if it bothered anyone that almost everyone I knew lived in a small (even to my working class upbringing) apartment, my Spanish friends answered that their way of life was much more important than acquiring possessions. They maintained that by collectively agreeing to smaller homes, they obtained universal healthcare, wonderful public transportation systems, beautiful parks, and a mandatory month's vacation leave for all workers. These benefits granted them more leisure time to spend with friends and family discussing news, debating politics, having neighborhood parties, and building community.

More than simply loving the culture of Spain, my ideas about the United States began to change. I experienced cognitive dissonance there and reverse culture shock upon return to the United States. I began to question the meta-narratives that the United 
States was a meritocracy, that capitalism was just, and that this society was equitable. In addition, my outlook and behavior became much more reflective, tolerant, and permeable. Several of my North American flat-mates experienced similar socio-political transformations while living in Europe for two years. I had always wondered what made this transformation occur. Years later, I discovered transformative learning theory in a graduate class. Understanding this theory provided me with the vocabulary to explain what I and others have experienced while being immersed in other cultures.

\section{Research problem}

Transformative learning theory (TLT) has become the most widely used model of adult learning in the United States (Cranton \& Taylor, 2012). Transformative learning theory describes the process of reframing discriminative, untenable worldviews with a more inclusive, permeable, and reflective epistemology. TLT is situated within a theoretical framework of a constructivist epistemology, a humanist axiology, and a critical social ontology. Constructivism assumes that no single, objective reality exists. Rather, in both constructivism and TLT, knowledge is what an individual creates, or constructs, in the mind versus what can be scientifically measured, or what others tell us is right or wrong (Fosnot \& Perry, 2005). Transformative learning theory's humanist axiology assumes that humans are inherently good, free, autonomous, and capable of self-actualization (Cranton \& Taylor, 2012). Finally, from a critical social ontology, one comes to understand that large governmental, legal, educational, and social systems have been historically designed to oppress, marginalize, and to reinforce white, male, heterosexist patriarchy (Brookfield, 2012). Consequently, discrimination and unfair, 
biased policies, norms, and practices exist within our educational organizations to an extent that freeing the entire system of inequity will be a slow process if current structural frames remain.

Although transformative learning theory (TLT) has been around for more than 50 years, few studies exist that empirically explore transformation through a socio-cultural lens (Ross, 2010). Furthermore, many scholars assert that Mezirow's (2000) version of TLT is overly focused on individual transformation and does not deal directly with sociocultural transformation, causing the theory to be overly individualistic, gendered, raced (White-centered), self-centered rather than earth-centered, and North Americancentered. I label this push among TLT scholars for greater social action critical social transformative learning theory (critical social TLT).

Critical social TLT fosters perspective transformation that helps individuals identify dominant ideology and hegemony, unmask power, and then develop agency to oppose ideological forces that work against societal interests. Moreover, because transformative learning theory is not a political theory, it makes a poor framework for analyzing political systems, institutions, and organizations that impact transformation. Therefore, another framework, such as critical race theory, can be used to critique policies, system, and institutions that affect educators' capacity to carry out transformative learning practices. Critical race theory is a form of oppositional scholarship that exposes racism as normal; uncovers interest convergence; deconstructs the myths of colorblindness within educational institutions; promotes the voices and narratives of people of color; and exposes White, middle-class values as normative. 
Another burgeoning area of research explores the effects of travel, tourism, and study-abroad programs on transformation of cultural worldview (Coghlan \& Gooch, 2011; Cordero \& Rodriguez, 2009; Falk, Ballantine, Packer, \& Brinckerhoff, 2012; Morgan, 2010). Furthermore, researchers are investigating how critical social TLT intersects with travel, volunteer tourism, and study-abroad programs (Pritchard, Morgan, \& Ateljevic, 2011; Ross, 2010). Travel for transformation uniquely opens a person to transformative learning because it takes an individual out of daily routines and positions the learner into a state of geographic, linguistic, and cultural "otherness."

\section{Purpose}

In order to expand research that lies at the intersection of critical social TLT and transformation for travel, the purpose of this study was to understand how individuals experienced transformative learning when traveling to cultures other than their own. In order to understand travel for transformation, I describe to what extent eight students from a medium-sized, rural Midwestern university experienced the elements of transformative learning during a month-long study-abroad experience in Spain. In addition, this project explored what the experience of traveling to an "other" place and experiencing "otherness" had on transformative learning. Finally, this research examined through a critical race theory paradigm how each participant's racial, economic, gender, and cultural identity impacted her or his worldview and unpacked how these social variables affect the transformation process. 


\section{Research Questions}

In order to achieve the purpose above, the research in this project will be guided by the following questions:

1. To what extent do eight students experience elements of transformative learning during a month-long study-abroad program in Spain?

2. What role did encounters with cultural and linguistic "otherness" play in the transformative learning (or lack thereof) of each participant?

3. What is each student's racial, economic, gender, and cultural identity? How does each student's identity impact her or his worldview and her or his transformations (or lack thereof) during the study-abroad program?

\section{Methods}

A qualitative research methodology incorporating biological narratives, photo/video analysis, extensive ethnographic observations, surveys, and informal, semiformal, and focus group interviews was used in this study. The data collection of this project borrowed heavily from Glaser's (2012) idea that “all is (sic) data:" everything the researcher sees, hears, or experiences contextualized the interviews, observations, and surveys. For data analysis, however, Charmaz's (2003) constructivist grounded theory was used. Constructivist grounded theory presupposes multiple social realities, mutual creation of knowledge from both researcher and participant, and seeks to understand the participants from extensive collection of rich data with thick description. The participants under this construct become co-participative storytellers with the researcher 
in the generation of codes, patterns, and overarching themes. Following the study, data were scrutinized searching for salient themes, patterns, or other relevant information.

\section{Significance of the Research Problem}

A major benefit to this research project was to study what elements of studyabroad programs have the potential for transformative learning. While many colleges and universities implement study-abroad programs with the underlying assumption that they are transformative, little empirical research has been done to examine the elements of travel that contribute to transformation (Ross, 2010). Even fewer studies have been carried out to explore the socio-cultural variables of race, gender, sexuality, class, and (dis)ability and how they impact travel for transformation (Pritchard, Morgan, \& Ateljevic, 2011). This research project was an initial attempt to generate theory pertaining travel for transformation through a socio-cultural lens. The results of this study and others like it could assist universities in deciding whether study-abroad programs are a pedagogy for the privileged few who can undertake such journeys or a vehicle for critical socio-cultural transformation. Consequently, university programs can decide how much energy, time, and funding to allocate to study-abroad programs in the future.

In a broader context, the primary goal of transformative learning theory is to help students, teachers, and administrators overcome distorted, biased, or destructive worldviews. Many students are underserved, undervalued, and deprived of their voice in classrooms. Students who are left out of the learning process, tend to suffer from poor learning outcomes, resulting in either poor test results or from dropping out of school 
altogether (Darling-Hammond, 2007). Consequently, as those occupying positions of privilege move toward more inclusive frames of reference, marginalized students will have the opportunity to be more fully engaged in the learning process. Furthermore, as educators learn to help sidelined students participate more fully, student outcomes will improve (Johnson \& Johnson, 1989; Kagan, 1994). Closing the racial and socioeconomic achievement gap in primary and secondary educational institutions will hopefully instill the desire for more students to be present in the transformative learning process taking place in post-secondary classrooms. However, if nothing else, the transformative learning values of promoting cooperation rather than competition; incorporating discussion, inclusion, and participation; and encouraging openness to different beliefs are valuable social-justice goals in and of themselves.

\section{Summary}

Transformative learning theory describes the process of reframing discriminative, untenable worldviews with a more inclusive, permeable, and reflective epistemology. Although transformative learning theory (TLT) has been around for more than 50 years, few studies exist that empirically explore transformation through a sociocultural lens. This study situated TLT within both sociocultural theory and critical race theory (CRT). The purpose of this study was to describe to what extent eight students from a medium-sized, rural Midwestern university experienced the elements of transformative learning during a month-long study-abroad experience in Spain. In addition, this study explored what the experience of traveling to an "other" place and experiencing "otherness" had on transformative learning. Finally, this research examined 
how each participant's racial, economic, gender, and cultural identity impacted her or his worldview and unpacked how these social variables affected the transformation process. These research questions were answered through qualitative research methodology incorporating biological narratives, photo/video analysis, extensive ethnographic observations, surveys, and informal, semi-formal, and focus group interviews. The data were analyzed using Charmaz's (2003) constructivist grounded theory. Constructivist grounded theory presupposes multiple social realities, mutual creation of knowledge from both researcher and participant, and seeks to understand the participants from extensive collection of rich data with thick description. The participants under this construct became co-participative storytellers with the researcher in the generation of codes, patterns, and overarching themes. Following the study, data were scrutinized searching for salient themes, patterns, or other relevant information. 


\section{Chapter Two: Literature Review}

The purpose of this chapter is to establish a theoretical foundation from existing academic literature on travel for critical social transformation. E. W. Taylor ${ }^{1}$ and Snyder (2012) state that literature reviews "synthesize significant findings, help identify areas of concern and questions yet to be explored, and potentially provoke the status quo, challenging the field to question or rethink what is often unquestioned" (p. 37). This chapter begins with a brief introduction of transformative learning theory (TLT). Following, I provide a history of TLT to contextualize the theory in its past while also providing possible outcomes for moving forward. Next, I provide a theoretical framework that situates TLT within the constructs of a constructivist epistemology, humanist axiology, and critical social ontology. Part of the theoretical framework is a call by scholars to push the framework toward critical social action including an explanation of how critical race theory provides an excellent framework for accomplishing this objective. Subsequently, I discuss the process of transformative learning incorporating the concepts of cognitive dissonance, disorienting dilemmas, critical reflection, and reflective discourse as avenues toward transformation. Finally, I end the chapter with recent studies that explore whether travel can be used as a tool for critical social transformative learning.

${ }^{1}$ There are two Edward Taylors and a Kathleen Taylor cited in this literature review. To maintain consistency and ease of reading, Edward W. Taylor will be cited E. W. Taylor, Edward Taylor will be E. Taylor, and Kathleen Taylor will be K. Taylor for in-text citations. For parenthetic references, I refer the reader to the reference list. 
Transformative learning theory describes the process of reframing discriminative, untenable worldviews with a more inclusive, permeable, rational, and reflective epistemology (Ettling, 2006; Johnson-Bailey \& Alfred, 2006; Merriam, 2004; Mezirow, 1996, 1997, 1998, 2000, 2004, 2012; O'Sullivan, 1999). Mezirow (2000) explained that we transform our frames of reference through critical reflection on the assumptions upon which our interpretations, beliefs, habits of mind, or points of view are based.

Transformation commences with cognitive dissonance, when a person is confronted with an idea or experience that contradicts a prior underlying assumption of a personal belief system (Ettling, 2006; Mezirow, 1998, 2002). Cognitive dissonance then serves as a catalyst for critical reflection (Brookfield, 1998, 2002; Ettling, 2006), which in turn leads to a "disorienting dilemma," requiring a reordering of epistemological assumptions and causing a change in beliefs and behaviors (Mezirow, 2000, p.22).

Transformative learning theory (TLT) posits that individuals construct knowledge through informal logic, beliefs, natural dispositions, and skills from experiences, parents, culture, and education before arriving at adulthood (Mezirow, 1998). Transformative learning theory's "focus is on how we learn to negotiate and act on our own purposes, values, feelings, and meanings rather than those we have uncritically assimilated from others--to gain greater control over our lives as socially responsible, clear-thinking decision makers" (Mezirow, 2012, p. 76). In order to become rational thinkers, individuals must become critically reflective of their own and others' underlying intentions, values, beliefs, and feelings (Mezirow, 1997). Before individuals can come to a new or revised interpretation, they must understand their past experiences. Therefore, 
Mezirow explained, "central to this process is helping learners to critically reflect on, appropriately validate, and effectively act on their (and other's) beliefs, interpretations, values, feelings, and ways of thinking" (Mezirow, 2012, p. 89). Coming to understand one's own views makes the transformation process a "deeply engaged process" (Ettling, 2006, p. 63) that is "intensely threatening" (Mezirow, 2000, p. 6) because one has to rationally justify or relinquish assumptions that were previous ways of coping with others' behavior and the outside world. Mezirow (1996) postulated that coming to rational and justified ways of knowing requires a person to be critically reflective of experience, able to manage emotions, empathic to others, and capable of assessing reasons underlying personal belief systems and actions based on those beliefs (Mezirow, 1996). Mezirow (2012) further stated:

Transformative learning refers to the process by which we transform our takenfor-granted frames of reference... to make them more inclusive, discriminating, open, emotionally capable of change, and reflective so they may generate beliefs and opinions that will prove more true or justified to guide action. (p. 76) Put another way, transformation is a process where individuals come to a conclusion that a previous paradigm is incorrect and then change their beliefs and actions to represent a better way of seeing and acting in the world (Mezirow, 2000). However, Tisdell (2012) cautions that educators often overuse or misuse the term transformative so that any form of learning is called transformative, rendering the word transformative nearly meaningless. While definitions of transformation (and how the process unfolds) vary among TLT theorists, the outcome is generally similar: "a deep shift in perspective, 
leading to more open, more permeable, and better-justified meaning perspectives" (Cranton \& Taylor, 2012, p. 3; see also, Mezirow, 2000).

As one authentically becomes aware of personal values and underlying assumptions, transformative learning engages a deep structural shift in that person's consciousness and way of acting in the world (Ettling, 2006). According to Brookfield (2012), transformation is one of the most powerful words in the English language; it indicates that something is fundamentally different than what it was before. Transformative learning results in a profound change in worldview leading to a "deep, structural shift in the basic premises of thought, feelings, and actions. It is a shift of consciousness that dramatically alters our way of being in the world (O'Sullivan, 2012, p. 164).

O'Sullivan (2012) further argues that a dramatic shift in consciousness typically involves becoming aware of systems of power: "Such a shift involves an understanding of ourselves and our locations, our relations with other humans and with the natural world; an understanding of the relations of power in the interlocking structures of class, race, and gender" (p. 175). Moreover, as individuals change the way they understand their place in the world, they discover a "more autonomous sense of self characterized by agency, choice, reflection, and rationality" (Taylor \& Elias, 2012, p. 150).

Some transformational changes are epochal-- "a sudden, dramatic, reorienting insight" (Mezirow, 2012, p. 86)--that challenge/change a person's core identity, worldview, or ultra-consciousness. Epochal transformations invite people to live more deeply, cultivate hope, instill in them a desire to act, and emancipate them from 
unsustainable attitudes and behaviors (Tisdell, 2012). Once a person has had an epochal change she or he will never go back to the old way of seeing the world (Mezirow, 2012; O’Sullivan 1999, 2012).

In 2010, I experienced an epochal change when I enrolled in Dr. Maggie Barber's class at the University of Utah ${ }^{2}$. There, I was immersed in the literature of critical theorists such as Ladson-Billings, Delgado-Gaitan, Delgado-Bernal, Alemán, Villalpando, Freire, O'Sullivan, Cochran-Smith, Brookfield, and Mezirow. The articles we read for class clarified complex theories and ideas with which I identified, but never had the capacity or vocabulary to communicate. Dr. Barber created a classroom environment where the opinions and experiences of the class were valued, and we were encouraged to consistently apply the articles to our respective work and school organizations. Having adopted conservative family and religious culture values from my childhood, many of the articles/class topics created extreme cognitive dissonance for me. However, deep down in my subconscious, I felt and acknowledged the importance of the social justice topics. Over the course of 15 weeks, my attitudes, worldview, behavior, and view of my place in the world changed dramatically.

From the articles, my classmates, and Dr. Barber, I came to understand that as a White, Christian, heterosexual male, I must acknowledge that educational, financial, linguistic, and many other organizational systems are designed to give me a position of

\footnotetext{
${ }^{2}$ The constructivist grounded theory approach used in this dissertation assumes multiple realities and subjectivism. Because this requires the researcher to be transparent about (and check) personal biases, it is typical for the researcher to include personal reflections throughout the research process in order to form mutuality between researcher and participants and author and reader.
} 
privilege over other races, ethnicities, nationalities, sexual orientations, belief systems, cultures, genders, transgender individuals, classes, and those living in generational and extreme poverty. Notwithstanding my privilege, I choose to actively seek methods to create a more inclusive worldview and eradicate forms of inequity that are within my sphere of influence. I became conscious of my role as an observer of marginalization and have came to understand that action is required of those who observe injustice (DelgadoGaitan, 1993; Kezar, Carducci, \& Contreras-McGavin, 2006; Ladson-Billings \& Donner, 2005). My core identity, my sense of self, and how I saw the world was irreversibly changed in that graduate class. Epochal changes like this are rare over the course of one's life (Tisdell, 2012).

While some transformations alter our very being, our core, our sense of self, and how we define ourselves to the world, there are also incremental transformations that happen often--even daily--that transform our frames of reference. Mezirow (2012) explained that "transformations in habit of mind may be ... incremental, involving a progressive series of transformations in related points of view that culminate in a transformation in habit of mind" (Mezirow, 2012, p. 86). Because epochal transformations are rare over the course of an individual's life, Tisdell (2012) cautions adult educators from constantly going after these "peak experiences" (p. 31) in their students. She states that many incremental changes, over time, can lead to equally as poignant of a transformation, but the transformation may happen much later than the semester in which the class is held. 
A personal example of incremental transformative change comes from 14 years of knowing my wife, Catherine. Catherine is aware of power structures, extremely sensitive about the word choices she uses, and a constant advocate for the marginalized. When we were first dating, I sometimes used gendered, racialized, or homophobic speech (which, at the time, I didn't understand or know to be discriminatory as they were a reproduction of the culture in which I was immersed). Catherine would point out that some people might be offended with my word choice. I would often counter that she spent too much time being politically correct. Next, I would say I wasn't trying to hurt anybody and people were overly sensitive if they were offended by my speech patterns. Additionally, I felt that I was not a bigot and thought people would know that if they knew me. She would counter from a personal point-of-view, "As a woman, I find the term 'mailman' offensive because it genders a whole profession." Slowly, I changed my speech habits around her to keep peace in our relationship. Over time, I came to understand that my old speech patterns were discriminatory and offensive. This incremental transformation happened so imperceptibly and over 14 years, but my attitudes and behavior have completely changed regarding racist, homophobic, sexist, heterosexist, ableist, and cigenderist speech. I cannot pinpoint a moment where I had an epiphany, but the change over time has been significant and irreversible.

\section{Historical Background of TLT}

In order to understand TLT, it is essential to contextualize it within its historical underpinnings and to contextualize it within the social movements that were happening in the United States as TLT was developed. Baumgartner (2012) gives an excellent history 
of Mezirow's theory 1975-2012, which I use as both an historical background and as a framework for this section. Furthermore, I include readings from other sources to outline the historical development of TLT to augment Baumgartner's article. This history will help the reader understand where TLT comes from, but more importantly, how it should be adapted for the 21 st Century.

Mezirow's 1969 study was a grounded theory study that sought to conceptualize and explain how adults understand things differently from children (Mezirow, 2000). Other educational theorists investigating adult learning at the time were Knowles (andragogy) and Tough (investigated adult self-directed study). Some scholars would argue that Mezirow developed TLT in direct opposition to Knowles' adult learning concept of andragogy, which was a break from the child-centered approach of pedagogy (Cranton \& Taylor, 2012). Most scholars, however, would situate TLT within the greater umbrella of adult learning (Tisdell, 2012). Knowles' (1970) adult learning model of andragogy states that adults are different from children in the way they learn because they are voluntary, self-directed, experiential, and collaborative learners (see also, Cranton \& Taylor, 2012). Furthermore, the educator and the student are more likely to share more in common as co-learners than with an adult/child relationship common in K-12 learning. Likewise, Taylor and Elias (2012) state that most adult educators "are likely to be traveling a developmental trajectory similar to that of our adult learners" ( p. 159). To Mezirow (2012), an adult is someone who is old enough to be responsible for her or his actions, is able to understand power structures, is able to make rational decisions, has 
autonomy to make those decisions, and is able to communicate the reasons behind making said decisions.

According to Baumgartner (2012), Mezirow directly challenged the quantitative methods that were prevalent in the 1960s and 1970s by using grounded theory data analysis. Mezirow primarily studied adult women, which must be understood through the historical context of the feminist/women's movements of the 1960s (Merriam \& Kim, 2012). He studied factors that facilitate or impede women who were returning to college after lapsed time between high school and college (Mezirow, 2000).

During the 1980s Mezirow refined and added depth to his theory--defining, expanding, and refining terms like "perspective transformation," "meaning perspective," and "meaning schemes" (Baumgartner, 2012). Mezirow was greatly influenced during this time by Habermas' use of discourse and dialogic learning and how people need to assess the validity under ideal discourse conditions (Mezirow, 2000, 2012). In addition, reflectivity was greatly stressed due to Habermas' influence during this time (Baumgartner, 2012). It was also during the 1980s that critics started to criticize Mezirow's lack of focus on social change. Up to this point, Mezirow believed (or at least wrote about) transformation of perspective as if that were the end in and of itself (Cranton \& Taylor, 2012). However, critics noted that TLT focused too much on the individual and did not adequately address the social conditions that contributed to the unjustifiable thought processes (Brookfield, 2012; O’Sullivan, 2012). Mezirow countered that the critics only solved socio-cultural problems with collective social action 
and failed to identify the epistemic distortions, which he felt were the root of social inequity (Baumgartner, 2012).

Throughout the 1990s, Mezirow continued to refine his focus on critical reflection (Baumgartner, 2012). He maintained that the highest order of learning - the kind necessary for transformation of belief systems - requires critical discussion, full literacy, the capacity for both objective and subjective reframing, and critical reflection of assumptions (Mezirow, 1996; 1998). According to Mezirow (1998), personal autonomy is required for transformative learning to take place. To be autonomous, a person must have a critically reflective disposition of one's own assumptions and engage effectively in discourse to validate one's beliefs through others who share universal values (Mezirow, 1997). During the 1990s, Mezirow also identified critical reflection of assumptions and critical reflection of self-assumptions. Critical reflection of assumptions led to objective reframing: "critically examining the validity of the concepts, beliefs, feelings or actions being communicated" (Mezirow, 1998, p. 192), while critical reflection of self assumptions led to subjective reframing, which is the application of critical reflection to one's self. Critical reflection of self assumptions can also lead to subjective reframing about one's place in society, institution, or a system.

Critiques in the 1990s came from theorists who stated that Mezirow still did not adequately contextualize or address issues of gender, sexuality, class, and race (O'Sullivan, 1999). In addition, they stated that Mezirow's theory asserted that personal transformation was sufficient, regardless of social transformation. Furthermore, critiques came from postmodernists who argued that Mezirow's focus on a unified self failed to 
explain the multifaceted, complex nature of humans (Baumgartner, 2012). Also, critics stated that Mezirow's theory reflected White, Western values of independence, selfdirection, and human agency, which is not the case for all cultures. Finally, critics argued that the conditions for ideal discourse were never achieved, which made his theory a too messy for real-life situations.

In the 2000s, Mezirow began to address the context of learning, calling for transformative learning that focused on economic, cultural, political, and educational power within cultural and media narratives, systems, organizations, and workplaces (Mezirow, 2000). Merriam (2004) suggested that the psychological and cognitive capabilities of this type of transformative learning required higher levels of cognition than those possible by undergraduate students. Furthermore, she argued that Mezirow's critique of premises or presuppositions pertains to problem posing as distinct from problem solving. Moreover, Merriam claimed that problem posing takes advanced cognitive function because it involves making a taken-for-granted situation problematic and then the student must raise questions regarding its validity. Many psychologists would maintain that this level of critical thought is not possible until a individuals reach their thirties, or even forties (Merriam, 2004). In 2006, Mezirow acknowledged that his theory under-represented the role of intuition and said it might be necessary for transformation outside of one's own consciousness (Baumgartner, 2012).

Throughout the past decade, TLT researchers have been looking beyond Mezirow for other theoretical orientations of transformative learning (Taylor \& Snyder, 2012). For example, empirical research is beginning to indicate that reflective discourse and critical 
reflection may not be as effective in transforming frames of reference as experience (Baumgartner, 2012). In addition, Johnson-Bailey and Alfred (2006; see also, JohnsonBailey, 2012) posit that marginalization may be the more powerful transformer than critical reflection. Additionally, E. W. Taylor (2007) states that studies have shown that context may be the most important variable on transformative learning outcomes and calls for experimental approaches with different sociocultural variables (race, class, gender, sexual orientation, and culture). Moreover, E. W. Taylor and Snyder (2012) found that "few studies explore whether there was something unique about the participants' background, culture, and/or positionality and the experiences in relationship to transformative learning" (p. 40). However, because individuals occupy multiple contexts simultaneously, it is difficult to ascertain the role of the influence of context on transformation within individuals (Baumgartner, 2012; Crenshaw, 2009). Nevertheless, Baumgartner (2012) asserts that despite the difficulty in teasing out the different variables of marginalization, studies that address sociocultural variables still need to be carried out. Research methodologies within TLT are beginning to expand the boundaries of Mezirow's theory and are showing increased sophistication by incorporating longitudinal designs, action research, content analysis of various documentation analysis (emails, journals, portfolios), video recordings, arts-based research, narrative analysis, case studies, content analysis of student writing, group dialogue research, media analysis, and participatory storytelling (Merriam \& Kim, 2012; Taylor \& Snyder, 2012). Furthermore, TLT research is drawing from the knowledge and methods of other fields like psychology, sociology, art, music, dance, archeology, critical theory, geography, 
economy, anthropology, post-modernism, situated cognition, philosophy, and feminism (Cranton \& Taylor, 2012; Falk, Ballantyne, Packer, \& Benckendorff, 2012; Mezirow, 2012; Taylor \& Snyder, 2012; Tisdell, 2012). Moreover, recent studies are selecting settings outside of formal education classrooms like workshops, retreats, travel, study abroad, and adult learners of English as a second language (Morgan, 2010; Ross, 1997; Taylor \& Snyder, 2012). However, although Mezirow's theory has matured enough to start using other methods, much can still be learned from traditional qualitative research like grounded theory data collection, ethnographic observation techniques, and phenomenological variations to advance understanding of this theory (Merriam \& Kim, 2012).

\section{Paradigmatic Underpinnings to TLT}

There are diverse theoretical perspectives that explain why humans transform including spiritual experiences (Dirkx, 2012), ecological awareness (O'Sullivan, 1999, 2012), marginalization (Johnson-Bailey, 2012), a transformative class/instructor (Ettling, 2012), life crises (Mezirow, 2000), awareness of societal power structures (Brookfield, 2012), and experiencing other cultures through travel/tourism/study abroad (Morgan, 2010). However, most of the literature surrounding transformative learning contains elements of three paradigmatic underpinnings: constructivism, humanism, and critical social theory (Cranton \& Taylor, 2012). Transformative learning theory uses a constructivist epistemology, meaning that much of how learning takes place within TLT is based on this constructivist view of knowledge: knowledge exists within people's brain and they interpret experiences by examining, questioning, and revising perceptions based 
on previous experiences. Next, a humanist axiology shows what values are important within TLT. Humanism posits that humans are inherently good, value personal freedom and autonomy, have a capacity to become self-actualized, and have a responsibility to make their own life better while also improving the conditions of those around them. Finally, a critical social ontology urges individual constructivist epistemology to be tied to political action, thereby creating structures, systems, and institutions that equalize access to healthcare, education, and economic social mobility (Brookfield, 2012). Therefore, TLT is at the intersection of personal and social, which hopefully leads to a greater sense of social accountability and action (Taylor \& Snyder, 2012).

Constructivist epistemology. The first paradigmatic underpinning of transformative learning is a constructivist epistemology. Epistemology deals with the nature of knowledge and how humans systematically arrive at knowing something (Falk, et al., 2012; Merriam \& Kim, 2012; Taylor \& Elias, 2012). Liable (2000) calls epistemology "a branch of philosophy that investigates the origin, nature, methods, and limits of human knowledge" (p. 686). Epistemology deals not with what we know, but how we know--our way of knowing (Kegan, 2000). Because transformative learning theory is a learning theory, it is essential to understand how theorists operating under this model believe learning is constructed.

Constructivism assumes that there exists no single, objective reality. Rather, in both constructivism and TLT, knowledge is what an individual creates, or constructs, in the mind versus what can be scientifically measured, or what others tell us is right or wrong (Fosnot \& Perry, 2005). Matching constructivism's independent construction of 
knowledge, Mezirow (2012) stated, "As there are no fixed truths or totally definitive knowledge, and because circumstances change, the human condition may be best understood as a continuous effort to negotiate contested meanings" (p.73). Furthermore, K. Taylor and Elias (2012) postulate that "we do not merely gain knowledge and experience as we mature (the informational explanation for change and growth); we also know in a different way (the transformational explanation)" (p. 151, italics in original). Moreover, a defining characteristic of transformative learning that matches constructivism maintains that individuals interpret personal experiences in their own way by examining, questioning, and revising perceptions based on previous experiences (Cranton \& Taylor, 2012). In this way, TLT studies the making or remaking of individual meaning (Dirkx, 2012). In a review of empirical studies on TLT from 20062010, E. W. Taylor and Snyder (2012) noted that a growing body of research suggests that "fostering transformative learning is not a 'one size fits all' approach, but rather it is necessary to consider the individual in a particular context" (p. 45).

Mezirow (2012) argued that all understanding is contextual—dependent on the circumstances, culture, and experiences of the individual. Likewise, Merriam and Kim (2012) claim that, in TLT, reality is how individuals interpret it to be in their minds, meaning there will be multiple perspectives, constructions, or interpretations of reality. Similarly, Falk, et al. (2012) posit that the outcomes of transformative learning are highly individual, that learning is a process of constructing meaning, that learning is dependent on context, and that learning is a cumulative process that happens over many experiences and interpretations of those experiences. Likewise, Taylor and Elias (2012) 
comment on the complexity of context: "at every moment, we seek to make sense of what goes on around us and within us; however, the process of that sense-making changes as it becomes more complex" (Taylor \& Elias, 2012, p. 151).

Knowing how you know something to be true also involves awareness of context (Mezirow, 2012). These contextual understandings are one of the reasons Mezirow defends the need for constructive discourse within TLT. He maintains that humans become aware of how we know what we know and the values that lead to our different perspectives by “trying on" (p. 86) another person's perspective.

Another nuanced version of constructivism in the transformation process is becoming aware of what we do not know we know. Cranton and E. W. Taylor (2012) assert that we uncritically assimilate perspectives from our social world, community, and culture. These perspectives go unchallenged until we find a situation that contradicts former experiences. Therefore, they maintain that transformative learning happens when an "alternative perspective calls into question a previously held, perhaps uncritically assimilated perspective" (Cranton \& Taylor, 2012, p. 8). In this way, we construct narratives or myths around that which we unconsciously construct of our lives, causing meaning-making to involve "unconscious, imaginative, and extrarational processes" (Dirkx, 2012, p. 116). Consequently, transformative learning analyzes, defines, and explains how outward expressions are products of unconscious mental constructs. Similarly, Dirkx (2012) proposed "consciousness always stands in relation to unconsciousness. Whereas consciousness refers to what we know, the unconscious constitutes what we do not consciously know" (p. 118). Thus, TLT helps us develop 
consciousness, or become aware of both what we display for the rest of the world and what we experience in our brains.

Constructivist scaffolding and transformative andragogy. Constructivist teachers are not solely teaching subject matter, giving tests, and transferring knowledge. Rather, they scaffold activities directed at developing the mind of active, rather than passive, learners (Harris \& Graham, 1994). Constructivists believe individuals learn through a series of experiences (Dewey, 1910). Both the concept of an active, engaged learner, and the idea of learning through experiences match TLT (Mezirow, 2000). Consequently, in both transformative learning theory and constructivism, education must engage and expand experience.

From a constructivist perspective, negative encounters of oppression, marginalization, and disorienting life crises might be seen as experiential learning rather than an outside person or system imposing external stimuli on an unwilling participant. Fenwick (2000) noted that almost all constructivists agree that a "learner is believed to construct, through reflection, a personal understanding of relevant structures of meaning derived from his or her action in the world" (p. 248). This inward mental searching closely matches the transformative learning theory view of critical reflection. In order to "think like an adult" (Mezirow, 2012, p. 73), one must learn to become aware of, and justify—or reframe — personal assumptions. This happens through critical reflection: "The research on transformative learning is ever growing, particularly as a means to frame pedagogy, with explicit practices for fostering critical reflection, self-efficacy, and an overall constructivist approach" (Taylor \& Snyder, 2012, p. 47). 
Piaget, a constructivist, saw learning in childhood and adolescence pass through a system of four stages. The final developmental stage, called the formal operational stage, deals with how adolescents learn to develop thought that moves them into adult ways of thinking (Piaget \& Inhelder, 2000). Learning, from Piagetian constructivism, requires abstract thought and problem-solving skills. Piaget's formal operational stage closely parallels Mezirow's (1998) theory on how critical reflection leads to reframing of underlying assumptions. Transformative learning theory maintains that as individuals move into adulthood, it becomes necessary to develop a more critical worldview in understanding their experiences (Taylor, 2008). Also, Mezirow (1997) declared that transformative learning should foster critically reflective thought through constructs of imaginative problem posing/solving; discourse that is learner-centered, participatory, interactive, and involves group deliberation and group problem solving; and small group discussion. Consequently, an educator scaffolds learning through metaphor analysis, group discussion, learning contracts, group projects, role play, case studies, concept mapping, life histories, participation in social actions, and classroom simulations of real life events, which are consistent with constructivism's use of scaffolding and focus on lived experience. Moreover, learners are frequently and repeatedly asked to identify the underlying assumptions behind their actions and beliefs as they participate in these activities (Brookfield, 1998; hooks, 1994). Accordingly, these methods encourage discourse and critical reflection, which should bring about a broader, more inclusive worldview (Mezirow, 1997, 1998). Students are encouraged to assess how they know something to be true and the consequences of actions based on such knowledge. 
According to TLT, students transform fixed frames of reference (or construct knowledge) by critically reflecting on assumptions in two different ways (Mezirow, 1998). One is by "objective reframing," which involves becoming critically reflective of a text, narrative, or premise and then analyzing and redefining the problem. Objective reframing is the most common form of transformative learning. Another type of transformative learning is "subjective reframing," which focuses on critical reflection on our own epistemological underpinnings and critical examination of the reasons for our limited frames of reference. Critical reflection requires students to understand the intentions, purposes, feelings, values, and moral decisions behind what someone means when they communicate an idea (Mezirow, 2000). Subjective reframing is much more difficult than objective reframing (Mezirow, 1998) because the disorienting dilemma it requires compels one to look inward rather than outward to see how one's values and beliefs lead to distorted, constrained, or discriminating ways of being (Brookfield, 1998, 2002). Therefore, subjective reframing may be too difficult or too painful for younger learners (Merriam, 2004). Neo-Piagetian constructivist scholars likewise argue that Piaget's age of adolescence might be too early for humans to fully acquire all of the tools for abstract thought (Case, 1992). This is one reason that TLT is considered an adult model of education.

Humanist axiology. Transformative learning theory's second paradigmatic underpinning is a humanist axiology (Brookfield, 2012; Pritchard, Morgan, \& Ateljevic, 2011). Axiology is the branch of philosophy that deals with what is intrinsically "good" or valued by a person or paradigm (Guba \& Lincoln, 2003). Humanism was one of the 
predominant theoretical paradigms in the 1970s when Mezirow's theory was developing, and TLT fits with this philosophy (Baumgartner, 2012). Humanism is based on the following underlying assumptions: (1) human nature is inherently good, (2) individuals are free and autonomous (capable of making major personal choices), (3) human potential for development is virtually unlimited, (4) how individuals view themselves plays a major role in development, (5) individuals desire self-actualization, (6) reality is personally defined, and (7) individuals have responsibility to improve both themselves and others (Cranton \& Taylor, 2012). Aligned with humanism, Mezirow (2012) stated that freedom, agency, tolerance, social justice, rationality, and adults' capacity for continual improvement provide essential norms for TLT. Mezirow's belief that a liberated person is free from unwarranted, unjustifiable beliefs or unsupportable attitudes is congruent with humanism. Furthermore, inclusion and egalitarianism are underlying values of both humanism (Bolman \& Deal, 2008) and the transformative learning model (O’Sullivan, 1999; Taylor, 2008). Finally, as individuals become increasingly autonomous and reflective, they move toward consciously constructing personal knowledge/assumptions and become increasingly responsible for their actions (which is how humanism works with constructivism mentioned above) (Taylor \& Elias, 2012). Maslow's hierarchy of needs had a strong influence on adult education, but on Mezirow in particular (Cranton \& Taylor, 2012). Maslow's self-actualization is deeply embedded within TLT. Mezirow (2012) stated that "hungry, homeless, desperate, threatened, sick, or frightened adults are less likely to be able to participate effectively in discourse to help us better understand the meaning of our own experiences" ( $\mathrm{p}$. 
82). Aligned with Maslow's hierarchy of needs, TLT assumes that humans share a need for connectedness, common interest, communication, and to realize learning potential (Mezirow, 2012). Additionally, a humanist axiology presupposes that individuals bring patterns of behavior - both positive and negative - learned in early life to educational settings (Brookfield, 1998). Thus, a humanist imperative is to establish how choices made from these patterns shape social contexts within the classroom (Bolman \& Deal, 2008). Moreover, core assumptions underlying individual behavior have strong influence on classroom actions, and are a key component of the transformative learning theory (Mezirow, 1998, 2000). Because personal relationships are a key part of humanism, understanding what a student brings into the classroom is necessary for transformative learning (Ettling, 2006).

Humanism in the transformative classroom. TLT's focus on lifelong learning shows the strong value it places on the humanist core assumption of almost limitless potential for human growth (Cranton \& Taylor, 2012). Falk, et al. (2012) identified four distinguishing features of adult lifelong learning within TLT: "the recognition of both informal and formal learning; the importance of self-motivated learning; an emphasis on self-funded learning; and the idea that participation in learning should be universal" ( $p$. 909). All of these characteristics ideally occur in a supportive environment, which also shows TLT's humanist underpinnings (Baumgartner, 2012). In order to give full support, an educator must be responsible for developing compassion, empathy, and awareness and have the unseen and the voiceless students always present with her or his thoughts, actions, worldview, and especially, in andragogical practices (Johnson-Bailey \& Alfred, 
2006). Therefore, educators must constantly work to become aware of personal assumptions, paradigmatic underpinnings, and worldview (Mezirow, 2000). Ettling (2006) stated:

What is needed is a series of guidelines that can be held in common by humans everywhere, regardless of race, culture, or societal status. Each of these viewpoints increasingly supports a consciousness on the part of the educator to recognize the dynamic nature of the learning situation and assume the responsibility inherent in a leadership role. (p.61)

Moreover, educators should not attempt to indoctrinate or coerce their students to make changes (Mezirow, 1997; 2012). Rather, they become collaborative learners with their students and encourage their students to do the same with each other (Mezirow, 2000). This vision of teacher and adult student on a similar developmental trajectory toward transformation highlights TLT's underlying value of humanism (Taylor \& Elias, 2012).

Critical social theory ontology. The third theoretical underpinning to transformative learning is critical social theory ontology. Ontology is the "branch of philosophy that studies the nature of existence" (Liable, 2000, p. 686) or what we believe about the nature of reality (Merriam \& Kim, 2012). Cranton and E. W. Taylor (2012) maintain that this particular theoretical underpinning may seem misplaced after reading about the theoretical underpinnings of constructivism and humanism. They state, "when we first come to critical social theory, at first glance there seems to be a serious disconnect with the previous philosophical perspectives" (Cranton \& Taylor, 2012, p. 8). 
While humanist axiology emphasizes empowerment of individuals, the critical social ontology is organized around power-who has it and who does not (Bolman \& Deal, 2008). Furthermore, interdependence, scarcity of resources, divergent interests, and power relations are realistic and everyday features of educational systems. From a critical social ontology, one comes to understand that governmental, legal, educational, and social systems have been historically designed to oppress, marginalize, and to reinforce white, male, heterosexist patriarchy (Brookfield, 2012). Consequently, discrimination and unfair, biased policies, norms, and practices exist within our educational organizations to an extent that freeing the entire system of inequity will be a slow process if current structural frames remain. Although the original intent of public education was to eliminate oppression, reliance upon educational institutions and organizations that created hundreds of years of systematized repression has not successfully eradicated problems of racism, sexism, heterosexism, ethnocentrism, and classism within schools (Marina \& Holmes, 2009; O’Sullivan, 1999). The futility lies in expecting the organizations and systems that created the problem to fix the problem (Bolman \& Deal, 2008).

Within TLT there exists a tension between individual awareness and societal change, however. The critical social theory stance goes beyond the constructivist goal of understanding a phenomenon and moves to empower participants to change their lives for the better. Instead, the critical social theory paradigm insists that participants act to improve society, as well. Transformative theorists posit that although reality is multiple (constructivist) it is also highly political, leading to contrasting dynamics of privilege and 
subjugation (Merriam \& Kim, 2012). In order to better understand oppression, critical research draws from critical race, feminist, and queer studies (Merriam \& Kim, 2012). Additionally, transformative educators help students understand that capitalist culture conspires against collaborative thinking and teaches individuals to think adversarially rather than collectively (Mezirow, 2000). Moreover, in order to critique and change society, critical social theory maintains that Western democracies are highly unequal societies where economic disparity, racism, and class discrimination exist, and the dominant meta-narrative perpetuates these inequities as normal, natural, and inevitable (Brookfield, 2012; Cranton \& Taylor, 2012).

The individual aspects of TLT (the epistemology) are only the beginning of transformation, however. A critical social theory perspective requires individual epistemology to be tied to political action: "to creating structures, systems, parties, and institutions that equalize access to common resources to democratize access" to healthcare, education, and economic social mobility (Brookfield, 2012, p. 136). Therefore, TLT lies at the intersection of personal and social, which should lead to a greater sense of social accountability (Taylor \& Snyder, 2012). Consequently, despite critiques to the contrary, TLT has both individual and social dimensions (Mezirow, 2000). Mezirow (2012) stated, "transformation theory attempts to explain this process and to examine its implications for action-oriented adult educators" (p. 74). Likewise, Brookfield (2012) maintains that the only way to change hegemonic forms of thought and practice is to change the structures and systems that produce and uphold dominant ideologies. 
Both TLT and critical social theory challenge dominant ideology, which is the broadly accepted set of values, beliefs, myths, explanations, and justifications that appears to be true and morally desirable to a the majority of the populace (Cranton \& Taylor, 2012). At the heart of TLT is the idea that we uncritically assimilate beliefs, assumptions, and perspectives as the natural and normal way to act-- even when they are in direct contrast to our own needs/interests (Brookfield, 2012; Mezirow, 2000). Thus, when we realize they are against our own needs/interests, a TLT experience opportunity emerges (Cranton \& Taylor, 2012). Additionally, Merriam and Kim (2012) maintain that "some realities are privileged over others and that for people to be empowered to free themselves from an oppressed state, the power dynamics of any situation must be uncovered and made visible" (p. 61). Furthermore, Mezirow (2004) stated that arriving at a more inclusive epistemology should transcend the restraints of ideological, political, religious, class, gender, and race constraints. Finally, according to transformative learning theory, in order to be able to understand presuppositions about race, gender, and sexual orientation, it is imperative that all humans recognize that we are all interconnected and connected to the earth (Ettling, 2006).

Mezirow's theoretical underpinnings also refer to Freire (Baumgartner, 2012; Mezirow, 2000) in that he sees similarities in TLT and Freire's concept of "conscientização" (Freire, 2000, p. 109). However, the differences between Freire's pedagogy of the oppressed and Mezirow's TLT are in epistemology, or how knowledge is constructed. Freire (2000) sees the village influencing transformation, whereas Mezirow (2000) states that transformative learning starts from an inward personal 
disorienting dilemma. Social constructivists, like Freire, see learning as an active social and cultural process whereby a series of multiple interpretations of differing worldviews leads to a shared sense of reality (Kezar, Carducci, \& Contreras-McGavin, 2006). In contrast with constructivism, social constructivists concentrate on how the context of social interactions influences the thoughts and actions of the individual rather than how the mind of the individual perceives reality devoid of social interactions (Freire, 2000). Consequently, constructivism (TLT) sees social interactions as how the mind perceives and responds to them (inward looking out), while social constructivism sees the lower level biological-mental functions being formed and developed by higher level sociocultural influences (outside forming in) (Matusov \& Hayes, 2000). Therefore, both Freire and Mezirow had goals of developing awareness and social action, but many scholars would argue that Mezirow's theory didn't go far enough toward social action (Brookfield, 2012; Cranton \& Taylor, 2012; Curry-Stevens, 2007; Johnson-Bailey, 2012; Johnson-Bailey \& Alfred, 2006; O’Sullivan, 1999, 2012; Tisdell, 2012). Likewise, Merriam and Kim (2012) assert, "For Freire, the goal of education is to become aware, through critical reflection and action (praxis), of the various oppressive forces in the world in order to transform it" (p. 66) (i.e. society permeating individual to collectively act). In contrast, Mezirow $(2000,2004,2012)$ insists that becoming aware of other viewpoints will eventually lead a person to social action (i.e. aware individual acting to improve society).

Critical social ontology in a transformative classroom. In order to uncover power dynamics that students bring with them into the TLT classroom, differences of 
culture, race, gender, sexual orientation, age, language, nationality and disability must be taught to students in a way that allows students to collectively work together for transformation (O’Sullivan, 1999). In transformative learning classrooms, students and teachers are taught to recognize individual and collective strengths (O'Sullivan, 1999). Transformative educators disrupt hegemony by unmasking power structures, overcoming alienation, teaching liberation, reclaiming reason, practicing democracy, and encouraging rational discourse (Cranton \& Taylor, 2012). Hegemony transpires when individuals "embrace (and see as normal) the conditions that serve those in power but work against the people's own interests" (Cranton \& Taylor, 2012, p. 9; see also, Brookfield, 2012). Similarly, Cordero and Rodriguez (2009) define hegemony as "the deliberate social, political and economic dominance of a particular group that saturates the consciousness of the nation" (p. 139). While critical exploration can help individuals understand their own pasts (Mezirow, 1997), “...critical theory’s focus on how adults learn to challenge dominant ideology, uncover power, and contest hegemony is crucial for scholars of transformative learning to consider if transformative learning is to avoid sliding into an unproblematized focus on the self" (Brookfield, 2012, pp. 131-132).

Transformative educators work to challenge systems of privilege and oppression to create emancipatory learning (Tisdell, 2012). Later qualitative studies of TLT have been less about describing TLT and the nature of it and more about fostering transformative learning (Merriam \& Kim, 2012). Indeed, Mezirow (2012) states: "fostering...liberating conditions for making more autonomous and informed choices and developing a sense of self-empowerment is the cardinal goal of adult education" (p. 
90). Therefore, a transformative educator realizes that transformative learning is emancipatory, and therefore critical in nature, because it aims to free educators from hegemonic power structures that exist between teacher and students and among students. Furthermore, Mezirow (2000) maintains that both teachers and students must realize that every belief or action benefits some while discriminating against others. Likewise, Ettling (2006) argues that transformative learning "implies a deep structural shift in one's consciousness and way of being in the world" and "presumes an authentic, value-based awareness" (p.65).

Educators must come to understand that all educational ideals have political implications (hooks, 1994; Mezirow, 2004). Likewise, Ettling (2006) states "issues of race, gender, class, and ethnicity are constituent elements of the learning situation and place ethical demands on the educational process and the educator" (p. 61). She also maintains that the position, perspective and power of the teacher are always present in the classroom, even if she or he is trying to be a moderator or facilitator of discussions. Therefore, differences of culture, race, gender, sexual orientation, age, language, nationality, and disability must be taught to students in a way that allows students to collectively work together for transformation (hooks, 1994; O’Sullivan, 1999).

\section{Constructivism, humanism, and critical social theory acting together.} Because ignorance of privilege is intrinsically systematized within our educational systems (hooks, 1994; McIntosh, 1990), individuals occupying positions of privilege and power will have to act first in order to break down existing barriers to equity thereby 
allowing transformation to come about in classrooms, educational organizations, and society (O'Sullivan, 1999). Therefore, change will be enacted only as individuals within educational systems are brought to more inclusive paradigms of understanding of others (Mezirow, 2000). Consequently, educators must guide students, other teachers, administrators, and legislators to break free of oppressive policies, practices, systems, and organizations if social change is to take place (O'Sullivan, 1999). Furthermore, transformation will take place only as the educators themselves are brought to more inclusive paradigms of understanding (Mezirow, 2000). From both a humanist axiology and a critical social ontology, it is evident that students are marginalized because of systemic inadequacies to identify racist, heterosexist, and patriarchal underpinnings that public schools have perpetuated (hooks, 1994). Ideally, as students become aware of their own assumptions, not only will they become more comfortable in educational settings, they will also be more productive and successful (Bolman \& Deal, 2008).

\section{Toward Political/Social Action: Critical Social TLT.}

Over almost 40 years, Mezirow's theory has generated much discussion, research, expansion, and critique (Baumgartner, 2012, Taylor \& Cranton, 2012). Nonetheless, the most repeated critique of TLT maintains that it is overly focused on individual transformation and does not deal directly with social transformation (Tisdell, 2012). Mezirow (2000) asserts that a person must realize that every belief or action benefits some while discriminating against others. However, he also maintains that arriving at a more inclusive epistemology should be independent from ideological, political, religious, class, gender, and race constraints (Mezirow, 2004). It is this 
political-social neutrality that recent scholars criticize (Brookfield, 2012; Johnson-Bailey, 2012). Many scholars argue that Mezirow's model of TLT is overly individualistic (Brookfield, 2012), gendered (English \& Irving, 2012), raced (Cranton \& Taylor, 2012; Johnson-Bailey \& Alfred, 2006), self-centered rather than earth-centered (O’Sullivan, 2012), and North American (or Western value)-centered (Ntseane, 2012).

E. W. Taylor and Snyder (2012) call for an engagement of theoretical frameworks beyond Mezirow's dominant perspective to help create allies for social justice: "It is fundamental to theoretical growth for researchers to draw on multiple conceptions, inclusive of critique, that will potentially lead to a more integral view of transformative learning theory" (p. 42). Additionally, Cranton and E. W. Taylor (2012) state: "despite the exponential growth of transformative learning theoretically, most research today continues to be based on Mezirow's work rather than the newer perspectives" ( $p$. 16). Throughout this paper, I will call this push for greater social action and transformation critical social transformative learning theory (critical social TLT). In order to push TLT toward social transformation, critical social TLT focuses on how North American ideology is saturated with "burgeoning inequalities in income and by pervasive power imbalances that are starkly illuminated through class, race, sexual orientation, gender, and ability" (Curry-Stevens, 2007, p. 33). The dominant North American narrative and ideology educates individuals to believe that capitalist society is organized in their best interest, when the opposite is often true (Solórzano, 1997). This cultural environment permeates powerful institutions, and therefore acts powerfully to maintain distorted personal perspectives (Taylor \& Elias, 2012). Brookfield (2012) 
maintains that if transformation entails a fundamental reordering of the most deep-rooted and paradigmatic assumptions that we hold, a student must be able to "discern how the ethic of capitalism and the logic of bureaucratic rationality push people into ways of living that perpetuate economic, racial, and gender oppression" (p. 134). Consequently, the goal of a critical social TLT educator is not merely personal transformation. Rather, educators who share this worldview help students take action to create a more fair society by understanding and then challenging the reproduction of social, political, and economic domination.

Critical social TLT educators must foster transformation that challenges students to identify dominant ideology, disrupt hegemony, unmask power, and then develop agency to oppose ideological forces that work against their best interests. However, Brookfield (2012) noted that purely individual changes are made possible only when structures, systems, and institutions change. Therefore, he argues that the developed world needs a societal disorienting dilemma in order to change culture, ideology, and reified forms of thought. In order to change capitalist culture, critical social educators utilize education, social protest, and social policy advocacy to narrow inequality (CurryStevens, 2007). Moreover, educators coming from a critical social TLT perspective focus on transformative education that inspires students to live more deeply rather than merely think more deeply (Tisdell, 2012).

In order to disrupt TLT's individual focus, critical social TLT asks, "Who is granted the opportunity to achieve autonomous thinking? Who is excluded, cast as the Other to be excluded and, by implication, dominated?" (Mezirow, 2012, p. 91). Obvious 
inequities in social structures influence how one understands experience, especially within classrooms. Moreover, many teachers-especially teachers from historically privileged groups - fail to notice how their own unfair and biased presuppositions about students and learning negatively affect the learning process (Johnson-Bailey \& Alfred, 2006). Likewise, hooks (1994) notes, "most of my professors were not the slightest bit interested in enlightenment. More than anything they seemed enthralled by the exercise of power and authority within their mini-kingdom, the classroom" (p. 17). Therefore, it is imperative that educators listen carefully to both what is voiced and unvoiced in the educational context. Consequently, critical social transformative learning theory recognizes those who have been silenced and allows educators to attend more fully to marginalized voices in the classroom (Johnson-Bailey \& Alfred, 2006).

Curry-Stevens (2007) calls Mezirow's focus on helping learners come to grips with their own power a "pedagogy of the privileged" and states that through TLT:

White learners can learn about racism, men can understand patriarchy, and ... both upper- and middle-class learners can understand neoliberalism and class exploitation. Unlike popular education, this form of pedagogy is not neutral. Rather, it is counterhegemonic in its goals and works within a framework of praxis, whereby assisting in transformation is linked with becoming an ally in struggles for justice. (p. 34)

It is important to note that educators, college students, travel participants, and workshop attendees are historically members of privileged, rather than oppressed groups. Therefore, this "pedagogy for the privileged" intentionally seeks to "engage 
privileged learners in workshops and classrooms and to assist in their transformation as allies in the struggle for social justice" (Curry-Stevens, 2007, p. 33).

Another well-cited critique of Mezirow's transformative learning theory comes from Johnson-Bailey and Alfred (2006), who argue that the model largely ignores and fails to fully include culturally-bound and consequently traditionally quiet classroom members (Tisdell, 2012, Cranton \& Taylor, 2012). Johnson-Bailey and Alfred, as members of marginalized groups, argue that marginalized members of the class often remain silent and, therefore, invisible from the class discussion. Consequently, the educator's responsibility, according to Johnson-Bailey and Alfred, is to ensure that marginalized class members have full access to and fully participate in class discussions (see also, hooks, 1994). Like Mezirow, they agree that full discussion is the key to transformative learning. They assert that "students bring a wealth of experience and knowledge from their varying histories, contexts, geographies, and biographies. It is [the teacher's] responsibility to recognize, highlight, and honor their contributions" (p.52). Johnson-Bailey and Alfred, therefore, conclude that educators need to allocate lesson time to specifically invite traditionally marginalized members of society to join the class discussion in ways that are not tokenizing or further underscore their already marginalized status.

\section{Critical Race Theory as a Framework for Understanding Critical Social TLT}

There needs to be a deliberate and conscious strategy to employ a political framework like activism or consciousness raising within TLT (Taylor \& Snyder, 2012). Critical and emancipatory research shows a critical stance that seeks to not only 
understand a person--rather it analyzes the power dynamics of a situation and critiques the status quo, and seeks to empower participants to transform. Therefore, critical race theory, feminism, critical ethnography, and participatory action research fall into this category (Merriam \& Kim, 2012). Likewise, Mezirow argues, "it is essential to seek out and encourage viewpoints that challenge prevailing norms of the dominant culture in matters of class, race, gender, technology, and environmental protection" (p. 79).

A critical research perspective is beginning to have a major presence in TLT (Merriam \& Kim, 2012). However, power relationships between instructor and students, and researcher and participants, must be examined in order to move to the critical social TLT discussed by recent scholars. Because transformative learning theory is not a political theory, it makes a poor framework for analyzing political systems, institutions, and organizations that impact transformation (Taylor \& Snyder, 2012). Therefore, another framework must be used to critique policies that affect educators' capacity to carry out transformative learning activities in a classroom. Furthermore, many TLT researchers assert that a race-centric perspective like Johnson-Bailey and Alfred call for can be effectively used in TLT (Cranton \& Taylor, 2012; Taylor \& Snyder, 2012; Tisdell, 2012).

What is CRT? Critical Race Theory (CRT) is a form of oppositional scholarship used to "uncover racial inequity and legal injustice" which "inform strategies of resistance" (Taylor, 2006, p. 72). Repeated themes in CRT scholarship include: exposure of racism as normal; understanding interest convergence; deconstruction of the myths of colorblindness within educational institutions; promotion of voices and narratives of 
people of color; and exposure of White, middle-class values as normative (Delgado, 2009a; Ladson-Billings, 1998; Litowitz, 2009; Smith \& Stovall, 2008; Taylor, 2006). Each of these concepts will be explained later in this section.

Intersections of themes between CRT and transformative learning include advocating for a critical pedagogy requiring students to assess power relationships and underlying assumptions behind educational ideals (hooks, 1994; O’ Sullivan, 1999). Furthermore, both transformative learning and CRT scholars argue that education should be emancipatory—freeing students from oppressive educational systems, while motivating students to access higher education, improve social mobility, and disrupt hegemonic power structures (Mezirow, 1997; Taylor, 2009). Moreover, both theories place a high priority on critical exploration to identify the social, economic, political, educational and psychological systems that contribute to underlying assumptions of individuals within educational systems (Mezirow, 2000; Steele, 2009).

CRT is a form of scholarly resistance that rejects traditional positivistic forms of research (Bell, 2009). Instead, CRT scholars argue that oppression is best understood from the experiences or vantage point of the oppressed (Leonardo, 2009). Rejecting the experiences of Whites as the standard, many CRT theorists use first-person storytelling, narrative, autobiography, science fiction, illustrations, anecdotes, allegories, humor, sarcasm, and parables as a way to expose/challenge/deconstruct racial social constructs (Bell, 2009; Litowitz, 2009; Taylor, 2009).

CRT is a sub-division, shares a history with, and most scholars would maintain is derived from Critical Legal Studies (CLS) (Taylor, 2009). CLS began in the 1970s when 
a growing number of legal scholars became concerned when civil rights legislation gains from the 1960s began to slow down or reverse. CRT in the law is a critique of CLS because CLS scholars failed to specifically address race and racism within the United States' legal framework (Parker \& Lynn, 2009). CRT in education came about as a result of similar concerns in the 1980s and 1990s when some scholars saw the achievement of people of color not being sufficiently addressed by mainstream academic scholars, legislators, and curriculum writers (Ladson-Billings, 1998).

A major critique of CRT is that there is not a unified theory that articulates exactly what CRT is and what it is trying to achieve (Litowitz, 2009). However, by reading articles from CRT scholars, several predominant themes are repeated often, and will be the focus of this section. Convergent CRT themes addressed in academic literature include:

- Racism is normal;

- Race is a social, not biological, factor that changes with the needs of dominant society;

- Interest convergence;

- Myths of color-blindness, post-racial society, and meritocracy; and

- Understanding Whiteness through the lens of White supremacy.

Racism is normal. The most-often repeated theme in CRT is that racism is normal. When CRT scholars use the term normal to describe racism, they are not indicating that racism is so prevalent that it is inevitable or insurmountable (Gillborn, 2009). Rather, they maintain that racism is neither aberrant, nor rare, but is deeply 
embedded within the framework of American society (Ladson-Billings, 1998; Parker \& Lynn, 2009; Taylor, 2009). Furthermore, racism is endemic, ingrained, and ordinary- a permanent, rather than marginal factor (Delgado \& Stefancic, 2001; Solórzano \& Yosso, 2009). Because CRT scholars recognize how much is still needed in overcoming racism, they seek to inform and advance the struggle for greater equity (Gillborn, 2009). CRT scholars contend that awareness of marginalization leads individuals to validation and affirmation, which, in turn, will hopefully lead to social action (Delgado-Gaitan, 1993; Ladson-Billings \& Donner, 2005; Smiley \& West, 2012)

Within CRT scholarship, racism is usually not defined as the acts of individuals working independently (for descriptions of individual acts of violence, however, see Solórzano, 1997; Sue, et al., 2007). Rather, racism describes embedded codes, customs, and conventions in governmental, institutional, and corporate systems that promote White supremacy (Taylor, 2009). Institutionalized White supremacy, or Whiteness, can take both active and passive — and conscious, and especially unconscious — roles (Tatum, 2009). In addition, within education, curricula, instructional techniques, assessment methods, school funding, and even desegregation reinforce the "White supremacist master script" (Ladson-Billings, 1998, p. 29; see also, Bell, 2009; Smith \& Stovall, 2008; Solórzano, 1997; Tushnet, 2009). Furthermore, Leonardo (2009) states that racism is so deeply embedded that even people of color add to or participate in their own oppression through reinforcing hegemony or "inter-minoritarian politics" (p. 268). Moreover, those who oppose racism are often marginalized regardless of racial privilege (Tatum, 2009, p. 286). 


\section{Race is a social, not biological, construct that changes with the needs of}

dominant society. In understanding racism, it is imperative to understand that race is a social, not biological construct, that changes with the needs of the dominant culture (Delgado \& Stefancic, 2001; Parker \& Lynn, 2009; Solórzano \& Yosso, 2009). In CRT scholarship, the term "Black" is used to describe marginalized societal social factors, while "White" is used to describe dominant culture (Ladson-Billings, 1998). Under this Black/White construct, Ladson-Billings (1998) provides an excellent example of how being a college professor can make her "White" in comparison to her Spanish-speaking, Latino gardener due to class dominance. It is important to note, however, that some CRT theorists challenge the use of these terms. For example, Solórzano and Yosso (2009) include African-Americans, Latinos, Asians, Pacific Islanders, and American Indians in the dialogue stating that it is important to shift the discussion of race from a White vs. Black narrative to a more complex discussion with many faces, voices, and experiences. Nevertheless, for the purposes of this paper, the terms of "Black" and "White" will be used in the way Ladson-Billings utilizes them because the terms align with the majority of critical race theorists. I also capitalize the terms "Black" and "White" to show societal racial categories of systematized oppression rather than feeding a biological myth of human races based on lightness and darkness of skin tone.

The marginalization of European Catholics in the 1800s provides an example of how the social construct of race changes with the needs of dominant society. As persecution in the United States intensified against Irish and Italian immigrants, Jesuit priests formed private schools to avoid the forced assimilation of Catholic children into 
the dominant protestant religious society (Slattery, 2013). Throughout the 20th Century, European Catholics moved into mainstream American culture and gained wealth and power, and became "White" (Delgado \& Stefancic, 2001). Meanwhile, Latina/o Catholics also moved to the United States and were forced out of the "White" category by census data (Ladson-Billings, 1998). As European Catholics assimilated to U.S. cultural and linguistic norms, it became harder to distinguish them from other "White" groups. However, Latina/o Catholics, went through the same process, but became a separate racial group.

Interest convergence. Building off of the concept that race changes with the needs of dominant culture is the concept of interest convergence. E. Taylor (2009) states, "The concept of interest convergence has its roots in the Marxist theory that the bourgeoisie will tolerate advances for the proletariat only if these advances benefit the bourgeoisie even more" (p. 5). Furthermore, interest convergence stems from the liberal agenda that presumes that social conditions for people of color will improve through slow and protracted reforms to the legal system (Parker \& Lynn, 2009). In a critique of slow moving liberal reforms, Delgado (2009a) maintains "liberals and moderates lie awake at night, asking how far they can take this affirmative action thing without sacrificing innocent white males" (p. 110).

Several examples of interest convergence will illustrate how liberal reforms benefit dominant groups over people of color. First, White women have been the primary beneficiaries of affirmative action legislation, receiving far more jobs and college opportunities than people of color (Ladson-Billings, 1998). Next, desegregation 
legislation has benefited White individuals even more than Black individuals evidenced by the data on how the achievement gap between underserved populations and White populations continues to grow (Bell, 2009). "Freedom of choice" schools, charter schools, magnet schools, private schools have made it so that despite 1954 Supreme Court case Brown v. Board of Education, "today most black children attend public schools that are both racially isolated and inferior" (Bell, Jr., 2009, p.74). Moreover, Dudziak (2009) states that the White interest in Brown v. Board of Education was not to represent people of color. Rather, as the United States fought wars in Southeast Asia against communism, it looked bad to the international community that people of color in our own country were treated like second-class citizens. Furthermore, Bell (2009) declares that Civil Rights Act of 1964 had as many illusions as gains: "the interest of blacks in achieving racial equality will be accommodated only when it converges with the interests of whites" (Bell, Jr., 2009, p. 76).

Another example of interest convergence is educational reform. Although almost all of the educational reforms contain a stated imperative to increase marginalized students' educational outcomes, Black students are further behind their White classmates than they were in the 1980s (Gillborn, 2009). E. Taylor (2006) posits that, from a CRT framework, the term "achievement gap" does not adequately address disparities in how students of color are currently underserved in educational institutions. Therefore, E. Taylor (2006) proposes that academics create studies to understand why schools are underserving students of color. Additionally, Gillborn (2009) points to data that indicate that Black students score, on average, 15 points lower than White students on IQ 
tests. This is evidence of both interest convergence and race as a social, rather than a biological construct. Because there is no cognitive genetic difference between White students and students of color, CRT scholars argue that the IQ tests serve as proof that Blacks have been underserved in educational settings rather than to show some form of cognitive or cultural deficit (Bell, Jr., 2009). However, when strategies fail to achieve desired results it is the student of color, not the strategy, who is found to be lacking (Ladson-Billings, 1998). Furthermore, Black students are more under-represented in gifted and talented programs and over-represented in special needs programs than they were when the major reforms started in the 1980s (Gillborn, 2009). Additionally, normative assumptions about achievement, policy, schooling, and research; limited and simplistic policy language; micro political aggressions; and underrepresented narratives of marginalized individuals within textbooks have further increased the achievement gap between White students and students of color (Marshall \& Gerstl-Pepin, 2005).

Finally, the most disheartening example of interest convergence is how educational institutions are serving as a gateway for institutionalizing students of color toward prison in many parts of the country. Stovall (2013b) stated that schools in poor, Black and Latina/o neighborhoods in Chicago now have police officers and booking stations within public schools. Subsequently, this and similar policies have resulted in more African American adults being under correctional control today than were enslaved in 1850 (Smiley \& West, 2012).

Myths of color-blindness, post-racial society, and the meritocracy. Hegemony is a value that goes along with the idea of interest convergence. Hegemony is oppression 
that does not occur by physical force. Rather, power in hegemonic structures occurs when the dominant culture exercises influence over subordinate parts of society to get the subordinate parts of society to become complicit in their own oppression. In CRT, hegemony is achieved through myths of color-blindness, post-racial society, and meritocracy (Delgado \& Stefancic, 2001; Parker \& Lynn, 2009; Stovall, 2013a). These comforting myths (for manyWhites) do not express the real struggles, conflicts, and experiences of people of color because they provide a normalized, mono-cultural view of modern institutions (Gillborn, 2009). Furthermore, CRT scholars are suspicious of broadly accepted paradigms of objectivity, methods of empirical data collection, and claims of colorblindness within educational and legal scholarship (Taylor, 2009). Consequently, one of the purposes of CRT research is to challenge the dominant ideologies of color-blindness, post-racial society, and the meritocracy (Leonardo, 2009). Myth of colorblindness. Colorblindness is the idea that our legal and educational systems treat all students equally regardless of what racial group to which they belong (Litowitz, 2009). The myth of colorblindness secures oppression through ahistorical justifications, white-washing of history, and "sleights of mind" (Leonardo, 2009, p. 265). One example of an historical "sleight of mind" is that students are led to believe they have no part in re-creating racism and that United States history has been a slow march toward equality. Likewise, sanitizing of history reimagines civil rights history as a rational, linear, incremental march toward equity (Gillborn, 2009). Therefore, Leonardo (2009) calls for teachers and students to unpack racism through open discussion to alleviate "racial amnesia" and color-blind discourses (p. 268). 
The No Child Left Behind Act of 2001 (NCLB) serves as an example of how supposedly colorblind statutes negatively impact people of color. E. Taylor (2006) argues that the sanctions implemented for failure to meet adequate yearly progress in NCLB "triggers a series of escalating and increasingly intrusive sanctions, and has generated fear of sanctions, especially in predominantly minority schools" (p. 71). He asserts that racial inequalities in testing are normal (universal), occurring in cities, suburbs, and rural areas. Because there are no biological mental differences between White students and students of color, E. Taylor contends that the testing data prove that US public education underserves poor and minority students. He further maintains that the achievement gap is double at graduation than what it was when students enter kindergarten, further proof that public school are failing students of color. Thus, E. Taylor (2006) posits, from a CRT framework, the achievement gap has not been treated as a "racial achievement gap" (p. 72), stating that the reasons for disparity between White and minority students is undertheorized and underconceptualized (see also Stovall, 2013). He critiques the normative assumptions of colorblindness as a major flaw in NCLB. Rather than punishing struggling students and schools by cutting federal funding to them as outlined in NCLB, E. Taylor calls for studies and funding to understand why schools are underserving students of color.

Myth of a post-racial society. The myth of the post-racial society is the idea that we have come so far in civil-rights laws and educational reforms that we have "done enough" (Tushnet, 2009) so that race is no longer an issue in the United States. Tatum (2009) addresses this dominant narrative: "Basically I grew up thinking racism was no 
longer an issue" (p. 280). While most Whites maintain that things are better for Blacks today, few of them would want to trade societal places with African Americans (LadsonBillings, 1998). Likewise, Smiley and West (2012) quote Robert F. Kennedy, "If any man claims the Negro should be content...let him say he would willingly change the color of his skin and go to live in the Negro section of a large city. Then and only then has he a right to such a claim" (p. 59).

Another claim to a post-racial society is anti-essentialism, which is to deny that race or gender exists, so there is no need to produce, study, or understand the social constructs of those marginalized groups. Crenshaw (2009) counters: "But to say that a category such as race or gender is socially constructed is not to say that category has no significance in our world" (p. 244).

High stakes testing required by educational reforms of the 1990s and 2000s serve as an example of how the myth of a post-racial society negatively impacts all students. From a combined transformative learning and CRT perspective, high-stakes tests like the type required by NCLB are also detrimental to authentic learning outcomes of White, upper- and middle-class students. While marginalized students are forced to confront their lack of privilege daily as a means of survival (Johnson-Bailey \& Alfred, 2006; O’Sullivan, 1999), standardized tests reinforce stereotypes that essentialize poor and minority students as "underachieving" rather than "underserved" (Taylor, 2006). These stereotypes can create a false sense of achievement among White learners and educators thereby hindering academic growth because they are already passing tests that were designed by individuals and for individuals from their own culture. Furthermore, 
educators and students from privileged groups often struggle to see the inherent flaws in their perceptions of others (Ettling, 2006). Therefore, Brookfield (1998) argues that it becomes almost impossible for White teachers and students to see the flaws in their own assumptions.

Myth of the meritocracy. The American Dream-the belief that you can achieve and acquire anything if you work hard enough — is what undergirds the myth of the meritocracy (McNamee \& Miller, 2004). Many Americans are confident they deserve what the have — power, authority, status, and/or wealth—-because they have received it due to their own talent and effort (Liu, 2011). Therefore, meritocracy is the myth that if one works hard enough and manages finances just right, that anyone can achieve social mobility in the United States (Tatum, 2009). Interestingly, the more unearned privileges in society such as race, gender, or social class an individual has, the more they seem to adhere to the "pull yourself up by your bootstraps" philosophy (McIntosh, 1990). However, these individuals do not understand that the United States political and legal structures provide unearned financial and social benefits to individuals of White European heritage (Taylor, 2009).

Understanding Whiteness. The myths of colorblindness, post-racial society, and meritocracy mask the dominance those who are constructed as White have over people of color in US society. White privilege is maintained through the "psychological and material benefits from an unearned position in society" (Leonardo, 2009, p. 273). Moreover, the omni-presence of White Supremacy makes it difficult for privileged 
classes to see political, legal, financial, and social benefits of being White (Gillborn, 2009).

It is important to note that there is a difference between being a White person and the political construction of Whiteness (hooks, 1994). Whiteness encompasses the acts, decisions, policies, laws, systems, and institutions that allow racial hegemony to saturate every day life for people of color (Leonardo, 2009). Additionally, the United States political and legal structure is based on overt and embedded codes established by property owners (middle and upper-class Whites), while Blacks were constructed as property, making African Americans property transformed into citizens (Ladson-Billings, 1998). Consequently, humanity in historical texts about the United States constitution is meant as White, male, and propertied because "equality" fails to address slavery, patriarchy, and industrial capitalism woven into United States' original documents (Leonardo, 2009). Throughout United States history, those construed as White have secured "property" rights, or unearned privileges through their socially constructed Whiteness (Bell, 2009; McIntosh, 1990). Indeed, Leonardo (2009) argues that while the process of securing this domination was complicated and multi-faceted, Whites "set up a system that benefits the group, mystifies the system, removes the agents of actions from discourse, and when interrogated about it, stifles the discussion with inane comments about the 'reality' of the charges being made" (Leonardo, 2009, p. 272).

Because racism is deeply embedded within our laws and codes of conduct "people do not generally see the ways in which they are privileged, and so well-intentioned, middle-class, white liberals often strive to maintain privilege for their children, while 
denying they are doing so" (Brah \& Phoenix, 2009, p. 253). Furthermore, educational media and textbooks "conjure up images of domination happening behind the backs of whites, rather than on the backs of people of color," creating a system of domination removed from agents of oppression (Leonardo, 2009, p. 262).

\section{TLT Process}

The purpose of the following section is to explain the transformative learning process and define key terms relating to that process. However, it is important to note that the transformative learning process does not occur in a systematic series of steps that must be completed in order. Rather, different elements combine to create conditions where an individual is open to changing her or his worldview. Throughout this section I will explain how cognitive dissonance and disorienting dillemmas lead an individual to objective and subject reframing of an individual's frame of reference. Over time, a series of these events can lead to a reordering of the way an individual sees the worlds and acts in it.

Humans acquire a working knowledge of informal logic, beliefs, natural dispositions and skills for reflection through life experiences, parents, culture, and education before arriving at adulthood (Mezirow, 1998). Usually this system of beliefs, called a frame of reference, is represented in intentions, morals, feelings, and assumptions that may or may not be completely understood by the individual (Kegan, 2000). According to Brookfield (2012), this system of beliefs, assumptions, perspectives, and worldview govern a person's thoughts in a particular situation. Cognitive dissonance and disorienting dilemmas are situations that take us by surprise and cause us to question 
assumptions based on our frame of reference (Mezirow, 2000). Transformation occurs when the disorienting dilemma then leads an individual to expanded awareness, critical reflection, validating discourse, and reflective action as one moves toward a greater sense of autonomy and agency.

Frame of reference. In order to understand how people transform in TLT, it is imperative to understand what form they are transforming (Kegan, 2000). Mezirow (2012) maintains that we all come to derive meaning from philosophic, economic, social, and psychological systems that take place in our everyday lives. This meaning perspective is the "structure of assumptions and expectations through which we filter sense impressions" (Mezirow, 2012, p. 82). Meaning perspectives comprise various meaning schemes: expectations governing perceived cause/effect relationships, roles, values that connect feelings, experiences, judgments, and actions in various situations (Baumgartner, 2012). Whether we intentionally or unintentionally incorporate these meaning schemes, they become part of how we see the world and our place in the world. These "socialized ways of knowing" (Taylor \& Elias, 2012, p. 152) become what is called a "frame of reference" (Mezirow, 1997, 2000). A frame of reference consists of the mental structures of thoughts and feelings - shaped by social, economic, political, religious, and psychological structures — that help us understand ourselves and our relationships (Baumgartner, 2012). Collectively held frames of reference result from the way we interpret experiences and represent cultural paradigms as a community.

A frame of reference consists of two major subsets: a habit of mind and a point of view. A habit of mind is a set of broad, generalized, orienting predispositions that filter 
our interpretations of experiences (Mezirow, 1997, 2000). A point of view incorporates clusters of expectations, beliefs, feelings, attitudes, and judgments that shape our judgments, causalities, stereotypes, expectations, and our idealized self-image. Both habits of mind and points of view tend to exist outside of our awareness (Mezirow, 2012). Frames of reference, however, encompass values, sense of self, and give us a sense of stability, coherence, community, and identity. More dependable frames of references are "more inclusive, differentiating, permeable (open to other viewpoints), critically reflective of assumptions, emotionally capable of change, and integrative of experience" (Mezirow, 2012, p. 84). Similar to Mezirow, Kegan (2000) maintains that a frame of reference is a way of knowing that encompasses rules, values, ideals, prejudices, passions, promises, betrayals, personal demons, and terrors (Kegan, 2000).

Mezirow (2000) also explains that humans transform our frames of reference through critical reflection on the assumptions upon which our interpretations and beliefs are based. He argues that understanding the meaning of personal experience opens the possibility for reflective thought, intellectual development, personal transformations and enlightened social change. Consequently, based on critical reflection, Humans can reframe self-concepts and goals, as well as the criteria for evaluating self-concepts and goals (Baumgartner, 2012).

In addition to the frame of reference, many TLT scholars use the word "worldview" to describe how one sees the world and acts in it. For example, O'Sullivan (2012) uses the term "worldview," calling transformation a "profound change in worldview" (p. 164). To O'Sullivan a worldview encompasses beliefs, assumptions, 
attitudes, values, and ideas to form a comprehensive model of reality. Because the definitions and the way the authors talk about frame of reference and worldview are so similar, I use the terms worldview and frame of reference interchangeably throughout this dissertation. Additionally, the term "meaning perspective" and frame of reference became synonymous for Mezirow by the early 2000s, meaning: "the structure of assumptions and expectations through which we filter sense impressions" (Mezirow, 2000 , p. 17). However, the term "meaning perspective" has changed meanings several times over the course of 40 years of Mezirow's writings, so it is nearly impossible to get a comprehensive definition from the various writings (Baumgartner, 2012). Therefore, unless specifically referring to Mezirow's writings, I do not use the term "meaning perspective" to avoid confusion for readers of this paper.

Cognitive dissonance. Frames of reference that may have worked for us as children often do not as adults (Mezirow, 2012). Cognitive Dissonance occurs when a person is confronted with an idea or experience that contradicts a prior epistemological assumption (Ettling, 2006; Mezirow, 1998, 2000). Subsequently, cognitive dissonance can serve as a catalyst for critical reflection (Ettling, 2006).

Cognitive dissonance occurs when an individual challenges previously unquestioned assumptions about oneself (Taylor \& Elias, 2012); realizes that a life stance, or way of being in the world is distorted, incomplete, or intolerant (Willis, 2012); and begins to scrutinize personal beliefs (Johnson-Bailey, 2012). Reframing of this magnitude can lead to a complete reordering of epistemological assumptions through personal, intentional, inward soul searching (Mezirow, 2000). It typically takes a series 
of cognitive dissonance experiences, accompanied by critical reflection, to bring about a change in a frame of reference leading to a paradigmatic shift (hooks, 1994; Taylor, 2008). However, because it is difficult for individuals to shift worldviews (hooks, 1994), cognitive dissonance is often accompanied by feelings of fear, guilt, shame, resistance, and sadness, even in willing participants (Bennetts, 2003).

In much of Mezirow's early work he studied factors that encouraged or discouraged adult women to re-enter college after an extended period of time away from school (Baumgartner, 2012). One of the most common underlying assumptions that impacted their worldview was the belief that "a woman's place is in the home" (Mezirow, 2012, p. 86). If a woman with that frame of reference meets another woman she believes is a good mother, and finds out that the other mother works out of the home, she is confronted with cognitive dissonance (i.e. how can the woman be a good mother and work outside of the home?). In an article explaining cognitive dissonance, K. Taylor (2008) gives another example of how cognitive dissonance might occur: a woman's "race places her in a dominant group; because she rarely experiences dissonance related to her race, she does not actively explore what it means to be White until she begins working closely with a group of young women of color" (p. 226). From these examples, it is evident that if a worldview goes unchallenged (either by ideas or experiences), cognitive dissonance will never occur. Many scholars maintain that individuals in positions of privilege are the least likely to experience cognitive dissonance because of their socioeconomic ability to associate with people of similar mindsets and privileges (hooks, 1994; Johnson-Bailey, 2012; O’Sullivan, 2012). Consequently, individuals in positions 
of privilege might make an excellent candidates for transformative learning (CurryStevens, 2007).

Ideally, cognitive dissonance serves as a catalyst for critical reflection, and later, transformation (Taylor, 2008). In order to guide a person to understand that they are experiencing cognitive dissonance, it is often beneficial when a supportive teacher or friend can explain what is happening (Ettling, 2006). hooks (1994) notes: "It is difficult for individuals to shift paradigms and there must be a setting for folks to voice fears, to talk about what they are doing, how they are doing it, and why" (p. 38). Unlike disorienting dilemmas (discussed below), cognitive dissonance does not necessarily compel one to see the world differently. Indeed, K. Taylor (2008) notes: "Although a sense of dissonance signals the individual to look for a new way of making meaning, it may lead to regression if too extreme or if unmediated by supportive others or positive personal attributes" (p. 230). She also maintains that if individuals fail to experience cognitive dissonance at all, then no transformation will happen, leading to a stall in cognitive, intrapersonal, and interpersonal growth.

Much of the TLT literature uses the terms cognitive dissonance and disorienting dilemma synonymously. However, I separate the terms because I believe that the kind of reframing they require leads to different types of transformative learning. While some transformations alter our very being, our core, our sense of self, and how we define ourselves to the world, there are also incremental transformations that happen often--even daily--that transform our frames of reference (Tisdell, 2012). In my experience as an educator, these incremental transformations are often the results of dozens or hundreds of 
encounters with cognitive dissonance (see also, Ettling, 2006). As articulated by K. Taylor (2008) above, cognitive dissonance can be rejected if the resulting disorienting dilemma does not attend the cognitive dissonance. Likewise, Mezirow (2012) explained that "transformations in habit of mind may be ... incremental, involving a progressive series of transformations in related points of view that culminate in a transformation in habit of mind" (Mezirow, 2012, p. 86). Furthermore, while cognitive dissonance may or may not lead to transformation, a disorienting dilemma usually leads to transformation. Therefore, because the kind of transformation resulting from the cognitive dissonance and a disorienting dilemma are different, I will not use them interchangeably even though it is often the case in TLT literature.

Disorienting dilemma. As a person works through one (or more) experience(s) that challenge a previous paradigm, inner conflict leads to a "disorienting dilemma," which requires a complete reordering of epistemological assumptions (Mezirow, 2000, p. 22). A disorienting dilemma characteristically results from a major life transition, change in roles or relationships, acute personal or social crisis, death of a loved one, an intercultural experience, or some other major life change (Ettling, 2006; Taylor, 2008). In transformative learning theory, however, a disorienting dilemma is not necessarily a grand scale phenomenon; rather, transformation takes personal, intentional, inward soul searching to achieve true transformation (Mezirow, 2000). What separates a disorienting dilemma from cognitive dissonance is that the affective emotions accompanying a disorienting dilemma are too great to reject, ignore, or dismiss (O’Sullivan, 2012). 
Although a disorienting dilemma typically involves a major life change, the process can also be brought about by an educator (Ettling, 2006). According to Ettling, educators can create classroom activities and conditions that help students understand the underlying assumptions to their beliefs. However, educators must be aware that altering a student's basic assumptions can be met with fear and resistance even when the student is willing to go through the transformative learning process. Because of the sensitive nature of bringing about disorienting dilemmas, educators must realize that transformation requires attention, time, integrity, and trust (Brookfield, 1998, 2002; Ettling, 2006). Mezirow (2000) indicated that willingness from both teachers and students was a key component to initiating a disorienting dilemma. Additionally, K. Taylor and Elias (2012) caution that although intense, disorienting dilemmas are not always negative. They add: "such experiences illuminate and challenge heretofore invisible and unquestioned assumptions that determine how we know ourselves and the world around us" (p. 150).

Because disorienting dilemmas are often attended by intense emotions, they often lead to epochal transformations. Epochal transformations are dramatic changes that challenge an individual's core identity, worldview, or very sense of who they are (Mezirow, 2012). Epochal transformations invite a person to live more deeply, which cultivates hope in the capacity for personal growth. In addition, emancipation from unsustainable attitudes and behaviors often encourages an individual to make society a better place for others (Tisdell, 2012). Once a person has had an epochal change they will never go back to the old way of seeing the world (Mezirow, 2012; O'Sullivan 1999, 
2012). While it is possible for an individual to experience cognitive dissonance without transformation of assumptions, it is much more difficult to experience a disorienting dilemma without leading to transformation of a person's frame of reference (O'Sullivan, 2012).

Full literacy is necessary in TLT. Mezirow (1996) stated that becoming fully literate is necessary for transformative learning and critical reflection to take place. He believed that literacy involves two essential processes, infrequently recognized by educators: participating constructively in rational discourse and learning to identify and critically analyze one's own and others' assumptions. Mezirow further stated that memorizing meanings and acquiring basic skills are insufficient. Under a TLT paradigm, curricula, instruction, and assessment must emphasize critical reflection on assumptions and discourse. In addition, skills involved in becoming a fully literate learner include the capacity to interpret, judge, solve problems, learn how to learn, and learn to become increasingly self-directed as a learner. All of these skills foster critically reflective thought and participation in discourse.

Merriam (2004) argues that some of these skills are beyond students in K-12 learning environments. Mezirow $(2004,2012)$ concurs that the reason TLT is an adult learning process is because epistemic cognition emerges in late adolescence (although its form changes throughout adult years).

Reflective discourse. According to Mezirow (1997), communicative learningdiscussion between two or more people trying to understand the meaning or justification behind beliefs - is necessary to bring about transformative learning. Therefore, learning 
in TLT requires an individual to use language to articulate experiences to oneself or others (Mezirow, 2012). In this communicative style of learning, one seeks to understand what others mean when they communicate by analyzing meaning behind words, becoming critically reflective of personal and others' assumptions, and increasing awareness of the intent or subtext of another person's dialogue. Likewise, Mezirow (2012) stated, "In communicative learning, we determine the justification of a problematic belief or understanding through rational discourse to arrive at a tentative best judgment” (p. 78). Moreover, reflective discourse occurs through consensus as we discuss our knowledge/beliefs with others (Baumgartner, 2012). In addition, through reflective discourse individuals develop cultural competence, which is an ability to negotiate personal purposes, values, feelings, and meanings rather than relying on others' beliefs. Discourse also encourages examining alternate perspectives, critical assessment of assumptions, and finding one's own voice. Throughout Mezirow's writings this kind of learning has been called rational discourse, reflective discourse, communicative learning, and dialogic learning (Baumgartner, 2012). For simplicity, I refer to this kind of learning simply as reflective discourse.

Mezirow (2000) argued that understanding the meaning of our experience opens the possibility for reflective thought, intellectual development, personal transformations, and enlightened social change. Commenting on the principles necessary to bring about communicative learning, which in theory should lead to transformations of thought, Mezirow (1996) stated: 
Ideally, participants in discourse would have full information about the issue and be: (a) open to alternative perspectives; (b) able to make justified inferences, analyze and reason argumentatively; (c) able to reflect critically on assumptions and premises; (d) aware of and manage one's emotions and be empathic to others; and (e) granted full equality as participants in discourse and freedom from coercion and distorting self-deception. ( $(\mathbb{4 0})$

Furthermore, reflective discourse involves awareness of relationships and power, empathy for others, and self-control. However, Mezirow (2012) cautions "our culture conspires against collaborative thinking and the development of social competence by conditioning us to think adversarially" (p. 79). In Western cultures argument, dualism, and partisanship are norms leading an individual to believe that there are only two sides to every issue (Mezirow, 2000). Consequently, the resulting increase in polarization leads to "the gradual extinction of thoughtful discourse" (Taylor \& Cranton, 2012, p. 158). Therefore, individuals in the United States (especially ones in positions of privilege) are growing more rigid and defensive rather than more permeable, which greatly hinders a person's capacity for transformation. Thus, an individual sets out to win an argument rather than seek mutual understanding. However, the quality of information is compromised in a dualistic culture because the information is less informed and dependable than those based on wider range of experiences (Slattery, 2013). In this polarized, dualistic environment, we have failed to recognize what we "other" and what part of "other" lives within ourselves (Taylor \& Cranton, 2012). 
Brookfield (2012) states that as individuals are taught to participate in reflective discourse, they must be taught to understand that some discourse oppresses individuals within society. To Brookfield, an individual should seek out a wide range of views and encourage dissention, but provisionally suspend judgment of the issue until all beliefs are stated, thereby exhibiting open-mindedness and a willingness to hear multiple perspectives. However, it is also human nature to protect oneself from topics that are delicate, sensitive, or contrary to an individual's frame of reference (Mezirow, 1998). Notwithstanding, a desire to protect oneself from delicate subjects silences opposing voices which reifies dominant society worldviews. Furthermore, educators must simultaneously use extreme caution not to romanticize, exotify, tokenize, superficialize, or essentialize already marginalized students' points of view (Gambrell \& Bright, in press; Morgan, 2010). Therefore, educators must be especially sensitive when teaching students to participate in reflective discourse (Ettling, 2006; Johnson-Bailey \& Alfred, 2008).

Another way that individuals in positions of privilege use silence to reinforce their privilege by refusing to voice concerns with discriminatory policies or practices. To avoid silence as a vehicle for reifying privilege, Mezirow (1998) includes a long list of organizational (or classroom) norms that inhibit critically reflective thought, and therefore, reflective discourse: avoiding sensitive discussions; interpersonal conflict about sensitive topics, or enforcing taboos about sensitive topics; keeping personal problems private; refusing to express organizational problems, injustices, or discriminating practices; making premature value judgments; making a decision and then 
keeping it private or avoiding public dialogue that might refute one's point of view; avoiding seeing how problems are interconnected; and tolerating offensive behavior in the educational setting (Mezirow, 1998). In order to create discourse that is free of these negative norms, a discussion with full information would be free from coercion, grant all members equal access to speaking/listening, give time for and expect critical reflection of personal paradigms, be open and empathetic to other beliefs, and search for common ground among the members' epistemologies (Mezirow, 1997). Therefore, teachers and students must be willing to listen, open to change, and ready to critically analyze personal thoughts before true discussion can take place (Mezirow, 1996).

Educators must understand that altering a student's basic assumptions can be met with fear and resistance even when the student is willing to go through the transformative learning process (Ettling, 2006). Furthermore, because of the sensitive nature of bringing about disorienting dilemmas, educators must realize that transformation requires attention, time, integrity, and trust (Brookfield, 1998, 2002; Ettling, 2006). Teachers must also be aware that disorienting dilemmas require a major reordering of students' expectations, norms and methodologies, and therefore be patient with students as they go throughout this process (Ettling, 2006). Likewise, transformative educators understand that the transformation process extends beyond the semester and may continue for years after students leave a class (Tisdell, 2012).

In order to create an appropriate environment for transformative learning to take place, K. Taylor and Elias (2012) maintain that learning environments must have both high support and high challenge. First, they develop nurturing, caring, trusting, and 
secure learning environments. Subsequently, they slowly begin to introduce activities that lead to disorienting dilemmas, thereby providing opportunities for discourse and reflection hoping this leads their students to new frames of reference or perspectives. Similarly, hooks (1994) states that it is important to scaffold activities to encourage willingness to listen, speak, and change for the right atmosphere to exist for a cooperative learning classroom. In a TLT classroom, teachers encourage students to actively listen, paraphrase each other, and be permeable to each other's thoughts and feelings (Mezirow, 2000).

Critique of Mezirow's emphasis on rational discourse. Lave (1996) questions if a teacher (in the traditional sense of the word) is even necessary for transformative learning to take place and maintains that the traditional classroom structure even serves to hinder traditionally marginalized students. Whereas a transformative, constructivist educator might be seen as an instructional coach rather than a knowledge dispenser, under Lave's model teachers and students are collaborative co-learners. By having students celebrate their diverse backgrounds and underlying assumptions, xenophobia, racism, sexism, and homophobia should diminish as students seek to understand each other. Furthermore, K. Taylor and Elias (2012) assert:

As we construct more complex identities and values, differences formerly experienced as uncomfortable or threatening can become instead a welcome source of new possibilities and perspectives. Rather than avoiding conflict for fear of damaging relationships, we find that "difficult dialogues" improve mutual understanding. (p. 153) 
Brookfield (2012) also states that there is an over-emphasis on the teacher's role, but goes a step further and questions if discourse is even necessary. He argues that much of TLT can be learned through life experience. O'Sullivan (2012) echoes this assumption: "There is no question that challenging discussion can stimulate change, particularly when there is safety, trust, and respect... however, there are other routes to transformative learning...We also understand that crucial learning often takes place nonverbally, in the inarticulate dimensions of our bodies" (p. 172). Johnson-Bailey and Alfred (2006) further state that the traditional classroom environment serves to silence historically disenfranchised students rather than to include them in the conversation. Thus, the teacher's power and position serves as a type of hegemony which must be constantly reduced by the educator (hooks, 1994). Therefore, marginalization and experience may be the best starting points for critical reflection (Johnson-Bailey, 2012).

Critical reflection. While there are multiple critiques of Mezirow's focus on reflective discourse, most TLT theorists maintain that critical reflection is necessary to bring about transformation (Brookfield, 2002, 2012; Ettling, 2006; Merriam, 2004; Mezirow, 1998). Critical reflection is a mental deliberation process whereby individuals come to understand not only their own pasts, but also social, economic, political, educational, and psychological power systems (Mezirow, 1997). Moreover, critical reflection requires students to understand intentions, purposes, feelings, values, and moral decisions behind what someone means when they communicate an idea (Mezirow, 2000). 
While marginalized students are confronted daily with inconsistencies between American social ideals and lived experiences (hooks, 1994; Johnson-Bailey \& Alfred, 2006), persons in positions of privilege or power have difficult cognitive and emotional work to break free of prejudiced ways of seeing the world (McIntosh, 1990; O'Sullivan, 1999). In order to break prejudicial worldviews, Mezirow (2012) maintains that individuals must realize that "understandings and beliefs are more dependable when they produce interpretations and opinions that are more justifiable or true than would those predicated on other understandings or beliefs" (p. 74). Therefore, it is essential for individuals to become critically aware of their tacit assumptions (and those of others) to assess the relevance of the underpinnings to an idea or statement. Furthermore, Mezirow (2012) stated, "informed decisions require not only awareness of the source and context of our knowledge, values, and feelings but also critical reflection on the validity of their assumptions or premises" (p. 76). Therefore, imagining alternative perspectives is strongly encouraged in TLT. Alternative perspectives come to light by learning new frames of reference, elaborating new frames of reference, transforming habits of mind, or by "trying on" another's point of view (Baumgartner, 2012). Mezirow (2012) argued, "We change our point of view by trying on another's point of view... The most personally significant and emotionally exacting transformations involve a critique of previously unexamined premises regarding oneself' (p. 86). Critical reflection occurs when an individual becomes aware of personal perceptions, meanings, behaviors, or customs whether they are affective, critical, conceptual, or theoretical in nature (Mezirow, 2012). 
Within a transformative classroom, the educator is seen as a facilitator and not as an authority figure, and progressively transfers leadership to the classroom (hooks, 1994). Consequently, students become increasingly adept at learning from each other. Furthermore, transformative learning is not a supplement or an add-on to a teaching style, but is central to the educator's methodology. Indeed, Mezirow (1997) asserted, "Critical reflection, awareness of frames of reference, and participation in discourse become significant elements in defining learning needs, setting educational objectives, designing materials and methods, and in evaluating learner growth using nonmaterial methods such as portfolios" (p.12).

Ideally, critical reflection of beliefs or ideology leads a person to emancipatory action, making the person whose worldview has transformed aware of the possibility to be an agent for change (Baumgartner, 2012; Merriam \& Kim, 2012). Likewise, Mezirow (2012) argued, "Taking action on reflective insights often involves overcoming situational, emotional, and informational constraints that may require new learning experiences in order to move forward" (p. 87). This newfound agency and autonomy are the intended result of the critical social TLT classroom.

Is the focus on critical reflection based on privilege? Recent empirical research concludes that reflective discourse and critical reflection may not be as effective in transforming frames of reference as experiences are (Baumgartner, 2012). Furthermore, several TLT theorists state that Mezirow's focus on reflective discourse and critical reflection needs to be situated in the context of his privilege as a White, male professor teaching mostly privileged teacher candidates (Curry-Stevens, 2007; English \& Irving, 
2012; Johnson-Bailey \& Alfred, 2006; Taylor, 2007). Moreover, E. W. Taylor and Snyder (2012) add that relatively "few studies explore whether there was something unique about the participants' background, culture, and/or positionality and their experiences in relationship to transformative learning” (p. 40). In addition, JohnsonBailey and Alfred (2006) maintain that marginalization may be the most powerful transformer. Similarly, E. W. Taylor (2007) argues that studies have shown that context may be the most important variable on transformative learning outcomes and calls for experimental approaches with different sociocultural variables (race, class, gender, and culture). However, because individuals occupy multiple contexts simultaneously, it will be difficult to ascertain the role of the influence of context on transformation within individuals (Baumgartner, 2012). However, despite the difficulty of identifying the role of contextual variables, Baumgartner maintains that studies like this need to be carried out.

\section{Critical Reflection Occurs as Individuals Assess Underlying Assumptions Through Both Subjective and Objective Reframing.}

Students transform fixed frames of reference by critically reflecting on assumptions in two different ways (Mezirow, 1998). One is by "objective reframing," which involves becoming critically reflective of another person's assumptions in a text, narrative, or premise and then analyzing and redefining the problem (Mezirow, 2012). Objective reframing is the most common form of transformative learning. Another type of transformative learning is "subjective reframing," which focuses on critical reflection one's own assumptions and critical examination of the reasons for limited personal 
frames of reference. Brookfield (1998) identifies the difficulty of subjective reframing: "becoming aware of our assumptions is a puzzling and contradictory task" (p. 197). He argues that it is almost impossible to see the flaws in personal assumptions and likened it to a "dog trying to catch its tail, or of trying to see the back of your head while looking in the bathroom mirror" (p. 197). Critical reflection requires students to understand the intentions, purposes, feelings, values, and moral decisions behind what someone means when they communicate an idea (Mezirow, 2000).

Objective reframing. Objective reframing involves becoming critically reflective of a text, narrative, or premise, and then analyzing and redefining the problem (Mezirow, 1998). Another term for objective reframing is critical examination (O' Sullivan, 2012). For example, when reading a text, an educator might ask, have students reflect upon, or write responses to the following questions: what are the underlying assumptions, values, beliefs, or intentions behind this text (Brookfield, 1998)? Whose voices are privileged/marginalized (hooks, 1994)? How do your life experiences reinforce or disagree with the text (Johnson-Bailey \& Alfred, 2006)? Objective reframing is the most common form of transformative learning because it typically takes less emotional work to identify untenable assumptions in the narratives of someone else (Mezirow, 1998). 
TRAVEL FOR TRANSFORMATION

Subjective reframing. Subjective reframing focuses on critical reflection on one's own assumptions and critical examination of the reasons for limited personal frames of reference (Mezirow, 1998). Subjective reframing can be about narratives, a system, an organization or workplace, feelings, or ways one learns (Mezirow, 2012). Subjective reframing requires one to look inward rather than outward to see how one's values and beliefs lead to distorted, constrained, or discriminating ways of being (Brookfield, 1998, 2002). Therefore, subjective reframing is much more difficult than objective reframing (Mezirow, 1998), and may be too difficult or too painful for younger learners (Merriam, 2004).

Another way of looking at objective/subjective reframing. K. Taylor and Elias (2012) describe another way to look at subjective reframing. It is important to note that their article is permeated by the developmental psychology of Kegan (2000). According to K. Taylor and Elias (2012), a person is subject to that which is "larger" than her or him. To be larger than an individual, the thing must be more complex, bigger than that person's ability to understand or change the item. Likewise, things that are "smaller" than a person are object to that person. To be smaller, the item or idea must be less complicated, meaning the individual can look at it, take responsibility for it, reflect upon it, exercise control or manipulate it, and change it to a new way of knowing. That which is subjective to us, we are run by, identified with, fused with, at the effect of. "We 'have' object; we 'are' subject”' (Taylor \& Elias, 2012, p. 151).

According to K. Taylor and Elias (2012), there are things we know we know (our recognition makes it object to us), there are things we don't know we know (its 
invisibility makes it subject to us), and then there are things we know we don't know (are object to us because we can change that). We are subject to that which we don't know we "know" (unjustified assumptions). Because we simply "know it," it is in charge, it makes us object and we are subject to it. This causes an assumption that we are "righter" than those who are not like us. We can simply ignore those that we perceive as "other." Therefore, K. Taylor and Elias (2012) maintain that this unjustified subjectivity makes a poor way of knowing for a society that grows more diverse daily.

Transformation. Although discussed all throughout this paper, the end goal of transformative learning theory is perspective transformation, the emancipatory process of becoming critically aware of how and why personal assumptions have come to restrain how we see ourselves and our relationships and then reframing assumptions to permit a more inclusive and discriminating integration of experience and then acting on the new perspective (Baumgartner, 2012). Mezirow (2012) states, “transformative learning refers to transforming a problematic frame of reference to make it more dependable in our adult life by generating opinions and interpretations that are more justified. We become critically reflective of those beliefs that become problematic" (p. 85). This change in worldview involves understanding the cognitive (conscious thoughts), conative (impulse to act), and affective (feelings about) dimensions of one's own frame of reference (Baumgartner 2012). Some transformations alter our very being, our core, our sense of self and how we define ourselves to the world. There are also smaller transformations that happen often--even daily--that transform our frames of reference over time (transpose consciousness, but not core identity). In conjunction with other efforts, these 
small changes can create core transformative change over time (Tisdell, 2012). Through the transformation of enough individuals, society can be changed as well by reformulating reified structures, reconstructing dominant narratives, and helping others become critically reflective of assumptions (Mezirow, 2012). Once transformation to a more open/permeable worldview happens, it is impossible to revert to the old perspective (Baumgartner, 2012).

\section{TLT and Travel}

One burgeoning area of critical social TLT literature is its intersection with travel, volunteer tourism, and study-abroad programs. Travel uniquely opens a person to transformative learning because it takes an individual out of daily routines and positions the learner into a state of "otherness," both geographically and culturally (Morgan, 2010; Ross, 2010). This otherness allows the traveler to experience cognitive dissonance and disorienting dilemmas described earlier. Additionally, travel, especially for volunteerism (Coghlan \& Gooch, 2011), spiritual or secular pilgrimage (Morgan, 2010), second language learning (Foster, 1997), or study abroad (Brown, 2009), carries with it the purpose of the traveler wanting to be changed (transformed) by the experience. This willingness to be changed by the travel experience creates a condition with increased odds of transformative learning (Mezirow, 2000). To be considered travel for transformation, the travel experience must respect the values and knowledge of the host culture, acknowledge the presence of differences in privilege, and utilize environmentally sustainable practices (Ross, 2010). About the power of transformative learning within travel experiences, Mezirow (1997) stated: 
An example of a habit of mind is ethnocentrism, the predisposition to regard others outside one's own group as inferior.... We can have an experience in another culture that results in our critically reflecting on our misconceptions of this particular group. The result may be a change in point of view toward the group involved. As a result, we may become more tolerant or more accepting of members of that group. If this happens over and over again with a number of different groups, it can lead to a transformation by accretion in our governing habit of mind. (pp. $5 \& 7$ )

Travel can be transformative, but is under-researched. Until very recently, few studies have explored the connections between travel and transformation, and even fewer have studied the elements of travel that lead to transformation (Ross, 2010). Moreover, Ross (2010) concludes that travel may be the best activity to lead one toward transformation of frames of reference, because individuals often undergo travel as a means of expanding consciousness (see also, Morgan, 2010). Furthermore, Ross (2010) posits that an individual usually wants something from travel that does not fit that person's paradigms, assumptions, or worldview. She states: "transformative travel and transformative tourism aim to honor the delicate interplay between the self and anyone who is different, or the 'other,' during travel" (p. 55).

Falk, Ballantine, Packer, and Benckendorff (2012) provide a review of both empirical and theoretical articles about tourism and transformation. According to Falk, Ballantine, Packer, and Benckendorff (2012), “tourism's concentrated, 'first- person' engagement with the culturally unfamiliar lends its subjects a mantle of cosmopolite 
authority that years of classroom instruction rarely approach"' (p. 909). However, they assert that the connection to travel and transformation is a relatively under-researched, under-theorized, and under-scrutinized field. In addition, they maintain that in addition to acquiring knowledge, individuals often look to tourism as a vehicle for changing themselves, their vision of themselves, and their vision of the world around them. They argue that learning that occurs in a travel situation almost always exceeds what could be learned about other cultures through formal classroom activities because individuals may construct a personal connection with people from another culture. Therefore, Falk, et al. (2012) believe that "the travel experience can contribute personal benefits to the individual visitor, to society, and the planet; benefits that long outlive the temporal boundaries of the experience itself" (p. 922).

Learning another language accelerates travel for transformation. In addition to traveling to a different culture, learning another language is often transformative (Foster, 1997; Goulah, 2007). However, it is an avenue toward transformative learning that is under-researched and remains largely theoretical (Goulah, 2007). Foster (1997) states that when learning another language, one has to learn not only vocabulary, grammar, syntax, and other communication skills, but how another culture thinks. Moreover, the learner becomes one with a new "culture, music, literature, film-the dynamics involved can often be quite destabilizing” (p. 35).

Although there are various reasons why students enroll in a foreign language course, there is typically a mild degree of anxiety or vulnerability associated with learning another language. According to Foster (1997), this vulnerability sets up an 
excellent situation for transformative learning to occur. Furthermore, she argues that learning to communicate in a different language is unsettling, causing a distorted view of self. Subsequently, the distorted self-perception leads students to feel trapped, but as communication skills develop, the language learners start to feel more liberated (Goulah, 2007).

Foster (1997) asserts that learning a second language can be linked to the destabilizing experience of Mezirow's (2000) disorienting dilemma: "This process can be a profoundly unsettling psychological proposition. The immediate interaction with the language and culture can directly threaten an individual's self-concept and worldview" (p. 35). Trying to participate in a second language involves taking risk. This risk requires the learner to relinquish her or his view as a competent communicator in the native language. As an example, Foster (1997) explains that if a person does not understand statistics, at least the individual is capable of clearly articulating the difficulty of understanding the material in a coherent manner. Contrarily, with learning a second language, the learner does not even possess the vocabulary or skills to express confusion, which, according to Foster, leads to a disoriented view of self. Thus, a key to second language acquisition, according to Ross (2010), is the learner's willingness to "to be like representative members of the 'other' language community, and to become associated, at least vicariously, with that other community” (p. 37). In addition, Goulah (2007) found that students who learned another language while immersed in that culture developed critical socio-cultural attitudes about their home culture. 
TRAVEL FOR TRANSFORMATION

To be transformative, travel must be for a purpose. Willingness to be changed by the travel experience increases when there is a purpose behind the travel whether it be volunteer tourism, study abroad, or a secular pilgrimage (Morgan, 2010).

Volunteer tourism. Coghlan and Gooch (2011) review literature at the intersection of TLT and volunteer tourism. They state that in the mid-2000s there started to appear literature that combined the idea of transformation and travel. Their article discusses volunteer tourism, which is a type of tourism where the traveler goes to another place specifically to do some form of service that benefits the host culture. According to Coghlan and Gooch (2011), elements that make volunteer tourism a perfect fit for transformative learning studies are the following: the individual seeks deeper involvement with the social and natural world, the change in context situates the learner in a position where cognitive dissonance and disorienting dilemmas are more likely to occur, and the desire of the tourist to attain self-actualization increases the traveler's capacity for transformative learning. Volunteer tourism increases the likelihood of a shift in underlying assumptions and a switch from the rational (cognitive) toward the affective (emotional/spiritual) dimensions of learning.

According to Coghlan and Gooch (2011), by stepping away from "the learner's socio-cultural context and the dominant ideologies" (p. 9), the traveler is able to develop the skills of critical reflection. Through critical reflection, the volunteer tourist first has a chance to learn about another culture (objective reframing), but then this learning can often be applied to the participant's culture of origin (subjective reframing). Therefore, there is an increased capacity for the traveler to situate herself or himself within the larger 
political, economic, and socio-cultural domain. Finally, volunteer tourism often allows the traveler develop awareness of social justice. This awareness opens the volunteer tourist to "re-appraising personal values and redressing power imbalances, [which] may be related to the issues of individuation, authenticity and emancipatory learning discussed in the literature on transformative learning" (p. 11).

Coghlan and Gooch (2011) note that many volunteer tourists experience "reverse culture shock" upon returning to their culture of origin and that they "may not be able to reconcile their skills, values and attitudes they developed during their volunteer tourism experience" (p. 12). Therefore, because the volunteer tourism has undergone transformation, attention must be paid to help orient the volunteer tourist back to the culture of origin when the travel experience is over.

Secular pilgrimage. Morgan's (2010) work focused on travel experiences of individuals that experienced transformation during travel abroad: "this article argues that by undertaking an actual journey involving a profound engagement with unfamiliar places and experiences, a person may experience a degree of disruption to their subjective orientation to the world (worldview or inner consciousness) sufficient to engender transformative learning" (p. 249). He situates the idea of travel for transformation within Mezirow's TLT concept of cognitive dissonance and disorienting dilemma leading to critical reflection and eventually perspective transformation. Morgan (2010) argues that the traditional classroom environment is not a sufficient vehicle for transformation because it does not situate the learner in a position of otherness. Travel, on the other hand, requires that the learner be engaged in a disruptive encounter with otherness that 
provides significant contrasts to ordinary home experience. "Otherness," According to Morgan, in the new culture can be identified with different cultural customs, mores, values, and attitudes. Typically, such experiences are likely when traveling to another country although a contrasting locality within the same country (e.g., urban dweller visiting a rural area or vice versa) could also lead to transformation of worldview.

For transformation to occur, the more "other" (both geographically and culturally) the place is, the more likely transformation will take place (Morgan, 2010; Ross, 2010). This feeling of being an "outsider" or "othered" that an individual might feel in a new environment increases the likeliness that a disorienting dilemma (often referred to as “culture shock") will occur (Mezirow, 2012; Morgan, 2010). Even more important, Morgan (2010) posits that participants who have experienced otherness are less likely to universalize, essentialize, or generalize other cultures within the home environment upon return. Furthermore, he asserts that transformation is most likely to occur if the traveler has the opportunity to reflect upon the experience either individually or collectively in order to reevaluate one's perspective of the other.

Morgan (2010) labels travel for transformation a pilgrimage, but says it is not with the obvious religious connections, but travel for personal development. Rather, he maintains a pilgrimage should "seek to elicit deep, experiential encounters with 'Otherness' through nature and wilderness vis-a'-vis through intercultural dialogue represents a particularly important direction for future research" (p. 263). However, Morgan warned:

Crucially, overemphasizing cultural Otherness over commonality runs the risk of 
exoticizing, romanticizing, essentializing, and superficializing the lived experience of people encountered through travel which is more likely to reify than transform existing frames of mind and consequent power asymmetries (Said, 2003), the very antithesis of transformative education. Travel has the potential to act as a powerful vehicle for transformative education. However, it is incumbent on all who wish to utilize such an approach to do so in an informed and ethically responsible manner. p. 264

Therefore, it is essential that the educator be prepared to guide students toward the subjective reframing domains of critical reflection so the focus becomes on one's own culture and self rather than over-emphasizing the "otherness" of the host culture.

Study abroad. Study-abroad programs are another medium through which travel has potential for transformative learning. Brown (2009) conducted an ethnographic qualitative research study that combined participant interviews and long-term observations of 150 post-graduate student participants from Asia, Africa, Europe, and Middle East in a yearlong study-abroad program in England. Brown (2009) questioned if exposure to a new culture had potential for transformation, increased tolerance, and made it possible to bridge the various cultural elements in their home countries leading to the development of a less ethnocentric perspective. She examined if prolonged absence from the student' home culture could help students re-vision or reframe their professional and domestic roles. Brown (2009) found that the duration, purpose of travel, and degree of immersion plays a significant role in perspective transformation. 
Removal from home environment for an extended period of time allowed students to experience freedom from cultural and familial expectations. Consequently, this increased freedom allocated space for students to experience self-discovery and transformation. Every student $(N=150)$ experienced transformation of cultural perspectives that would outlast their study-abroad and would carry implications in their future business and interpersonal roles. Almost all of the students experienced disorientation at early stages followed by increased autonomy, self-confidence, selfefficacy, vision of one's place in society, and self-control. In the final interview, most of the students viewed the sojourn as a life-changing, vision-altering, and irrevocable event. They all experienced concern at how their family, friends, and business associates would view their transformed state. Furthermore, some of the students became so adept at the new culture that many of them were apprehensive at re-entry to their culture of origin.

Travel for transformation: Pedagogy of the privileged. It is often privileged learners who can take part in travel for transformation because the traveler would either have to have the funds or access to institutional funds in order to afford travelling to an “other" place to experience study abroad. However, Curry-Stevens (2007) posits that a "pedagogy for the privileged, which seeks to transform those with more advantages into allies of those with fewer, presents a considerable impetus for broad, societal change" ( $p$. 35). She asserts that privileged individuals, if enlightened could be powerful allies for social change (see also, Bolman-Deal, 2008). Therefore, Curry-Stevens (2007) asserts it is incumbent upon adult educators to become more effective to motivate this group toward broader social justice objectives. She further contends that transformative 
learning theorists rarely distinguish between pedagogy of the oppressed and pedagogy of the privileged, making this area of study both under-theorized and under-studied.

In order to analyze and understand the pedagogy for the privileged, Curry-Stevens (2007) used grounded theory to analyze life history interviews of 20 educators to determine how their personal narratives of teaching privileged learners about oppression informed their teaching. She found that educators reported their students going through first a series of confidence shaking processes of awareness of oppression, awareness of oppression as structural, awareness as oneself as oppressed, locating oneself as privileged, understanding the benefits that flow from privilege, and understanding oneself as implicated in the oppression of others and understanding oneself as an oppressor. Following the disorienting processes, the educators found that students proceed to build confidence as an ally for social justice through building confidence to take action, planning what actions one will undertake, building confidence and agency focuses on arranging for ongoing support, and individuals in the group covenant with each other about their new commitments and plans to act for social justice. Curry-Stevens (2007) concluded, "Accordingly, pedagogy for the privileged, if successfully navigated, enables us to reconnect to all humanity—not just to those like us" (p. 40).

Critical social TLT and travel. Travel can also be a vehicle for critical social awareness and transformation. Pritchard, Morgan, and Ateljevic (2011) maintain that a method should be developed that combines transformative learning and social action to offer a distinctive approach to tourism knowledge production. They incorporate critical theory in trying to analyze transformative learning within tourism studies, closely 
aligning to what I labeled critical social TLT earlier in this paper. Like Curry-Stevens (2007), they claim that the dominant meta-narrative of the Western world's value system is at a crisis point environmentally, financially and politically. Pritchard, Morgan, and Ateljevic (2011) assert that the current use of travel as a vehicle solely for knowledge production is in need of change. Therefore, they state that a critical social TLT perspective is needed in tourism and travel abroad studies. They propose that travel and tourism offer time for reflection of one's values and one's place in the world that formal classroom studies do not. However, it is important to note that they are not merely arguing that tourism can allow time for critical reflection and activism. Rather, they posit that tourism and travel should be specifically designed to be transformative and give space for the tourist to participate in social action. According to the authors, this lack of specific directional time for transformative action and social action is a theory gap in the existing literature in both TLT and tourism studies.

In short, Pritchard, Morgan, and Ateljevic (2011) match the growing number of TLT scholars who challenge Mezirow's ontological assumption that transforming the individual is enough. Like the CRT and critical social theory critics of Mezirow, they maintain that social action should be a direct part of the travel experience. Concordant with this idea of purposeful social action the authors state: "And yet the continued conceptual development of tourism depends on the exploration of new paradigms and perspectives, because when we push ourselves away from dominant and taken-forgranted thinking we open up possibilities of seeing ourselves and our multiple worlds anew" (p. 943). This paradigm shift should be transformative, not only in how the 
individual thinks about other cultures, but how the individual acts toward and helps others act toward the host culture. Therefore, Pritchard, Morgan, and Ateljevic (2011) maintain that by being immersed in another culture, the traveler gets to experience a narrative separate from the dominant western narrative and that the individual can come to "value "planetary rather than national interests, eco-sustainability rather than sentimental environmentalism, feminism rather than heroic models, personal growth rather than personal ambition" (p. 944). Furthermore, they posit that tourism's complex, variegated, and interdisciplinary nature make it a perfect vehicle for critical social TLT work. Another benefit of tourism and study abroad, according to the authors, is that it opens a space to talk about race, class, gender, globalization, and community both in the host culture and in the place of origin.

Cordero and Rodriguez (2009) also maintain that travel should be created to be a medium for critical social TLT. They conducted a study wherein social work graduate students first took class on diversity and social justice education followed by a 12-day immersion experience in Puerto Rico to conduct social work. Cordero and Rodriguez (2009) argue that educators must prepare students in a culturally competent manner to prepare practitioners to understand and address the causes, dynamics, and consequences of oppression, thereby preparing practitioners to promote social justice. Furthermore, they maintain that cross-cultural learning and exposure is best accomplished through immersion experiences. They state "while most practitioners have taken a multicultural education course, fewer have immersion experiences where they could gain culturally specific practice experience with ethnic minorities and be supervised by instructors with 
such expertise" (p. 138). Through the course and the immersion experience, Cordero and Rodriguez (2009) found that participants experienced a more full appreciation and understanding of their own ethnocultural identity, increasing the participants' selfawareness and critical consciousness. Consequently, the increased awareness of other cultures lead students to desire social action for the marginalized. To be transformative, Cordero and Rodriguez (2009) assert that the transformative learning experience should engage students in a cross-cultural learning process in which they "examine, question and expand their cultural assumptions, acquiring behavioral and cognitive repertoires that foster critical consciousness" (p. 136). They suggest that a "multi-method, cross-cultural teaching approach can be used across ethnocultural groups and with diverse student groups to move beyond traditional classroom learning to include immersion learning from and within the community under study" (p. 147).

Travel/TLT conclusions. Although study abroad, volunteer tourism, and secular pilgrimages can be transformative, not every person who travels to another place experiences transformation. Therefore, it is important to unpack what elements of travel increase the odds of critical social transformation. Morgan's (2010) review of literature of empirical studies found the following criteria increased an individual's capacity for critical social transformation:

- Occurs in environments and contexts associated with a powerful, strong and positive "spirit of place," or "sense of place,"

- Provides experiential learning opportunities that are directly focused on encountering the otherness of nature and wilderness, 
- Provides experiential learning opportunities focused on encountering the otherness of, and dialogue with, other cultures,

- Permits interactions with fellow travelers and the host community through mutual learning and dialogue,

- Provides opportunities for solo experiences that allow space for reflection and contemplation, and

- Provides opportunities for meaningful environmental or social service work.

Ross (2010) also summarized factors that increased opportunities for transformation and positive behavioral change resulting from travel:

- A destination that pulls the individual as far as possible from known experiences (novelty),

- Intimate intercultural experiences involving in-depth discussions,

- Activities that stimulate contemplation resulting in meaning-making of the traveler's experiences, and

- Post-travel activities that help the sojourner to continue to reflect upon and extract meaning from the travel (p. 56).

In conclusion, in order to be considered travel for transformation, the travel experience must respect the values and knowledge of the host culture, acknowledge the presence of differences in privilege, and utilize environmentally sustainable practices (Ross, 2010). In addition, the duration, purpose of travel, and degree of immersion plays a significant role in perspective transformation (Brown, 2009). A reason that transformative study abroad is better positioned toward cognitive dissonance and 
disorienting dilemmas than the traditional classroom environment is that it situates the student in a new context where the place, culture, people, and hopefully the language are "other" (Morgan, 2010). Duration of time in "other" culture also has an impact: Cordero and Rodriguez' (2009) study with 12 days had limitations (not every student experienced perspective transformation), whereas every student in the Brown (2009) study where participants spent a year immersed in the host culture experienced irreversible transformation in their frames of reference. Moreover, willingness to be changed by the travel experience increases when there is a purpose behind the travel whether it is volunteer tourism, study abroad, or a secular pilgrimage (Morgan, 2010). In addition, learning another language can be disorienting because the second language learner has to learn not only vocabulary, grammar, syntax, and other communication skills, but how another culture thinks, (Foster, 1997; Goulah, 2007; Ross, 2010). It is also important that the transformative travel educator understands that study-abroad research is a "pedagogy of the privileged" wherein students are coming to understand their role as members of dominant society from an oppressive culture. Most importantly, the educator must motivate this group toward broader social justice objectives (Curry-Stevens, 2007). Finally, it would be ideal if a class about equity and social justice were combined with the study-abroad experience (Cordero \& Rodriguez, 2009).

\section{Literature Review Summary}

Transformative learning theory provides a framework for effecting change in a personal frame of reference leading to a more inclusive, permeable, and reflective epistemology (Ettling, 2006; Johnson-Bailey \& Alfred, 2006; Merriam, 2004; Mezirow, 
1996, 1997, 2000, 2004). Mezirow (2000) explained that we transform our frames of reference through critical reflection on the assumptions upon which our interpretations, beliefs, habits of mind, or points of view are based. Transformation commences with cognitive dissonance, when a person is confronted with an idea or experience that contradicts a prior underlying assumption of a personal belief system (Ettling, 2006; Mezirow, 1998, 2000). Cognitive dissonance then serves as a catalyst for critical reflection (Brookfield, 1998, 2000; Ettling, 2006), which in turn leads to a "disorienting dilemma," requiring a reordering of epistemological assumptions and causing a change in beliefs and behaviors (Mezirow, 2000, p.22).

Throughout the past decade, TLT researchers are looking beyond Mezirow for other theoretical orientations of transformative learning (Taylor \& Snyder, 2012). For example, Johnson-Bailey and Alfred (2006; see also, Johnson-Bailey, 2012) posit that marginalization may be the most powerful transformer. E. W. Taylor (2007) states that studies have shown that context may be the most important variable on transformative learning outcomes and calls for experimental approaches with different sociocultural variables (race, class, gender, and culture). E. W. Taylor and Snyder (2012) found that "few studies explore whether there was something unique about the participants' background, culture, and/or positionality and the experiences in relationship to transformative learning" (p. 40). Therefore, it is imperative to investigate what role participants' backgrounds have in transformative learning, especially what role race and culture play in cognitive dissonance/disorienting dilemmas. 
An existing theoretical framework for analyzing how race and culture affect transformative learning is critical race theory. Critical Race Theory is a form of oppositional scholarship used to "uncover racial inequity and legal injustice" which “inform strategies of resistance” (Taylor, 2006, p. 72, see also, Bell, 2009). Repeated themes in CRT scholarship include: deconstruction of the myths of colorblindness within educational institutions; promotion of voices and narratives of people of color; interest convergence; exposure of racism as normal—meaning both universal and embedded within statutes and policies; and exposure of White, middle-class values as normative (Delgado, 2009a; Ladson-Billings, 1998; Litowitz, 2009; Smith \& Stovall, 2008; Taylor, 2006). The myths of colorblindness, post-racial society, and meritocracy mask the dominance those who are constructed as White have over people of color in United States society. White privilege is maintained through the "psychological and material benefits from an unearned position in society" (Leonardo, 2009, p. 273). The omni-presence of White supremacy makes it difficult for privileged classes to see political, legal, financial, and social benefits of being White (Gillborn, 2009). Because it is a well established theory regarding race and other forms of systemic oppression, CRT is well-suited as a framework for analyzing and giving vocabulary to how White students transform into more tolerant ways of seeing the world and acting in it (Bright \& Gambrell, 2013).

Recent studies (2009-2012) have additionally explored the role that settings outside of formal education classrooms like workshops, retreats, and adult learners of English as a second language play in transformative learning (Taylor \& Snyder, 2012, p. 29). Additionally, a growing body of research explores the effects of travel, tourism, and 
study-abroad programs on transformation of cultural worldview (Coghlan \& Gooch, 2011; Cordero \& Rodriguez, 2009; Falk, Ballantine, Packer, \& Brinckerhoff, 2012; Morgan, 2010; Pritchard, Morgan, \& Ateljevic, 2011; Ross, 2010). Because travel situates the learner in the position of "other" and that most travel is done with a purpose for personal growth, travel (especially where one has to learn another language) is especially well suited to transformative learning (Foster, 1997; Morgan, 2010). 


\section{Chapter Three: Methods}

\section{Purpose}

The purpose of this project was to describe what elements of the transformative learning process students who participated in a month-long study-abroad program in Spain experienced. In addition, I explored what the experience of traveling to an "other" place and experiencing "otherness" had on transformative learning (Johnson-Bailey \& Alfred, 2006; Morgan, 2010). Moreover, I examined transformative learning through the critical race theory lens of Whiteness to analyze what role racial and cultural privilege played in travel for transformation.

\section{Research Questions}

In order to achieve the purpose above, the research in this project was guided by the following questions:

1. To what extent did eight students experience the elements of transformative learning during a month-long, study-abroad program in Spain?

2. What role did encounters with cultural and linguistic "otherness" play in the transformative learning (or lack thereof) of each participant?

3. What was each student's racial, economic, gender, and cultural identity? How did each student's identity impact her or his worldview and transformations (or lack thereof) during the month-long, study-abroad experience?

\section{Rationale for Choosing Qualitative Research}

A qualitative research method was necessary because observations, interviews, and open-ended survey prompts used in this study required the real-life context of a 
study-abroad program (Gall, Gall, \& Borg, 2007; Marshall \& Rossman, 2011). Research that elicits tacit knowledge and subjective understandings (as in the multiple interviews described later) and interpretations and research that delves in depth into complexities and processes (such as the multiple frames of looking at TLT through the applied travel and CRT frameworks) further necessitate a qualitative methodology (Marshall \& Rossman, 2011). Furthermore, the small sample size $(\mathrm{N}=7)$, purposive sampling technique, open-ended and informal nature of interviews, descriptive nature of individual experiences/thought processes, and grounded theory analysis of text and image data all were best accomplished and understood through qualitative research methodology (Clark \& Cresswell, 2010).

Through this study I aimed to fill a gap in existing knowledge, extend past research, and add to existing practice, which are also characteristically suited for qualitative methodology (Clark \& Cresswell, 2010; Guba \& Lincoln, 2003). Moreover, because this project was an initial attempt to explore a study-abroad program through both transformative learning theory and critical race theory lenses, the potential variables to examine were not clear before data were collected. Therefore, because this is a relatively new area of research, a qualitative research approach expanded information and increased the utility of the findings of this project (Marshall \& Rossman, 2011; Merriam \& Kim, 2012). However, it is important to note that the purpose of this proposal was not generalizability as the term is used in quantitative research (Clark \& Cresswell, 2010). Rather, the purpose of this project was to obtain deep, rich, and complex understandings that reflected the perspective of the students involved in the study-abroad 
experience in order to delineate principles of how adults learn and transform toward more permeable worldviews (Gall, Gall, \& Borg, 2007; O'Sullivan, 2012).

\section{Paradigmatic Underpinning of the Study}

In order to understand the selection of research methods and understand why a qualitative method is the best approach for this project, it is essential to situate the methods in the critical social TLT paradigms of constructivist epistemology, humanist axiology, and critical social/CRT ontology.

Constructivist epistemology. The constructivist underpinnings of TLT mean that there exist multiple, varied, subjective, and complex views--truth is relative (Guba \& Lincoln, 2003; Merriam \& Kim, 2012). Constructive research delves into the complexities and processes of how individuals think (cognitive), feel (affective), and act (conative) in society (Brookfield, 2012; Mezirow, 2012). Indeed, Merriam and Kim (2012) state that "something as personal as transformative learning and subsequent transformation lends itself to qualitative or constructivist research because we are interested in understanding the meaning of the experience for the participants involved" (Merriam \& Kim, 2012, p. 61). Moreover, the aim of constructivist inquiry is to authentically understand, reconstruct, and represent the knowledge and voice of active participants (Guba \& Lincoln, 2003). Rather than viewing information from a "scientific" approach where "truth" is seen in a logical-deductive cause-and-effect system, I constructed meaning by interpreting the interplay between the participants' thoughts, feelings, and classroom and cultural site interactions (Guba \& Lincoln, 2003; Mezirow, 2012). I attempted to ascertain the participants' thoughts through a system of 
group and individual interviews, member checks, observations, open-ended surveys, and photographs the participants took during the study (Kezar, Carducci, \& ContrerasMcGavin, 2006). Grounded theory (described in the data analysis section) was used to code and analyze the data collected and lines up with a constructivist underpinning in that the interviews and observations will be compared and viewed as constructs in obtaining reliable, confirmable, trustworthy, and transferable understanding of the salient themes and patterns that emerged as the data were collected (Guba \& Lincoln, 2003; Kezar, Carducci, \& Contreras-McGavin, 2006). The data were reviewed in order to identify and make meaning of issues that informed and were valuable and authentic to constructing how the participants' worldview changed as the study-abroad semester continues. I understand that is not possible to generalize data under such a construct, but accept that the richness of the descriptive data were more valuable at this initial stage of qualitative inquiry (Marshall \& Rossman, 2011).

Humanist axiology. Humanism values freedom, agency, tolerance, social justice, and near limitless capacity for learning in adult learners (Mezirow, 2012). Because the humanist axiology of critical social TLT views humans as inherently good, free, and autonomous, with limitless potential (Cranton \& Taylor, 2012), these values played a great deal in selecting participants for this research study. Furthermore, according to the humanist axiology, it was important that the participants be seen as adults with power over their own thoughts and behaviors by both the participants and researcher.

From a humanist axiology perspective, it was also imperative that I value the participants as "my people" to avoid essentializing, stereotyping, or "othering" them. A 
repeated critique of ethnographic studies stemming back to 1950s anthropological studies is that the very nature of studying a group other than one's own is an ethnocentric act of "othering" that population (Gold, 1958). Likewise, Laible (2000) critiques the idea of ethnographic studies where researchers study individuals with whom they do not identify, arguing that the very process of studying a group outside of one's own is a process of exoticizing the other group. Therefore, according to Laible, the researcher must identify with the group that she or he is studying. For example, I can study teachers, because I consider myself a teacher. Following is a brief excerpt from Liable's article where she articulates this positionality inside social science research:

I have continued to read scholarly work regarding outsider research... and EuroAmerican privilege.... I have also recently had frank discussions with AfricanAmerican colleagues, students, and friends regarding these topics. My participation in these activities has convinced me that I will NEVER be able to fully or accurately understand Others' experiences. My epistemology is Western, modern, and racially biased.... My desire to gaze upon the Other and reinscribe him/her in my words, in my opinion, is no longer valid or ethical. My research victimizes others, although this is not my conscious intent. p. 687 (capitalization in original)

Because I researched how race impacts one's view and desire to write a nuanced report about this variable, I can write more powerfully and in a much more nuanced way about White individuals because I identify as and am socially constructed by others as White. That is not to say I cannot be an ally or study different racial groups. Rather, it 
positions me as an insider, which provides more freedom of expression. Therefore, I selected participants with whom I share similar racial, sociocultural, and socioeconomic backgrounds. However, as will be seen in the discussion chapter, despite my passionate stance as a feminist, my identity as a male created limitations as seven out of the eight participants were female. Nervertheless, the use of member checks and multiple interviews throughout the research process (and months after the main data collection process) ensured participants had power over their own story/voice, which was a crucial element of both qualitative research methodology and a humanist axiology.

The selection of the method of photography-based narrative (described in the procedure section) as a principal and secondary data collection method also had roots in humanism. The participants were invited to share what they were experiencing from a first-person point-of-view, having autonomy over their own narrative. Additionally, it was important to analyze more than solely the words they said in interviews, or the interactions they had while I was observing their classes and cultural experiences. Sometimes words are not adequate to describe all of an individual's' thoughts and feelings. Thus, the photographs and videos that the students took included unique windows into their individual experiences that were often not captured through interviews and observations (at least for the participants that actively participated in this data collection method). Moreover, the member checks utilized after the research was over was an additional attempt to understand the long-term effects of the study-abroad program, exploring how the participants are using their agency and autonomy after the program ended. 
Critical social/CRT ontology. Many academic institutions advance literature based on principles of distance, objectivity, and rationality (Pritchard, Morgan, \& Ateljevic, 2011). However, transformative learning requires a qualitative change in the way people think, which is nearly impossible to measure objectively (Mezirow, 2012). Congruently, Brookfield (2012) states that "personal identity is not developed in a series of purely self-contained, individual choices, but is shaped by collectively generated and maintained roles, assumptions, images, and expectations associated with one's race, class, or gender" (p. 139). There exists a need to create space for "learning through the lenses of culture, diversity, and difference as well as animating awareness through the experiences and performance of the arts" (O'Sullivan, 2012, p. 173). Furthermore, the aim of research under a critical paradigmatic underpinning is to lift the voices of the marginalized (Clark \& Cresswell, 2010).

Although knowledge is constructed within individual minds, the critical social ontology of TLT required the researcher to situate the participants' and the researcher's reality within social, political, cultural, economic, ethnic, and gender variables, crystallized over time (Guba \& Lincoln, 2003). Therefore, it is not sufficient to obtain a simple "snapshot" of the participants of this study over the course of a month studyabroad program. Rather, the students were asked to connect their personal biography with societal structure and the transformations they had while participating in this research (Taylor \& Snyder, 2012). The analysis of the students' life history interviews helped contextualize the degree to which this one-month study-abroad experience served as a transformative event for each participant (Merriam \& Kim, 2012). Consequently, 
this was the reasoning behind the narrative analysis used in this research (described below in the procedure section).

Critical social ontology is decidedly subjectivist; it has a purpose of critique, transformation, and emancipation from unjust ways of seeing and acting in the world (Gall, Gall, \& Borg, 2007). Indeed, a researcher under this theoretical framework hopes to erode ignorance and motivate the participant toward political action (Guba \& Lincoln, 2003). Rather than the traditional researcher/participant role, the researcher under a critical social ontology takes on the role of transformative advocate, with a reflective, passionate, and positioned voice (Pritchard, Morgan, \& Ateljevic, 2011). Furthermore, part of the positioned voice in this research experience was a sensitivity to, and an awareness of, the transformations I underwent from sharing this amazing experience with the participants. Likewise, Pritchard, Morgan, and Ateljevic (2011) state that critical social TLT through travel "has at its heart the transformation of relationships between the researcher and the researched - no longer subjects or even participants in projects, but wherever possible, collaborators in tourism storying” (p. 952). This critical social ontology was part of the reason that I valued and utilized multiple types and opportunities to interview, observe, and perform member checks with the participants.

Furthermore, my position and participation in this research was greatly affected by being a participant observer (Gold, 1958). Brown (2009) contends that being a participant observer involves not only watching a scene but also participating in it and recording events and conversations as they occur. She additionally asserts that by actively participating in research activities during her study, she was able to corroborate 
data generated by interviewing participants as the research was conducted, which allowed the participants to play a role in the creation of themes and codes used during the grounded theory analysis of her study. This process greatly assisted the data analysis of my dissertation research as well and encouraged the participants to be active participants in generating themes. This co-creative storying is one of the ways I ensured I was getting accurate information from the participants and was able to check my personal biases as I observed and interviewed the participants.

Additionally, Ladson-Billings and Donner (2005) argue that it is the moral obligation of the researcher to advocate for social action and to encourage students toward an epistemology of emancipation, even when this call to action might harm the appearance of objectivity of the researcher (see also Delgado-Gaitan, 1993; Parker \& Lynn, 2009). Similarly, O'Sullivan (2012) calls this type of research "learning while moving" in that both the researcher and the participants are co-learning, co-transforming, and co-creating the themes to emerge through the research study. Furthermore, in the constructivist grounded theory utilized in this study, there is a tacit understanding that the relationships between researcher and participants elicits a co-construction of data, which the researcher observes and analyzes throughout data collection and analysis. Therefore, the researcher must explicitly address her or his own biases and experiences in order to understand the interplay of her or his own epistemology and the observed behavior of the participants (Charmaz, 2003). Similarly, Mills, Bonner, and Francis (2006) asserted:

Historically, grounded theory has been judged as silently authored, that is, researchers have maintained a position of "distant expert" ... Revealing the 
researcher as the author of a coconstruction of experience and meaning is an important next step in grounded theory research. p. 7

Therefore, in order to reveal my presence as researcher and the participants as coconstructors of meaning, I explicitly probe my own biases and subjectivities, as well as seek to understand how my own about how my experiences were similar to, different from, impacted by, or co-constructed with the participants of this research.

\section{Data Sources}

In order to answer the research questions, it was important to obtain data from multiple sources to obtain triangulation of data (Marshall \& Rossman, 2011). The rationale for selection, how the data were collected, and from whom the data were collected for each source will be described in detail in the procedure section. Furthermore, each of these sources was used holistically to answer all three of the research questions. Moreover, how they aligned with the research questions will also be delineated in the procedure section for each source. Finally, how each data source was analyzed will be discussed in the data analysis section. The following sources were used to answer the research questions for this study:

1) Personal Biography/Life History

2) Photo Research

3) Focus Group Interviews

4) Participant Observations

5) Informal Ethnographic Interviews

6) Secondary Data Sources: "All is (sic) data" 
7) Post-Analysis Interview/Member Check

\section{Sampling and Site Selection}

A purposive sampling technique was used in the selection of participants. Clark and Cresswell (2010) maintain that it is important to intentionally select "sites and participants based on places and people that can best help provide understanding of the central phenomenon" (p. 251). Therefore, the object in participant selection was to find a group of college students with very limited experience in visiting other cultures, who were second language learners, who were participating in a study-abroad program, and who were adults in order to match the previously explained paradigmatic framework of this study. Likewise, Marshall and Rossman (2011) state "there is a high probability that a rich mix of the processes, people, programs, interactions, and structures of interest is present" (p. 101). Similarly, this group of students and the study-abroad program were indicative of what Gall, Gall, and Borg (2007) call a theory-based purposive sample in that previous research has identified college students as particularly well prepared for critical social transformative learning. The participants were enrolled in a month-long study-abroad program, which previous empirical research has shown to be transformative (Brown, 2009). Additionally, they were traveling to a place considerably "other" than the Midwestern region where they study and live (Morgan, 2011). Furthermore, their similarities to me in racial, socioeconomic, and cultural background allowed me to portray a nuanced description and pay attention to social background and personal knowledge, but avoid stereotyping as described in the humanist axiology subsection above (Liable, 2000; Vogt, Gardner, \& Haeffele, 2012). 
The group of students selected was also a convenience sample, because I was granted access to them through a colleague who was their Spanish professor, "Dr. Green"3 (Clark \& Cresswell, 2010). Most of the students at the university in which he teaches commented to him that they had never left the state/region in which they reside. This was one of the main reasons that Dr. Green suggested that they attend this study-abroad experience in Spain. He believed that this experience would encourage the students move to a more permeable, reflexive, and critical worldview (Mezirow, 2000). One of the paragraphs from his study-abroad proposal indicated a transformative learning motive:

The program allows students to gain a cultural awareness as they interact in Spanish twenty-four hours a day, seven days a week instead of only three to five hours a week in a classroom setting. The experience in Spain diminishes inhibitions toward other cultures that students might have and provides a chance to interact with youth with different backgrounds and cultures. Beyond simply seeing the world, these students participate in it and are affected by it beyond mere tourism. As they spend time with their host families and Spanish acquaintances, students learn to see other cultures as a positive influence on their lives. That way, fear of the different or the unknown could slowly lessen for them. (Study-Abroad Proposal, p. 5)

${ }^{3}$ All personal and institutional names used in this dissertation are pseudonyms to protect the confidentiality of the research participants. The first time a pseudonym appears, it will be indicated by putting the name in quotation marks. 
When I explained the paradigmatic underpinnings of critical social TLT, he invited me to research how this study-abroad program in Spain impacted the sociocultural worldview of his students. Because acquiring access to a site for research is one of the most difficult tasks to be negotiated for social science research (Vogt, Garner, \& Haeffele, 2012), and understanding them to be well matched to the purposes of this research project, I accepted his invitation.

One negative aspect of this purposive, convenience sample is that Dr. Green was limited to the activities that were already in his prospectus for the study-abroad program, which limited the type of activities that could be added by me for the research. For example, one time I mentioned to the students that I had lived in Benidorm (a city known to have many exciting discothèques, beaches, shopping, and other tourist opportunities). Many of the participants wanted to go on a weekend when we had no cultural excursions, but the professor informed me that because the side trip was not outlined in the travel prospectus, he could not encourage them to go. He was worried if some physical or emotional harm were to happen to his participants, he might be held responsible by the university where he taught. Students, of course, being adults, were allowed to participate in whatever extra-curricular activities they desired. I, however, worried that they would see my friendship with Dr. Green as an institutional invite to a side activity. Therefore, because I did not want to create any negative situations for him or the students, I withdrew the invitation. Several similar experiences were a limitation of researching a pre-existing program rather than developing a study-abroad program myself. 


\section{Participants}

The participants all studied 2-3 years of college-level Spanish major or minor courses before the study-abroad program. There were 7 female participants (19-22 years old) and 1 male participant (28 years old). When I wrote the proposal for this research, I thought all of the participants would self-identify as White from the Dr. Green's descriptions of the students prior to the study-abroad experience. However, during data collection, I found out that two of the participants self-identified as racially White, but culturally Latina (this intersectionality will be described and discussed in the results chapter). All the students attended "Plain State University" (PSU), a public, primarily nonresidential (81\% live off campus), medium-sized, four-year university in the Midwestern United States (carnegiefoundation.org). At the time of the study-abroad program, the PSU student body was $80 \%$ White, $4.5 \%$ Latina/o, 4\% Black/AfricanAmerican, and 5\% international (princetonreview.com). Eighty-five percent of the student body received need-based financial aid (princetonreview.com). Although 69\% of the students were in-state residents, Dr. Green informed me that this number looks lower than it should be because the university is near the borders of two other states. He surmised (and data collection confirmed) that all of students were from the region (about a 150 mile $/ 250 \mathrm{~km}$ radius) surrounding the university.

\section{Negotiating Entry and Gaining Trust of the Participants}

Dr. Green informed students several months beforehand that I would be attending their study-abroad program. In addition, he secured permission for me to observe the students in their classes and participate in the cultural excursions. Furthermore, on the 
day before the university classes started, Dr. Green arranged a meeting for me to meet the participants' professors, administrative staff, cultural excursion director, international study-abroad program coordinator, and housing coordinator at "Levante University" (LU). The LU faculty and staff were extremely gracious in providing me liberal and free access to every service, cultural excursion, and class that either Dr. Green or the students had. This hospitality was an invaluable resource in accessing secondary data that could not be accomplished otherwise. Dr. Green hypothesized that this amazing access was part Spanish hospitality, part consideration for a fellow scholar, and part hope that I would take students to their study-abroad program after my degree was completed and I was in a professorial position to carry out similar study-abroad programs.

On the first day the students arrived in the city in "Levante" (the city where the Spanish university is located), there was an informal meet and greet, followed by an orientation from the housing coordinator and the cultural excursion liaison. During this meeting, I began to establish rapport with the participants, their Levante University professors, the program coordinator, the housing coordinator, and the host families. Dr. Green introduced me to the participants and allowed me to explain my research. At this meeting, I gave each participant a letter of informed consent, re-explained the research project, and informed them of their right not to participate and to withdraw from the study (or any part of the study) at any time.

At this meeting, in consultation with the Dr. Green, and in later electronic communications with my dissertation committee chair, I decided to withdraw one of the study-abroad students from this research. She came from a different university, attended 
classes in a different program, and only attended the cultural excursions with the other eight students. I felt that attending her classes (even a couple of days a week) would reduce the possibility to collect valuable data from the other eight participants because her classes happened in a different building, but at the same time as the other eight students. She was best friends with "Kylie," but was eliminated from data collection and analysis and will only be discussed in this dissertation as a secondary data source for Kylie.

During the orientation meeting, I also explained to them the Flickr photos/videos data collection method (see Photo/Video Research subheading below in the Procedure section). All of the students (except for Participant "Tess" who arrived the following day) signed the letters of consent and looked eager to participate in the research. I originally wanted to assist the students in creating a Flickr account at this meeting, but I found out that most of the students suspended their data plans while in Europe and only had internet access at the university and slow internet access with their host families. Tess signed the letter of consent, created a Flickr account, and received all of the previously detailed explanations the following day upon arrival to Levante.

In my proposal I stated, "Because the students' Spanish professor has already obtained permission for me to observe at the Spanish university and the cultural excursions, I do not anticipate problems of entry." I was correct from an institutional/university level. However, entrance was more complicated with the participants than I originally expected. There were several issues that made 
entrance/access with the students more difficult than I had anticipated when writing the research proposal.

The first difficulty was the photo/video data collection. "Kristene" seemed to obstruct this part of the study from the very beginning. Every time I would talk about it I would hear a couple of the participants laughing or see them smiling and look over and Kristene would be making the "vomit sign" by sticking her finger in her mouth or by making some other gesture of displeasure. After two days, I stopped talking about it in whole-group settings and decided to invite the students individually. All of the participants (except Kristene) signed up for Flickr over the next few days. Throughout the first four or five days, I thought Kristene was opposed to participating in the research. During the entrance interview (explained below in the Procedure section), I pulled her aside and reminded her that she had the right to withdraw from the study at any time and she informed me she simply did not like having her picture taken. I validated her concern, told her it was completely acceptable to withdraw from any part of the research, and that I would not take any pictures of her or look at any of the pictures others had taken of for my research. Her attitude and behavior regarding my dissertation visibly changed immediately following this interview. Moreover, Kristene even offered to give me her complete hand-written journal of her thoughts and experiences of the studyabroad program (which she later did) to make up for the discrepancy of not having the photo data of her for data analysis. In many ways, she was the "gatekeeper" for many of the other students to start being at ease with me (Marshall \& Rossman, 2011). 
Another access difficulty, that never completely dissipated, was my friendship with Dr. Green. They associated my research and me with him as their authority figure. While Dr. Green opened a virtual door for me institutionally, my relationship with him was a barrier that had to be slowly overcome to gain the participants' trust. Once they realized that I valued/protected their confidentiality, they began to open up much more. I perceived that the first major interview on the trip to Granada (explained in the Description of Study-Abroad Program section below) was when they mostly abandoned the fear that I was going to report everything to him. The longer the study-abroad program continued, the more their fears seemed to diminish. By the end of the second week, they even openly discussed complaints about the program and Dr. Green, which was an outward evidence I had gained more of their trust. On the return trip from the cultural excursion to Alhambra ( 8 days in to the study-abroad program), I fell asleep on the bus. The participants took silly pictures in different poses with me. I wrote in my journal that I felt that "entry had been attained" because they felt comfortable enough to joke around with me. Although their fears slowly dissipated, my friendship with Dr. Green was something that I consistently had to overcome with the participants.

The final access issue was my gender. I believe a female researcher would have been more likely to gain the trust of and build rapport more quickly with the primarily female (7 females, 1 male) group of study-abroad participants. Several examples serve as illustrations of this phenomenon. First, as I was helping the participants sign up for the Flickr application on their phones, I pointed out that their was an automatic download option that would allow them to automatically download the photographs to the website. 
Several of the participants blushed and indicated that they did not want all of their photos on Flickr. Even after I told them that they could set the settings to have them download to private and that they could then select which ones to post for everyone, they still indicated that they preferred to individually upload them one at a time. Additionally, several of the participants had boyfriends either back in the United States and one of them started dating a man from Spain while were there. I quickly became aware that it was a cultural taboo to even be in the same room or conversation while they discussed their boyfriends. I doubt that a female researcher would have encountered the same barriers to entrance. I consistently had to be aware of my positionality of maleness as I interacted with the participants.

\section{Reciprocity}

Because relationships are fundamentally important in conducting qualitative research, I sought to develop a feeling of reciprocity between the participants and me. Reciprocity was primarily obtained by consistently performing member checks so that the participants had a voice in how they would be portrayed and how their narrative would be presented. Furthermore, I sought peer review from experienced researchers throughout data collection and analysis to maintain ethical standards of research (for example, the above mentioned decision to eliminate one of the participants). In addition, the participants knew the purpose and aims of the study, how the results would be used, possible social consequences of study, that they had the right to not participate or to withdraw at any time, and that I would do as much as possible to protect their confidentiality (Clark \& Cresswell, 2010). 
Furthermore, research designs often include small tokens of appreciation (Marshall \& Rossman, 2011). Therefore, I purchased a small gift card to Corte Inglés (a Spanish department store) at the beginning of the program to show my appreciation of the participants' time (approximately $\$ 10$ ). Additionally, to avoid to appear to be taking too much of their time, I invited a participants to an horchata for their exit interview because it occurred on a day when they had a holiday from the university.

Another way reciprocity occurred was when the participants and I discussed their experiences in the program, I was open about my experiences living abroad and learning to speak in another language. I attempted to do this in a manner that influenced their responses as little as possible, but also showed them I could be trusted. Moreover, on several of the excursions, the participants met my wife and children, which was one of the most effective tools to overcome the previously mentioned gender barrier to building rapport and engendered positive feelings of trust and reciprocation.

\section{Description of Study-Abroad Program}

Each of the students selected either a three or a six credit-hour summer studyabroad experience, depending on whether they wanted to take one or two academic classes during their study-abroad program. Six of the participants chose to take both classes, while two of them only took the Levantino Art and Literature class (explained below). The students participated in classes at an accredited Spanish university, LU, Monday to Friday 8:00 a.m. to 12:00 p.m., plus additional out of class cultural/historical excursions at least weekly either on the weekends or after classes. Levante University has approximately 27,000 students, one-fourth of whom are international students, and offers 
over 50 degrees, some of which go through the doctoral level (Wikipedia.org). The students were assessed for Spanish language proficiency utilizing the Common European Framework of Reference for Languages and were all assigned to the same level, so they took the classes together as a cohort.

Spanish History and Culture (three credits). This class focused on the history, politics, and culture of Spain from pre-historic times to present. Most of the class time (the last three weeks) focused on the time period from the Spanish Civil War (1936-1939) to the time of the study-abroad program. According to the class, during the $20^{\text {th }}$ Century, Spain had a monarchy, a republic, a dictatorship, and a constitutional monarchy, along with several attempted coup d'états. The class often talked about politics, war, stereotypes of Spain/Spaniards, the brands/industries of Spain, and how different parties with different agendas portray history. The teacher, Juan, consistently discussed and invited the students to think about how the different political paradigms impacted marginalized groups in Spanish society.

Juan had a dynamic presentation style and used a variety of activities to engage students: group work, video clips, PowerPoints, student presentations, and class discussion. On multiple occasions, Juan varied from the course outline to deeper engage students in understanding the politics and history of Spain. For example, when king Juan Carlos, who had been king from 1975-2014 abdicated the throne during the study-abroad experience, one of the students asked what the red, yellow, and purple flags that people were waving in protests throughout Levante meant. Juan taught the students that this flag was the flag of the Spanish Republic and was symbolic of those particular individuals 
protesting for a republic rather than a monarchy. A discussion about Spanish politics and history ensued for about an hour, with many of the students asking questions. Another skill that Juan demonstrated was the capacity to encourage the students to reflect on United States' culture and not solely Spanish culture. For example, during the conversation above, one of the students mentioned that having a king was undesirable because he was a useless and costly figurehead. Juan encouraged the students to think about ways that the president of the United States might be seen as a "useless and costly figurehead" and solicited several responses. His class provided many opportunities to conduct informal interviews about what the students thought about Spanish politics and to ask how the culture and politics of Spain were influencing their personal paradigms.

Levantino Art and Literature (three credits). This class focused on the art and literature from the city in which the students lived during their study-abroad program, Levante. The class syllabus included a wide variety of art media: literature, poetry, sculpture, painting, architecture, photography, drama, music, film, and performance art. This class also involved understanding the historical context and politics that inspired each artist to create the different works studied in class. While the art and literature ranged from classical to post-modern, this class paralleled the Spanish History and Culture class above, wherein the final three weeks of the class were dedicated to the time from the Spanish Civil War (1936-1939) to when the study-abroad program took place.

The professor, "Ana," wrote an 800-page doctoral dissertation on the art of Levante. Her knowledge of the subject matter and the lives of the artists were impressive. Ana was enthusiastic about the subject matter, especially about the authors 
and poets studied in class. She repeatedly gave the students assignments to visit landmarks, museums, sculptures, famous eateires, and other artisans throughout Levante. Because my wife and children accompanied me on this research project, we ate many wonderful meals and saw amazing sights due to Ana's suggestions in class. However, the students did not because the events were not required for their grades. I will discuss this and other missed immersion opportunities in the Discussion section (chapter 5). Ana commented to me several times throughout the program that she had a difficult time engaging the students. During class, Ana's pedagogy was mostly a combination of lecture and whole-class question and answer techniques. She stated that she felt that only when she strayed from the theme of the class into off-topic discussions of United States Pop Culture versus Spanish pop culture would the students get excited. My observations concur with her thoughts.

\section{Cultural excursion participation (one-third of the grade for each class). It}

was additionally expected that the students would participate in a series of cultural excursions. The excursions provided students with valuable learning experiences beyond the classroom. Active participation in these cultural excursions formed one-third of the grade for each of the classes. Both professors did an excellent job of including the excursions in the classroom discussions.

One the first official day of classes, LU hosted the students for a reception supper (la comida is the biggest meal of the day, eaten at approximately 2:00 p.m.; "supper" is the best translation for that term). Juan, the program administrator, Dr. Green, and I accompanied the students. The chef displayed a variety of appetizers that were typical to 
the region surrounding the university. The chef announced each new dish that he brought out, explained the history of it, and invited the students to partake. In addition to the food, there were local wines, beers, and fruit drinks representative of the region surrounding Levante.

On the first Friday of the study-abroad program, the participants boarded a bus to go to Granada. This trip included an official tour in Spanish of the Alhambra (a Moorish fortress, mosque, and later palace for Charles V of Spain), a city tour of the Albaicin (the historical center of Granada), and a Flamenco Tablao (flamenco music and dancing). The participants enjoyed two different tour guides for this trip: "Manolo," who was the guide from the time the bus picked us up in Levante to the end of the excursion, and "Eva," who was our tour of Alhambra. Eva had a master's degree in medieval art and architecture. She took us on an extensive tour of the Alhambra, explained both the Muslim and Christian influences, and explained the practical and religious reasons behind the art and architecture.

The following week, students boarded a bus to experience a walking city tour of Alicante, Spain. This guided tour included a visit to the Castillo de Santa Bárbara (a castle and fortress from the 9th Century) and the Barrio de Santa Cruz (the historical center of Alicante). The purpose of this excursion was to provide students with an historical and cultural perspective of the city of Alicante and its people. Alicante was the final stronghold against Franco in the Spanish Civil War. The tour guide explained the many different religious, political, and governmental groups that had used the fortress from the $9^{\text {th }}$ Century through the Spanish Civil War. 
The third week the students went to the Museum of Modern Art (MOMA) in Levante. The students were shown works by many famous Spanish artists. However, the emphasis was on Levantino artists and sculptures. Although the tour guide was not as dynamic as previous tour guides, she did tie in the paintings and sculptures to artists that the students were studying in their Lavantino Art and Literature class.

During the final week, the students also attended a bullfight in Levante. After the students had watched three bulls get fought and killed, Dr. Green gave them the option to leave. About half of the participants left and half stayed. Dr. Green said that the bullfight is always the most polarizing cultural excursion of the study-abroad program. As will be seen in the Results chapter, participants had the strongest reactions to this of all of the cultural excursions. I asked Dr, Green why he included this in the experience and he said that he felt that it gave the students a chance to think about the "barbarities" of United States culture. He also mentioned that there are already two autonomous communities where bullfighting is outlawed in Spain and said that his students might be the last generation to see this tradition that goes back thousands of years.

Immersion in culture. Each of the students was assigned a host family upon arrival in Levante. They were required to eat dinner with the host family and interact with them daily as part of their cultural excursion grade. Because students stayed with host families, the expected degree of immersion in culture was high. Plus, because participants in the program study at a large Spanish university, it was anticipated that their experiences become enhanced by their daily interactions with Spanish and/or international students. Moreover, students were expected to speak Spanish both inside 
and outside of class. Usage of Spanish was also expected during the mandatory cultural excursions. Furthermore, during the guided learning experiences and cultural excursions, the guides only used Spanish in order to give the students an opportunity to listen and ask questions in Spanish. The benefits and limitations of, and the degree of, immersion in the Spanish culture will be addressed in the Discussion chapter.

\section{Research Procedure}

This was a critical culture-based study that borrowed from many traditions as is common with CRT and critical theory research (Bell, 2009; E. Taylor, 2009). Gall, Gall, and Borg (2007) state that "more than any other qualitative research tradition, cultural studies reflect a blending of disciplinary traditions" (p. 509). This research design borrowed from multiple academic disciplines: phenomenology, ethnography, grounded theory, and critical cultural studies. From phenomenology, I borrowed the focus on the individual experience and construction of each individual participant, as well as the need to constantly probe my own biases and subjectivities as a researcher (Marshall \& Rossman, 2011). This focus on the individual also ties in to the constructivist underpinning of this research project.

Many of the data collection methods (in-depth interviews, deep immersion in the culture, extensive observations with a focus on culture, and informal interviews based on the observations) stemmed from ethnographic traditions in qualitative research (Clark \& Creswell, 2010). The ethnographic collection of data through observations and informal interviews allowed the participants to feel comfortable to express their thoughts and feelings, which characteristically is inclined toward qualitative research (Brown, 2009). 
Ethnographic observations and interviews allowed the researcher to get detailed, subjective (personal), and in-depth information from research participants on lived experiences (Vogt, Gardner, \& Haeffele, 2012). Furthermore, this method aligned with the humanist axiology of this study wherein I saw the participants as "my people" to avoid essentializing, stereotyping, or "othering" them (Liable, 2000). Therefore, I attempted to select participants with whom I shared similar racial, sociocultural, and socioeconomic backgrounds in order to provide a nuanced description of the events as they naturally unfolded.

In order to ensure trustworthiness of the data collection and analysis it was important to collect as many useful kinds of data possible utilizing multiple methods (Vogt, Gardner, \& Haeffele, 2012). This was rooted in both the qualitative nature of this study (Marshall \& Rossman, 2011) and the use of grounded theory to analyze data (Charmaz, 2003). Therefore, this dissertation project incorporated the following methods of data collection:

Personal biography/life history. Many scholars are using new techniques to collect transformative learning data outside of traditional observations and interviews (Cranton \& Taylor, 2012). One of the ways that researchers are starting to understand transformative learning is through the use of narrative analysis, wherein participants connect personal biography with societal structure (Taylor \& Snyder, 2012). Telling stories is one of the oldest ways to pass information along to others and interviewing is perhaps the oldest way to elicit stories from others (Vogt, Gardner, \& Haeffele, 2012). Indeed, Gall, Gall, and Borg (2007) state, "life history is the study of the life 
experiences of individuals from the perspective of how these individuals interpret and understand the world around them" (p. 498). Thus, narrative research captures stories which are an everyday form of data used by most people; the researcher reports chronology, context, and themes from the stories (Clark \& Cresswell, 2010).

To describe this method, Merriam and Kim (2012) maintain, "narrative analysis is quite simply the analysis of people's stories" (p. 63). In addition, narrative analysis aligns with the critical race theory (CRT) use of stories/counter-stories to highlight the voices of the people being studied (Bell, 2009; Ladson-Billings, 2009; Solorzano \& Yosso, 2009; Taylor, 2009). Autobiographical narratives allow the past to inform the future and bring the present into presence, which contextualizes the participants' current feelings even as they relate to past events (Johnson, 2003). Merriam and Kim (2012) note, "narrative analysis is particularly well suited for the study of transformative learning because it allows people to convey their personal experience of this type through stories" (p. 63). Narrative analysis creates a particularly rich approach to understanding transformative learning because one problem with traditional qualitative research is that the research provides only a brief snapshot of the thoughts and behaviors of the participant as they are seen through the eyes of the researcher (Taylor \& Snyder, 2012).

Marshall and Rossman (2011) explain the benefit of using biographical narratives could be especially helpful in a study like this one where participants were being asked to express their experiences both as individuals and as a group trying to adjust to a new culture: 
Life histories ... assume a complex interaction between the individual's understanding of his world and that world itself. They are, therefore, uniquely suited to depicting and making theoretical sense of the socialization of a person into a cultural milieu. Thus, one understands a culture through the history of one person's development or life within it, a history told in ways that capture the person's feelings, views, and perspectives. The life history is often an account of how an individual enters a group and becomes socialized into it. That history includes learning to meet the normative expectations of that society by gender, social class, or ... culture. (Marshall \& Rossman, 2011, p. 151)

It is important to realize that qualitative research will always be filtered and coauthored through the researcher and her or his experiences (Merriam \& Kim, 2012). It is the researcher that typically brings forth the themes that emerge through a qualitative study. However, one of the benefits of narrative analysis is that it encourages the participants to be involved in the generation of emergent themes and patterns, urging them to become co-collaborators with the researcher in data collection and analysis in participative storying (Pritchard, Morgan, \& Ateljevic, 2011; see also Vogt, Gardner, \& Haeffele, 2012).

The cultural excursions to the different historical sites provided excellent scenarios to ask the students to connect their personal biographies to their experiences in the study-abroad program. The first excursion to the Alhambra was particularly wellsuited to this type of connection because it took about 5 hours by bus each way. This time allowed me to get the participants" "stories" of who they are, their backgrounds, and 
why they participated in the study-abroad program. As noted previously, the Alhambra excursion happened on the first Friday of the study-abroad program when the students had been in Spain for either five or six days. This timeline was sufficient for me to have mostly established successful entry and rapport with each of the participants (Marshall \& Rossman, 2011), but also was early enough in the program to get their initial ideas of the culture, their host family, and their experience at LU.

Detailed interviews are the primary data collection for grounded theory (Clark \& Cresswell, 2010). Typically, qualitative semi-structured interviews include general, broad questions that the researcher will ask the participants (Vogt, Gardner, \& Haeffele, 2012). It was important in this research design, however, that the questions be sufficiently flexible to allow for changes in individual styles of telling stories and to elicit information from different personality styles (Marshall \& Rossman, 2011). Also, if an answer was unclear or I needed to ask follow-up questions, it was important to have enough freedom to vary the questions according to the needs of each participant. Following is a list of the type of questions that I asked participants on the cultural excursion to Granada:

- Why did you decide to come on this study-abroad program to Spain?

- Why here, why now?

- What was your process for deciding to come here?

- Since you've been here for 6 days, what differences do you notice (in general) between Spain and your home?

- How does your family feel about you being here? Tell me about them. 
- Tell me about your experience with your host family for these first 6 days.

- How is your host family different from your family of origin?

- What cultural differences have you already noticed?

- Have there been any surprises or disappointments so far?

Photo/video research. An additional area currently being used to generate more specific and personal data for transformative learning is having the participants take photographs and videos of the travel experience (Merriam \& Kim, 2012). Digital storytelling is a new approach to narrating personal narratives that draw on the power of digitized images to supplement text data with visual and sound information (Clark \& Cresswell, 2010). Typically, the participants are then interviewed to encourage them to express their thoughts about the photographs (Cranton \& Taylor, 2012; Taylor \& Snyder, 2012). Moreover, Goulah (2007) asserts that having the participants collect data through photography and video is one of the most efficient, yet underutilized and undertheorized methods of data collection. Goulah (2007) also found that having students create their own media empirically improved their capacity to communicate in a foreign language and interact with the host culture.

Emerging in the 1990s, using photos and videos to allow the participants to generate their own stories has its roots in emancipatory ideology because it allows them to give voice (and visual/audio) to their lived experiences (Marshall \& Rossman, 2011). While photography has been used for decades as a tool for documentation, research, and education, Clover (2006) maintains that the power under these circumstances always lied in the hands of the researcher rather than the participant, causing the voices of and 
knowledge of the participants to be silent or objectified. She argues that by putting the camera into the hands of the participants, the researcher can create "participatory photography" wherein the participants can promote dialogue, engage in activism, and challenge the viewer to reflect on the social context of a situation, thereby promoting the voice of the participant (see also Goulah, 2007). Clover (2006) also states, "participatory photography places the medium of the camera into the hands of learners to democratize the image-making dynamic and give them the power to show and speak their own realities" (p. 275). Furthermore, Merriam and Kim (2012) maintain that arts-based research (like photography and videography) helps a TLT researcher understand the "affective, intuitive, relational, and often irrational ways of knowing beyond the limited cognitive perspective" (p. 64). Tisdell (2012) also argues for encouraging participants to take an active role in generating data for research: "we teach the importance of critique almost as if this is a form that transforms their thinking, but we do not invite learners often enough to call upon the wonder of their own creativity, which is important in cultivating hope, possibility, and action" (p. 27).

Scarles (2012) cautions that the same concerns that affect travel for transformation (see Curry-Stevens, 2007; Pritchard, Morgan, \& Ateljevic, 2011) apply to photography and videography: the host culture should be respected, and not exoticized or othered in the process. The purpose of these photos was to attempt to understand the thought process of the participant and to give the researcher a starting point for interviewing the participants rather than to represent the host culture. Therefore, the focus was on the student, not on the host community. Although the photographer has the 
power in taking photos, she or he should avoid essentializing, stereotyping, or marginalizing the host culture. Scarles (2012) notes, "photography is not an end in itself and reflexive questioning of the relationship between the photographer and those being photographed is vital" (p. 929). From my observations, throughout the study-abroad program, I noted that the participants naturally avoided taking photos that might be seen as essentializing. Rather, their pictures almost always involved their interactions with, rather than their exploitation of, the host culture. As will be explained in the Results chapter, many of the students feared looking like tourists, so they were extremely careful to make sure it was appropriate to take pictures before they took them.

From a practical standpoint, much of this study relied on ethnographic observations, which asks the reader of this research project to trust in my observational skills. By putting the camera into the hands of the participants, they helped capture special moments in time through their cameras that I could later interview them about and ask them what they meant. This not only encouraged their voices to come forward, but helped generate more data. For example, I was asked by Dr. Green to limit my contact with the participants' host families because they were not official parts of the investigation. However, many of the students took photos of their interactions with their host families, which opened the possibility to interviewing the participants about their relationships and experiences with their host families without me interfering with the valuable time of the host families. Therefore, the participants become co-researchers, generating images and causing themes to emerge for later discussion and analysis (Merriam \& Kim 2012). 
It is imperative to recognize that the focus of these photographs was not the actual content of them. Rather, from the constructivist grounded theory method utilized in this study, the focus was to seek to understand what events, people, experiences, and places the participants were seeing, documenting, and reflecting on (Charmaz, 2003). It was the participants themselves that were the center of this method, rather than the subjects or other content of the photographs.

In order to collect these photos into a manageable format and to limit the time that the students were involved in taking/submitting photos, I asked them to share their photos via Flickr.com. There were several benefits to this particular format. First, Flickr provides users one terabyte of free space, which made it so that the students and I did not run out of free space. Moreover, Flickr is very easy to set up (5 minutes per participant), is secured by password protection, gives the user control over how and with whom the photos will be shared, and can be set up with most internet connected devices to automatically upload. Furthermore, like other social media, Flickr allows (even encourages) users to make comments on the user's photos and the photos of others that are in a group. However, very few of the participants took advantage of this feature. Post-study analysis yielded little results of interactions between research participants on this site. Rather, most of the comments on this website were questions to me about how to use or how to unlock the photo settings so that the rest of the group could see them.

Additionally, in this dissertation, there was a large disparity in the amount of data collected from the photos of the participants. For example, "Tim" and Kylie uploaded hundreds of photos to Flickr, while most of the students only uploaded a few (as 
mentioned earlier, Kristene never signed up for Flickr). However, during the studyabroad experience, it was providential that the students who talked the least in classes and cultural excursions (Tim an Kylie) were the participants who took the most pictures and uploaded them to Flickr. Despite the disparity in numbers of photographs taken, the photos tended to be of all of the participants, even though the point-of-view was largely that of two of the participants. Ultimately, these photos were invaluable artifacts that allowed me to interview all of the participants (except Kristene, to whom I promised I would disregard in the photos) about what was happening outside of class time and the cultural excursions.

I used constructivist grounded theory (described in the data analysis section) to analyze the photos both individually and collectively while in the field. While other scholars claim the comments and photos could serve as data points to be analyzed later (Goulah, 2007), I decided to only use the photos to spur more informal interviews during data collection. The disparity in numbers of photos and the relative lack of substantial comments rendered the photos and videos useless for post-study data analysis.

Nevertheless, the photographs/videos represented an ethnographic observational snapshot at a moment in time of the experiences of the participants and provided prompts for informal interviews during the study-abroad program, rather than as a data analysis source following data collection. Additionally, many of the photos were taken at moments when I was not with the students in classes or on excursions, which widened my knowledge of what they did in their recreational time and with their host families. In the constructivist grounded theory methodology utilized for data analysis, it was 
imperative to be open to the generation of codes as the data were collected and to include the participants as much as possible in participative co-storying (which will be discussed in the Data Analysis section below). Thus, the photos/videos were a medium for asking questions and creating informal interview opportunities. Questions asked about the photos tended to be along the following template:

- Why did you choose to take a photo of that subject?

- How did it make you feel when you saw that?

- What does that photo convey about Spanish culture?

- What does that video show about American culture?

Focus group interviews/observations. The use of focus groups is increasing in qualitative research methodology, because qualitative "researchers are finding that the interactions among the participants stimulate them to state feelings, perceptions, and beliefs they would not express if interviewed individually" (Gall, Gall, \& Borg, 2007, p. 245). The strengths of focus-group interviews are that this method is socially oriented, studies participants in a naturalistic atmosphere, and creates a more relaxed setting than a one-to-one interview (Clark \& Cresswell, 2010). Other strengths of focus-group interviews are that they are good for brainstorming and getting participants talking, which can lead to more rich, detailed data collection in individual interviews later (Vogt, Gardner, \& Haeffele, 2012). Furthermore, focus groups can be particularly helpful in understanding social dynamics or collective influences on beliefs, attitudes, or emotions, and social processes of change (Vogt, Gardner, \& Haeffele, 2012). Moreover, when combined with participant observation, focus-group interviews can be especially useful 
for gaining access, focusing site selection and sampling, and even for checking tentative conclusions (Morgan, 2010). As with other types of interviews, the format allows the facilitator the flexibility to explore unanticipated issues as they arise in the discussion (Marshall \& Rossman, 2011). It is equally important that the researcher creates a supportive environment, asking focused questions to encourage discussion and the expression of differing opinions and points of view (Marshall \& Rossman, 2011). One negative aspect of focus groups is that an overly talkative participant can sidetrack the conversation or take up a majority of the time speaking.

Dr. Green had a built-in weekly session where the students came together to discuss their experiences in the study-abroad program. In this meeting, students expressed feelings about their host family situations, their struggles or successes with Spanish proficiency, and how their professors were treating them. In addition, they reviewed previous cultural excursions and discussed upcoming ones, discussed activities that they were participating in outside of the program, and where Dr. Green ensured that they were healthy and well established. He habitually opened up the conversation to any questions or concerns that the students had. Dr. Green also encouraged me to stick around after the official meeting, because the students often expressed concerns to him after the "official" meeting terminated. These meetings were excellent resources, because the participants often explained cognitive dissonance and disorienting dilemmas they were experiencing.

In the field, I quickly discovered that these meeting were brief (most lasted about 15 minutes). I chose to treat these meetings more as observations than actual focus group 
interviews. I sat in the back of the room and quickly typed the concerns, reactions, and thoughts of the participants. Dr. Green expressed an apprehension that the students would not open up if recorded, and he had a professional obligation to ensure their physical and emotional welfare. I agreed to not record and transcribe the meetings. Consequently, I resorted to meticulous field notes using the dual column (or dual font) note-taking process described below in the Observation section (Marshall \& Rossman, 2011)

A few days before the third Monday, I asked the participants if I could have an hour of their time after this meeting to conduct a recorded, "official" focus group session after Dr. Green's health and well-being weekly interview. They all agreed. I recorded these interviews and transcribed them for data analysis. As a researcher, I was excited when after the focus group interview was complete, all of the students followed me to the tram to ask if they could continue discussing the questions. I recorded another 30 minutes of discussion. The following questions were written in my field notes for a template to the focus group interview:

- What changes have you seen in your relationships with your host family this week? Your attitude toward them/their attitude toward you?

- What were your reactions to the MOMA (modern art museum)? Anything exciting/surprising/disappointing?

- We've talked about the Spanish Civil War, First and Second republics, politics, censorship and other liberties, abdication of the king a bunch in both classes this week. Is there anything exciting/surprising/disappointing about 
the politics here in Spain? Any thoughts about how war affects a country?

- Have you made any friendships with anyone outside of your classmates? Tell me about it.

- In individual interviews (between classes, after class) I would like to ask about your weekend activities. (Medieval festival? New beaches? New museums? New places? Bodega de Vino? Tabarca? Benidorm? Madrid?) Anything surprising or disappointing?

"Anne" and Kylie did not say anything during the focus group interviews (they were both typically quiet in full-group activities), so I interviewed them individually (asking the same questions) during breaks the following day. Both of them were very open during the individual interviews. In addition, Kristene told me during the focus group interview that she went on a weekend excursion by herself to Madrid, so I performed a follow-up interview about her trip the next day as well. The rest of the students felt they had expressed everything in the focus group interview and on the tram.

Participant observations. Observation is central to qualitative research (Marshall \& Rossman, 2011). Furthermore, observational research permits the researcher to collect information of diverse aspects of lived experiences from settings, situations, practices, processes, and other social phenomena as they unfold in their natural settings (Vogt, Gardner, \& Haeffele, 2012). Additionally, the researcher is able to discover recurring patterns of behavior and relationships (Marshall \& Rossman, 2011), which is especially helpful for when participants have difficulty verbalizing ideas (Clark \& Cresswell, 2010). 
The principal benefit of observation research is the capacity for the researcher to view phenomena in their natural setting or context as they unfold (Merriam \& Kim, 2012). I observed the students in all of their classes and cultural excursions. As mentioned earlier, Dr. Green secured permission for me to observe the students in classes and participate in the cultural excursions prior to the study-abroad program. Theoretically, the frequency (daily) and duration (five or more hours daily for a month) of my observations in the classes should have reduced the effect of the observer on the observed (Gall, Gall, \& Borg, 2007). Correspondingly, I observed in my field notes at the beginning of the second week that I had become a "fly on the wall." What I meant in my field notes is, even though the participants interacted with me before and after the classes and during the cultural excursions, they stopped noticing that I was there and acted more and more naturally the longer the study-abroad program continued. I also observed the same phenomenon with the professors, Juan and Ana. It took about a week before their body language and speech looked completely natural.

As I attended the classes and participated in the cultural excursions, I utilized a dual font note taking strategy during the observations when writing in a field observation journal, based off Marshall and Rossman's (2011) dual-columned note taking strategy. However, I made one small adaption. With the electronic formats I used for taking field notes (computer in classroom observations and mobile phone elsewhere) I found it easier to change fonts rather than change columns. In regular text, I attempted to write down descriptive observations that related to what was happening in the classroom, what the professor was saying, what the students were saying, and their reactions to the 
classroom methods. Gall, Gall, and Borg (2007) state that these descriptive observations should contain "verbal portraits of the research participants, reconstruction of dialogue, description of the physical setting, accounts of particular events, and descriptions of the observer's behavior" (p. 281). In italicized text, I wrote down personal insights and initial emerging analytical comments that demonstrated elements of transformative learning. Gall, Gall, and Borg (2007) state that these reflective insights should include the "researcher's personal account," including "methods of data collection and analysis, ethical dilemmas and conflict, and the observer's frame of mind, along with emerging interpretations" (p. 281). Annotating personal reflections, insights, conflicts, and emerging themes also aligns with the constructivist grounded theory approach (described in the Data Analysis section below) utilized for data analysis in this study. This style of note taking is also described by Clark and Cresswell (2010) when they state that field notes are both descriptive and reflective: descriptive in that they "record a description of the observed events, activities, and people" (p. 162); reflective in that they "record personal thoughts that researchers have that relate to their insights, hunches, or broad ideas that emerge" (p. 162). Another benefit to this method was it provided opportunities to check in with my dissertation committee chair when I had ethical concerns or questions about proper data management.

I also consistently wrote down questions to ask the participants after class in informal interviews (I used bullet points to make the questions stand out to remind me to ask the questions later). In addition, when helpful, I took photos, recorded voice memos to myself, and wrote prompts to write about later in Evernote (described in more detail in 
the Data Analysis section below) so that I could more fully write about the observations later. About every other day, I went in to my notes and wrote detailed explanations and personal insights to these prompts. During these journaling sessions, I additionally went over other field notes taken and filled in any missing parts to the experiences to ensure complete information during data analysis. These sessions also provided opportunities to write down questions for member checks with the participants to solicit further information during informal interviews. Later, I performed informal interviews (see below) for anything that might require further insights. Special attention was paid to elements that aligned with the three research questions regarding worldview transformations, how the travel experience affects transformation, and how sociocultural privilege might affect transformation. However, I attempted to be open to additional themes or questions that arose during data collection, which is a research imperative in all qualitative research, but especially in constructivist grounded theory (Charmaz, 2003; Marshal \& Rossman, 2011). The analysis of the field notes and their corresponding informal interviews will be described in the data analysis section below.

It is important to acknowledge that many of the observations occurred in Spanish. I have a degree in Spanish translation with a specialty in Spanish to English translation. I additionally lived in the region of Spain where LU is located for more than two years and am familiar with the idioms, customs, and dialects of Spain. However, all translations are biased as they pass through the mental construct of the translator. My primary method for reducing this bias was to perform consistent member checks with the participants, the professors, and the tour guides to ensure that what I heard was what was intended. 
Furthermore, this helped because most of the participants had limited vocabulary and Spanish proficiency, so I could make sure what they said was what they thought they said. Moreover, I developed very cordial relationships with the LU professors, staff, and excursion tour guides so I felt comfortable soliciting further insights or information when needed.

Informal ethnographic interviews. Interviews are one of the best methods for getting a participant to explain her or his ideas and behavior (Marshall \& Rossman, 2011). Typically, interviews that go along with ethnographic field observations are unstructured (Vogt, Gardner, \& Haeffelle, 2012). These interviews occur when a researcher desires further information from a participant about something observed during the day. Consequently, the questions typically include broad, general questions that arise from the observations and other data collection methods, and are described and then analyzed later for themes (Clark \& Cresswell, 2010). Moreover, interviews allow the researcher to get detailed, subjective (personal), and in-depth information from research participants on lived experiences (Vogt, Gardner, \& Haeffele, 2012). Furthermore, it is important for these interviews to be as short as possible to avoid taxing the participants' patience by requiring too much of their time (Gall, Gall, \& Borg, 2007). Another added benefit of keeping the interviews short and informal is it allows the researcher to obtain information as quickly as possible after the observed event (Marshall \& Rossman, 2011). One weakness of this format is that the unstructured interviews rely on the knowledge and ability of the "researcher to think on his feet during the interview" (Gall, Gall, \& Borg, 2007, p. 249). 
Due to the constructivist underpinning to this research methodology, soliciting explanations through informal interviews was crucial to understanding the thoughts and transformative learning of each participant. Multiple times per week I conducted informal interviews with each participant to discuss questions that arose during observations. These informal interviews occurred as soon as was reasonably possible after the observed phenomenon (usually directly after the class in which the question arose or in a break during cultural excursions). Additionally, I conducted informal interviews when I saw a picture on Flickr that required more information before class started the following day. Moreover, if something happened during class where a participant's behavior was out of the norm, I discussed her or his behavior during a class break.

Note-taking and audio recording are the typical ways in which interviews are preserved (Gall, Gall, \& Borg, 2007). Dr. Green believed that audio recording these brief, informal interviews would inhibit casual, uninhibited speech. Many times he was correct. Taking out the audio recorder would have limited casual exchanges between participants. However, the participants quickly overcame any inhibitions about the audio recorder. When their thoughts or insights were brief or when I observed an interchange between the students, I used my phone or computer to quickly annotate any observations that I had during the interview (Marshall \& Rossman, 2011). Immediately following the interviews, I "cleaned up" the notes by writing down everything I could remember about the interview (transcribe as much as possible of the conversation, note body language, posture, behavior, etc.) (Marshall \& Rossman, 2011). For any insights that were going to 
last more than a minute or solicit deep emotions, I recorded the informal interviews with an audio recorder. Within about a week, the participants developed an inner sense of what type of information I recorded versus what type I annotated in my field notes. An inside joke became common among the participants during informal interviews where they would say, "you're going to need to take out your audio recorder for this one" when they knew that they wanted to talk about deep emotions or when they were going to tell me a story that took more than a minute to explain.

Because of the informal and spontaneous nature of these interviews, creating a question protocol in advance was impossible. However, the questions involved a mix of what Marshall and Rossman (2011) call descriptive, structural, and means-end questions. Descriptive questions are broad, general questions that get students talking about daily activities and the people in their lives. Structural questions discuss how participants categorize and systematize cultural knowledge in their brains. Means-end questions elicit meanings behind the participants' behaviors and events. Marshall and Rossman (2011) maintain that by combining the three types of questions, the researcher can describe rich narratives, formulate working hypotheses, highlight the nuances of culture, and avoid over-simplification in description and analysis. I found their three types of questions to be an effective template for generating quality informal interviews.

Like mentioned in the previous section, this research methodology requires a mix of Spanish and English. I conducted all interviews with the participants in English to reduce the cognitive load and to allow them to freely express ideas and thoughts in their native language. However, this system also had drawbacks. Dr. Green pointed out that I 
became an escape for the students any time they were overwhelmed with speaking Spanish. He mentioned to me that this particular group spoke more English outside of class settings than any of his previous groups. He felt that the "othering" of learning a new language and immersion in the host culture suffered because of this data collection method. Nevertheless, due to the limited Spanish proficiency of the participants, we agreed that this was the most effective method for understanding the thoughts, intentions, and feelings of the participants.

Additional data sources. When doing research that involves either ethnographic collection of data or grounded theory analysis, it is important for the researcher to view everything as a possible rich source of data (Charmaz, 2003). Therefore, in addition to the primary sources of data listed above, I will draw data from as many sources as possible during the data collection phase of this research project. Some secondary possible sources of data may include: my own journal of the events of this program as well as the insights I have about the participants, their professors at the LU, host families, tour guides on the cultural excursions, Dr. Green, the cultural coordinator from the Spanish university that coordinates host families and cultural excursions, and just talking with everyone with whom the students interact.

Additionally, I am going to ask the students if I can take pictures of them to use to remind myself to ask them questions during the weekly focus group interviews and the informal ethnographic interviews. Marshall and Rossman (2011) give the following counsel about researchers photographing research participants: 
Do the participants know that photos or videos are being made? Are they fully aware? And most important, have they given their consent to be represented in a photo or in a video? Especially problematic with visual representations of people is the recurring question of protecting their identities. Furthermore, these representations, once digitized, may spread without the researcher's knowledge. In the proposal, the researcher should indicate how she will protect the identities of the participants, scrupulously, and how well she is prepared to use these media ethically and sensitively. (p. 185)

Following this counsel, I included in the letter of consent how I plan to use the photographs, who would have access to see them, and include an opt out option for those who do not want their photograph taken. Only Kristene opted out of this portion of data collection.

I was also given a copy of the students' pre- and post-trip evaluations of the study-abroad program. Probably the greatest advantage of using documents and other artifacts is that it does not disrupt ongoing events; these materials can be gathered without disturbing the setting (Marshall \& Rossman, 2011). Unfortunately, the pre- and poststudy-abroad evaluation documents were provided by PSU. Therefore, I did not have any control of the questions or the format of it. However, I was granted the access to use the pre- and post-surveys as data. Dr. Green additionally asked them to take their time filling out the forms honestly because it will be used to inform my research. I include both surveys, as well as the email permission to use them in Appendix A. To protect the confidentiality of the participants, I removed any information that identified the 
university. I also removed spaces to answer the questions in order to make them easier to read. This questionnaire gave some insights to the students both pre- and post- studyabroad plans, desires, goals, and socio-economic status. It was also very interesting to compare the thoughts about culture and language before and after the study-abroad program. Unfortunately, the documents were anonymous, which rendered them useless for analyzing the thoughts and transformations of individual participants, but gave me insights as I analyzed overarching themes for the group.

Post-analysis interview/member check phone interview. Member checking is the process of reviewing interview transcripts, other data, and analysis with participants so they can ensure that the researcher has not misrepresented them in any way (Vogt, Gardner, \& Haeffele, 2012). Approximately five months after the study-abroad experience (and after immersing myself into and analyzing data), I sent a summary sheet to each of the participants that included themes that emerged from their experience as an individual and a group. It also contained a summary of key moments or interviews with each participant. Furthermore, I included questions where I desired further information from the participant in the summary sheet as well. Because of the large geographic distance between the participants and me, I had to conduct these interviews over the phone. Phone interviews save greatly on cost, but make it impossible to see the body language of the participants (Clark \& Cresswell, 2010). Therefore, with students who feel comfortable using a video phoning service, I used FaceTime to overcome this disadvantage. Six out of the eight participants were able to use FaceTime. I recorded all of the interviews and transcribed the conversations. However, with one of the 
participants ("Becky"), the recording never started. Immediately after the interview, I went through the questions and wrote down every possible thing I could remember. In addition to the member check, I carried out a follow-up interview about "reverse culture shock." Reverse culture shock is where students' experiences during study abroad make it problematic for the student to understand elements of the home culture or for people at home to understand the student upon re-entry to the home culture (Brown, 2009; Coghlan \& Gooch, 2011). For example, I still experience reverse culture shock every time I am asked for my insurance card before a doctor agrees to see me as a patient 15 years after living in a country with universal health coverage.

Typically, qualitative semi-structured interviews include general, broad questions that the researcher asks the participants (Vogt, Gardner, \& Haeffele, 2012). It is important, however, that the questions be sufficiently flexible to allow for changes in individual styles of telling stories and to elicit information from individuals that are different (Marshall \& Rossman, 2011). Also, if an answer was unclear or I needed to ask follow-up questions, it was important to have enough freedom to vary the questions according to the needs of each participant. Following is a list of the types of questions that I asked participants to ensure that I represented them correctly and to ask about reverse culture shock:

- As you know, I was conducting research on the ways you may or may not have changed during the time we spent in Spain. What in this summary do you think I got right? What in this summary would you like to clarify for me? Is there 
anything that you think I got wrong? If so, how would you change what I've written?

- Looking back, what themes do you see as you reflect upon your trip?

- Has anyone around you mentioned that you're different in some way? What way? Are they right?

-What new things did you notice when you returned home? Did anything feel strange or different to you? What new things did you notice when you returned home?

- If you could do this trip all over again, is there anything you would do differently? Any advice you would give someone going on this trip in the future?

\section{Researcher as Instrument}

Reviewing transformative learning theory over the last five years helped bring about a disorienting dilemma and transform my attitudes and change my behavior. As a White, Christian, heterosexual male, I have become more aware that economic, governmental, linguistic, political, psychological, and educational systems are designed to give me a position of privilege (Mezirow, 1998, 2000; McIntosh, 1990) over other races (Crozier, 2003), ethnicities (Ladson-Billings, 2003), nationalities (Delgado-Bernal, 2002), sexual orientations (Gamson, 2003), belief systems (Blumenfeld, 2006), cultures (Writer, 2002), genders (Marshall \& Rossman, 2011), transgender individuals (Adams, et al., 2010), and classes (Jensen, 2004; Smiley \& West, 2012). This position of privilege largely allows me to escape victimization, harassment, and discrimination, whereas 
TRAVEL FOR TRANSFORMATION

marginalized members of society must confront their lack of privilege daily in order to survive (O’Sullivan, 1999).

Notwithstanding my privilege, I choose to actively seek methods to create a more inclusive worldview and eradicate inequities that are within my power to change. I realize that economic, governmental, linguistic, political, and many other organizational systems are typically out of my capacity to change. As an educator and student, however, I have access to educational processes and institutions. For transformation to come about in large-scale organizations and systems like education, people occupying positions of privilege, like me, will have to act first in order to break down existing barriers to equality (O'Sullivan, 1999). However, ignorance of privilege is so intrinsically systematized that change will need to occur within individuals first (McIntosh, 1990). Transformative learning theory (Mezirow, 1996, 1997, 1998, 2000, 2004) is a framework that invites individuals to confront their privilege and eliminate extant obstacles to equity. I have become conscious of my role as an observer of marginalization and have come to understand that action is required of those who observe social injustice (Delgado-Gaitan, 1993; Kezar, Carducci, \& Contreras-McGavin, 2006; Ladson-Billings et al., 2005). It is my responsibility to be an ally to the marginalized and alienated members of the classrooms in which I participate as a student, teacher, or researcher and work to transform the attitudes and behaviors of the dominant majority (Ladson-Billings \& Donner, 2005).

My research paradigm is saturated with a critical cultural ontology, wherein it contains a dialogic approach to ensure sensitivity to lived experiences and narratives of 
the participants, self-reflexive in how I, the researcher, will both check my power and celebrate my biases, and is contextual in that the students' culture, race, gender, and social standing will be analyzed as part of the research results (Gall, Gall, \& Borg, 2007). I also acknowledge that this type of research is often viewed as hypercritical by other research traditions (Gall, Gall, \& Borg, 2007). Critical cultural research is often considered "subjective," especially when it challenges the dominant cultural view or method (Parker \& Lynn, 2009; Solorzano \& Yosso, 2009).

I acknowledge, nevertheless, that this is dissertation research that requires adherence to academic scrutiny that is often rejected by CRT scholars (Taylor, 2009). Thus, it is important to use a variety of research methods for triangulation and to maintain academic rigor and intensity that is customary in dissertation research. Likewise, Pritchard, Morgan, and Ateljevic (2011) maintain that it is necessary for a critical social researcher to show that she or he is technically trained but reflexive and emotionally competent: "Even to speak of hope and love as academics however makes us vulnerable, as this is associated with weakness, irrationality and emotion — particularly in an academy conditioned to principles of distance, objectivity and rationality. Yet this engages directly with the nature of hopeful tourism knowledge, researcher position, voice, training and control” (p. 951). Therefore, I used grounded theory in my approach to data analysis and the generation of research codes and themes that arise as the data were collected, which is a well-known method for analyzing and reporting research within qualitative methodology (Marshall \& Rossman, 2011). Furthermore, grounded theory data collection and analysis allowed me to retain the flexibility and emerging 
format that is subjective and reflexive, which is required by the critical social ontology of this study, but still maintain the academic rigor that is necessary for dissertation research (Clark \& Cresswell, 2010).

\section{Role of the Researcher}

As mentioned above in the Entrance section, I had relatively easy opportunity gaining access to the site because my colleague, Dr. Green, had already obtained permission for me to observe the classes at LU, observe and participate in the cultural excursions, and had explained this project to his students. Additionally, formal permission in writing from both Portland State University and PSU was sought out by their institutional review boards to carry out research on human subjects. Furthermore, each of the participants was asked to sign a letter of consent that informed them that participation was voluntary and that they were allowed to exit the study, if desired, at any point during the study. In addition, consistent member checks were carried out wherein the participants were asked to verify that the transcripts of the interviews and the observations were accurate. The participants were also allowed and encouraged to review the themes and patterns that emerged during the analysis part of the study for authenticity. The researcher purchased a small gift to a Spanish department store for each of the participants as a way of showing gratitude for their participation as well (approximately $\$ 10$ gift card).

As the researcher, I straddled the line between what Clark and Cresswell (2010) call a nonparticipant observer (an observer who sits in the back of the setting and observes) in the classes at the university, and a participant observer (an observer who 
actively participates in the setting) during cultural excursions. Gall, Gall, and Borg (2007) call this type of observation the participant-observer role in that "the researcher observes and interacts closely enough with individuals to establish a meaningful identity with their group; however, the researcher does not engage in activities that are at the core of the group's identity" (p. 277). Another description of this role of the researcher is what Vogt, Gardner, and Haeffele (2012) call overt participant research. In essence, the study-abroad students knew that I was there to study their experience. Furthermore, I noted during analysis that over the course of four weeks my role became increasingly participant and decreasingly non-participant. Most importantly, I did not hide my role as a researcher at any time.

\section{Exit Strategy}

Often, qualitative researchers expend much energy negotiating access to participants, but fail to develop an exit strategy (Marshall \& Rossman, 2011). It was important for the research participants to know how their information was going to be used and what to expect from the extensive interviews and observations. The studyabroad program had a built-in closing social where the students filled out the post-study questionnaire. During this social, Dr. Green led a discussion on all of the amazing experiences the students participated in over the four-week, study-abroad program. This allowed participants to follow up and express any of their feelings of participating in the study-abroad program. I also reminded them that I would be contacting them in November to follow up on their experience and to provide a final member check before writing up the final research report. 
After the final member-check/interview, several of the participants invited me to be their "friend" on Facebook. I told each of them that I felt it would be most ethical to wait until after the dissertation was defended. However, understanding the high level of collaboration and reciprocity required of the participants in this dissertation, I will accept the "friend" requests of any of the research participants. Furthermore, all of the students, except Kristene, already "follow" my Flickr photos and I "follow" theirs, as was explained in the Photo Analysis subsection above.

\section{Data Management}

In order to ensure the privacy of each participant, it is necessary to secure data to protect the confidentiality of the participants as much as possible. Gall, Gall, and Borg (2007) state, "Once research data have been collected, the researcher must ensure that no unauthorized persons have access to them, and that the privacy of individuals to whom the data apply is protected" (p. 85). Both my phone and computer require passwords to access the desktops. As a further measure of precaution, the Evernote application (where I stored my data) had a redundant password protection (meaning it required another password to get into the file where the data were stored). The files were also encrypted, making it difficult for hackers to access the data, further protecting the confidentiality of the participants.

The interviews were recorded on two different digital voice recorders (mobile phone and digital voice recorder) and the mp3 files of those interviews were stored on both my computer and the cloud-based storage system Google Drive (both require redundant passwords and encrypted files as described above). The voice recorders were 
kept in a locked pocket (TSA-style lock) in my backpack. All participants were referred to as "Participant 1, 2, 3, etc." in the digital files and in the transcripts. When I transitioned to writing about the students in the research report, I changed to the use of pseudonyms, to create a better reading experience.

The dual-format field notes (and any other physical copies of data) were kept in a private journal that was stored in the TSA-style locked backpack pocket. Almost daily, I uploaded pictures of any physical paper copies to Evernote and then destroyed the paper copies. The participants were likewise be referred to as "Participant 1, 2, 3, or 4" in these notes in order to ensure privacy and to protect the confidentiality of the participants. If the participants chose to share any of their reflective journal entries (Kristene shared her whole journal and Kylie and "Kynzee" shared their blogs with me), all names were deleted or etched out and were replaced with their participant number).

\section{Data Analysis}

Mezirow's initial studies were based on grounded-theory data analysis of women returning to higher education (Baumgartner, 2012; Merriam \& Kim, 2012; Mezirow, 2012).

In addition, grounded theory is especially useful to generate new theories or new dimensions of a theory by deriving constructs that explain processes, actions, or interactions among people from the immediate data collected by the researcher during a study (Clark \& Cresswell, 2010; Gall, Gall, \& Borg, 2007). Although TLT has been around for more than 50 years, few studies exist that empirically explore travel for transformation (Morgan, 2010). Even fewer studies exist that look at travel for 
transformation through a socio-cultural lens (Pritchard, Morgan, \& Ateljevic, 2011). This study represents the first study that specifically examined how travel for transformation might be utilized as a pedagogy of the privileged (Curry-Stevens, 2007) through the CRT lens of Whiteness.

The term grounded theory has become so popular that it is often used to mean any type of qualitative analysis (Vogt, Gardner, \& Haeffele, 2012). Therefore, it is imperative to understand what exactly is meant by "grounded theory" when using this methodology as a data analysis tool. Considered to be the theorist to originate grounded theory, Glaser (2012) states "all is (sic) data" (p. 28). He continues:

What does it mean? It means exactly what is going on in the research scene is the data, whatever the source, whether interview, observations, documents, in whatever combination. It is not only what is being told, how it is being told and the conditions of its being told, but also all the data surrounding what is being told. (p. 28)

To collect data for this project, I borrowed heavily from the idea that "all is (sic) data." Anything observed, heard, felt during the research project by me or the participants could be collected, coded, and analyzed. However, Glaser's article has an undertone of a positivist belief in cold objectivity and researcher distance from the data, which was not useful for this research. Furthermore, Glaser argues that researcher and participant subjectivity is something that must be countered in qualitative research, which ran contrary to both CRT and critical social paradigms on which this study hinged. 
Charmaz (2003), however, contends that most grounded theorists write as if their data are objective and proposes an alternative for critical social scholars called "constructivist grounded theory" (p. 250). According to Charmaz (2003), constructivist grounded theory "assumes the relativism of multiple social realities, recognizes the mutual creation of knowledge by the viewer and the viewed, and aims toward interpretive understandings of the subjects' meanings" (p. 250). Constructivist grounded theory specify analytic strategies, not data collection methods; however, researchers are encouraged to collect from varied sources and methods in order to obtain extensive amounts of rich data with thick description.

Although not clearly labeled constructivist grounded theory, this style of data description closely parallels Pritchard, Morgan, and Ateljevic's (2011) study of travel for transformation. They assert that travel for transformation should be emancipatory, social action-oriented, participant-driven, reflective, dialectical, pluralist, and multidimensional. They claim that both the researcher and the research participants should generate themes data analysis through participative storying. They call the researcher a "transformative advocate, with a self-reflexive, passionate, positioned voice, foregrounding the emotional dynamics of research relations" (p. 952).

Under a constructivist grounded theory model, the researcher uses knowledge gained from the literature review to inform decisions, but is open to and reflexive about new findings that are generated during the collection of data. Johnson (2003) captures this reflexivity perfectly: "Initially, ...I tried to approach it inductively through a grounded theory framework. But this missed so much of the nuance of what I had 
experienced that I finally discarded my first draft and let the material itself show me how it wanted to be presented. The result was an autobiographical account of my encounter" (p. 240) with the participants and the research questions.

In order to analyze the data collected through this research project using a constructivist grounded theory method, I divided the analysis into six phases: (1) organizing data, (2) immersion in data, (3) generating categories and themes, (4) coding data, (5) searching for alternative understandings, and (6) writing the report (Marshall \& Rossman, 2011, p. 209).

Organizing the data. As described above, grounded theory requires the researcher to collect extensive amounts of rich data combined with thick description. Marshall and Rossman (2011) stated, "In the early stages of qualitative inquiry, the researcher may enter the setting with broad areas of interest but without predetermined categories or strict observational checklists" (p. 139). Therefore, the key at this point of grounded theory analysis was to collect and organize as much data as possible. I acknowledged that not all of these data would be used in the final analysis. However, the data all served as contextual clues to the themes and patterns that the participants and I caused to emerge, as the data were later coded and categorized. Furthermore, it was imperative to note that while collecting, initial themes and patterns emerged from the researcher and the participants and could serve as topics for interviews and further observations (Clark \& Creswell, 2010). Brown (2009) found that these early themes and patterns that the participants caused to emerge helped collect better data for future interviews. This dissertation research aligns with Brown's findings. 
For example, the photos posted on Flickr served as excellent prompts for the both the formal and informal interviews carried out in this research.

In order to collect, store, and organize data, I used the Evernote app on my computer and phone. Even if I wrote notes in a field notebook, I later uploaded picture images of the notebook into the Evernote app for storage and organization of data (the pages of the field notebook were then destroyed to maintain the privacy of the participants as indicated above). One benefit of the Evernote app is that it is a cloudbased application, which synced notes to both my computer and my phone. This allowed all data to be stored in a single application, which was useful later when I gathered and organized the data for coding. Additionally, the cloud-based nature of Evernote backed up my data in case my phone and computer were stolen. Furthermore, Evernote has excellent searchability, allowing the user to search information through the titles, tags, or text of the application. This was an invaluable resource as codes and themes were developed to organize thoughts, impressions, and ideas for further study. Evernote also lets one highlight, draw on, underline, italicize, and bold the notes, pictures, text, memos, etc. through a companion application called Skitch. One final benefit to Evernote is that it is capable of inserting voice memos, text, pictures, web pages, and emails into the "notes," allowing all to be data as mentioned above (Glaser, 2012). The variety of input methods and the capacity to sync between devices made Evernote a valuable resource for data collection for this research.

Preparing data. Following the collection of data, I gathered and organized all interviews, life histories, field notes, photos and videos, and "all is data" that I had 
collected during the month in Spain into an easy-to-read format in the Evernote application (Marshall \& Rossman, 2011). Part of this collection of materials included transcribing recorded interviews and translating Spanish interviews into English (my undergraduate degree is in Spanish translation), (Clark \& Cresswell, 2010). However, all of the students felt more comfortable speaking in English, so the relatively minor amount of translation (almost entirely from lass observations) had little impact on data collection or analysis. Nevertheless, to avoid any bias of translation, and to ensure accuracy of what the participants said (they all had limited Spanish proficiency), I conducted frequent member-checks with the participants, the LU professors and staff, Dr. Green, and the various tour guides at the cultural excursions.

During my master's thesis I found it easier to mark up the data in a printout version. However, in the six years that have passed since completion of my thesis, it has become much easier to annotate data on a computer. One of the benefits of organizing data on a computer, especially in the Evernote application, is the capacity to annotate, mark, unmark, or annotate items differently if an alternative theme or pattern emerges during analysis using Skitch. 
Immersion in the data. Marshall and Rossman (2011) state that there is no substitute for intimate engagement with your data when doing grounded theory analysis. They further assert, "Researchers should think of data as something to cuddle up with, embrace, and get to know better. Reading, rereading, and reading through the data once more force the researcher to become intimate with the material" (Marshall \& Rossman, 2011, p. 210). Brown (2009) notes that before coding she had to spend weeks repeatedly listening to interview tapes, reading transcripts, reading field notes, noting certain phrases that repeatedly appeared, and noting certain actions or activities that consistently occurred throughout the collection of field notes. Brown (2009) also sought the assistance of a critical friend during this stage to search for alternative possible initial themes.

Upon returning home from Spain, I also immersed myself in the data for several months, spending hundreds of hours listening/re-listening to interviews, reading/rereading field notes, going over observations, and looking at all of the photographs. I marked up initial themes and used the assistance of two critical scholarly colleagues to search for alternative understandings.

Exploring and coding data. After data were collected, it was necessary to develop a coding system for analyzing the data (Gall, Gall, \& Borg, 2007). Grounded theory is a systematic procedure to generate a general explanation that explains a process (Clark \& Cresswell, 2010). Gall, Gall, and Borg (2007) maintain that the researcher collects data into a manageable system to analyze, develops a category-coding procedure, 
codes the data, interprets the results, and then checks for alternative interpretations through peer review and member checks.

One benefit of constructivist grounded theory for the diverse data collection methods I utilized for this research is that grounded theory data analysis was relatively similar to use for photos, transcriptions, surveys, personal biographies, or observations (Gall, Gall, \& Borg, 2007). Clover (2007), for example found that grounded theory was especially useful to match up themes generated by cross examining what participants said in interviews with what patterns manifest in the subjects of their photographs.

Following the collection of the notes and interviews, I carefully read the data searching for salient themes, patterns, or other relevant information. Special attention was paid to data as applicable to three research questions: What elements of transformative learning, if any, did eight students experience during a month-long studyabroad experience in Spain? What effect did the study-abroad experience itself (including second language learning) play in the transformative learning of each student? What role did each student's racial, economic, gender, and cultural privilege (or lack thereof) take part in their worldview/transformations during the study-abroad program?

Coding data is the formal representation of analytic thinking (Marshall \& Rossman, 2011). Codes were developed by matching information from the research questions with information in the data. I then applied a coding scheme to those categories and themes and diligently and thoroughly mark passages in the data using the codes (Marshall \& Rossman, 2011). When generating a grounded theory, I started by underlining the different codes in Skitch for Evernote. I used a different color to code for 
each of the research questions. In addition, I kept a few extra colors available for new codes that appeared while the data were analyzed. As codes turned into patterns and themes, I started to highlight (matching the color of the corresponding underlining code) to highlight patterns and themes that the participants and I cause to emerge from the data. Furthermore, I wrote memos to myself in the margins of the "paper" in Evernote to note patterns among the data. As mentioned earlier, I did all of this in the Evernote application on my phone/computer because it is possible to keep all of the data together in one place and to unmark items if new themes emerge.

Developing description/analytic memos. After codes have turned into patterns and patterns into overarching themes by analyzing data, it becomes essential to start to generate and describe a grounded theory (Clark \& Cresswell, 2010). Likewise, Marshall and Rossman (2011) maintain:

As categories and themes are developed and coding is well under way, the researcher begins a process whereby she offers integrative interpretations of what she has learned. Often referred to as "telling the story," interpretation brings meaning and coherence to the themes, patterns, and categories, developing linkages and a story line that makes sense and is engaging to read. p. 219 I began to "tell the story" by writing notes, reflective memos, thoughts, and insights as I analyzed the data, even during research in the field to generate formal and informal interviews/member checks with the participants (Marshall \& Rossman, 2011). Memos showed how the original literature review, the research questions, and the data collection/analysis created clusters of patterns and themes that eventually came together 
like a patchwork quilt to create the results section of the final research report. Johnson (2003) found this part of data analysis to resemble an autobiography of collecting and coding the data. Likewise, I found that my interaction with the data created a reflection on my interactions, first with the participants, and later, with the data surrounding this study-abroad program.

Validating finding/searching for alternative understandings. After developing and describing a grounded theory, I re-read several times searching for deeper understanding and alternative analyses (Clark \& Cresswell, 2010). Marshall and Rossman (2011) state at this point the researcher "looks suspiciously at his own observations, asking where he might have applied his own biases and interpretations instead of those generated from the actual behaviors, interactions, words, and sentiments of his participants" (Marshall \& Rossman, 2011, p. 220). At this point, I also sought alternative understandings by having at least two seasoned qualitative researchers review my findings, data analysis, and results of the project.

The final chance to search for alternate understandings was with the participants themselves through the final member check/phone interview. I sent a summary sheet to each of the participants that included themes that emerged from their experience as an individual and as a group. It also contained a summary of key moments or interviews with the participant. Furthermore, if anything arose during analysis about which I desired further information, I included it in the summary sheet as well. During this phone interview, I also conducted a follow-up interview about "reverse culture shock," asking the participants what it was like to return home with new understandings. As a researcher 
using a constructivist grounded theory method and a critical social ontology, I deeply valued the participants' insights about the themes that emerged throughout the research project.

Ultimately, however, I understand that the researcher must make final analysis realizing that this is based in her or his own biases/worldview. Therefore, I checked findings with colleagues, utilized multiple data collection methods/sources, spent extended time in field, conducted member checks, analyzed through triangulation of sources, and used peer debriefing to search for alternate understandings (Clark \& Cresswell, 2010).

Triangulation of data (discussed below in the Trustworthiness section) was accomplished by comparing the dual-font notes, open-ended survey prompts, photos/videos, biographies, and interviews, and myriad secondary data sources. Salient themes, patterns, and relevant information will be described during the Results section of the project below. I will then explain and explore the themes, patterns, and relevant information during the Discussion section of the project.

\section{Trustworthiness}

Trustworthiness is qualitative research's conceptual companion to validity in quantitative research (Vogt, Gardner, \& Haeffelle, 2012). Building trust with research participants, collecting and analyzing multiple data points through triangulation of data, and ensuring researcher confirmability and dependability are often ways that qualitative researchers show that their project will stand up to academic scrutiny and will produce reliable results (Marshall \& Rossman, 2011; Vogt, Gardner, \& Haeffele, 
2012). Therefore, following is a description of how I planned to create a project that contained each of those elements of trustworthiness by building trust, triangulating data sources, manifesting dependability, and establishing confirmability.

Building trust. Building trust is an important aspect in the researcher's quest for trustworthy, reliable data (Marshall and Rossman, 2011). Some researchers feel that it is important for the researcher to be immersed in a setting for long periods of time in order to build up that trust (Guba \& Lincoln, 1985). Trust can be the difference between a reliable and credible collection of data and the committing of errors (Boncana, 2010). In order to gain trust I spent long periods of time immersed in the setting and took on an overt participant observer role. Furthermore, I had no official position of power over the research participants. In order to lessen the perceived threat of power that researchers often hold, I was open about my role as researcher and participated in the setting as much is acceptable to build the trust of the participants. Additionally, Liable (2000) asserts that it is important to study people that the researcher claims as his or her own in order to avoid marginalizing the studied population. Studying one's "own people" also allows for a more nuanced and trustworthy data set. For this reason, I chose to study participants with whom I share similar socio-economic, racial, and national backgrounds. Furthermore, by allowing the participants to withdraw at any time and keeping their information private, I also expected that the participants felt free to honestly express their beliefs regarding the research questions. 
Triangulation. Another aspect to show that a research project contains trustworthy information is through the triangulation of data (Clark \& Cresswell, 2010). Triangulation is using multiple sources of data collection, data analysis, data collection methods, multiple participants, and research perspectives/theories to corroborate the validity of qualitative research findings (Gall, Gall, \& Borg, 2007). According to Marshall and Rossman (2012), "triangulation is not so much about getting the 'truth' but rather about finding the multiple perspectives for knowing the social world" (p. 254).

In order to provide triangulation, I collected field notes, photos, and videos, conducted interviews, and reviewed the data collected in order to obtain multiple sources of information so that triangulation of data was possible. Conducting multiple member checks, both during data collection and after the study-abroad program, further enhanced not only the trustworthiness of the data, but helped me to consistently check my biases. I also sought alternative understandings by having at least two seasoned qualitative researchers review my findings, data analysis, and results throughout the project. Moreover, as a dissertation project, a committee of at least four scholars in social science fields reviewed and approved the findings. 
TRAVEL FOR TRANSFORMATION

Dependability. Research involving human subjects involves constantly changing conditions and variables that cannot always be anticipated by the researcher. An important aspect in any research, but especially qualitative research, is for the researcher to show that she or he is prepared to account for changing conditions that will occur as the research is carried out (Marshall \& Rossman, 2011). This is quite a different notion from the positivist, quantitative assumptions of reliability where researchers believe that if a research project has been developed with enough rigor, it should be able to be repeated and replicate the same results (Gall, Gall, \& Borg, 2007; Kezar, Carducci, \& Contreras-McGavin, 2006). However, qualitative researchers assume that the conditions, participants, and the researcher are constantly changing, thereby causing research to be constructed in the particular setting, with that particular group of people, in a fixed moment in time (Lincoln \& Guba, 2003). Therefore, the researcher under this paradigm, must be prepared to accept change that comes with social science research: "This assumption of an unchanging social world is in direct contrast with the qualitative/interpretative assumption that the social world is always being constructed and the concept of replication is itself problematic" (Marshall \& Rossman, 2011, p. 253).

One of the main reasons that I selected grounded theory to use as a data collection method is because I wanted to be open and flexible enough to accept outcomes different from the ones my biases thought might happen (I will speak to these in both the Findings and Discussion chapters below.). Furthermore, I grounded myself in academic literature pertaining to the purposes of transformative learning, CRT, and travel for transformation. If, however, other understandings came of the data analysis, I was open 
to reviewing the literature regarding those outcomes and enhancing the literature review on that finding. Originally, grounded theory research was done this way, where the data were collected before the literature review was written (Charmaz, 2003). Moreover, one of the main characteristics of transformative learning theory is permeability, so it is essential that I am open to alternative understandings, especially when they contradicted my own, in order to model understanding of the phenomenon I am attempting to observe (hooks, 1994; Mezirow, 2012). I understand that the participants would show differing degrees of transformation, if any, which I was not only open to, but I believed that differing reactions to the study-abroad program were precisely what makes this research exciting. In addition, having the study-abroad students consistently review the data being collected and discussing the meanings encouraged them to be co-creators of the initial themes that were generated during the collection of data.

Confirmability. Although qualitative research does not assume the cold objectivity of the research such as in positivist traditions of quantitative research methodology, ensuring confirmability is key to a qualitative research project. Confirmability shows that logical inferences and interpretations were made by the researcher from the collection, analysis, and findings of data (Marshall \& Rossman, 2011). In order to collect confirmable results, I attempted to show the logical manner in which the methodology was created and rooted in the research questions and purposes of this research project.

Understanding that I have subjectivities and biases as a researcher, I put into place protocol for managing biases such as triangulation of multiple data points, cross-checking 
the data points, peer review, and consistent and repeated member checks. I also selected a wide range of data collection methods from multiple sources to ensure the collection of rich data. It is important to note that the purpose of this research was not generalizability of findings, but rather an in-depth, rich, and co-storied description of the participants' views of their study-abroad experience. My understanding of CRT, TLT, and transformation for travel increased the likelihood of a complex and nuanced description rather than diminished it. Furthermore, I asked Dr. Green (who attended all of the cultural excursions and many of the class sessions) to be a critical cross-checker and peer-reviewer of the data collected and the inferences made in the initial generation of themes produced while data were collected and interviews were used to check emerging themes and patterns. To ensure privacy of the participants, however, we spoke in vague terms so that I did not betray the trust that the participants had that I would guard their confidentiality.

For example, in the field I noticed that none of the participants brought computers to the study-abroad program. Originally, I thought that this might be an indication that they did not take their studies seriously. It confused me, because most of the participants seemed like stellar students from my observations. Rather than naming the participants, I asked Dr. Green, "I have noticed that many of the students did not bring a computer, do you have any thoughts about this?" Dr. Green and I could cross check this information without betraying the individual participants' confidentiality. In this instance, I found out that Dr. Green told them specifically not to bring a laptop, because he feared it would make travel more difficult and because he feared their laptops getting stolen. Levante 
TRAVEL FOR TRANSFORMATION

University (and some of the host families) had computers and Internet access that the students could utilize. When I found out that Dr. Green asked them to leave their laptops at home, it changed my narrative. Following up with the individual participants during member checks confirmed Dr. Green's hypothesis.

\section{Ethical Considerations}

Research on human subjects always requires careful deliberation of the ethical considerations involved (Marshall \& Rossman, 2011). In order to maintain ethics in research procedure, I chose to do research on adults of sound mental condition. In addition, I asked each of the participants to sign an informed consent form that explained the purpose and limited associated risks with participating in the study. Furthermore, the participants understood the methods of secure data management and personal privacy outlined in the data management section. The participants were also allowed to withdraw from the study at any time, for any reason. Additionally, the researcher sought the approval of the Institutional Review Boards (IRB) of both the Portland State University and Plain State University, thereby acquiring the oversight of those organizations. I strictly adhered to any stipulations provided by either of those IRBs.

Researchers especially have ethical duties to protect the privacy of participants themselves, as much as is possible (Vogt, Gardner, \& Haeffele, 2012). The two parts to privacy are anonymity (researcher does not know the identity of the participant) and confidentiality (researcher does everything possible to mask the identity of the participants). The data collection methods of this research project rendered it impossible to maintain the anonymity of the participants because I was with them six to twelve hours 
a day for a month and performed in-depth interviews about their lives. This made maintaining their privacy through confidentiality essential. Therefore, I was meticulous to be sensitive to confidentiality in the way data were collected and presented. Initially, the participants were assigned a number (ex. "Participant 1"). As I began to write the findings, pseudonyms replaced their numbers in data collection and presentation. The participants were also told to help each other maintain each other's privacy, especially from the focus group interviews, where they knew what each other said. Moreover, I omitted background information and used methods of concealment about PSU and LU to further protect the identity of the participants.

Finally, another important ethical decision of researchers is to make sure that the dissemination of findings does not harm the participants (Tuck \& Yang, 2014). Therefore, it was important for me to verify that the potential benefit of the research outweighed potential harm, that the participants were free to end the experiment at any point (including withdrawing their data from the analysis), and that I should stop if I realize that it was causing harm to the participants (Vogt, Gardner, \& Haeffele, 2012). Knowing that transformative learning often requires one to search her or his pasts for understanding, I was especially sensitive to the participant's reactions during interviews to protect them from the harm by probing issues that cause them to re-live pain or discomfort. Furthermore, the letter of consent and my overt role as researcher informed the participants of what my research was and how I planned to use it. Also, as stated previously, the use of member checks also ensured that how the data are disseminated was understood and acceptable to each participant. Moreover, the 
participants were co-generators of knowledge, codes, and themes in this study, which gave them voice over how their transformative study-abroad experience was told (Pritchard, Morgan, \& Ateljevic, 2011). Finally, I tried to prepare myself to respect diverse views, especially when they contradicted my own (this came into play several times throughout data collections and will be discussed in the Discussion chapter). The essence of this final paragraph is to let the reader know that the emotional, physical, and mental well-being of the participants were (and are) more important to me than my dissertation study. 


\section{Chapter Four: Findings}

The purpose of this chapter is to organize and summarize relevant findings about the transformative experiences (or lack thereof) of eight students from a medium-sized, Midwestern university as they went to a study-abroad program in Spain. Because I used constructivist grounded theory data analysis, it was necessary to use plentiful direct quotes from the participants and highlight their experiences as "co-participative storiers" (Pritchard, Morgan, \& Ateljevic's, 2011). As a researcher, I was (and continue to be) humbled and amazed at the participants' gifts of time, emotional energy, and personal transparency. Moreover, because constructivist grounded theory "assumes the relativism of multiple social realities, recognizes the mutual creation of knowledge by the viewer and the viewed, and aims toward interpretive understandings of the subjects' meanings" (Charmaz, 2003, p. 250), it was essential for me as the researcher to add my interpretations of observations, interviews, and experiences with the participants. Together, our understandings join to provide triangulation of data sources and to enrich our co-constructed reality (Gall, Gall, \& Borg, 2007; O'Sullivan, 2012). Therefore, my experiences and expertise of critical-social TLT, CRT, and travel for transformation will be included in the findings connecting their voices through narrative explanations, analysis, and thick description of the extensive data I collected during the study-abroad program. Similar to Pritchard, Morgan, and Ateljevic (2011), both the researcher and the research participants generated themes and I considered myself a "transformative advocate, with a self-reflexive, passionate, positioned voice, foregrounding the emotional dynamics of research relations" (p. 952). Through this co-participative endeavor, deep, 
rich, and complex understandings emerged that reflected the perspective of the students involved in the study-abroad program in order to delineate principles of how adults learn and transform toward more permeable worldviews

In order to analyze the data collected through this research project using a constructivist grounded theory method, I divided the analysis into six phases: (1) organizing data, (2) immersion in data, (3) generating categories and themes, (4) coding data, (5) searching for alternative understandings, and (6) writing the report (Marshall \& Rossman, 2011, p. 209). Phases one through four took approximately six months as I sought to understand and organize the data, generating categories, codes, and themes. As I wrote the findings for each participant, I had two experienced researchers review the findings and challenge me to search for further understanding. Although I used knowledge gained from the literature review to inform analytical decisions, I remained open to and reflexive about new findings that were generated during the collection of data. For several of the participants, these new findings appear with the term other in the subheading.

\section{Purpose}

The purpose of this project was to describe what elements of the transformative learning process students who participated in a month-long study-abroad program in Spain experienced. In addition, I explored what the experience of traveling to an "other" place and experiencing cultural and linguistic "otherness" had on transformative learning (Johnson-Bailey and Alfred, 2006; Morgan, 2010). Moreover, I examined transformative 
learning through the critical race theory lens of Whiteness to analyze what role racial and cultural privilege (or lack thereof) played in travel for transformation.

\section{Research Questions}

In order to achieve the purpose above, the research in this project was guided by the following questions:

1. To what extent did eight students experience the elements of transformative learning during a month-long, study-abroad experience in Spain?

2. What role did encounters with cultural and linguistic "otherness" play in the transformative learning (or lack thereof) of each participant?

3. What was each student's racial, economic, gender, and cultural identity? How did each student's identity impact her or his worldview and transformations (or lack thereof) during the month-long, study-abroad experience?

\section{Overview}

All of the participants in this study exhibited some elements of transformative learning during the study-abroad program. Additionally, all of them experienced either culture shocks or what we came to label "cultural surprises" (the students felt culture shock seemed negative), which closely parallel Mezirow's (2012) cognitive dissonance. Many of the participants also experienced transformations. The purpose of this chapter is to highlight the experiences of each participant through the lens of the research questions.

Because social class emerged as a repeated theme during analysis, I have chosen to place the students in perceived (and stated) order from highest to lowest socioeconomic class. Class data were mostly arrived at through the participants' self- 
descriptions. However, in a couple of instances, analysis of the participants' words and actions caused me to either raise or lower their placement in the group of students. In addition to class distinctions, gender, race, cultural identity, and the hometown census data played a role in distinguishing the students. Due to the length of this chapter, I feel that this ordering makes this chapter easier to read and understand the socio-cultural variables at play in the exchanges among the students for the reader.

\section{Participant One-“Kristene"-Independence}

As mentioned previously in the Methods chapter, I first met Kristene during the opening orientation meeting on the Saturday night before the Levante University (LU) classes began. I explained my research to the students. All of the students, including Kristene, quickly signed my letter of consent and I sat back down and observed the rest of the meeting. One of my fears early on was to mistake a name and write down information about the wrong participant. During data collection, I was particularly concerned that I would mistake the numbered coding system (participant one, participant 2 , etc.), and then collect data on the wrong participant. I also did not want to speak in generalities about the group of students until I got to know them personally, which would cause me to lose individual data for the first few days of the study-abroad program. Therefore, I resorted to a memory trick I have used in my teaching. I took a picture of each of the students, saved the picture to Evernote, put the participant's name as the title of the note, and then labeled their picture with a number (ex. "1"). This number became their participant number, helped me remember their names, and helped ensure that I was collecting data about the correct participant. I felt like the confidentiality of the students 
was protected because it was in a password-protected device and file. Also, I felt that by simply putting a number, rather than labeling the photo "participant one," anyone who might access the individual file would not realize what the number meant.

Therefore, I asked each of the participants if I could take their pictures to remember their names. All of them happily agreed, until I got to Kristene. She quickly and curtly responded, "No." I told her that was perfectly acceptable and reminded her that she did not have to participate in the research and could withdraw from this or any other part of the research design because her participation was voluntary. As mentioned in the Methods chapter, over the first few days of the study-abroad program, every time I mentioned the Flickr data collection method, Kristene made a negative face and the other participants would laugh. I wrote in my field notes, "I am afraid Kristene will derail the Flickr data collection method." I also noted, "I really think Kristene is a major key to entrance with the group. However, every attempt I make to get to know her, I am met with even more resistance and hesitation from her." Dr. Green observed the phenomenon one day and encouraged me to discuss with her again that she could opt out of this or any other part of the study. She told me that she did not believe that she was photogenic. When I reminded her rights to participate or withdraw from this part (or any part) of the study, she said that if I would do my best to not look at pictures of her and would tolerate her not participating in the Flickr data collection, she would became a happy ally to the study.

After this conversation, Kristene was an invaluable asset to data collection. As previously mentioned, I believe that although Dr. Green was the LU institutional 
gatekeeper for this research, Kristene was the gatekeeper to acceptance with the student participants. She was often the first to volunteer for individual interviews. She even offered to send me her journal after the study-abroad program to make up for the discrepancy in data. This journal was 89 pages (hand written) long, which more than compensated for the lack of photos of or by her. Two other participants (Kylie and Kynzee) heard her offering me her journal and invited me to their blogs that they were writing of the study-abroad program. Tess also offered her journal to me after hearing what these three did, but never followed through, despite two reminders.

On the first day of classes I noted that Kristene's Spanish was the best out of the group of students (Tess, discussed later, was a heritage speaker, but intermingled Spanish and English to such a degree that she was often unintelligible $)^{4}$. During the entrance interview on the way to Granada ${ }^{5}$, she informed me that she had studied Spanish for seven years in high school and college previous to the study-abroad program. I noted in my field notes several times that Kristene was an excellent student. She knew how to participate enough to let the professors know she was engaged, prepared excellent

${ }^{4}$ Dr. Green, who is certified as an Oral Proficiency Interview Tester by the American Council on the Teaching of Foreign Languages (ACTFL) supports my observations about Kristene's (and other students') Spanish. As part of his classes at PSU, he gives oral proficiency interviews with the students regularly. The only caveat he offered was that for the interviews to be "official" according to ACTFL, he cannot know the students personally. However, he said that my observations concur with his observations from performing multiple interviews with the participants. Additionally, although not certified, I hold a bachelor's degree in Spanish translation and interpretation.

${ }^{5}$ To maintain consistency of reporting throughout this chapter, the interview on the way to Granada will be labeled the "entrance interview." The interview during the last week of classes will be labeled the "exit interview." The interview to follow up on my findings, and to ask about reverse culture shock five months after the study-abroad program will be labeled "member-check interview." 
presentations, and did well on tests and other assignments. Additionally, in my field notes I discerned that she often looked around to see if anyone else would answer, and then she would answer. I inferred from her body language and this system of looking around each time the professors asked a question that she knew most of the answers, which she confirmed in interviews. Juan's class was essentially a duplicate of a class she had taken at PSU. She hated art and literature, but really liked Ana as a person and felt bad that the other students did not participate. However, I observed that she would occasionally break from this pattern when she got excited about something. She then controlled the discussion because her Spanish ability allowed her to express herself more clearly and quickly than all of the other students, except Tess (discussed later).

Kristene repeatedly described her hometown as a "boring little suburb" $(93,000$ residents) which was a mostly White (86\%), upper-middle class (median household income $\$ 77,000$ ) town that was 30 miles away from a major United States city (census.gov). She was 20 years old during the study-abroad program and had just completed her junior year of college. She majored in elementary education and had a Spanish minor. Her parents paid for her trip and her dad referred to it as a "trip of a lifetime." Only Kristene and Tess stayed after the study-abroad program to do further travels. Her post-Levante travels included Barcelona, several cities in Italy, Paris, and London.

When I asked in the entrance interview why she decided to come to Spain, she said: 
"I had been studying Spanish for seven years now, and had never really met a native speaker or gotten a chance to use it outside of class, and so, once I hit college and realized what I really wanted to do, I almost needed to be fluent, or, more fluent than I was... So this was kind of the perfect opportunity, and I got to see the country that I always wanted to see."

When I asked her why she came to Spain rather than some other Spanish speaking country she stated, "It was always Spain, it was never South America or Central America, it was always Spain. I really don't know why, just always was.”

Elements of TLT Kristene exhibited during study-abroad program. Kristene exhibited multiple experiences with culture shock and cognitive dissonance during the study-abroad program. She also experienced both an incremental transformation as well as an epochal transformation. I will also discuss Kristene's expectation to try as many new things as possible, while on this study-abroad experience, which matches Ross' (2010) explanation of travel placing an individual in a state of "otherness" through a willingness or expectation of novelty. In addition she experienced both cultural and linguistic "othering" in the form of both sizism and linguicism, which align with both Morgan's (2010) findings that cultural and linguistic "otherness" can create cognitive dissonance that leads to transformative learning. Finally, Kristene's biggest transformation of independence can be seen as both transformative learning (Mezirow, 2012) and probable manifestations of both racial and class privilege (Johnson-Bailey, 2012). 
Cognitive dissonance. In the following section, I will discuss culture shocks where Kristene experienced cognitive dissonance. Nudity, food, diversity, and personal space issues where her biggest (and repeated) experiences with cognitive dissonance. The bullfight, sanitation, and other minor examples of cognitive dissonance (which I will label cultural adjustments) are discussed at the end of this section.

Nudity/sexuality. During our entrance interview when I asked what shocks or surprises the first week in Spain brought, Kristene mentioned that the nudity on the beaches, in the art, and in the media was not something to which she was accustomed. After ten days of the study-abroad program, Kristene went to the beach. In her journal she stated, "Still not used to the nudity and toplessness going on, but at least it doesn't affect me [as much]." During the last week, her family took her to a salvaje beach. Salvaje would be typically translated "wild," "untamed," or "wilderness." However, she quickly found out that in this case, to her host family, rather than being "natural," it was a "naturist" beach. She stated, "I saw more penises on this beach in 5 minutes than I've seen in my whole life_-included textbook pictures... I was becoming okay with it until my [host mom] decided to take her top off. That I was completely unprepared for." Her host parents sensed her discomfort and asked her if she wanted to leave, but Kristene convinced them she was okay. In her journal Kristene stated, "On the inside, I would've preferred to leave but that would've only brought questions from [my host siblings], which I definitely didn't want." However, during the exit interview, she mentioned that this was one area where she had become more open-minded. She made it clear that this was something that she felt was okay for others, but not something she would ever take 
part in.

Several times during Ana's Levantino Art and Literature class, Kristene blushed as art was displayed that showed nudity. One time, during class, Ana had the students read a passage about an elegant woman who used the Spanish "z" ([0], unvoiced, interdental fricative, similar to the English "th" in bath). Ana demonstrated the way that this woman's tongue would have gone between her teeth and discussed how the men of the village loved to watch her talk so they could watch her tongue go in and out of the woman's teeth. Ana picked up very quickly that Kristene and Tess (discussed later) had the best comprehension of the group and would often call on Kristene for reactions. When she asked Kristene what she thought of the passage, her body language indicated timidness and she stated that she did not know how to explain it. When Ana pushed her to try, Kristene said, "Sexual," and she blushed.

On the last day of classes, Kristene told me how she was packing to leave on her trip around Europe and her tampons were on her bed. Her eight-year-old host brother came in and asked what they were. Kristene would not answer so the host brother went in and asked his mom. Kristene was shocked that the host mom said, "You know... you have seen me use them" and then made a gesture like she was inserting the tampon. Kristene was shocked and said she would never tell an 8 year old what a tampon was used for. She said she doesn't even think her 16-year-old brother understood what they are. Spanish openness to the human body, nudity, and sexuality was a culture shock that several of the female participants, not solely Kristene experienced.

Besitos and personal space. Kristene never appeared to become comfortable with 
the customs of personal space and besitos that are common ways of greeting and departing in Spain. When women meet, greet, or depart from both men and women in Spain, it is customary for the woman to tough right cheek to right cheek and make a kissing sound, followed by touching left cheek to left cheek followed by a kissing sound. Men typically shake hands and either grab each other's elbows, forearms, or give an abrazo (hug) with the other hand. In addition, it is customary for people of both sexes to stand much closer when talking than they do in the United States. I noticed that my torso would often be touching the other person's torso when we were talking. I pointed out to Dr. Green after the first week of classes that the participants still did not greet with besitos or abrazos. He explained that he is always shocked/disappointed that it takes his students so long to open up to this tradition, because it adds to Spanish stereotype of Northern Europeans and North Americans as stiff and cold.

Kristene never became comfortable with this cultural tradition. Although this was confirmed in multiple interviews, an experience that happened when the participants went on a cultural excursion to Granada provides an example of Kristene's experiences with besitos. Levante University hired a tour guide named "Manolo" to accompany the participants to and from Granada, as well as to give a walking tour of the city and provide support to the students at the tablao de flamenco. Kristene described Manolo in her journal as "our very gorgeous tour guide," yet she found it foolish that the other female participants flirted with him. "To get and sit there and watch five girls flirt with one guy is just so sad." On the bus ride back from Granada, several of the female participants, including Kristene, asked me why I, as a married man, "kissed" our Alhambra tour guide 
"Susana" before and after the tour. I informed them that Dr. Green did too, because it is the traditional way to greet and part company in Spain. Several of the participants were shocked. Jennifer (the next participant discussed in this section) said, "You just don't do that!" I informed the participants that it is the equivalent to our handshake in the United States. Tim, the only male participant, said he would happily do the elbow grab with the bus driver and Manolo as we departed. I encouraged the rest of the participants to give besitos a try and they all said they would. As they got off the bus, they all stared at each other and only Tim did the typical Spanish parting gesture. Dr. Green (who enjoyed my description and invitation to participate in Spanish greetings) and I also departed with an abrazo to the bus driver and Manolo. The female participants stood around giggling and apologizing for "chickening out," saying "it is just too weird." Manolo, told them "I will teach you how we say goodbye in Spain." So he walked around giving each of the female participants besitos. They all did, except for Kristene. She was looking down in her bag when Manolo approached. Manolo tried to give her besitos and she turned her head away and looked shocked. He felt like he surprised her and that she did not see it coming (which her journal confirmed). He apologized for catching her off guard and leaned in to give her besitos once more. She refused again, and said "No. No."

As another example, on the last day of classes, Kristene walked up to me and asked if they had to say goodbye to Juan, the Spanish history teacher "the American or the Spanish way." I told her it was her personal space and that she was free to do whatever she chose. She asked, "What do you think Juan is going to do?" I told her that he is Spanish, so he would probably prefer besitos, but that he teaches students from all 
over the world (and was an avid feminist), so he would respect her wishes. I don't know if he overheard our conversation or if he could sense the participants' reticence, so he asked, "Are we going to do this the American or the Spanish way?" Most of the students gave him both abrazos and besitos. Kristene gave Juan besitos, but looked very uncomfortable and stiff.

Bullfight. The day before the students went to the cultural excursion of the bullfight, Juan explained the process during Spanish History class. Kristene was visibly disgusted. I overheard her say, "Is it ok to leave after a little while if I do not like it?" As she imagined, Kristene severely disliked the bullfight. In her exit interview she stated, "I didn't think it was necessary to be killing all of those bulls for entertainment." She also expressed concern for the horses that had blinders on and were used by men who stabbed the bulls with spears to anger/tire the bull. In her journal, she wrote, "I almost puked watching that." Dr. Green allowed any students who were uncomfortable with the bullfight to leave at the intermission. Kristene and several others did. She said in her exit interview, “It's truly terrible and I'm so glad Dr. Green let us leave at the intermission."

Diversity. During our entrance interview, Kristene expressed that she felt that diversity was different in Spain than it was in the United States. She said that in the United States there exist cultures from "all over the world." However, in Spain, she perceived that most of the individuals are from countries geographically adjacent to Spain, even though the individuals are racially, nationally, and linguistically diverse:

I guess I'm surprised by the amount of diversity here, but on the other hand, I was 
kind of surprised by the lack of it. Like, they do have quite a few different cultures and groups and ethnicities here, but I don't know, I guess I was expecting it... it's all from a, the same general area, whereas like in America, it's very allover-the-world. It's not about what's close, it's, you're in America, you could literally be from anywhere in the world, and no one's going to be surprised by it. Water conservation. Kristene, like others, mentioned a dislike for the water conservation habit of not flushing the toilet when someone only urinated. Water was much more expensive and scarce in Spain, especially in Levante. Juan explained during one of the classes on environmentalism in Spain that Levante had no natural sources of water, so the city buys water from another province via an aqueduct system and mixes the tap water with half salt water from the Mediterranean. Kristene wrote in her journal her unwillingness to keep this tradition:

They don't flush their toilets, or at least, $m y$ "family" doesn't, as often as I would like them to, because they don't do it after every time they use the bathroom, which...grosses me out. And like, I walk in there, and...you can clearly tell people have gone to the bathroom four or five times without flushing, and... I can't do it. It's gross. And I know it's a waste of water... Ugh. I can't do it. I know they're so, so conservationists here, but ...that's just one step I can't take I don't think.

Cultural adjustments. Kristene described a myriad of minor observations I would label cultural adjustments rather than culture shocks or cognitive dissonance. They were different than the United States, but did not make her question the world or 
her place in the world (O'Sullivan, 2012). For example, she stated in her entrance interview that she had a difficulty getting used to the Spanish eating schedule (the biggest meal of the day is at 2:00 p.m.): "The strangest thing is getting used to the scheduleeating a huge meal at 2:00 in the afternoon and then getting to take a nap." In her journal she wrote, "It's almost 8:00 at night and it feels like 4:00. Host siblings are just sitting down to do [homework] at 8:00 pm. I love taking a nap, though.” Other examples mentioned in interviews and her journal include getting used to a new meaning of punctuality (Spanish culture shows up on time for school, work, and church, but social events have a much more loose meaning of meeting time compared to the United States); wearing slippers inside the house; more smoking than in the United States; an initial annoyance at the constant ambient noise (cars, car horns, people talking on the streets, dogs barking, construction); and, getting blisters from walking everywhere rather than driving. There was some minor tokenism in her journal when she saw gypsies for the first time, "It was crazy to realize that some of these vendors were gypsies. After all the research I had done, I was beyond thrilled to see some in person." Being from the Midwestern United States, Kristene developed a cultural surprise of a love for the beach. In her journal, she wrote, "I'm excited to park my butt in the sand and do nothing else. The beach is picture perfect and I'm excited to test it out." She spent as much time as possible there and went to several different beaches. Finally, I also perceive from her journal that the classes were harder (especially the tests) than she thought they would be.

Transformations. As stated in the literature review, not all learning—or all travel for transformation — results in transformations of personal paradigms. To be considered 
transformational, an individual must experience deep shifts in perspective, increased awareness of personal paradigms, increased openness to different ideas, a deep structural shift in ways of seeing or acting in the world, a change in understanding power relations or structures of class, gender, and race, or a more autonomous use of personal agency (Cranton \& Taylor, 2012; Ettling, 2006; Mezirow, 2000; O’Sullivan, 2012; Taylor \& Elias, 2012). Mezirow (2012) explained that transformations can be small, incremental changes that happen over time or epochal changes that are "sudden, dramatic, reorienting insights" (p. 86) that challenge/change a person's core identity or worldview.

Transformations invite people to live more deeply, cultivate hope, instill in them a desire to act, and emancipate them from unsustainable attitudes and behaviors (Tisdell, 2012). Both types of transformative learning create a change in, or better understanding of, how one sees and acts in the world (O'Sullivan 1999, 2012). Kristene experienced an incremental transformation in how she sees politics and an epochal transformation in feeling a sense of autonomy and independence like she had never before experienced. Incremental transformation: Politics and open-mindedness. In the exit interview, Kristene and I talked about how much Juan's class directly addressed culture and politics and how Ana's class discussed how art and literature were reactions to the politics of the time period they were created. I asked Kristene, "Has anything you've learned in [the] classes, or experienced, changed how you view society, how you view culture, how you view politics?" She answered that her study-abroad experience in Spain and the classes made her "even more liberal than I already was." She went on to discuss how she appreciated openness to things like topless sunbathers or same-sex couples holding 
hands: "I'm trying to understand why that doesn't happen in the U.S. And, honestly I have no answer for that. But, I would like to see it happen more rapidly than it currently is."

During Ana's class the last week, Kristene delivered another critique of society in the United States. She argued that she thinks people have a lot more stress in the workplace in the United States than people in Spain. Ana explained that in Spain workers really only work for the 40 hours a week, whereas in the United States people are being convinced to work 50-60 hours a week in order to make more money. This was quite a change from the trip to Granada. During the trip to Granada, Kristene and others asked me why the bus driver got a 45-minute break after driving two hours. I explained to them in Spain it is commonly believed that taking care of workers improves safety for everyone. They all felt it was silly to take a 45 -minute break after two hours of driving. However, it appears that this, and several other experiences, must have changed her perception of the stress that workers in Spain experience versus workers in the United States.

Epochal transformation: Independence. Throughout the study-abroad program, I asked the participants to give me "themes" for each week. The overarching themes will be discussed in the Discussion section of chapter five. During the exit interview, I asked Kristene if there she had a theme for the second half of her study-abroad experience. She replied:

I would almost want to say, like, independence. 'Cause we... went from the whole "culture shock, no idea what's going on"... to ... "I am twenty years old, I 
do want to go out, I $d o$ want to actually experience more than the 'inside-thehouse' culture."

From my observations, I would agree that this was Kristene's biggest transformation. During the entrance interview, Kristene explained that it was a long process convincing her parents, especially her dad, that she was going to be safe during a month-long studyabroad program, followed by a two-week travel around Europe. About her mom, she stated, "my mom finally trusted me to send me out of the country. Or, she, not 'trusted me,' but was more comfortable sending me out of the country." About her dad she said, I'm twenty... you have the whole process of convincing the other parent that I was going to be okay, that was fun. My dad wasn't exactly thrilled with it. It wasn't even the money or anything like it... It was, “She's gonna go live 5,000 miles away from home for a month." And then, I'm actually staying afterwards, so that was a little nerve-racking for him, too.

From this and other conversations, I got the impression that Kristene had grown up in a stable, comfortable environment (which she confirmed in the member-check interview). This afforded her many opportunities. For example, as previously mentioned, I wrote in my field notes that she was an excellent student and knew how to appear engaged and prepared for class each day. She also was often a leader who spoke for the group (I could never deduce whether this was her natural personality or her increased capacity to express herself in Spanish). However, I also observed situations where her protective, comfortable upper-middle class upbringing also instilled in her a limited sense of personal autonomy. 
For example, on the morning of the orientation, Dr. Green received a panicked phone call from Kristene. She and several of the other students had arrived in Levante a day prior to the orientation and had spent the night in a hostel. Dr. Green said that they were concerned because they had to check out of the hostel at noon, but the orientation did not start until 6:00 p.m. She asked Dr. Green what she and the others should do. Because I could clearly hear Dr. Green's side of the conversation, I noticed that rather than offer a solution, Dr. Green offered a couple of ideas like, "Well, some hotels will let you keep luggage behind the front desk or some cities have lockers at the bus or train station." Kristene never told him what she did, but she figured it out. I wrote in my field notes that I was impressed that Dr. Green gave the students possible suggestions rather than figuring it out for them.

Dr. Green explained that he feels that an important part of both linguistic and cultural autonomy during study-abroad programs is for students to develop the skills necessary to communicate in a foreign language, navigate a city, and obtain necessities. He told me that he was going to do an experiment during the last day of the Granada cultural excursion. Dr. Green had approximately five hours of free time scheduled for the students to be able to explore the city. He explained in Spanish (he always spoke in Spanish to the students unless their health or general welfare was at risk) that they had five hours to explore and that they were to meet back at the same spot from which they all departed. He asked me not to follow the students because he did not want them to use my Spanish skills or experience in Spain to find a restaurant or to explore. As we walked to go grab something to eat, he explained that he hoped the students would have a 
confidence-building experience. As we sat down to eat, we saw all nine students (the eight participants, plus the student not studied) walk by with Manolo. Kristene wrote in her journal, "Dr. Green and James left us in a city we knew nothing about, after getting everyone really confused about the meeting point, I really regret not making myself write down [the meeting point or the directions about how to get food]." She expressed frustration about that even several other times in conversations with me. However, the following Monday, she wrote in her journal that she was "a lot more comfortable now that she had developed a routine" and mentioned, "I'm pretty positive I'm not going to want to leave."

After two weeks in Spain, Kristene asked her host mom to help her arrange a bus ticket/reserve a hostel so she could fulfill a lifetime desire to go to Madrid. In her journal she described her excitement at travelling by herself to a foreign city she knew very little about, "So today, here I am. In Madrid. It's kind of crazy to think about, but I completely travelled here on my own and got to where I needed to be. I'm feeling really proud of myself right now." She expressed in her journal the confusion of navigating a city she had never been to before, "Got [to Madrid] and ... had no idea where I was... [I] walked the twenty minutes to the hotel only to get to the wrong hostel" Kristene asked for directions and finally arrived at her hostel, which she described as "sufficient for a night's sleep, I guess." She explored the city, which was a huge departure from the Granada excursion one week earlier. She wrote in her journal, "I just kinda wandered around 'cause I didn't really know what part of the city I was in or anything. Turns out I was like 2 seconds from Puerta del Sol. I didn't realize that, how close I was to the city 
center." Kristene also expressed excitement at discovering new modes of public transportation, "Okay, I'm going to be brave, and I'm going to take the metro." She further discussed in her journal her amazement of how the subway system got her where she needed to go and explained a preference for the subway over Levante's bus and tram system.

Following her personal excursion to Madrid, I conducted an interview with her to explore how the trip went. When I asked her why she chose to go to Madrid for her one excursion-free weekend she stated:

I had always wanted to go to Madrid... I wanted to study abroad in Madrid, and then I realized how expensive Madrid was, so that quickly became not an option. And so, when even my host-family was talking about it, they're like, "Oh, you know, it's only two... hours away by train. It's totally an awesome place. You should go." ... I'd always wanted to go, so I said yes, because this is the only weekend I really had. My mom was like, “This is something you've always wanted to do. You're already in the country. You can't just not go."

Kristene's personal cultural excursion to Madrid appeared to be a turning point for her to develop her self-identified sense of independence, confirmed by her journal, multiple interviews, and my observations. Although she loved Madrid, Kristene was happy to be back to the routine of life in Levante. It seemed like she developed a "home" feeling at Levante.

Kristene's journal is full of examples that after her Madrid trip she asked for directions and went in search of new experiences more. For example, she found a bar 
that she (and the other participants) liked, plus tried other ones. Additionally, she searched for other types of food (ex. Mexican) beyond the American/Spanish she wrote about the first couple of weeks. On the last day of the study-abroad program, she wrote in her journal that she is sad to say goodbye to "Jennifer" and sad that Jennifer's adventure was ending before Kristene's two-week travel around Europe.

Following the study-abroad program, she went to Barcelona by herself, and did a lot more walking around and sight/food finding than she did in Madrid. Kristene wrote with fascination about constantly gets hit on by men, which made her uncomfortable, yet she would talk to them to experience the conversation. She met people in hostels, bars, cafés, and newspaper kiosks and had meaningful conversations with them. She increased her experiences with public transportation, travelling by subway, tour bus, gondola, and ferry. She wrote about her concerns going to Rome, where she would experience a new language and culture. Kristene wrote, "I'm hoping for the best and preparing for the worst." By the end of her two weeks, she had experienced six cities in four countries (Spain, Italy, France, and England).

\section{Other transformative learning: Willingness to experience new things. Kristene} valued the idea of doing things/trying things for the first time. Food served as a metaphor for Mezirow's (2012) and Morgan's (2010) concept of willingness. Kristene's journal is replete with information about the new and different foods that she tried (figs, quail's eggs, paella, paella negra, fried tomato sauce on rice, fried mushrooms). Overall, her journal, interviews, discussions I overheard, and answers to questions in the classes indicate she was excited to be trying new food, even if she did not like it. After trying 
hard-boiled quail's eggs she wrote, "I can say I tried it, which is the whole point: experience, and I'm certainly getting a lot of that." I noted in my field notes that when José Luis asks how Spain was different than the United States, Kristene immediately said, "Food." After one week in to the program, she wrote in her journal, "How come Americans don't (or can't) cook like the Spaniards? It's not fair!" Another experience indicative of this personal philosophy of willingness was when she and a few others stayed up all night to watch the sun rise over the Mediterranean. Unfortunately, it was a hazy morning. However, she wrote in her journal, "I can still say I stayed up to watch the sunrise [over the Mediterranean]." She also noted in her journal that she was so sad to miss the fireworks from Kynzee's balcony (her host family lived adjacent to the plaza where the fireworks went off), that she cried because she missed a unique opportunity to experience something new. I believe this attitude of willingness to try and experience new things led her to her biggest transformation—independence. Kristene's willingness to explore new experiences closely parallels Ross' (2010) description of the novelty of travel opening a person to transformative learning.

Other transformative learning: Reflection. Kristene wrote several times in her journal that she valued the time to read and to write in her journal that the study-abroad experience granted her. Mezirow $(1997,1998,2000)$ maintains that critical reflection and literacy are keys to transformation. However, from the writings in her journal, the interviews, and the observations, there is no indication that Kristene's reflections or books were critical in nature. Nevertheless, the time spent reflecting upon her experiences may have given her space to develop cognitive dissonance or 
transformations. As a researcher, the information that her journal added to my analysis was invaluable to seeing her perspectives of the cultural excursions and the classes, and will be a suggestion for future studies.

What role did encounters with cultural and linguistic "otherness" play in the transformative learning (or lack thereof) of each participant? As stated in the literature review, travel uniquely opens a person to transformative learning because it takes an individual out of daily routines and positions the learner into a state of “otherness," both geographically and culturally (Morgan, 2010; Ross, 2010). This "otherness" allowed Kristene to experience cognitive dissonance and disorienting dilemmas described earlier. In addition, Kristene's willingness to initiate new experiences throughout the study-abroad program created a condition where she could experience transformative learning as described above (Mezirow, 2000). For Kristene, the studyabroad experience also provided opportunities for cultural and linguistic "otherness" discussed in the literature review (Morgan, 2010; Ross, 2010) and will be described below.

Cultural "otherness." Kristene's plunge into cultural "otherness" began on the flights to Europe. In her journal she wrote that navigating airports was a "pain... because [she] was only allowed to go through certain gates whereas EU people could go through any." Additionally, Kristene attempted to start a conversation with a woman sitting next to her on the trip over to Spain and the woman was startled and looked away. Based on this travel experience, she expressed the concern that she was nervous about going to Rome after the study-abroad program ended, where there was a whole new language that 
she did not speak and a new culture. Kristene also commented in her journal at the unsettling feelings as her host sister and brother stared at her as she unpacked.

Along with other participants, Kristene expressed the concern that she would "look like a tourist." She stated that it was important to her that she be considered a student, not a tourist. I observed her correct someone once when they asked if the group were tourists. In the entrance interview, as well as other informal interviews, Kristene was worried that she "sounded American" as she described culture shocks. To clarify, she did not express this in the linguistic sense; rather, she feared sounding ethnocentric as she discussed certain topics like sanitation, hot showers, the size of their rooms, and how the Spanish preserved food for future meals. Furthermore, in her journal she stated, "I hate the amount of stares that we receive. They can very easily tell we are tourists and not from here, so that makes it more difficult to try and use Spanish when everyone is so willing to use English under the assumption that we don't speak Spanish." Repeatedly, Kristene (and other female participants) expressed that Spanish women dress "classy" to go outside of their apartments. She felt that Dr. Green did not prepare them adequately for this cultural difference. Because it was mentioned repeatedly by Kristene and other participants, I asked Dr. Green what he did to prepare the students for the cultural differences between Spain and the United States. One of the things he emphasized was the difference in cultural norms for dress outside of the house. Regardless of the institutional preparation, it was a shock that several of the participants experienced and created a sense of "feeling like a tourist" or not dressing "classy" enough. She even wrote about the clothing difference after she was done with the study-abroad experience 
and was literally a tourist while travelling through Italy.

Kristene's host family was a source of deep, personal inclusion and "othering" simultaneously. I didn't realize how much she fit in/played with her host brother and sister until I read her journal, however. I feel that her comfort with her host family, due to the similarity of her family or origin and host family helped Kristene develop empathy for, and a sense of inclusion in, the Spanish culture. In her journal she discussed playing games with them, going to the store for bus passes with them, helping them with homework, going to restaurants, and going to different beaches with them. Furthermore, Kristene mentioned in her first interview, that although younger, her host siblings reminded her of the relationship between her brother and her (similar age differences, gender make-up). During our entrance interview, Kristene expressed how surprisingly similar her host family was to her home family when I asked her to talk about how her host family and her family in the United States were different. She said:

They're actually really not. It's almost funny, because my host family has an older daughter.... She's 10. And a younger brother ... who's 8. And, though the age difference isn't quite the same as me and my brother, like, [the] older girl is more studious, quiet, less of a troublemaker, and then you have the younger brother who's really energetic, mischievous, like, likes to get in trouble. *chuckles* And, so it's really ironic that that's basically how me and my brother were. I didn't realize this until like two days ago, but my host-mom is 42 , which means she had Lucia when she was 32 , which is the same age my mom was when she had me... Both my host-mom and my real mom, they're both at the point 
where their hair is graying and they're dying their hair... Both the dads work very, very hard. It's very similar to my family at home. I mean, the lifestyle is obviously different, but the family structure is very, very similar.

According to her journal, Kristene spent almost one-third of her two days in Madrid looking for souvenirs for her host siblings, spent a lot of her free time helping her hostsiblings with their homework, and went on mini-excursions (shopping, going out to eat, or going to the beach) with her host family. She seems to have developed a close relationship with them. Out of all of the participants, Kristene's host family seemed to most closely match her family of origin more than the other participants experienced.

Kristene's closeness with her host siblings also had negative impacts, creating a sense of cultural "otherness." Several incidences provide examples of how Kristene experienced marginalization due to sizism from her host brother. One day, she went with her host family to a fashion store. She did not really like any of the fashions. Her host brother said she did not like it because she was "fat" (by North American beauty standards, Kristine was a very typical size, which may have been why this experience came as such an intense shock to her). She stated in her journal, "It was really embarrassing after it became a continuous joke for him. I know I shouldn't let it bother me, but sometimes it just does. Thankfully, Amy, Jennifer, and Anne are great friends and didn't let me stay depressed for long." Kristene also mentioned in the entrance interview that the Spanish seemed obsessed about weight, "They're very skinny, the entire culture." Her host brother's obsession with weight may have been a replication of his mother's: 
My host-mom is terrified that her son is going to get fat, because he's eight years old and he plays a lot, runs around, and he has a high metabolism, he's not... he's skinny, but he eats a lot, and she's really worried for him, even though he eats nothing but, you know, healthy Mediterranean food... I think it's an honest-toGod fear here.

Likewise, Kristene told me about a friend her host mom had:

My host-mom, has a friend that I was introduced to, and she almost appears like an American mom does, you know? A little bigger around the waist and things like that, but she keeps referring to her as "la Gorda," and she doesn't mean anything mean by it, but it's just an identifying factor because there's not many people like that here.

Although these two examples are from the entrance interview, I observed her make repeated comments about her perception that the Spanish obsession with thinness throughout the program. Additionally, her journal shows that it was something that caused her great pain and caused her to need to seek out the help of friends. JohnsonBailey and Alfred (2006; see also Johnson-Bailey, 2012) posit that marginalization may be a powerful transformer. Thereotically, this encounter with cultural and social "otherness" through sizism might have been an opportunity for transformative learning and served as a window to see other marginalized groups through a lens of empathy. However, it is impossible to deduce from the data collected what the long-term effects will be on Kristene or on her awareness of and empathy toward other marginalized groups. 
Linguistic "otherness." Although I observed that Kristene had the best Spanish of the group, she experienced linguistic "othering." One of Kristene's difficulties during her study-abroad experience was that she would talk to people in Spanish, they would reply back to her in English, which was a huge disappointment to her. In her exit interview, she stated:

I remember at one point Dr. Green did say, "You could probably go to Spain and get away with not using Spanish at all.” But... that was kind of a disappointment to realize, but, on the other hand, I've also been trying to use my Spanish rather than English. So, most people understand that, "Okay, well I'm speaking Spanish to you, so I want you to Spanish back to me." Not turn it around and speak English. So, that was the only real shock is that they sometimes will just assume you don't speak Spanish at all.

Kristene described another linguistic epiphany that happened to her while she was helping her host sister with English in her journal. She realized that the host sister would probably never get English pronunciation perfectly, just like she would never get Spanish pronunciation perfectly.

These experiences do not seem dramatic. However, in her member check interview several months after the study-abroad program ended, Kristene noted that the linguistic "otherness" she had experienced encouraged her to seek either a certificate or a master's degree in teaching English to speakers of other languages (ESOL).

Kristene's socio-cultural outcome of desiring to become a more linguistically responsive and culturally relevant teacher echo Foster's (1997) assertions that learning a 
second language can create a "profoundly unsettling psychological proposition" wherein the participant's "immediate interaction with the language and culture can directly threaten an individual's self-concept and worldview" (p. 35). Likewise, Goulah (2007) found that found that students who learned another language while immersed in that culture developed critical socio-cultural attitudes about their home culture. Kristene's increased capacity to situate herself within the larger political, economic, and sociocultural domain by seeking an ESOL certification is an important outcome of this study (see Coghlan \& Gooch, 2011). From the experience of taking the linguistic and cultural risks in Spain, Italy, and France, Kristene's desire to develop skills to more competently respond to linguistically marginalized students is a sociocultural outcome that aligns with scholars who maintain that individual perspective transformation is not complete without the transformation benefitting society (see Brookfield, 2012; Cranton \& Taylor, 2012; Johnson-Bailey, 2012; O’ Sullivan 2012; Taylor, 2012).

It is impossible to prove, but likely, that the marginalization that she encountered with sizism, linguistic "othering," as well as her growing political awareness of marginalized groups contributed to her desire to teach students who are learning English in the United States. Furthermore, from Mezirow's (2012) description of incremental transformations as "a progressive series of transformations in related points of view that culminate in a transformation" (p. 86) and Tisdell's (2012) findings that incremental changes, over time, can lead to equally as poignant of a transformation, I maintain that many of the experiences with cognitive dissonance, linguistic and cultural "othering," as well as Kristene's newfound sense of independence worked together to generate an 
opportunity for Kristene to transfer her personal transformations to the benefit of others in society.

What is each student's racial, economic, gender, and cultural identity? How does each student's identity impact her worldview and transformations (or lack thereof) during the study-abroad program? As mentioned earlier, Kristene was twenty years old at the time of travel. She informed me in the entrance interview that her parents paid for the study-abroad experience and the two-week journey around Europe after the study-abroad program. Kristene indicated throughout the study-abroad program that although she was politically liberal, she recognized that she grew up in a comfortable, upper-middle class environment. She understood that it was a privilege to have parents who could afford to send her to study and travel through Europe for six weeks. She self-identified as a White, heterosexual, cisgender female. Additionally, some of her conversations revealed that she at least grew up around the privileged class. For example, she revealed that she had multiple cousins and friends who had studied in Europe (including one friend who came to visit Levante during the festivals who was studying about 40 miles away).

I found Kristene to be rather self-reflective, even when it ran counter to her own biases. For example, even with the disdain she had for the bullfight mentioned above, after describing how horrific she found it, she stated, "But I eat meat." She often recognized the recognized when her views and her actions did not coincide.

One fascinating thing was that Kristene discussed how much things cost and money more than other participants. At the beginning of the study-abroad program it 
made me wonder if it was a financial hardship for her to attend. However, later, I found out that her parents had paid for the trip. One example of her focus on money occurred when one of the participants asked me where I lived in Levante. I told her that I lived with Dr. Green because his family did not come. Kristene started complaining saying, "I hope I did not pay for you to come here. I hope my fees do not pay for Dr. Moss's apartment. I definitely hope I did not pay his airfare...I had to pay for my own airfare."

As I analyzed the field notes, I saw a recurring theme that she often talked about money. It seemed that since her parents paid for the trip and that they had enough for her to go to Italy, France, and England afterward that it would not be a big deal. Kristene and Jennifer were the ones who discussed money most, yet neither of them came from families of economic struggle.

Another fascinating personal preference that might have class implications was her desire to go to Spain to learn Spanish rather than somewhere in the Americas or Africa. When I asked in the entrance interview why she decided to come to Spain, Kristene responded, "it was always Spain, it was never South America or Central America, it was always Spain. I really don't know why, just always was.”

Analysis of independence epochal transformation through the socio-cultural variables of race and class privilege. Kristene experienced a sizable paradigmatic shift in developing a personal sense of independence, which could be linked to the humanist axiology of TLT (Mezirow, 2012). As individuals become increasingly autonomous and reflective, they move toward consciously constructing personal knowledge/assumptions and become increasingly responsible for their actions (Taylor \& Elias, 2012). From a 
humanist axiology, Kristene's developing sense of independence aligns with TLT's paradigmatic underpinning that humans have a natural desire for freedom and autonomy (Cranton \& Taylor, 2012).

However, her newfound independence and autonomy could also indicate race and class privilege. As explained in the literature review, Johnson-Bailey and Alfred (2006; see also Johnson-Bailey, 2012) posit that marginalization may be one of the most powerful transformational experiences. Moreover, E. W. Taylor (2007) argues that socio-cultural context of the participant (race, class, gender, sexual orientation, and culture) may be the most important variable on transformative learning outcomes. As I analyzed the data, I observed that the higher the class privilege of each participant, the more likely they were to be dependent upon, or complain about Dr. Green, their host families, and the LU staff. The lower the students fell on the socio-economic spectrum, the more likely they were to figure out things for themselves and be content with the provisions, activities, and living arrangements of the LU study-abroad program.

Likewise, the two students who self-identified as Latinas (one lower-middle class, one who grew up in poverty) were more independent and content throughout the study-abroad program, showing a connection to racial privilege as well. However, because the sample size of this study is small $(N=8)$, and the number of Latina participants was even smaller $(n=2)$, it is impossible to tease out whether this trend was correlated to class or race privilege, or both. Nevertheless, while marginalized students (in this case previously marginalized due to class and/or race) are confronted daily with inconsistencies between social ideals and lived experiences (hooks, 1994; Johnson-Bailey \& Alfred, 2006), 
persons in positions of privilege (in this case upper-middle class, White Kristene) have difficult cognitive and emotional work to break free of prejudiced ways of seeing the world (McIntosh, 1990; O’Sullivan, 1999). Consequently, because society privileges White, upper-middle class individuals (Leonardo, 2009), social structures are designed to allow transformations of this type to be merely personal, while individuals who lack privilege must develop this autonomy to navigate an unjust world (Johnson-Bailey \& Alfred, 2006). Therefore, it is possible that Kristene's newfound sense of autonomy might have merely reflected a previous lack of racial or class marginalization or financial necessity to be independent.

An example of Kristene's reliance upon authority happened during one of the last days of classes. Dr. Green and Juan informed them that the bus schedules change during the festival that happened the last weekend of the program. Kristene asked Dr. Green to tell her what the new schedule/stops would be. Dr. Green informed her that it was impossible for him to memorize all of the stops and routes for a city of 300,000 inhabitants. He encouraged her to Google the city's website, because the new routes and schedules were posted prominently on the website. The following day, Kristene arrived to class about 30 minutes late and expressed frustration to her classmate that nobody told her the new routes/schedules. Similar events happened for Anne and Jennifer, who selfidentified as upper-middle class. It is very possible that this study-abroad program represented one of the first times that Kristene had to figure out things on her own.

From a critical social stance, this sense of independence may also be a sociocultural awakening (or re-awakening/deepening of previous values). In her exit 
interview, Kristene stated that the political discussions in both classes and her observations of gender and LGBTQ+ freedoms in Spain made her "even more liberal than [she] already was." Curry-Stevens (2007) concluded that part of TLT being a "pedagogy of the privileged" is making space for individuals to reconnect to greater social causes, rather than to tie social ideologies to individuals of similar socio-cultural variables. Likewise, CRT scholars contend that socio-cultural awareness of marginalization leads individuals to validation and affirmation, which, in turn, hopefully leads to social action (Delgado-Gaitan, 1993; Ladson-Billings \& Donner, 2005; Smiley \& West, 2012).

It is evident from this study that Kristene experienced a deep, personal transformation toward personal independence. For her, the study-abroad program was life changing. This change matches Mezirow's (2000) description of transformative learning. However, the most repeated critique of TLT is that it is overly focused on individual transformation and does not focus directly on social transformation (Tisdell, 2012). Critical social TLT, however, goes beyond merely empowering participants to change their lives for the better and insists that participants act to improve society, as well (Merriam \& Kim, 2012). Stated differently, a critical social TLT perspective requires individual epistemology to be tied to political action (Brookfield, 2012). However, I cannot deduce from the data collected during this study what her intentions were or how this sense of freedom will impact her social conscience, or more importantly, her social action, over time. 
Participant Two-Jennifer—From "Deer in the Headlights" to "Bubble" Breaker

"Jennifer" was twenty years old during the study-abroad program. She was in between her sophomore and junior years of college, with a major in political science/prelaw and a minor in Spanish. Previous to the study-abroad program, Jennifer had travelled throughout the Midwest, Great Plains, and Rocky Mountain regions of the United States. Additionally, her parents had taken her to several places in the Caribbean. However, during the trips to the Caribbean Jennifer stayed on houseboats and only did minimally touristic adventures, so Levante was her first experience being immersed in another culture. One important demographic difference from other participants is that Jennifer was from a city of 300,000 people that was substantially geographically removed from the metropolitan area where most of the other students were raised. While the other major city had a metropolitan area of several million inhabitants, Jennifer explained in the member-check interview that most of the participants from that area were from White, upper-middle class suburbs that were "cities unto themselves" in Jennifer's words. She maintained (and Wikipedia/US Census Data confirmed) that the suburbs that other students were from were about 100,000 people and were over 90\% White. While Jennifer did not argue with being considered upper-middle class, she felt that her city and schooling experience gave her exposure to racial, class, and sexual/gender diversity that other participants did not experience.

Jennifer was always extremely well prepared for presentations in Juan's class. Juan typically sent students a basic outline in PowerPoint format for class presentations. I observed in my field notes that Jennifer added content (pictures, video clips, 
streamlined the animations) to Juan's presentations. I wrote that it was "obvious she had done extra background investigation" over Juan's outline. While she tended to read the content, the extra material made her presentations stand out from other students. Moreover, she dressed in a "business dress" style, which Juan commented to the other students was "typical for doing presentations" in Spanish universities and congratulated her on observing cultural presentation norms.

Jennifer always sat in the front row of class. I wrote in my field notes that "Kristene and Jennifer always give 'good student' answers." When Jennifer was excited about a topic, she would sit up straight, eyebrows raised, and her whole body would become energized. I wrote, "Jennifer really gets the class going when she gets excited about a topic." Similar to Kristene, Jennifer would get excited about a topic and then dominate a discussion for a few minutes. In contrast to Kristene, it was not Jennifer's linguistic ability that allowed her to be the main speaker for a couple of minutes. Rather, it was her intensely excited body language, enthusiasm for the topic, and raised voice. Furthermore, Jennifer's excitement often led to added discussion and engagement by other students, while Kristene's Spanish ability and personality tended to shut down discussion. Consequently, both professors utilized Jennifer's enthusiasm to engage other students in class discussions. Jennifer's comments often ended with a "What do you all think?" Whereas Kristene's comments had an authoritative, "I'm right" feel to them. What surprised me, observing Jennifer's academic skills, was how Jennifer complained anytime they had to do something that required the use of a computer. I found that to be contrary to the meticulous student I observed over almost five weeks. In 
the first week of classes, I wrote in my field notes after hearing Jennifer's complaints about having to do a PowerPoint, "as [a meticulous] student, it makes me wonder how they came here without laptops or tablets to do homework." In preparation for the member-check interview, I wrote an email to Dr. Green to see if he may have an insight as to why so few of the students brought laptops to a study-abroad program. He wrote back and informed me that he instructed his students not to bring technology. He maintained he did this for two reasons: 1) He wanted them to use the LU's computer lab to get them to interact with other Spanish students, and 2) while violent crime is extremely rare in Spain, petty theft is commonplace. Students on previous trips had computers or tablets stolen, so Dr. Green encouraged them to utilize the resources at LU. Jennifer confirmed this in her member-check interview.

As a researcher, I came to enjoy Jennifer's outgoing personality. She was very frank about her opinions, both positive and negative. In the member-check interview I asked her about her open, candid personality and she explained:

I have just really become a person who says what's on my mind and tell you like it is ... I would rather you tell me upfront and hurt my feelings then not tell me or lie to me and say things behind my back or whatever. Just say it like it is, I will tell you like it is. I'm a very blunt person. I think that's the best approach. And actually it worked out because that's how Spaniards are, too. They are very upfront. They don't see a reason to hide how they feel or tell you something different or anything like that so I am very open. 
Because of Jennifer's transparent and outgoing nature, I collected over 50 pages of field notes, interviews, and observations on Jennifer.

Elements of TLT Jennifer exhibited during study-abroad program. I will begin this section explaining findings relating to cognitive dissonance (culture shocks, culture adjustments, and culture surprises) Jennifer underwent during the study-abroad program. Subsequently, I will describe the incremental and epochal transformations Jennifer experienced.

Cognitive dissonance. Prior to the study-abroad experience, Jennifer and Kristene were close friends. Additionally, she had taken several classes with and was on a first name basis with Becky and Anne (discussed later). Like her good friend Kristene, Jennifer found navigating airports to be one of the biggest culture shocks. In the entrance interview, Jennifer explained, "The airports were a lot harder to navigate than we thought. We never imagined they were going to be that hard." Two weeks later in the focus group interview, she reiterated this culture shock:

The Madrid airport was an absolute maze. We had nobody to help us, no headsup. People knew we were flying through Madrid, and no one decided to say, "You should maybe make sure you're very time-efficient. It's gonna be difficult.” We also, when we got to Madrid, we had to go through customs. Nobody told us that, either.

Jennifer mentioned several other times that this experience was "unsettling," and that she was "going through an airport completely blind," and that it was her "first time our of the country," and she had "no idea what [she] was in for." I believe this may have been one 
of the first times in her life that she experienced cultural and linguistic "otherness," which will be discussed in greater detail in the next major section of these findings.

Spanish all the time. I noticed during the student orientation meeting and during the first week of classes that Jennifer was focusing so intently she had a "deer in the headlights" look. Because her body language often gave away her emotions, I also observed that she consistently increased comprehension skills as the study-abroad program progressed. I mentioned in between classes during the first week that I could see that she was focusing extremely hard and empathized with her because I remember that feeling the first couple of months I lived in Spain. In her member-check interview she said that listening and speaking in Spanish all of the time "was definitely something to get used to."

"Public displays of affection" and nudity. When asked what surprises she had experienced her first week in Spain during the entrance interview, Jennifer stated, "PDA [public displays of affection] here is really something that's just passed by. No one takes a second glace. But in America, if I saw someone doing that on the street ... I would be very offended." Furthermore, in her member-check interview Jennifer maintained that watching teens and young adults making out in public spaces was shocking and unpleasant to her during the first couple of weeks she lived in Spain. She said:

The first day that we were in Spain we went to the beach ... And not only did I see topless women...but there was some other ... things on the beach things that women were wearing that I had never seen the likes of in public... Kristene and I will joke about [it] forever because it was just interesting and the same thing with 
PDA. 14-year-olds on the side of the street just you know going at it ...that was something that I was not prepared for because [in the United States] that is not okay.

Differences in lifestyle between Spain and the United States. Jennifer mentioned several times that "America was more materialistic" and that Spanish life was more "simple" and "laid back." In the entrance interview she stated she thought: everywhere in the world is just like America, when, in fact ... nowhere really is.... Because America probably focuses on the glamorous, ... material things way more than they should.... That was surprising in a good way, because it helps me realize what I take for granted.... And so I think when I go home it'll be, eyeopening to what I appreciate more.

Jennifer reiterated this idea in different forms in both her exit interview and her membercheck interviews. One example that illustrates this idea occurred in Juan's class when he asked the students what surprised them most about living in Spain. Jennifer expressed this idea and stated that one of her biggest surprises was finding out that almost everyone lived in apartments. She told him she had pictured everyone living in little white houses with Spanish tile roofs.

Additionally, the way women dress and the way she felt like she was expected to dress was a surprise to Jennifer. She explained to me in her entrance interview:

Everyone has to try to look nice every day. And that's very different, especially in college. There's days I would roll out of bed, throw on this outfit, put my hair in a ponytail, and just go. And I can't do that here. That is not acceptable.... I 
didn't expect half the women I see on the street to be wearing heels when they have to walk a mile to work.

Jennifer also commented several times in informal interviews that she felt that the women in Spain dressed "classy."

Self-diagnosed "germaphobe." In the entrance interview, Jennifer said that one of the hardest things for her to get used to in Spain was how food was prepared, handled, and stored. For example, in the entrance interview she said, the host family "put the leftovers just on a plate [uncovered] in the fridge and just let them sit, especially because the doors are opened at all times ... so bugs have complete freedom to all of our food. And, then I remembered that I'm probably going to be eating that in another day or two." Additionally, most Spanish meals are accompanied by a baguette of bread. About the custom, Jennifer stated:

Usually when a food touches the table, I'm like, “Oh, can't eat that anymore.” But this bread just sits on the table, all the time, and everyone grabs and tears.... Everyone's hands have been all over this bread....'Cause you do not do that in the U.S., that is not okay.

In the member-check interview, Jennifer said, looking back, "The bread I got over. But the food storage I will never get over... the idea of it really grossed me out, and I can do nothing about it because it was my dinner for the day so I just had to eat it anyways." Besitos. Jennifer shared Kristene's initial hesitation to participate in the besitos tradition of greeting people by touching cheeks and making a kiss sound. Jennifer was the participant that brought up the fact that Dr. Green and I gave the Alhambra tour guide 
besitos by saying, "You just don't do that." However, when Manolo gave her besitos at the end of the Granada/Alhambra cultural excursion, she participated in the cultural tradition. Over time, I observed that Jennifer was more concerned about the cultural appropriateness (when to give besitos and when not to do it) than she was about the practice, in general. When I asked her about her initial shock in the member check interview, Jennifer explained:

“Obviously at first it was strange because we don't do that [in the United States.] ... Once you grasp the fact that it is so normal and not even a big deal at all then ... if they initiated it, it was not a problem. But I wasn't going to be the one to initiate it."

Water conservation. On her first shower in Spain, Jennifer told me she was shocked when the water went cold after seven minutes. She stated in the entrance interview, "That's not adequate time to wash all my hair, wash my body, shave my legs.... And so you have to do this start-and-stop system. Very different, again. A little hard to get used to." During the exit interview she said about the practice, "Maybe when I go back, I will take quicker showers." In the member-check interview she said that her hopes to conserve water "died very quickly" upon returning to the United States.

Difficulty of classes. Jennifer consistently mentioned between Juan's and Ana's classes that the exams, homework, and presentations were "crazy" and "too much." She additionally expressed the concern to Dr. Green in one of the focus group meetings. Other students (ones who struggled much more in Spanish that Jennifer did) told Dr. Green it was not as bad as she portrayed it to be. In Jennifer's defense, I observed that 
other students did not give the same quality of presentations that Jennifer did. Similarly, Kristene and Jennifer expressed concern in the last focus group meeting that the professors had not collected anything up to that point. Dr. Green encouraged the students to take the final exams very seriously, because they typically were a large part of the grade in Spanish university classes (Dr. Green received his master's degree from Levante University). During the final exam, when Ana wasn't looking, Jennifer did a gun to the head sign with her hand and "pulled the trigger." She also complained to the professors that they had to do presentations their last couple of days in Spain. I could never decipher from the interviews whether the classes were harder than the PSU classes Jennifer was used to or whether she simply expected the classes to be much easier than they were.

Bullfight. Jennifer was one of the more vocal opponents of the idea of having to go to a bullfight as part of her grade. Moreover, she was one of the students who left after Dr. Green allowed the students who were uncomfortable to leave during intermission. When I asked her what she thought of the bullfight in her exit interview, she said:

It was even more horrific than I anticipated. I honestly anticipated to not be able to see the gory parts, and that's all I could focus on. I hate it even more now than I did before... It's forever going to be a part of the culture I just can't accept. When I asked Jennifer if the United States had something as horrific as bullfighting in the exit interview, she thought the idea of dogfighting was comparable, but quickly noted that dog fighting is illegal in the United States whereas bullfighting was 
celebrated (at least in the arena where we watched the bullfight) in Spain. She went on to explain that she had a tense debate with her host dad about the practice of bullfighting afterward. She explained that her host dad said, "When they get in the ring, it's either the bull or the man." Then, they then discussed politics and she found out that her host dad was extremely conservative, for the monarchy, and probably was a Franquista (a person who thinks society was better during the fascist dictatorship of Ferdinand Franco). From her comments, it was evident that she associated his social conservatism with the violence and gore she watched in the bullfight.

Cultural adjustments. Because Jennifer was so transparent, she mentioned many minor culture shocks that would not probably qualify as cognitive dissonance. I include them here as cultural adjustments. For example, during one of Juan's classes Jennifer said that she was shocked that fast food restaurants were allowed to sell alcohol. Additionally, she explained that it took her a few days to get used to not flushing the toilet every time she went to the bathroom (even describing her fascination with a half and full flush button on the toilet). Lastly, during her first week in Spain, Jennifer said it took some time to figure out which restaurants had debit card readers and which ones required cash payment.

Cultural surprise. Like her friend, Kristene, Jennifer felt the words "culture shock" had a negative connotation, so she preferred the term "cultural surprise" for good things she observed in Spanish culture. One of these was the festival that lasted for five days during the final weekend of the program. In the exit interview, Jennifer told me that "as far as the fiestas go... the Fourth of July will never be the same.... That was the most 
beautiful firework show I have ever seen in my whole life, and I will never see one like it again." She seemed to have a positive transformative experience during the festivals.

One of the reasons that this weekend was so special to Jennifer was the sense of inclusion that she felt from her host "sister," "Magdalena." This relationship will be described in the section below on cultural inclusion.

Another cultural surprise that Jennifer expressed several times was a perceived difference in lifestyle between Spaniards and North Americans. One time Ana asked why the students thought the life expectancy was higher in Spain than it was in the United States. Jennifer answered that the diet of Spain was considerably different than it was in the United States, especially in that there was much less fast food available. She said that she had only seen three fast food chains in Spain, whereas in on street by her home in the United States there are 10 different fast food restaurants.

\section{Incremental transformation: Deepening of socio-political consciousness. As}

mentioned earlier, Jennifer's whole being would energize every time something political was discussed in class (feminism, sexuality, capitalism, and forms of government). I first noticed this phenomenon at the beginning of the second week during a discussion in Ana's class. Ana was lecturing about the impacts of the Spanish Civil War on an artist and was trying to get the students to think beyond the political binaries of Democrat/Republican (in the United States). Ana asked Jennifer to explain what communism was and Jennifer explained in the United States communism is considered "bad," which reproduced the metanarrative about socialism or communism put forth by many textbooks in the United States. After a brief discussion (which will be discussed 
further in Tim's Findings section), Eva explained that communism might be a more "radical" system, but that in Spain it is not socially acceptable to call any political system "bad." Rather, they should discuss the ideas with which they identified or disagreed. Ana then asked the students if capitalism works. They all (except for Tim) nodded with their heads in agreement. Ana countered, "Maybe you should ask a child in Ghana or Algeria how capitalism is working for them." Furthermore, maintained that capitalism gives resources to one person at the expense of others. I could see from Jennifer's reaction that she had never been encouraged to think of the negative influences of capitalism before.

Juan's class often tied politics into the history and culture of Spain as well. In the third week, Juan was talking about feminism during the time of Franco. He explained during the fascist dictator's rule, a woman could not own property, could not open a bank account, and could not do anything institutional without the express authorization of her father or husband. Jennifer countered that it was like that in the United States at one time. I wrote in my field notes "it is exciting that she is connecting the United States politics in Spain." Juan responded that this was from the 1930s-1970s. One thing that got Jennifer particularly frustrated was understanding the $d e$ (of/from) in Spanish last names. It indicated that the husband owned the wife. Jennifer commented that she found this idea especially repugnant. I wrote in my field notes the question, "I wonder if Jennifer will take her husband's surname when she gets married."

Slowly, Jennifer's discussion of politics changed from a binary "good vs. bad" or "right vs. wrong" to a more socio-cultural worldview. One thing I am not able to fully analyze from the data collected is to what degree her improved Spanish skills played in 
the nuance of political conversation. However, it was not her choice of words that changed. Rather, her political discussion gained nuance and complexity. Therefore, I believe that Jennifer's political views became more culturally and socially multifaceted. I wrote in my field notes on the last day of classes about Jennifer and her presentation: I really believe that [Jennifer has] gained more in a more permeable/open-minded worldview than [she has] in Spanish. [Jennifer] understands much better than when we came here, but her [spoken Spanish] is pretty similar [to when we arrived]. However, I was intrigued at [her] capacity to debate politics, religiosity, and other complicated subjects during the [final] presentations of Juan's class. She went beyond the Left vs. Right stuff [binaries common] in the United States. She showed concerns/deep thinking in the way she talked about Catalunya and País Vasco wanting to separate [from Spain]. She also discussed the political correctness of the religiosity in the United States versus the festivals in Spain. It was not the depth of the argument that impressed me, but [her] willingness to consider new ideas.

One example of Jennifer's growing socio-political complexity happened during the last week of class. Part of the Levante festivals includes burning huge papier-mâché statues that cost upwards of $\$ 150,000$. Juan asked if it was "immoral" to burn something that costs between $\$ 50,000$ and $\$ 150,000$ dollars each (there were dozens of them). Jennifer explained that she thought it was a partly immoral since there was a financial crisis with about $25 \%$ unemployment while we were in Spain, but she also admired the fact that it was a tradition that people had celebrated for millennia no matter 
what the financial situation was. Juan stated that most people considered it an investment because it brought much more money in tourism than it costs. It was in this discussion that I started to notice her ability to articulate a more nuanced political stance.

In another example, when other students were criticizing how much the monarchy in Spain cost during class the final week, Jennifer said that she did not like that presidents and congress people of the United States receive a salary for the rest of their lives. She countered, 'Is that not a form of being a king?" Juan, excited that Jennifer was bringing in a critique of the United States (subjective reframing rather than merely objective reframing) said, "Should they be allowed to work for private companies during and after their terms?" This led into a powerful discussion about whether money should be considered free speech.

During her member-check interview, Jennifer talked to me about this incremental change toward nuance, critical reflection, and subjective reframing:

I have very liberal views.... I guess being in Spain where [many] also have ... very liberal views... confirmed my feelings about how much I think that the [United States] needs to change.... If anything it only lit more of a fire under me and my beliefs. It probably just enhanced what I feel and what I think.

Jennifer went on to explain that she had taken the most major intense semester of her political science major and how much the study-abroad program helped her cement her liberal political frame of reference. She maintained that the study-abroad program gave her a "worldly view" rather than a "US view." Furthermore, Jennifer maintained that the study-broad program instilled in her a desire to "delve into and to learn more about 
other... political systems." Additionally, she said she became very interested in how different political systems compared in different countries and wanted to visit other countries to experience how their system of government influenced the style of life in those countries.

Jennifer also expressed a concern of the amount of time that was spent about the United States in Spanish news broadcasts. In her exit interview she stated:

The United States is constantly on the news. And I said that, I mean, that comment once, to my host-mom, and she flat out said, "It's because the United States is the most important country in the world." And that just took me, it took me aback. One, to hear a Spaniard say it. Two, someone just of a different country say it. And three, because I had fully-convinced myself that we were not; in fact, we kind of - I mean, yes we help a lot of people, but we also hurt a lot of people. Jennifer stated that she had never fully realized how much importance the United States government and policy impacted and concerned other nations. Although she did not use the term "colonialism," I could sense in this and other interviews (as well as in comments in Juan's class), that Jennifer was becoming aware of, and concerned about the role of cultural colonialism the United States has imposed on other countries.

Jennifer's growing political awareness showed a growing capacity toward subjective reframing and critical reflection. As mentioned in the literature review critical reflection requires students to understand the intentions, purposes, feelings, values, and moral decisions behind ideas (Mezirow, 2000). Subjective reframing involves critical reflection about personal assumptions and critical examination of the reasons for limited 
personal frames of reference (Mezirow, 1998). In Jennifer's case this subjective reframing was more about the United States' political system, which matches Mezirow's (2012) claim that subjective reframing can be about systems, organizations, or institutions in addition to personal frames of reference. Jennifer's transformative experience about colonialism was most likely spurred by in-depth discussions that she had with her host family every night as they watched the news together and discussed politics (Ross, 2010). Moreover, Jennifer explained in the exit interview that her host mother was politically liberal and her host father was very conservative, which created many opportunities for them to discuss politics.

Epochal transformation: "Bubble breaker." From the entrance interview through the member check interview, Jennifer discussed her need to "break out of the bubble" of being at home and at PSU. In the entrance interview she told me that she felt that attending a study-abroad program was a "now-or-never opportunity." Her desire for something novel and life-changing aligns with Morgan's (2010) findings that willingness to be changed by the travel experience increases when there is a specific purpose behind the travel. It also parallels Ross's (2010) findings that novelty of the destination increases opportunities for transformational learning.

Jennifer maintained that the study-abroad program made her think about her future, "This whole trip has really made me open my eyes to a lot of things." During the exit interview, Jennifer felt like this experience had been a "turning point ... to make more of my life." She went on to explain that she hoped to learn more languages, travel to more countries, and experience other cultures. Furthermore, Jennifer stated the study- 
abroad program cemented her desire to attend law school and added a desire to transition more quickly to law school than she previously had planned:

My views are very different of what I want to do after [PSU].... I want to move on more quickly then I maybe I previously had.... I want to go to do things and go to bigger cities in different places.... After being in Spain ... I don't really have that many concerns ... with picking up and moving to another city.... [The study-abroad program] was the greatest thing that I've ever done."

Another "bubble" that Jennifer broke out of, was a willingness to try new things. Her desire to grow and experience new things was visible from many of her actions. For example, on the first official day of classes, there was a welcome reception, where typical foods of Levante were offered to the students. I wrote in my field journal, "Jennifer consistently surprised me with her willingness to learn and to try new things.... For example, at the opening food reception, she said hated fish/shellfish, yet she tried everything." In the member-check interview, Jennifer said that living in Spain caused her to want to be open to trying even more experiences: "Before this trip I was not an adventurous person. If you put weird food in front of me I would not try it. But I guess at some point I decided I would make the most of the experience because I did not know when I will be back."

Both Jennifer's growing political awareness and desire to "break out of the bubble" align with K. Taylor and Elias' (2012) explanation of subjective reframing as a person being subject to that which is "larger" than her. To be larger than a person, the thing or idea must be more complex or bigger than that individual's ability to understand 
or change the item. Likewise, things that are "smaller" than a person become object to that person. To be smaller, the item or idea must be less complicated, meaning the individual can look at it, take responsibility for it, reflect upon it, exercise control or manipulate it, and change it to a new way of knowing. By travelling to Spain, the world outside of the medium-size, rural PSU became "object to," or "smaller" than Jennifer. She understood and was no longer intimidated by other cultures and places.

Epochal transformation: "Day by day." During the final interview, Jennifer maintained that living in Spain had taught her to live "day by day." She felt that she had taken everything too seriously in her life before the study-abroad experience. In the exit interview, Jennifer stated that "the Spanish culture ... taught me ... every day's a new day, don't take life for granted." Jennifer stated that this was "a completely different mindset" than she had prior to the study-abroad program. Later, I will discuss Jennifer's intensity to take minor personal setbacks as a failure of the study-abroad program. Before the study-abroad program, she stated that she "was never really good at separating" leisure from work time. Now, she felt "there's a time and a place for everything ... and the Spanish people truly live by that.... When it's time to party, it's time to party. When it's time to work, let's go to work."

What role did encounters with cultural and linguistic "otherness" play in the transformative learning (or lack thereof) of each participant? In the following section, I will discuss Jennifer's experiences with cultural "otherness," cultural inclusion, and linguistic "otherness."

Cultural "otherness." In formal interviews, classes, and her conversations with 
classmates, Jennifer discussed how difficult it was feeling like an outsider. In her entrance interview she stated:

I think the hardest thing to adjust to is walking around every day knowing everyone knows you're different. And everyone is constantly judging us. It's kind of hard because ... in the [United States], you just ... do your thing and no one really takes a second look. But here, it's like everything I do is constantly being watched. And it's just uncomfortable."

Jennifer also mentioned in that interview that she always had to be aware of what she was wearing, what she was carrying with her, and what language she was speaking to avoid "looking like a tourist." Furthermore, in the exit interview she said she "hated being started at, and getting funny looks everywhere we went." Jennifer also repeatedly worried about "sounding so American" throughout the program.

About three weeks into the program, Jennifer and a couple of the other students asked Dr. Green why they were not meeting more Spaniards. They expressed disappointment that the program did not grant them more opportunities to meet Spanish students their age. Dr. Green encouraged them to get out and meet people in the same ways that they would meet students in their own college town. A few minutes later in the focus group interview Jennifer said,

If I wanted to go out to a bar often and just throw myself into other people's conversations and say, "Hey, be my friend," I could do that, but ... I don't feel like that's really okay here. Especially, I don't speak their native language. So, 
I'm going to go in there, stumble around a conversation, make a fool of myself, and they're just going to laugh at me and think I'm a stupid American.

It was evident from these and other incidences that this was the first time Jennifer had felt "othered" in society. However, she never seemed to make the subjective leap to how marginalized individuals are culturally "othered" in the United States and how her own epistemology might reinforce that marginalization (below, I will discuss how she did experience subjective reframing and subsequent social action regarding linguistic oppression). Jennifer's lack of subjective reframing in this area parallels Brookfield's (1998) statement about finding flaws or contradictions in one's own assumptions as a difficult, puzzling, and near impossible task to do when an individual benefits from racial, gender, or sexual privilege. Similarly, Jennifer's politically liberal stance void of observed or self-identifying personal responsibility aligns with CRT scholar Leonardo's (2009) quote about liberals:

Who are these bad whites? It must be the position of a good white person to declare that racism is always about 'other whites' ... This is a general alibi to create the 'racist' as always other, the self being the exception. Since very few whites exist who actually believe they are racist, then no one is racist and racism disappears more quickly than we can describe it. We live in a condition where racism thrives absent of racists. (p. 267)

Cultural inclusion: “Magdalena.” Although Jennifer's first three weeks were full of a lack of subjective reframing, the festivals that lasted for five days in between the third and fourth weeks of the study-abroad program created a major shift in Jennifer's 
worldview and view of the program in general. Her host "sister," Magdalena, came home from another city where she was studying. Magdalena was approximately the same age as Jennifer. She had come home to experience the huge festival of her hometown, Levante. Magdalena took Jennifer to discotheques, neighborhood block parties, food events, fireworks, and showed Jennifer (and several other of the participants) how Spanish students enjoy the festival. At first, Jennifer thought "it was going to be super awkward ... But, we ate dinner together, just the two of us, and then she wanted to go out, "cause she, obviously, came here to party." Jennifer was impressed that people of all ages partied until 5:00 or 6:00 in the morning. In the exit interview she said, "Old people were just dancing their life away." Jennifer loved going out with Magdalena because her host sister knew "authentic" (rather than touristic) places they could go and celebrate the festival with other Spanish students. After spending five days with Magdalena, Jennifer stated in her exit interview, "I truly have come to feel like a Spaniard ... and I really like it. I like being able to walk around the city, and just feel like it's where I live.... It's my home." Additionally, after this weekend of inclusion, Jennifer stated she "never want[ed] to leave" and that she was not anxious to return to life in the United States during the final interview.

In the exit interview, Jennifer labeled this weekend as "the turning point" in her views of Spanish culture, the study-abroad program, and in her decision to study out of state for law school. Before the study-abroad program she felt like a month was extremely long to be away from home. However, after this weekend she said that she 
wished the program had lasted an entire semester, or maybe even a year to allow her to get to know the culture and people of Spain better.

Linguistic "otherness." All of the students (except Tess) struggled with transitioning to Spanish only during the first week of classes. I described the professors' speech in my field notes as "meticulously enunciated" and "painfully slow." Like mentioned earlier, I also wrote in my field notes that Jennifer looked like a "deer in the headlights" the first few days. I could see that she was trying intently to understand, but was struggling to understand much of anything (which she confirmed in an informal interview between classes the second day of classes). The tour guides spoke slowly as well, but I observed from the students' body language and side discussions that they typically ceased trying to listen after the first few minutes of the cultural excursion tours. In the exit interview I told Jennifer that the professors and the cultural coordinators spoke extremely slowly in the beginning, while most of the PSU students looked confused. She replied, "What's funny about that, is I never noticed it! Obviously." She also said that even though the change to listening all day is Spanish was challenging, she said, "if anything [I] appreciated it, because it definitely helped [me], [in] transitioning into only Spanish.”

One thing that made it apparent that Jennifer was comprehending Spanish better was that she started to participate more by the end of the first week of classes. I observed it was her natural disposition is to participate in classes, so it was evident that she understood more by her increased participation (which she confirmed in the exit interview). By the middle of the second week, I observed in my field notes that Juan and 
Ana spoke at a "natural pace." Most of the students appeared to be understanding better and interacting more with the professors by this time.

When I asked Jennifer in the member-check interview how she felt her Spanish had improved on the trip, she stated that during the study-abroad program her Spanish grew "leaps and bounds." She felt being "immersed" in the language and being "forced" to speak Spanish "was exactly what I needed. Because ... I think I've taken Spanish for too many years now. I think I've hit a plateau. Just being there ... skyrocketed my abilities.” Additionally, as we walked into the bullfight, Jennifer told me (in English) that her Spanish had improved so much that people thought she was a native speaker. Regarding that I wrote in my field notes:

I should point out that she struggled in any tense other than the present and that she has a strong American accent. I should also note that my skepticism is due to the fact that Spaniard males tend to have exoticized views of American women, it is the festival, and Jennifer is a pretty, twenty year old.

Nevertheless, both her capacity to comprehend and to speak (in the present tense) grew significantly on this trip.

Jennifer expressed one regret regarding Spanish learning; she wished that several of her fellow students had been willing to speak Spanish when they were outside of classes rather than English. She stated that she believed if the study-abroad program had put the PSU students in classes with Spanish students, or even with other European students that they did not know, she could have improved even more linguistically. I 
wrote later in my field notes that this may be one area for Dr. Green's program to improve Spanish outcomes.

More importantly, however, for this dissertation, was the socio-cultural outcome of Jennifer's initial struggle to understand Spanish. She explained to me in the exit interview (and confirmed in the member-check interview) that her difficulty in understanding Spanish and feeling "foolish" to try to meet Spanish young adults had made her much more empathetic to English learners in the United States. She stated, "I mean it definitely gives you a different perception.... It allows me to see from their perspective what it feels like to ... have everyone know you're different." In the member-check interview she explained that the experience of being linguistically "othered" in Spain made:

me a lot more patient with [people learning English].... I actually like having people who don't speak English ... asking me for help, because ... if you don't speak my language, then let me help you understand it.... I think [living in Spain] gave me a much broader perspective."

Jennifer went on to explain that previous to the study-abroad program, she "did not have a lot of patience" with English learners.

Jennifer's experiences with personal linguistic deficiencies and her subsequent socio-cultural transformation in attitude about English learners in the United States echoes Foster's (1997) findings about the anxiety or vulnerability associated with learning another language. According to Foster (1997), this vulnerability sets up an excellent situation for transformative learning to occur because learning to communicate 
in a different language is unsettling, causing a distorted view of self. Furthermore the risk associated with learning another language requires the learner to relinquish her or his view as a competent communicator in the native language. This risk was especially challenging to Jennifer given her self-perception as an excellent student and natural disposition to be participative in class and social discussions. This marginalization appeared to lead to authentic subjective reframing where she saw how her own previous actions had been discriminatory and she chose to act in a more empathetic way (Mezirow, 1998). Jennifer's transformation from perceived linguistic “othering” also aligns with Johnson-Bailey and Alfred's (2006) theory that marginalization is a powerful socio-cultural transformation catalyst.

What is each student's racial, economic, gender, and cultural identity? How does each student's identity impact her/his worldview and her/his transformations (or lack thereof) during the study-abroad program? As mentioned in the introduction, Jennifer was a twenty-year-old student in between her sophomore and junior years of college. Jennifer lived with both of her parents before going off to college and had close relationships with both of them. Her mom, often "pushed [her] to do the things [she] needed to do in life" despite being the self-described "world's worst procrastinator." One thing Jennifer missed about being in the United States was her close relationship with her dad (loving, talkative) compared to her relationship than her host dad (distant).

Class privilege. Jennifer was from an upper-middle class home in a racially, culturally, and economically diverse city that was geographically distant (several hours) 
from the rest of the participants' hometowns. However, some of her comments conveyed that she had not been exposed to as much diversity as she felt she had been. For example, Jennifer told Juan, "The fact that everyone lives in apartments shocked [me] because almost everyone has houses in the US.” Additionally, Jennifer showed cultural insensitivity several times telling teachers and LU staff she participated in the studyabroad program "to get a tan." Additionally, explaining her reactions to an in-class discussion on the post-2008 economic crisis in Spain, Jennifer told me in the exit interview she felt Spaniards handled difficult financial times better than Americans. She said, "they have a $25 \%$ unemployment rate, and, yes, there's [sic] beggars everywhere, there's [sic] homeless people everywhere, but they don't ... act like their life is over [like unemployed people in the United States]." Furthermore, she informed me during the member-check interview that she came on the study-abroad trip primarily because her good friend Kristene was doing it and she thought it would be fun to do as well. Few individuals share the economic capacity to do something as financially intensive as a study-abroad program in Europe.

Other discussions indicated she was accustomed to be the dominant voice. For example, I came into class one day to hear Jennifer teaching Juan how the Spanish zeta ([$\theta]$, unvoiced, interdental fricative) sounded weird to her compared to the ese ([s], unvoiced, alveolar fricative) of Latin-American Spanish. I wrote in my field notes, “Jennifer was trying to teach Juan that the zeta doesn't exist in the Americas (as if Juan did not know)."

Race and class privilege. Jennifer often voiced complaints about situations that 
did not have the outcomes she desired. While expressing complaints was common to almost all of the participants, I observed Jennifer repeatedly place blame on external, institutional forces such as Dr. Green, the LU program, or on Spain/Spaniards anytime something did not have the outcome she desired. I never saw Jennifer proactively seek to change situations that were not in her favor. Nevertheless, she demanded that the studyabroad program eradicate her concerns rather than providing herself possible solutions. I postulate from observations and interviews with Jennifer that this expectation (which was not expressed by students of lower socio-economic class status) was grounded in Jennifer's racial and class privilege.

Jennifer's search for water provides an example of her expectation that it was the program's duty to provide positive outcomes for her. In the entrance interview she told me, "I constantly drink water ... but [what my host family provides is] never enough." At the first focus-group meeting, Jennifer told Dr. Green that she could never get enough water at her host family's flat (because of a lack of fresh water in the area, Levante's tap water was half fresh water and half salt water from the Mediterranean, so bottled water was required). Dr. Green encouraged her to either discuss the situation with her host family or buy some water for herself. He explained that the host families were only required to provide them breakfast and supper each day and that any additional food or beverages were not the host family's fiscal responsibility. Because I was concerned for her physical health (and because she was sitting next to me in the focus-group meeting) I offered a suggestion while Dr. Green went on to another subject. Below is an excerpt from my field notes: 
Jennifer said that she can never get enough water here. I was sitting next to her and said, "if you go to the supermarket, it will only cost you .60 Euros for a 5L bottle, when I bet you are paying 1 Euro for little bottles that are $200-500 \mathrm{ml}$. She told me that there are no markets near her house. I told her about the little convenience stores that are [every block or two]. She could then use one of her $.5 \mathrm{~L}$ bottles as a water bottle. She told me that there are no stores, no convenient stores, or anything like that by her house. I assured her that if she went out exploring she would at least find a convenience store. Dr. Green went down to [the housing coordinator] to explain the situation [after the focus-group meeting.] I told her my story and she said that [Jennifer's] host family lives less than one block from the Mercado Central [the biggest fresh food market in the city of Levante] and just a couple blocks from Mercadona [the biggest grocery store in Levante]. I'm still sure there are probably 4-5 convenient stores in the two-block walk to Mercadona, as well.

After Jennifer found out that Dr. Green had talked to the housing coordinator, and that she had called Jennifer's host-family to ask them to make sure she had enough access to water, Jennifer was upset with Dr. Green for making her "look bad" with her host family. Several other examples serve to further illustrate this institution-blaming phenomenon. When Jennifer and another student arrived late on the first day of classes, she claimed the map incorrectly placed the building where classes were held on the other side of the library. During the focus group interview, she listed a series of complaints so long that we had to continue the interview on public transportation. She started by 
saying, "I've only been here for two weeks, and I can honestly say that I think the system is extremely flawed. I would make many, many changes if I could.” Her first criticism was that the study-abroad program was "messed up" because she did not feel she was meeting enough Spanish students her age. She argued, “you don’t put your group of eight students in one class together the entire time we're here. Integrate us with other people in the University." Another concern was that her host mom ruined one of her shirts. According to her, when she approached Dr. Green about the situation, he said, "Sorry, that's the culture" and encouraged her to discuss the situation with her host mom. In Dr. Green's defense, when he previously intervened by having the housing coordinator call Jennifer's host mom about her lack of water (he was concerned for and responsible for her physical well-being), Jennifer maintained that Dr. Green damaged her relationship with her host mom. She also claimed that Dr. Green did not prepare the students enough for cultural clothing expectations. When I interviewed Dr. Green about this later he informed me he had a series of monthly presentations to prepare the students and appropriate clothing was one of the topics. She also felt the group did not do enough cultural excursions. Yet, regarding the cultural excursions they did she stated, "You can only go to the same place and walk around the same area so many times." Similarly, when Juan informed the students that the bus schedule would change for the festival, several of them stated that they did not know where the new stop was and did not want to walk that far, even though it was about a 5 minute walk (500 meters), from their other stop. Ironically, the new stop was the Mercado Central where Jennifer did not go to search out water. 
Although Jennifer's transformations were sincere and personally relevant, I have no indication from the data collected that they involve critical reflection on how her personal worldview impacts others. The world, her fears, and her view of other nations' political structures authentically became "smaller" and manageable in K. Taylor and Elias' (2012) view of subjective reframing. However, from all indications, Jennifer's liberalism is the kind that CRT scholars critique as saturated in the myths of colorblindness and the meritocracy (Taylor, 2009). The one exception, of course, would be her desire to be more helpful to English learners. While I do not downplay the importance of this transformation and the impact it may have on society, even her desire of "let me help you" carries with it a form of privilege and superiority often criticized as White saviorism in CRT scholarship (Gambrell \& Bright, in press).

Her Mezirow-styled (2012) personal transformations also do not align with CurryStevens' (2007) call for pedagogy of the privileged within travel for transformation wherein individuals with race and class privilege develop a "counterhegemonic ... framework of praxis, whereby ... transformation is linked with becoming an ally in struggles for justice" (p. 34). Rather, her worldview appears to be saturated with racial and class hegemony experienced by people of color and other marginalized groups, yet remain invisible to individuals in positions of societal privilege (Leonardo, 2009; Tatum, 2009). Likewise, Brah and Phoenix's (2009) CRT critique of whiteness cited in the literature review applies to Jennifer's transformation lacking a self-critical, socio-cultural stance. They state, "people do not generally see the ways in which they are privileged, 
and so well-intentioned, middle-class, white liberals often strive to maintain privilege ... while denying they are doing so" (Brah \& Phoenix, 2009, p. 253).

I posit that Jennifer genuinely believes in liberal values such as marriage equality and racial and gender equity (she actively advocated for these values in the LU classes). Furthermore, it is important to note that I observed that rather than active discrimination, Jennifer's views are a reproduction of liberal media and textbooks that "conjure up images of domination happening behind the backs of whites, rather than on the backs of people of color," in which domination is removed from agents of oppression (Leonardo, 2009, p. 262). It is also important to note that the study-abroad program may be a catalyst for future transformation toward critical self-reflection of how Jennifer's frame of reference impacts the socio-cultural norms she deeply values. The critique of her epistemology is tied to the limitation of the data gathered for this study, not in lack of hope for her potential to critically reflect and socially act in the future (Tatum, 2009). Indeed, Jennifer's limitless potential for personal growth that is at the root of TLT's humanist axiology (Cranton \& Taylor, 2012), combined with Jennifer's liberal values, offer me optimism that this study-abroad program is simply one point in a possible series of incremental transformative opportunities for critical self-reflection Jennifer may have throughout her life.

\section{Participant Three—“Anne"-Social Marginalization and the Chrysalis of Personal}

\section{Liberation}

Anne was twenty-four years old at the time of the study-abroad program. She was in between her junior and senior years of an undergraduate Spanish degree. Although 
Anne had traveled around much of the Southeastern, Midwestern, and Rocky Mountain regions of the United States, she had never been outside of the United States before the study-abroad program. After explaining that her mom had been to Europe before, so she said, "I'm graduating in December, so I thought, 'Why not take a little trip and have a little fun and take some classes to work toward my ultimate goal of graduation?"' Furthermore, Anne believed "it's extremely important if you learn a language, you should go somewhere where they speak the language so you're fully immersed in every aspect of it."

Anne was from the most-wealthy county in her Midwestern state. I thought that Anne was a middle class student raised in an affluent suburb of a major city during the study-abroad program. What brought me to that conclusion was that several times she mentioned being "poor," yet her speech and worldview were saturated in whiteness (Tatum, 2009), the myth of the meritocracy (Stovall, 2013a), and deficit viewpoints (Bell, Jr., 2009) of poor individuals and people of color discussed in the CRT section of the literature review. However, in the member-check interview, she corrected me by telling me that her family was easily upper-middle class. She said, "I was raised in such away that I just don't act like it. And I would also say that my family's more upper-middleclass... I've gotten everything I've ever asked for. There is nothing I have never gotten." Anne's parents paid for her study-abroad program and her grandparents gave her some money to use as spending money for the trip. Before college and after graduating, Anne lived with both of her parents. Anne's class privilege will be discussed in further detail in the final section of her findings. 
As a researcher, I struggled getting to know the real Anne. There were times that she manifested, in her words, a "Type-A personality." However, it was also evident from her comments that she struggled as a student. Anne told me in an informal interview that she almost did not graduate due to her low grade point average. She needed to get at least one "A" and a "B" in the study-abroad program to graduate. As an observer (informed from the viewpoints of Anne's peers and teachers), I would call her "intense," rather than "Type-A." In the member-check interview, she was excited that she had "only had one panic attack the whole time in Spain," which she saw as extraordinary. In addition, one of her fellow study-abroad participants labeled her "more of a drama queen than a type-A personality." Throughout the study-abroad program, Anne became visible angry or frustrated when she did not achieve the outcome she desired. Furthermore, she was quick to complain to her peers. However, in contrast to Jennifer, who was transparent with her emotions with authority figures, Anne often said what she thought they wanted to hear. I wrote in my field notes during the Alhambra cultural excursion: Whenever Anne thinks someone is not listening to her, I hear her complaining. When she knows one of us (Dr. Green or I) is listening, she is having the time of her life. I do not know how to address this or how to get to the real person. We all complain, that is not bad, especially for my dissertation [which discusses culture shocks]. However, when she actually talks "on the record" I get different information.... What I don't know is what Anne is the "real" Anne. Is she the unimpressed suburbanite? Is she just telling me what she wants me to hear, what the teachers want her to hear? Is she a student who is 
allowing the culture of Spain to permeate her like she says? Is she all of those? After analyzing data regarding Anne, I posited that she was a complex person, with multiple aspects that came out at different times. She confirmed this finding in the member-check interview. She stated:

I think that's very fair. Because ... I just felt like I was experiencing way different things week-to-week.... The first week you saw me, and I was very Type-A. I wanted it this way, and it wasn't good enough if it wasn't.... And then, week three, I complained a lot. ... I think I just... had a lot of time to think about things. So week-to-week, I was just going through different aspects of my life.” I must take some responsibility for Anne's reticence to open up to me as a researcher, however. During the entrance interview, Anne informed me that she was raised as an only child. Despite being raised as the only child, Anne had a huge, closeknit extended family, so she felt that she had the benefits of siblings because she and her cousins were close and they spent a lot of time together.

A few days later in class, Juan was explaining that most Spanish millennial children are sole children. He said, "I bet none of you are from one or two children homes. Anyone?" I was sitting behind Anne, and I whispered, “Anne." She did not respond. However, in between classes she took me out in the hall and said, accusingly, "I thought everything that I say to you is confidential!" I told her that, indeed, it was. She said, "It embarrasses me to be an only child!" And then she broke down in tears. Previous to this conversation, I had never considered that the "get-to-know-you" questions of family make-up might be sensitive information, especially since it was all 
said loudly on a bus with all of her classmates within earshot. Additionally, I don't think any of her classmates heard me whisper, "Anne." But she was absolutely right—I had broken confidentiality and betrayed her as a researcher.

After I apologized profusely and promised to better maintain confidentiality, Anne explained that her father had been married before her mother. She had a halfbrother, with whom she had almost no relationship. She did not like explaining this to people, because she felt like she was raised as an only child, and it hurt her to discuss her half-brother. Her half-brother was alienated from her father and had lived a difficult life of addiction and depression, for which Anne experienced an acute sense of guilt. In the member check interview, talking about her father's relationship with her half-brother, Anne explained:

You're gonna spoil the hell outta me, but you're not gonna pay any attention to him whatsoever? ... If your child is ... cry[ing] out for help with alcohol, you should probably fucking listen. And he just is like, "Eh, whatever!” ... I love my father ... but there are days when I'm just like, "What the fuck is wrong with you?” “

This guilt was so intense that she developed "a habit of drinking in her closet" to hide the pain. She also had frequent panic attacks and high blood pressure related to her guilt. She later overcame the drinking habit, but never overcame her guilt of living a relatively charmed life compared with her half-brother.

As I came to know Anne better in weeks two and three, I further discovered that she was slow to warm up because she had previous complicated interpersonal 
relationships with men (in contrast, Anne spoke extremely highly of her mother). In the member-check interview, she told me, "I was abused by my first boyfriend mentally and emotionally... so that really hinders my ability to trust ... and care about people.” Additionally, she was physically and verbally abused by her fiancé with whom she terminated their engagement just before the study-abroad program. Lastly, she described her relationship with her father as "complicated," but would not go into any further detail saying, "My whole life I have been really taught to repress things, and you don't talk about it. And if it makes my parents feel uncomfortable, we don't talk about it." Anne's personal silencing aligns with Mezirow's (1998) findings that silence, especially being silenced, reinforces oppressive practices (see also hooks, 1994). Furthermore, Anne's silence parallels Tatum's (2009) postulation that guilt reifies oppression, while awareness leads to hope and liberation.

About talking with anyone about her difficult past, especially a male, Anne explained, "I was really, really skeptical. So, ... I would give you little bits, but I would never give you ... everything, until about, probably the beginning of week three." I will discuss the limitation of my maleness as a limiting factor in retrieving full information from Anne and other female participants more completely in the Discussion chapter.

To what extent do seven students experience the elements of transformative learning during a month-long study-abroad experience in Spain? Anne exhibited multiple experiences with culture shock and cognitive dissonance during the studyabroad program. She also experienced both an incremental transformation as well as an epochal transformation. I will also discuss how Anne's time for reflection added to 
opportunities for . In addition, she experienced both cultural and linguistic "othering" in the ...which align with both Morgan's (2010) findings that cultural and linguistic "otherness" can create cognitive dissonance that leads to transformative learning. Finally, I will discuss Anne's biggest transformation of liberation from abuse and guilt, which matches Mezirow's (2012) transformative learning (Mezirow, 2012). However, even in her transformational learning, Anne displayed both racial and class privilege, which will be discussed in the last subsection of Anne's findings (Johnson-Bailey, 2012).

Cognitive dissonance. In the following section, I will discuss culture shocks in which Anne experienced cognitive dissonance. Adjusting to the pace of life in Spain, homesickness, and the bullfight where her biggest (and repeated) experiences with cognitive dissonance. Navigating airports, nudity on the beaches, and other minor examples of cognitive dissonance (which I will label cultural adjustments) are discussed at the end of this section. Finally, Anne experienced some positive cultural surprises from the festivals, political diversity, and a change in eating habits.

Culture shock: Scheduling and pace of life in Spain. Anne had a difficult time adjusting to host family, university, and public transportation scheduling during the first week of classes. She was visibly and verbally frustrated when she arrived over a half hour late the first day of classes. Anne told Kynzee (discussed later) that her host "mom" told her that the tram (light rail) would only take fifteen minutes, but it took twenty-five minutes. Later in an informal interview, Anne reflected on the experience, "I didn't know that the tram was 30 minutes. Nobody ... told me that.... It stresses me out when I'm late, it stresses me out when I don't know where I'm going. So the two of those 
combined? I was just a hot mess." The second day of classes, she was about a half hour late again. This time, she expressed frustration because her host "mom" took too long making her a sandwich. According to the LU housing coordinator, because Anne had food allergies she was placed with the host family who had the most experience with hosting students with food allergies. I wrote in my field notes after the second day of classes, "There seems to be a lot of dissonance between Anne and her host family." Additionally, I overheard a conversation between Dr. Green and the LU housing coordinator. The coordinator called Anne's host "mom" after the first day and the host "mom" maintained she tried repeatedly to get Anne to leave earlier, but she would not leave on time either day. Anne maintained in an informal interview that the LU staff, Dr. Green, or her host family should have provided more support finding her way around and getting to know the city and how to get places. She stated throughout an informal interview that she felt "angry," "lost," "disoriented," "confused," and "alone."

As I got to know Anne better, I believe this was more about her perception of time in contrast to the Spanish cultural perception of time. Anne explained it this way in the focus-group interview:

I need to have a routine. And if that routine is broken ... Lord help anybody around me, 'cause it's not fun. So once ... I establish myself in a routine ... I'm a lot more comfortable, and I'm a lot more accustomed to what's happening, and I can just kinda go with the flow.

As time went on, Anne seemed to become aware that relying on public transportation and service from a host family simply takes more time. A week later in the entrance 
interview, she said:

The biggest thing for me is people are not in a hurry here. And, I am a very typical type-A [person]. [In the] United States, I work at a very ... quick pace. I'm not really used to taking my time with a lot of things. And, if I'm late, I'm very anxious. I get very flustered quickly, and I get frustrated because ... [Spaniards] are a couple steps behind me ... it's difficult to get used to that. As Anne looked back on the study-abroad experience and her initial struggles with the pace of life in Spain, she had a mini-transformation:

But once I figured it out and once I got used to [the slower pace], I actually kind of enjoyed it. And I still operate like that. Like now, after being home for however long, I operate on a much slower pace.... Like, if I am late, whatever. Fine. I don't care.

In the member-check interview, Anne said that after the study-abroad schedule she is "a lot more able to go with the flow" (which was a phrase she used in the exit interview, as well). Moreover, when I asked her what advice she would give a future study-abroad student, Anne counseled, "Stay calm. It will get better. It may not seem like it's very much fun at the beginning, but by the time it's over you will wish that you could do it again.”

Culture shock: Homesickness. In the entrance interview, Anne mentioned that she missed her lifestyle in the United States. She stated that she missed her pets, house, and a yard. About homesickness, Anne said: 
I live on the seventh floor of an apartment building [in Spain], so that's kinda been an adjustment, just because I live in a house in the U.S. And, I have a yard, and I have 2 dogs, and, not having pets ... is another thing that just is odd to me. I just grew up with animals in my house, but to each their own.

In the member-check interview, I asked Anne what she noticed when she first arrived in the United States after the study-abroad program. She stated:

The first couple of days after I got home were awesome. I got to see my dogs. And my dog was ridiculously happy to see me.... And I loved telling [my parents] about my trip and giving them presents ... especially my mom.

Culture shock: Bullfight. Anne was the most vocal of all the participants during the bullfight about her hatred of the practice. She did not seem disgusted by killing the animals like Kristene, nor did she seem politically charged like Jennifer. In contrast, Anne seemed angry. Anne had close family that owned a cattle farm. I asked her asked her if it made a difference that bulls for bullfights are typically grass fed, free range cattle (in contrast to the United States). She said that her family picks certain cows to be pets, and she saw her pets out there getting brutally killed. She informed me that the way cattle die in the United States "is much more kind" and that she hoped "the bulls win." Although Anne was strongly opposed to the killing of the bulls, she chose not to leave with Kristene and Jennifer at the intermission when Dr. Green said that they were welcome to leave if they wanted.

In the exit interview we discussed the bullfight. I asked Anne if there was an equivalent in the United States to bullfighting. She said that she felt NASCAR racing 
was similar in the hope of violent outcomes and the social class of the spectators. She also said, "NASCAR drivers get all decked out whenever they're going to a race, and ... going into it, the matador could possibly die, and so could the NASCAR driver, if they have an accident."

Culture shock: Spanish dislike of Americans. Anne was shocked at how much "the Spanish hate Americans." This will be discussed in the Cultural "Otherness" subsection below.

Cultural adjustment: Navigating airports. Anne was in the same travel group as Kristene and Jennifer on their flights to the study-abroad program. In the entrance interview, Anne noted the same difficulties in figuring out customs, "the Madrid Airport was ... like a maze. We really weren't aware that for non-European Union countries you have to go to a separate baggage claim." Furthermore, in the exit interview she reiterated, "There wasn't really a clear direction of non-EU countries here and EU countries here." However, in contrast to Jennifer and Kristene, Anne saw this as a chance to practice Spanish. In the member-check interview, she said:

You know it really did not surprise me as much as I thought it would.... When Jennifer, and Becky [discussed later], and I were at the airport. Everybody was like who's going to go and talk to these people? ... So, I just went up. Because you know very well I'm not really a shy person.... I just went up and started talking to somebody and was like, "Hey, where do we need to go for this? What do we need to do here? Where is that?" So that really wasn't ... a big deal for me." 
Anne saw herself as the resolution of the disorientation rather than the victim of it. Even though her Spanish was similar to most of the other participants, Anne's (somewhat over) confidence in her Spanish capacity allowed her to escape much of the linguistic "otherness" that other participants experienced. Anne's experiences in the airports did not qualify as a disorienting dilemma or cognitive dissonance (Mezirow, 2000). Rather, the experience reinforced her worldview as a capable communicator in Spanish. Even though Anne discussed airports several times as a culture shock, I would include it in a list of cultural adjustments that did not cause a reordering of epistemological assumptions and causing a change in beliefs and behaviors (Mezirow, 2000).

Other cultural adjustments. Anne experienced several other minor cultural adjustments, as well. First, Anne stated in Juan's class that she thought people would be more religious. In addition, Anne explained that seeing topless women was a "huge shock" that made her "very uncomfortable," but she conceded that she got "uncomfortable easily." However, in the member-check interview she stated, "Once I [saw nudity] a bunch of times ... it [got] more acceptable."

Cultural surprise: Festivals. Similar to other students previously discussed, Anne also experienced positive culture shock, which we decided to label cultural surprises. Like Jennifer, she loved the festival that happened the last weekend to the study-abroad program. In the exit interview Anne said, "I really, really, really enjoyed the [festival]." A few days before the festivals, Anne told me she couldn't wait to "get the fuck out of here!" However, after five days of festivals, Anne said, "I'm sad to go. I'm not ready." I postulate, as will be discussed below in the Cultural Inclusion subsection, that part of her 
change of mind regarding Spain was being included in Tess's (discussed later) and Paco’s (Tess's boyfriend/host “brother”) festival plans.

Cultural surprise: Political diversity. Anne also enjoyed learning about multiple dimensions of Spanish politics in Juan's class and found it exciting that there were more than two choices. She said in the exit interview:

I don't really look at other types of government as being "bad" or "good." ... I mean there are just different political systems... I feel like Spain is really...trying... to become even more modern than they originally ... were. And just the whole debate, "Do we have a king? Do we not have a king?" ... is really kinda cool, because everybody's allowed to have an opinion. In America, you just have this side, or that side, and you have to pick the lesser of two evils. And they're more willing to listen.

For historical context, it is important to note that politics were a popular topic both inside and out of class during the study-abroad program because King Juan Carlos, who had been king for 39 years abdicated the throne to his son Felipe VI. In the member-check interview, Anne reiterated this fascination:

There's (sic) just so many more layers of politics ... in Spain. And I guess... I knew that [previous to the study-abroad program], but I just didn't really realize how big a deal that was. So, I feel like that kind of surprised me.

Cultural surprise and mini-transformation: Eating habits. Anne also discussed in the member-check interview that she "really noticed a change [her] eating habits." Furthermore, she said, "I don't like salad dressing anymore. I have to eat it with olive 
oil." Additionally, she critiqued the college town for the shortage of healthy eating opportunities, "I've noticed that I want to eat more healthy things, but there's really not as much here as there is [in Spain]."

Transformations. I observed that Anne experienced two structural shifts in how she saw the world and acted in it (O'Sullivan, 2012). Anne experienced an incremental transformation in how she saw herself as a capable student and an epochal transformation in a change in understanding power relations or structures of gender, which led her to a more autonomous use of personal agency (Cranton \& Taylor, 2012; Ettling, 2006; Mezirow, 2000; O'Sullivan, 2012; Taylor \& Elias, 2012). I posit that both of Anne's transformations align to Tisdell's (2012) requirements that a transformation should lead individuals to live more deeply, experience hope, and desire to act emancipate themselves from unsustainable attitudes and behaviors (Tisdell, 2012). Although it is not observable from the data collected, Anne's epochal transformation wherein she rejected former feelings of guilt associated with abuse from men could be a life-changing transformation with long-lasting effects.

Incremental transformation: Confidence as student. As I analyzed Anne's data, I repeatedly noticed that she did not speak in class unless a professor directed a question to her or she was required to speak in a presentation. In presentations, she was well prepared. When called upon to read, her Spanish was as intelligible as any of the other students, excluding Tess (discussed later) and Kristene. I began to question whether Anne was a quiet student or was quiet because of limited Spanish proficiency. I asked her why she did not speak much in the classes during the member-check interview. She 
said, "It's not just a Spanish thing... I was in a very new situation. And when I'm in a very new situation, I usually don't talk very much.” She further maintained that she was not shy, but reserved (my observations concur). While Anne was very affable in interpersonal conversations, she simply did not talk much in class.

As we talked about her reticence to speak in class, she said that she had to be very focused during the study-abroad semester because she needed "As" or "Bs" in order to graduate from college. Furthermore, the study-abroad program to Spain "symbolized the end [of my major] for me. And it made me strive to achieve so much more then I was able to do before." Anne proudly informed me that the semester before the study-abroad program, she earned her "first-ever 3.0" grade point average. During the study-abroad program, she received a 3.6 grade-point average. The semester following, she had two “As," a "B," and a "C." Anne maintained that working hard to achieve something her parents had promised to pay for if she was on track to graduate: change[d] the way that I did school. And it allow[ed] me to be a little more proud of myself... Not only did I go to school during this experience, but I left home, and left the country, and left mom and dad to do it. Consequently, Anne felt that she could succeed as a student, which was not a self-perception she had before the study-abroad program.

Anne's change in self-perception aligns with Morgan's findings that willingness to be changed by the travel experience increases when there is a purpose behind the travel like study abroad (Morgan, 2010). Anne felt that this program was the culmination of everything she had worked for throughout high school and college. In addition, Brown's 
research found prolonged absence from a student's home culture could help students revision or reframe their professional and domestic roles. Although this study-abroad program was only a month instead of a year like Brown's study, it appears that the duration sufficient to separate Anne from negative external stimuli in her home and romantic relationships in so she could perceive herself as a competent student.

Epochal transformation: Room as chrysalis. Like mentioned above, Anne was reserved, but not shy. My error of accidentally revealing her only child status made her even more guarded than she already was. However, by the end of the second week of classes, she talked freely and often with me. Moreover, she tended to be a lot more talkative in interpersonal, rather than group settings. Interestingly, as I analyzed the data, I observed that more than any other student, Anne spent much of the time in her room at her host-family's house. Many of the other participants liked beach-related activities, yet she had red hair and light skin, making activities in the sun painful. Although Anne traveled with Kristene, Jennifer, and Becky (discussed later), she was not really ever an integral part of the group. It was not that the other participants disliked Anne. Rather, I observed they were accustomed to her excuses and were not willing complicate their planning by trying to work around her mood swings and panic attacks. The group seemed to like her personally, but did not tolerate the complications of planning around her.

The final dinner of the study-abroad program serves an illustration Anne's planning complications and reclusion. Anne did not show up at the meeting place for the final dinner. Dr. Green was concerned and asked the other students to call her. Anne said that she did not feel well and was not going to come. Several of the participants 
rolled their eyes as Dr. Green tried to convince Anne to come to the dinner. Several of the students told him that she was "in one of her moods." Consequently, Anne missed the final dinner, which was an elegant feast and a chance for reflection upon all that the participants had learned and experienced over the month in Spain.

Additionally, in Anne's host family's house, the "parents," "siblings," and the other study-abroad student never really accepted her. Consequently, Anne spent much of her free time not in classes in her room. However lonely this may sound, I came to see Anne's room as a chrysalis of personal liberation — a place where she rested, recharged, and grew. Anne's view of her past relationships with men and her place in the world changed dramatically even though she often appeared like she was not a full participant in the study-abroad program or in the social activities of the other participants.

Because Anne was discussing a problem with her host family situation with Dr. Green during the focus-group meeting, I interviewed Anne the following day to discuss some of the themes and transformations that she saw in herself about half-way through the study-abroad program. In this interview, Anne said that the study-abroad program had given her time to "get to know herself." Anne mentioned that this provided her with reflection time, which, in turn, led her to liberate herself from guilt of past relationships, work, and family problems. She stated in the member-check interview:

Being alone, and being forced to be by yourself, and somewhere where you really don't know anyone, and you don't live with other people who speak your same language... you just ... have a lot of time to reflect and ... think about your life and the way you see things. 
On a similar note, Anne discussed her time spent reflecting in the exit interview: I've really had a lot of time to think about the way I view things ... [and] my perspective on things.... I've noticed ... I stand up for myself a lot more. I'm a lot less willing to take people's crap. And I ... don't necessarily have to blame myself for every single thing that ever happens.

Previous to the study-abroad program, Anne additionally experienced guilt for not having a relationship with her half-brother. However, during this time of reflection, Anne came to the conclusion that it was not her fault that her half-brother refused to have a relationship with her or her family. She explained during the exit interview:

My dad and his ex-wife and my half-brother ... that's not my problem. That's not my fault either. Because, I really didn't ask to be here, I didn't ask for my parents to create the thing that is me.... Ultimately I'm the sperm that won, and that's it.... For a very long time, I really did feel like it was my fault that my brother hates my dad and that he hates me.

Another positive outcome of the added time for reflection was Anne came to understand that her abusive relationship with her ex-fiancé was not her fault, either. She told me that he was a physically, verbally, and emotionally abusive alcoholic. She maintained that she had given back her engagement ring a month before the study-abroad program. The reflection allowed her space to "make peace within my own mind about things.... It just kind of snowballed and all the things that I had been running away from forever ... caught up with me." When discussing the reflection time and this personal epiphany, she explained in the exit interview: 
I may have been abused, and, yes, I chose to date that person, but did I ultimately know that that person was going to do that to me? No. He was one person at the beginning of it, and then, four months later, turned into a complete nightmare. Moreover, Anne often skirted the issue, but from her comments, it was evident that her relationship with her father was fragile and she had found similar patterns in dating that she had grown up with. Nevertheless, during the time of reflection, Anne came to understand that she deserved more. She wanted to be treated with respect and dignity in all her relationships. She said:

My dad actually told me that [dating him] was my fault, and that I asked [to be abused]. My own dad would say that to me.... I realized that as much as my dad hurt my feelings, he's completely and totally wrong. That's not my fault, I didn't ask for that. Nobody asks for that, because I'm pretty sure if I was able to see into the future and know that that would be happening to me, I can guarantee you I wouldn't have made that decision [to date the abusive fiancé].

During the Alhambra excursion, I wrote in my field notes that I was having a hard time getting to know the "real" Anne. However, by the beginning of the third week, I felt like I had gained Anne's trust and build rapport with her (which she confirmed in the member-check interview). After an interview at the beginning of the third week, I wrote: [Anne] is an injured soul. The complaining and criticism are indicative of her inner personal struggles rather than a socio-economic statement. I also think some of the things I saw in the beginning as extreme, upper-class bias are more a re-production of those upper-middle class values/biases/prejudices that 
surrounded her upbringing in schools, friends, and family.

When I followed up on her liberating experiences in her "chrysalis" during the member-check interview, I commented that the reflection time seemed to be empowering as a student, as a woman, and as a human being. Anne agreed, "It really was. And it's funny because I was kind of talking to someone while I was there. Like we just started talking, and now we've been dating forever." Because of the study-abroad program and because of her freedom from irrational guilt, Anne said, "I feel like now I'm a lot more able to open up into be emotional and not feel guilty or bad about it." She told me that she was in the first healthy romantic relationship ever.

In the literature review, I described transformative learning as a means to reframe untenable worldviews through critical reflection on the assumptions upon which an individual's interpretations, beliefs, and habits of mind are based. Anne appears to have undergone what Ettling (2006) described as a "deeply engaged process" (p. 63) of relinquishing assumptions that were previous ways of unjustly coping with the negative behaviors of others. Anne seemed to move toward a more liberated view of herself as she sat in a small room removed from the negative experiences of her past. Indeed, she appeared to arrive at a "more autonomous sense of self characterized by agency, choice, reflection, and rationality" (Taylor \& Elias, 2012, p. 150).

Anne's liberation-from-guilt transformation seemed tied to critical reflection of self assumptions about her place in society, as Mezirow (1998) described. However, Anne's experiences more closely align to Johnson-Bailey and Alfred's (2006; see also, Johnson-Bailey, 2012) conclusion that marginalization may be a powerful transformer 
that leads to, or even replaces the need for, critical reflection. Furthermore, Anne's transformation reaffirms recent empirical research that concludes that reflective discourse and a skilled educator may not be as effective in transforming frames of reference as experiences are (Baumgartner, 2012). Moreover, Anne's huge shift in epistemology parallels Ross's (2010) conclusions that transformation in travel is more likely to occur when space for reflection is given through activities that stimulate contemplation about the traveler's experiences.

Anne experienced subjective reframing, which stimulates critical reflection of one's epistemological underpinnings and examination of the reasons for limited frames of reference. She really experienced a disorienting dilemma in that something had to change. According to TLT, Anne could not continue to justify her negative self-opinions and positively contribute to the world (Mezirow, 2000). However, critical reflection of beliefs ideally leads a person to emancipatory action, making the person whose worldview has transformed aware of the possibility to be an agent for change (Baumgartner, 2012; Merriam \& Kim, 2012). Thus, while Anne's subjective transformation was deeply engaging and important on a personal level, it did not appear to motivate her toward more justified beliefs or action toward others, which will be discussed in greater detail in the last subsection on race and class roles in Anne's findings below.

What role did encounters with cultural and linguistic "otherness" play in the transformative learning (or lack thereof) of each participant? As mentioned earlier, Anne's red hair and light eyes immediately gave her away as "other" in Spain. When 
talking about how easily identifiable she was as a foreigner in Spain, Anne said, "It's just because of my hair. That's the only reason. And my eyes are a weird color, than, you know, everybody's brown eyes here." In the member-check interview, she said that she felt her red hair and light skin tone caused a man to come up and start kissing the back of her neck during the festivals. She said it must've been because she was a "tourist" and because of her "red hair and freckles." One thing that irritated Anne was that "People [were] always constantly asking me, 'Where are you from?' 'What are you doing here?'” Then, very disdainfully she said, "I'm from the U.S." I start this section with this piece of demographic information, because Anne was easily the most physically distinguishable as "other" based on her physical characteristics alone. However, throughout this section I will discuss other ways in which she experienced both cultural and linguistic discrimination.

Cultural "otherness:" Isolation. Anne was essentially alone, even when she was in a group of people. As discussed earlier, she felt socially marginalized by the "family" members living in her flat. Additionally, like mentioned earlier, the group tolerated Anne, but she was never fully "part" of the group. This was something that both Anne and her closest acquaintances from PSU (Kristene, Jennifer, and Becky) indicated throughout the month. However, if the amount that she discussed it in interviews is to be considered, Anne seemed to feel more frustrated and angry from her alienation by her host "family." In the entrance interview, she said:

I have another foreign-exchange student living with me, and she's from France. We really haven't had that much interaction, just because we're both kind of in 
our rooms and kinda just doing whatever. And I'm pretty sure [my host "mom"] has two kids... I haven't really had much interaction with either of them, really. [My host "brother"] doesn't really talk to me, and [my host "sister"] will say "Hi”" in passing, and kinda talk to me a little bit, but not really very much. And then, my host-dad actually reminds me a lot of my own. So, I feel a little comforted because he's very quiet.... He'll talk and he'll ask me how my day was, but he just isn't really very super-talkative, and he just kinda watches TV and does what he wants.

In the exit interview, Anne explained about her difficult relationship with her host family by saying, "Nobody in my house really talk[ed] to me except for my 'mom' and my 'dad."' I should note that this was not a one-way isolation issue. Anne admitted that she did not make a huge effort to relate to her host "family," either. She related her daily exit routine from her hosts' flat in the member-check interview:

I accounted for when they go [to] take my shower and when I knew my host mom was at work so she wouldn't try to make me a sandwich and make me 19,000 minutes late. You know, just other stuff... and I wouldn't have to say goodbye and be all awkward and stuff and I would just leave. And I would look once and look twice and just be out the door.

Essentially, throughout the whole study-abroad program the issue that caused Anne the most concern was when her host "mom" chastised Anne for not having her dirty clothes in the correct place on laundry day. I could tell Anne was confused, but 
even more, she was upset because the "only person who talks to me" in the house was upset with her.

Anne told Dr. Green about the laundry experience, and according to both Anne and Dr. Green, he told her to go talk to her host mom about it and work out a possible solution. Subsequently, Anne felt that Dr. Green was disinterested in her experience in the program, as well. In the focus-group interview, she said, "I feel like, if you're bleeding or dying, then [Dr. Green] will help you. But...if limbs aren't gone and you're not needing multiple Band-Aids, he's just like, 'Alright, whatever.'”

As Dr. Green and I were walking away from the university that day, he told me that one of the study-abroad students had told him she couldn't wait until this program is over so she could go home. I did not want to betray his trust with the student, so I did not ask him who it was, but I had a strong suspicion it was Anne. In the member-check interview, I asked Anne for a recap of her stay. She said about this same time right after the focus-group interview (beginning of week three), "I hate this place. I can't wait to go home in a little bit," and a little while later, "I can't wait to get the fuck out of here!"

Like I stated earlier, Anne was allergic to wheat. The housing coordinator described Anne's host family as the "best" and "most experienced" family, in housing students with any type of special dietary or physical special need. Anne appreciated that her hosts new about gluten sensitivity and that they prepared special meals for her. In the member-check interview, Anne explained:

The only good thing about [my host family situation] is that they don't try and kill me with food. So... I guess you have to take the good and take the bad.... I 
would rather have things that I can eat rather than things I can't. If that means that the family really doesn't like me, well, okay. You know, they knew what they were getting into ... but I feel like ... they don't like me because I'm American.... I don't really know why.

The theme of Spanish hatred of Americans wove throughout the tapestry of Anne's data. For example, in the entrance interview she said:

It really surprised me how much Spanish people hate Americans. I sat next to a girl, just a random girl, on the tram to school the other day, and Kynzee and Jennifer ... and Tess were sitting ... there with us.... I didn't really see the look on her face because I was next to her, but they [said] ... she just was pissed off as soon as we sat next to her because, I mean, we're American.... You have stereotypes.... You believe about certain places.... It just kinda makes me feel weird that I would want to go somewhere where they hated people like me so much.... I just don't understand why they don't appreciate the effort, I guess. You know, I've been learning Spanish for 9 years, and to come here and have you be like, "Oh, well Americans this, Americans that, Americans are dumb" and whatever.... It's just something that I just kind of shrug it off.... I know ... I'm not a stereotype.... I make the effort.... Being a Spanish Interpreter is what I want to do, and ... I feel like this is one step closer to ... helping me accomplish that goal.

About the statement above, I wrote in my field notes, “Anne ... was shocked that people hate Americans.... I wonder how she will feel about this in a month. I wonder if she will 
come to see, as I did, why many countries do not like our imperialism and hubris." However, Anne never came to see during the study-abroad program why Spanish people might not like cultural and economic imperialism. Rather, she reiterated this confusion in the member-check interview and told me she never came to understand why someone would have such negative views about people who looked differently or were from another country. Later, in the Student Identity section, I will describe that Anne shared many of the discriminatory viewpoints about "others" in the United States that she felt while being "othered" in Spain.

Contrary to Johnson-Bailey's (2012) postulation that marginalization may be the best transformative educator, Anne's experiences with discrimination did not seem to motivate her to move toward more just ways of seeing the oppressed within United States' culture. Anne was capable of objective reframing of seeing how discrimination was unjust when Spaniards discriminated, but incapable of the subsequent subjective reframing necessary to break through her own unjust worldview (Mezirow, 1998). I wrote in the literature review that subjective reframing is much more difficult than objective reframing (Mezirow, 1998) because it compels an individual to look inward see how her or his beliefs lead to distorted, constrained, or discriminating ways of being (Brookfield, 1998, 2002). Moreover, Anne's lack of subjective reframing regarding discrimination based on social constructs of race and class during the data collection does not preclude it from happening in the future. Rather, Merriam (2004) argued that subjective reframing may be too difficult or too painful individuals are in their thirties or forties. 
Cultural inclusion: Tess and Paco. Tess (discussed later) and "Paco" (Tess's host "brother" and boyfriend) invited Anne to experience the festivals with them.

Because Paco was from Levante, he took Tess and many of the participants to events that local students, rather than tourists, enjoyed during the festivals. In five days, Anne's feeling about Levante changed. She informed me in the exit interview she "did not want to leave" and was "considering coming back to teach English for a year." Anne never went into detail about what events changed her opinions, but she did give the credit to feeling included by Tess and Paco (and a little bit of alcohol). I was shocked when she informed me in the exit interview that she "did not want to leave," because approximately a week earlier she said that she "couldn't wait to go home." When I expressed my shock, Anne said, "Trust me, it's a shock to me!"

No observed or stated linguistic “otherness.” From the extensive data I collected from Anne, she did not express or manifest experiencing linguistic "otherness" throughout the study-abroad program. Rather, I observed (and Dr. Green confirmed) that Anne had an inflated view of her capacity to speak and understand Spanish. Like most of the other participants, Anne could speak with few grammatical errors in the present tense and comprehend pretty well after the second week of the study-abroad program. Rather than noticing linguistic discrimination, Anne presupposed that it was her physical characteristics that gave her away as a North American. She said, "I mean I look like a tourist ... but that really doesn't matter to people. 'Cause I've had people [say], 'Oh, you speak very well.' I mean, everybody tells me that." In the member-check interview she reiterated: 
I think a lot of people were surprise at how well I spoke. Like my "mom" said that to me. And the neighbor lady downstairs. And just a couple of other people that I would just randomly talk to. And they were just like, "Oh.” And I thought, "Did you not think that I was educated? Or, "Did you not think I would be able to do this?" Because Anne did not express linguistic "othering," it cannot be used to analyze her transformative learning.

What is each student's racial, economic, gender, and cultural identity? How does each student's identity impact her worldview and transformations (or lack thereof) during travel? Anne was from the wealthiest suburb of a large Midwestern city. During the member-check interview, I reviewed census data about her suburb and explained that the median income and race information described an upper-middle class, mostly White demographic. Anne said that her family fit comfortably into that definition. However, because individuals occupy multiple contexts simultaneously, it was be difficult to tease apart from the data collected the role of the influence of Anne's class and racial privilege played in contrast to her abuse (Baumagartner, 2012; Taylor, 2007). Because I discussed the role abuse played on her transformative learning in the previous sections, I will focus on Anne's racial and class privilege in this section.

During the first week of classes, Anne's speech was full of linguistic indicators of extreme class privilege. When she was complaining to Kynzee about her host mom making her late because she wanted to make Anne a sandwich, I wrote in my field notes that Anne talked about her host family "almost like I would about a hotel staff rather than someone I was trying to immerse with.” Additionally, her favorite thing about her host 
family in the entrance interview was that her host "mom" did not "really expect me to do anything. She goes in and she cleans my room, and she makes me food whenever I want it."

During the bus ride home from Alhambra, I overheard a discussion where the participants were describing the "good" and "bad" areas of a major midwestern city near which many of the students lived. While I observed they talked with differing levels of socio-cultural awareness, I wrote in my field notes that Anne's descriptions were full of discriminatory speech about "drug users," "murderers," "no one should ever go to that part of town," "get raped/assaulted," and, "unemployed." As I listened, I perceived that Tess (discussed later) was listening uncomfortably. Tess told me that Anne's and Amy's (not studied) speech about the areas full of "bad" stuff was her neighborhood. I will talk in more detail about this event in Tess's findings later in this chapter.

Later in the study-abroad program, Anne explained that she had a toxic work environment during her time at PSU. Even though she was still learning to communicate in Spanish, she was responsible for all Spanish-speaking clients at a bank. One time, when she finished helping a client in Spanish, her supervisor said, "I think Spanish is such a dirty language.” This really hurt Anne, so she lashed out, “At least I'm not the one married to a towel-head," referring to her boss's husband who was of Middle-Eastern lineage. When I asked Anne how she felt about her reaction, Anne said that she knew the response wasn't "PC" (politically correct), but felt that her boss "deserved" it for all of the hurtful comments about Spanish. 
Furthermore, as I analyzed Anne's data, I observed that she used the term "Spaniard" much more than any other participant. Other participants were much more likely to say, "Spanish culture," "people from Spain," or "Levantinos" (individuals from Levante). While it is correct to call someone from Spain a Spaniard, Anne's overuse of the term felt like she thought of all people from Spain as an essentialized "other." In the literature review Morgan (2010) warned, "Overemphasizing cultural Otherness ... runs the risk of exoticizing, romanticizing, essentializing, and superficializing the lived experience of people encountered through travel which is more likely to reify than transform existing frames of mind" (p. 264).

In summary, I postulate that while the study-abroad program was personally transformative for Anne, she never stated a desire toward social action in the data collected for this study, and therefore, did not experience critical-social transformation (Brookfield 2012). While Anne's time for reflection of personal values and worldview align with Morgan's (2010) assertion that reflection can lead to transformation during study abroad, she did not develop the critical social TLT outcomes Pritchard, Morgan, and Ateljevic (2011) maintain are necessary to move travel for transformation away from a vehicle solely used for knowledge production. Her transformation was a "pedagogy of the privileged," rather than a vehicle for socio-cultural transformation (Curry-Stevens, 2011).

\section{Participant Four-"Becky"-From "Sheltered Life" to Radical Inclusion}

Becky was twenty-one years old at the time of the study-abroad program. She was between her junior and senior years of college with a major in elementary education 
and a minor in Spanish. Becky was genial, yet measured in speech and action. Previous to the study-abroad program, Becky had traveled to many parts of the United States. In addition, between high school and college, Becky attended a ten-day trip to Costa Rica, in which she stayed with a host family for five days. Her host family only spoke Spanish, which Becky described as "very difficult," yet I believe this prior knowledge prepared Becky for living with a host family better than any of the other participants.

She had an American English accent with a hint of Texas Southern. The monophthongation (turning typical dual vowel sounds - diphthongs — into single vowel sounds) of that regional dialect shared similarities to vowel sounds in Spanish and made her Spanish pronunciation sound more advanced than her other speaking or listening capacities. Consequently, Ana often invited her read in class.

Becky was one of the quietest students in class, yet one of the most gracious with me in that she was always willing to talk with me and tell me about her experiences. I think she was excited that I was a fellow educator, while most of the other participants (and Dr. Green) were Spanish majors. In fact, I noticed that she often sought me out to share experiences she was having with the language and culture of Spain. However, I have the least information about her from any of the participants because she only took Ana's Levantino Art and Literature class, in which she rarely spoke unless called on to read or answer a question. Furthermore, I failed to press "record" on her member-check interview. Luckily, she agreed I represented her really well and got to the essence of her experiences in Spain. Upon realizing her interview did not record, I wrote down everything I could remember directly after the completion of the interview. 
Becky exhibited multiple experiences with culture shock and cognitive dissonance during the study-abroad program, which led both incremental and epochal transformations. Following, I describe Becky's cultural and linguistic “otherness," which can only be understood when couched in the profound inclusion she experienced with her host family. Finally, I will analyze Becky's experiences and transformations through the lenses of her racial, class, and gender identities.

To what extent do seven students experience the elements of transformative learning during a month-long study-abroad experience in Spain? In the following section, I will explain the elements of transformative learning that Becky experienced during the study-abroad program. First, I will describe the cognitive dissonance in the form of both culture shocks (negative cognitive dissonance) and culture surprises (positive cognitive dissonance), which Becky underwent. Subsequently, I discuss two incremental and one epochal transformation that she underwent during her month in Spain.

Cognitive dissonance. From all of the data collected on Becky, the only disorienting event that evoked intense emotion was the bullfight. Becky's conservative social values also experienced some culture shock by the multiple displays of nudity, teens drinking alcohol and smoking pot in public, and the "touchy feely" nature of Spanish women. These issues seemed to contradict Becky's reserved nature, so I chose to include them in the subsequent section about cultural "otherness." In this section, however, I focus on several culture shocks such as differences in meal traditions, adjusting to the different schedule of life in Spain, and the "classy dress" of Spanish 
women only created cognitive dissonance for about one week, so I struggled whether to call them cultural shocks or cultural adjustments. Following, I describe navigating airports, the amount of American brands in Spain, and other minor examples of cultural adjustments. At the end of this section, I describe some positive cultural surprises, such as using public transportation, being able to walk most places, and the callejero nature of Spanish society.

Culture shock: Bullfight. Becky was the most nuanced of any of the participants regarding the bullfight. During the bullfight, I asked Becky how she was handling the experience. With a tear streaming down her cheek, Becky said that watching the bulls die made her "feel bad," but then choked back emotion as she said "I don't like this, but it is the culture." I wrote in my field notes that I was "really impressed that Becky stayed [for the second half of the bullfight] even though she was firmly opposed." For some reason, previous to attending, Becky thought they did not actually kill the bulls. She explained:

I really didn't know that they killed the bulls. I thought it was ... just ... a show. And we watched that in class last year, and I thought [the banderillas] were just like decoration. Like, trying to pin the donkey, but they were just like, trying to get him mad. I didn't know they were [spears].... And then watching it - I just don't understand why they'd use six bulls, and I wasn't ... a fan. Because, I feel like they're torturing it, by stabbing it and letting it bleed out. And then, when they do stab it, they torment it more, until its heart goes out, and that just. [pause.] 
I don't know, I didn't like that at all. And then when it is, when it falls over, they stab it in the head! And they cut its ears off.

I realized later that Juan explained in great detail what happens at a bullfight before the participants went, but Becky only took Ana's class. She chose not to leave with Jennifer and Kristene at intermission, because she felt that she was in Spain for an immersion experience and wanted to see both good and bad aspects of the culture.

Following the event, Becky found out afterward that many young people oppose bullfighting on moral and ethical grounds, but some of the older people still love it. Even though Becky opposed the senseless torture and killing of the bulls, she told me she was glad that she got to experience it. It is also important to note that both Becky and Kynzee's (discussed later) host "moms" really built up the bullfight. They provided them with traditional Levantino dresses, put flowers in their hair, styled their hair in an updo with loose curl bangs coming down, and put traditional make-up on their faces. About the cultural aspects of the bullfight, Becky explained in the exit interview:

I liked the music and the atmosphere, and the historical coliseum -that was cool.... My "mom," ... likes it, and so when we were talking about it...I'm like, “Ah, I know, it's cool to see it, but... I don't really like it, you know, how they torture [the bulls.] Especially six of [them.] And they do it like, five days [in a row].” And I understand we, like, slaughter cows, but I'm pretty sure just like, "boom," and done, not like, you can see their heart...

At this point Becky became too emotional to discuss it any further. After Becky regained her composure (and in an attempt to encourage her to move on from discussing 
the pain of watching the bulls die), I asked her if there was something equivalent to bullfighting in the United States. Becky replied:

It's kind of like rodeos. I was never a fan of rodeos. I've been to ... a couple, but ... they don't kill them and torture them. But they do, I feel like, kind of torture what's it called, bull riding? Yeah, I'm not a big fan of that, either. I think that's kind of cruel.

Meal traditions. One of the most difficult traditions for Becky to swallow was getting used to various meal traditions in Spain. Initially, she was shocked how her host family often ate salads and appetizers off of a shared plate (which is common in Spain). In the entrance interview she said:

Last night we went to a ... restaurant.... My host-mom would cut [the food] up, and then we'd just ... eat off the same plate and ... use the same knives.... And I'm like, "Uhhh, am I supposed to eat that? I mean, you just like licked your fork and then touched all our stuff."

Another tradition which was a difficult adjustment for Becky was having the biggest meal of the day at two o'clock in the afternoon. Furthermore, she was uncomfortable with how many courses her host family ate at this meal. In the entrance interview she said:

We always have ... salad first ... and it's huge.... I'm like, “Okay...this is ... lunch. That's good. I'm good." And then she brings out soup, ... and it's a huge portion.... And so I won't eat that much and then I feel so rude, because I'm not eating that much, and then she... [brings] appetizers. And then she ... brings out 
the main course.... And then after, she's like, “Oh, you want ice cream?” ... I was ... going to throw up if I [ate] any more.

Finally, Becky was concerned that her host family always asked if she wanted beer or wine at the main meal of the day (also common). In the entrance interview she stated, “At our meals, they're always ... asking me if I want wine, or beer. And I'm just like, 'No."”

In the member-check interview I asked Becky if the differences in meal schedule, eating habits, meal quantity, and alcohol content continued to concern her. She told me that after the first week she "got used to it" and "it was no big deal."

Cultural adjustment: Daily schedule. In addition to the timing of meals, the daily schedule also took Becky a week or so to become adjusted. She told me in the first week that she "was surprised about how late everything is." In the entrance interview, Becky contrasted her life in the United States with her host family in Spain. She said:

I usually get up at like 6:00 a.m., and get ready, go to school, go to work, and then I'm in bed by like 10:30 p.m. And [in Spain], my host "mom" would go out at like 10:00 p.m., stay out, come and just leisurely do stuff, and then she's like, "Yeah I usually normally watch TV until about 2:00 or 3:00 a.m., and then go to bed." I'm like, "What?! 2:00 or 3:00 a.m.?!"

Cultural adjustment: "Classy" dress of Spanish women. In the entrance interview, Becky explained that it was "common for girls... to dress like sporty" in her college town, while in Spain "everybody dresses so classy." The main reason that this 
caused any concern at all for Becky was, she simply did not own or bring along "that many very classy clothes." She continued:

I was shocked... My host mom, always [dresses] in khakis, or, looks very nice. And my host dad always has like a collared shirt on, or, not jeans or anything. Like, designer bags. I don't know if that's common everywhere, but she has like three designer bags.

In the member-check interview, when I asked Becky if she experienced any reverse culture shock, she said that she became so accustomed to the "classy" dress in Spain that it took her a couple of weeks back home in the United States to see everyone walking around in "jeans and sweatshirts." Furthermore she maintained that for a few weeks, still got dressed up to leave the house.

Cultural adjustment: Religion and politics. Becky was fascinated that her host family regularly discussed both religion and politics with her. In the exit interview, she said, "You know how people say don't talk about your religion or politics? Yeah, we talked about both." She was surprised to learn from her host "dad" that most Catholics in Spain do not habitually go to church. Moreover, Becky was also interested by her host "mom's" criticisms of the Catholic church. Becky said:

My “mom” was saying how she doesn't like ...the Catholic religion here, because all the churches are huge.... And she was saying how she doesn't like them because they... just take your money and don't help the poor. And so... I was like, "Yeah, I mean, that's kind of like the United States. A lot of people don't 
like ... those huge, huge churches 'cause they should be helping the poor... It was kind of awkward, but it was interesting, to see their view."

Similar to discussions about religion, Becky's host family often discussed politics in Spain with her:

When we learned about [the dictator and the Spanish Civil War at PSU], it was ... the negative things about Franco. And that's what we learned. And so, my hostdad was saying that when Franco started, a lot of people didn't like him. But, towards the end of his dictatorship, a lot of people liked him because, I guess, there weren't as many poor people and everybody had a job or something. But, maybe that's because women didn't work. And so, now, in the culture, like, women work, and that might be why the unemployment rate is so...high? I don't really know. But it's funny, though, because [my "mom"] said she likes Franco, and liked the dictatorship, but she wants like a democ... well, not a democracy, she is for the republic. And so I'm like, that's kind of interesting, you know? Kind of wanting, liking Franco and the dictatorship, but wanting a republic. She does not like the king and queen. She thinks they just take money and don't help the poor. Which, I think, I can kinda see that, too, because... when the king was coming into office, that parade and everything was so elaborate, and 7,000 security ... checking everywhere for bombs, and she's like, "This is crazy ... there's (sic) kids on the street not eating, and here they are having ... days and days of ... elegance." Which I understand that, where she's coming from. 
In the member-check interview, Becky explained that although her "politics did not change," she seemed to authentically enjoy the chance to understand Spain in a more complex way than her Spanish history classes previously taught her. Becky did not take Juan's class on the history and culture of Spain because she had already taken a similar class at PSU. However, I believe she would have enjoyed the more nuanced, politically charged style of Juan's teaching.

Becky's was controlled, thoughtful, and reserved in both speech and action. Previous to the study-abroad program, her version of propriety was that uncomfortable topics should be avoided. Nevertheless, although Becky was uncomfortable discussing religion and politics, she valued the relationship with her host family. Therefore, she listened and respected what they had to say. This respect and permeability align with both Morgan's (2010) and Ross's (2010) finings that valuing the host culture is necessary in order to move TLT in a critical social direction. Moreover, Mezirow (1998) maintains that avoiding sensitive discussions inhibits critically reflective thought. Consequently, because Becky moved past her discomfort, she learned knew ideas and new ways of thinking about politics and religion, reframing how policies impact the daily lives of normal people like her host family, as well as her future students.

In addition to religion and politics, there were myriad other cultural adjustments Becky experienced. However, in the member check interview when I asked her about them, she stated that none of the following were a "big deal," and the way she talked about them made me feel like she was noticing differences more than experiencing cognitive dissonance. She was disappointed to see how many American brands, clothing, 
and stores there were in Spain. Likewise, it frustrated her to see how many people spoke English. Furthermore, coming from PSU, which is a regional university, Becky found it fascinating to see how large LU was. Lastly, like some other participants, she experienced confusion in airports, but not to the degree of the other students previously mentioned.

Cultural surprise: Callejero-nature of Spanish people. When I asked Becky in the member-check interview what she missed most about Spain, she explained that she missed "public transportation, walking everywhere, and going out with friends more." Becky described herself as "sporty," and explained that she liked to eat healthfully and exercise regularly. In the entrance interview I asked her what she enjoyed about her first week and she explained, "Public transportation, and how big it is here. And, being able to walk everywhere.... I love that. I wish I could do that in the United States, 'cause I love walking everywhere. It's free, it's good for you.”

It seemed that Becky tied public transportation, walking, and socializing together. As Becky was explaining this concept, I realized there is a word in Spanish that most people in Spain consider a characteristic of Spaniards. They call it their callejero nature, meaning that most people would rather be outside socializing than sitting in their apartments. Becky said in the entrance interview, "The streets are packed with people, and not just young ... it was everybody: older, younger, families." In the member-check interview, Becky told me she became accustomed to, and even came to appreciate, both the later start times and callejero nature of the Spanish people. 
Transformations. Below I describe two intertwining incremental transformations, followed by one epochal transformation that occurred during Becky's study-abroad program in Spain:

Incremental transformation: Live like the Spanish. Becky appeared to genuinely enjoy the lifestyle of Spain. In her exit interview she explained:

When I [go] back [to the United States], I want to try and live more like the Spaniards. I thought they were just kind of like, relaxed. 'Cause I feel like in the United States, a lot of people are so caught up on being rich, and like making a lot of money, and I feel ... here, they're just like, "Oh yeah, we'll work ... so we can go out to eat with my friends." You know, just enough to ... have fun. In the United States I feel like we just work, work, work, work, so we can buy a house, and buy a big boat, and buy another house, and just. I feel like our morals ... are ... kind of selfish and kind of greedy. And I don't feel like they're like that here as much.

Incremental transformation: Pride in teaching profession. Linked to Becky's belief that American's are too focused on money, came an increased appreciation for the role that teachers play in society from her discussion with her host family and others in Spain. Additionally, it seemed that as time went on her pride in being an education major increased. I perceived that the Spanish opinion of teachers seemed to have a direct influence on Becky's augmented pride in her future profession. In the exit interview she stated: 
In the United States, I feel like people look down on teachers, because they don't make as much money.... At work, when people [say,] "Oh, you're a student..." I'm like, "Yeah, I am.” And they're like, “What's your major?” I'm like, “Oh, I'm gonna be a teacher of Spanish." And then they'll be like, "Why do you wanna do that?" I'm like, "'Cause it's the best job!” I was like, “'Cause I love it!” And I'm thinking about doing special education, or ESOL, or both, or getting my certificate in that. And they're like, "Why would you want to do that? You have to work for that." And I'm just so offended. And then, [in Spain,] when people ask me what I want to do, I'm like, 'Yeah, I want to be a teacher.' And they're like, "Oh my gosh, that is so amazing! That is so cool!" ... It's just so different when I tell. I feel almost ashamed sometimes when people are like, "What do you want to do?" I'm like, “A teacher." And they're like...they'll be like, "But they don't make a lot of money..." I'm like, "So?"

In the member-check interview, Becky reiterated that although she wanted to be a teacher before the study-abroad program, her discussions with Spaniards made her more proud of the profession that she has chosen.

Epochal transformation: Education as social action. In the exit interview I asked Becky what changes she saw in herself from spending a month in Spain. She told me, "I feel like this trip has made me more sympathetic to ... foreigners in our country." Furthermore, throughout the interview she discussed a desire to become certified in both English for speakers of other languages (ESOL) and special needs education. Although Becky did not recount any severe marginalization, she maintained that the linguistic 
"otherness" she experienced prompted her to act to make society better for English learners within education. She said, "I think it makes you more sensitive because you have felt the culture shock." She also said:

I think [study abroad] makes you more sympathetic to foreigners... [and] how they feel coming here.... I work [in a] town [that] has a lot of Hispanics. And so, they come into the store I work at, and now I feel like I'll be more sympathetic towards them, because I've had to do that: go into stores and ... try and explain what I need and want. But, it also helps ... because I know Spanish, so I can ... know what they're talking about.

Additionally, Becky further explained her conclusion to get an ESOL endorsement while participating in the study-abroad program in the exit interview, "I work in a school, and ... our town has a [large] Hispanic population. And I feel like a lot of teachers aren't very sympathetic." She also maintained that the study-abroad program would "help a lot in my teacher career, just being able to relate better to the students." Moreover, Becky explained during the member-check interview that when she volunteered in classrooms in the United States, she felt that many educators wanted to force English learners to assimilate to solely English and to adapt to "American values." She said:

A lot of ESOL teachers... don't know ... another language, they don't have culture, they've never studied abroad.... But here they are, working with ... other cultures, and trying... to like change [English learners], or make them learn 
English instead of trying to incorporate both. And so, I [will] try to incorporate both cultures and try to keep their culture, too.

Throughout the study-abroad program, Becky explained how on this trip she came to feel the importance in celebrating and knowing both cultures for English learners. Therefore, she hoped she could teach her native Spanish speakers to know their own language, heritage, and culture as well as the United States language and culture.

Furthermore, Becky maintained that this multicultural approach should benefit her native English speakers as much as her English learners. Although Becky did not participate in the study-abroad program as a service opportunity like Coghlan and Gooch (2007) recommended, a desire to provide service to her educational community resulted from her time in Spain. I posit that the deep value Becky placed on the relationship with her host family led her to value the host culture, as well (Morgan, 2010; Ross, 2010). Consequently, from Becky's words in interviews, it appears that a desire to impact the lives of "others" upon her return to the United States grew from this relationship.

What role did encounters with cultural and linguistic "otherness" play in the transformative learning (or lack thereof) of each participant? It is impossible to understand any cultural or linguistic "otherness" that Becky experienced in a contextual vacuum void of the extreme inclusion she felt from her host family. Therefore, for the following section, I will begin by explaining Becky's sense of cultural inclusion. Following, I will describe cultural "otherness" she underwent. Lastly, I finish this section describing Becky’s linguistic "otherness." 
Radical cultural inclusion. Becky and Kynzee (discussed later) were assigned host families in which the two host "moms" were best friends. The housing coordinator informed me that both families enjoyed housing study-abroad students and took great pride in showing off the culture of their hometown, Levante. Of all the times of the year to study in Levante, the festivals that happened at the end of the study-abroad experience were the most important experience and celebration of Levantino culture. Both sets of host parents were empty nesters in their 60s. From the clothes they wore and the part of town in which Becky's family lived (and from the explanation of the housing coordinator), it was evident that they hosted students because they enjoyed it rather than out of financial necessity. All culture shocks and surprises that Becky underwent must be understood in the context of the respectful immersive experience that Becky enjoyed with her host family. Indeed, it seemed that Becky shared observations out of respect for a fellow educator, not out of deep emotional disorientation or cognitive dissonance. Her experience was unlike any of the participants discussed up to this point.

After only a week in her host family's flat, Becky informed me that it already "like home a little more now." She also told me she was very comfortable with her host "mom" because "my host mom is kinda like my mom back home. She's very ... relaxed, and compassionate, [and] patient." Furthermore, In the exit interview, Becky said, "I feel pretty immersed in the culture, 'cause my host-parents are always taking me somewhere—restaurants, little bars, little ice cream places."

While Dr. Green and I were walking around during the festivals, Becky's host "mom" stopped Dr. Green to inform him that she was having "more fun" with Becky 
than she had ever had with a study-abroad student in her years of hosting. Regarding the festivals, Becky told me that her and Kynzee's "moms," "drug us out to everything." Their host "moms" dressed them in traditional costumes and tried to get them to go to "every festival." Becky said that the "party" lasted only during the five official festival days, but throughout the whole program. She maintained that they played "Uno until like 2:00 in the morning" every night. Her only complaint about the host family situation was, "I do think it would be cool, though, to meet, like Spaniards our age, too. Just to learn their culture from their perspective, you know? 'Cause my host-parents are pretty old. I mean, they're fun, though.” In the member-check interview, I asked Becky what she would change about the host family situation. Becky said she loved the experience and loved that her host mom "wore her out" and kept "pushing her to experience more." Becky maintained if she could do the study-abroad all over again, she would not change anything about her living situation.

Cultural "otherness." From all data collected, Becky appeared to have an overwhelmingly positive experience compared to the three participants described earlier. Like I stated above, Becky's "otherness" can only be understood through the lens of the radical inclusion she felt from her host family. Additionally, I must also restate that I do not think she would have ever looked upon the experiences described in this section as marginalization.

Nevertheless, from my observations Becky experienced an "otherness" that was a break from her own family's cultural norms. Becky was very socially conservative. By conservative, I do not mean that she was necessarily politically or religiously 
conservative. Rather, Becky was very rational, measured, and emotionally controlled. However, in no way should this description of her personality taken as cold. Quite the opposite, Becky was warm and affable. Yet there seemed to emanate from her a sense that cultural propriety meant not discussing or mentioning sensitive topics like religion or politics. When trying to describe her sense of propriety, Becky stated, "Maybe I lead a sheltered life."

The first experience that was significantly "other" for Becky's cultural norms was the "touchy-feely" nature of Becky's host mother. Becky was surprised at how quickly her host "mom" started giving her besitos and calling Becky "mi amor" (my love). While this was something that Becky became used to, and even came to appreciate, she said it was a significant departure from her family growing up. However, in the member-check interview, she said that whenever she saw Tess and Kynzee (both discussed later) on PSU's campus, they still greeted each other with besitos and caring terms like "mi amor." Another incident that Becky found extremely different from her self-proclaimed "sheltered life" was seeing teenagers smoking, drinking, smoking marijuana, and "peeing in the streets" during the festivals. She repeatedly used the term "not legal stuff" when describing her experiences with the festivals. When I asked her if by "not legal stuff," she meant marijuana, Becky said, "Probably drugs.... They were smoking stuff that ... didn't look like cigarettes; I don't know if that's marijuana."

Finally, and most-importantly to Becky's sense of propriety, she seemed to find nudity disorienting. She used "shocking" like other participants to describe it. However, it more closely matched cultural "otherness" for the amount of times it came up in her 
interviews. In the entrance interview she stated, "It was really shocking when we went to the beach and, like almost everybody, a lot of the women, were topless, so they had their children topless."

One experience demonstrates an experience so far removed from Becky's sense of propriety that it caused me to describe these experiences in the section of cultural "otherness" rather than in the culture shock section above. One day in class at the beginning of the third week, Ana was lecturing on the artist Joaquín Sorolla. She showed the painting El Bote Blanco, in which two pre-teen boys are swimming by a rowboat in the sea. Through the reflective water of this impressionistic painting, it is possible to make out the naked buttocks of one of the boys. Ana asked Becky if she would put this painting in her house. Becky responded, "Of course not, the people are naked." Ana then explained that the naked human body is seen differently in Europe than it is in the United States. Ana countered, "Can I show you a painting I have in my house?" Next, she showed (on the 100+ inch screen) the painting L'Origine du monde by French artist Gustave Courbet in which the vulva, abdomen, and breast of a female subject are prominently displayed. Several of the female participants gasped. I wrote in my field notes that "Becky, Kynzee [discussed later], Jennifer, and Kristene looked very disturbed." Becky turned red and was silent. I tried several times to get her to open up about the experience, but she would never discuss it because it seemed to be too extreme to for her to discuss. I think discussing the painting with a male was even more of a break from her stated sense of propriety than merely discussing the painting (I will address the limitation of my maleness in the Discussion chapter). However, in the 
member-check interview, Becky said that she eventually "got used to" seeing women and children without bikini tops.

Lingustic “otherness." Becky often came between classes with funny stories about her misadventures in Spanish. About halfway through the study-abroad program recounted a story in which she was trying to command the dog of her host family to go up the stairs. No matter how many times she commanded the dog to sube (go up), it just stared at her. Moments later, the neighbor approached and yelled, "isube!" to the dog and it immediately ran up to her host family's apartment.

About the same time, Becky and Kynzee told me in between classes that their linguistic incapacity to make plans in the future tense in Spanish was negatively impacting their relationships with their host "moms." I wrote in my field notes: [Becky and Kynzee] said that in their relationships with their host moms, they think they are making plans, but then their moms aren't there, or their moms make plans and then they aren't there. [Becky and Kynzee] said in the university they talk a lot about literature, but that is always either in the present or the past tenses. They said that they are picking it up, but they worry about messing up because they do not want to offend or not show up for plans with their host families. I am exited to see that they are worrying about their relationships with their host families.

Because both Becky and Kynzee deeply valued their relationships with their host families, they asked me for a quick lesson of different ways to speak in the future tense in Spanish. 
Ironically, Becky's capacity to speak in the future tense created an uncomfortable situation her last night in Spain. When Becky's host family found out that she needed to stay one day longer than her contract for the study-abroad program, they invited her to stay an extra day at their house. Although Becky had planned to go to a hostel, she decided to stay because they had all formed such a positive relationship. As Becky was about to leave the door, her host "mom" asked for money. Becky had no cash because she spent her last few days trying to get rid of all her Euros. When she went down to get some money from the ATM, the ATM ate her card. She gave her host family forty dollars, but then did not have money to eat on her plane ride home. However, Becky was much more concerned about her relationship than the money or the inconvenience, even though a night in a hostel would have cost her only twelve Euros.

Although Becky did not speak the best Spanish of the participants, it appeared as if she experienced the greatest immersion in the language, due to the extensive time spent with her host family, who did not speak English. Moreover, Becky said in the membercheck interview, that besides noticing Americans do not dress as "classy" as the Spanish, her only major adjustment returning home was speaking English again. This culture shock of returning to English indicates a greater degree of immersion in Spanish. I postulate that it was Becky's immersion, more than her sense of linguistic "otherness," that impacted her development of cultural awareness. This hypothesis aligns with Goulah (2007) found that students who learned another language while immersed in that culture developed critical socio-cultural attitudes about their home culture. 
What is each student's racial, economic, gender, and cultural identity? How does each student's identity impact their worldview and their transformations (or lack thereof) during travel? In this section, I will describe how Becky's class and permeability may have been related. Following, I explain her experience overcoming her desire to avoid sensitive topics, which I relate to the CRT concept of colorblindness.

Class and permeability. Becky's parents were both working professionals; her dad was a director for an apartment company and her mom was a school psychologist. Becky felt that she grew up "solidly in the middle class," but her parents' divorce during her senior year of high school financially stretched the family. She explained that paying for college probably would not have been "an issue" before her parents' divorce, but that "both parents try to place the financial burden on the other one." This caused Becky to have to pay for almost all of her college expenses, including the study-abroad program. Part of the reason Becky only took one of the LU classes during the study-abroad program was due to her financial limitations. Consequently, Becky felt that calling herself "middle class" was fair.

Acquiring enough money to pay for the study-abroad program was a difficult task. Because Becky mentioned this difficulty several times (phrases like "I finally had the money to do it this year."), I asked her if she minded me asking how she paid for the trip. Becky explained that she paid for most of the study-abroad program herself through scholarships, asking for money for Christmas (in lieu of gifts), and by working three parttime jobs while going to school. Additionally, her grandparents knew how important this 
trip was to her and gave her about one-quarter of the expenses, fearing she would not be able to go otherwise.

During high school, Becky made a goal to study-abroad in college. Her sense of purpose in travel (Morgan, 2010) can be seen in the following excerpt from her exit interview, "When I was a senior in high school, visiting schools, I knew I wanted to go on a study-abroad trip to Spain. That was ... my ultimate goal." Becky believed that this trip was a singular opportunity that could never be repeated. She expected the studyabroad experience to be transformative, and it was.

More than any of the other participants, Becky developed a permeable, rational, and reflective worldview that TLT scholars discuss as a prerequisite to transformative learning (Ettling, 2006; Mezirow, 2000, 2004, 2012; Taylor \& Cranton, 2012; O'Sullivan, 1999). As will be described later in the findings of Tim and Kynzee, I came to question the word "willingness" in Mezirow's (1998) writings, and now prefer the word "permeable" that he often uses interchangeably. For example, Tim (discussed later) had a typical response of, "Hell yeah!" to optional activity offered the participants. There was no participant more "willing" than Tim. However, the study-abroad experience was not particularly transformative for him. In contrast, Becky's permeability seemed to have inspired critical reflection of her beliefs about good teaching and led her toward a desire to be an agent for social change within her classroom and educational community (Baumgartner, 2012; Merriam \& Kim, 2012). Likewise, Becky's epochal transformation of educator for linguistic and cultural justice aligned with Pritchard, Morgan, and 
Ateljevic (2011) view that opportunities for intimate discussions with the host culture uniquely positions travel as a vehicle for critical social TLT.

It is extremely important to further note that Becky's rejection of the discriminatory assimilation-based teaching she had observed in the United States status quo did not converge with her personal interests. Rather, her desire to get new teaching certificates in ESOL and special education required more work, education, and money. Therefore, Becky is the first participant studied thus far to experience both Mezirow's (2000) version of transformative learning, as well as critical social transformative learning (Baumgartner, 2012). To be considered critical social TLT, the transformations must move beyond merely empowering participants to change their lives for the better and requires that participants act to improve society, as well (Merriam \& Kim, 2012).

As was explained in the introduction to this chapter, I placed the participants in an order of decreasing socio-economic privilege. The previous three participants, Kristene, Jennifer, and Anne all self-identified as upper-middle class. Becky is the first participant to identify as middle, rather than upper-middle, class. Furthermore, she was the first participant to earn most of the expenses for the study-abroad program herself. As I analyzed the data, I questioned if Becky's difference in class privilege may have impacted her permeability, and thus her desire for socio-cultural action compared to the other students. Because of the small sample size $(N=8)$ and qualitative nature of this study, it is impossible to prove causality or to generalize findings (Marshall \& Rossman, 2011). Nevertheless, for Becky, the hard work she put in to earning money for the studyabroad program led her to experience a deep sense of purpose (Morgan, 2010) and a 
permeability toward the host culture (Ross, 2010) not seen in other participants discussed thus far. Consequently, for Becky, her difficulty in acquiring funds directly impacted her desire to respect and value the cultures of her future students.

Racial privilege and the myth of colorblindness. When discussing the reasons for wanting to seek an ESOL certification, Becky stated that she wanted to advocate and teach English learners in the United States because the study-abroad program made her "more sensitive because [she had] felt the culture shock." This statement aligns with Johsnon-Bailey and Alfred's (2006) position that marginalization may be the most powerful transformer. However, after extensive review of Becky's data, I did not find that Becky experienced "marginalization" as much as she experienced cultural immersion and adjustments.

Becky's liberation from avoiding sensitive topics and immersion in the host culture played a much greater role in her critical social transformation. Even though it is human nature to protect oneself from topics that are delicate, sensitive, or contrary to an individual's frame of reference (Mezirow, 1998), Becky seemed to respect her host family's (and others') insights enough to break free of her personal culture norms of not discussing sensitive topics like politics and religion. Moreover, Becky's critical social transformation parallels Coghlan and Gooch's (2011) position that by stepping away from an individual's socio-cultural context, she can develop critical reflection, and therefore, increase her capacity to understand and act in the larger political, economic, and socio-cultural domain. 
The closest idea I could find to Becky's previous refusal to engage in political or religious discussion in the literature was Leonardo's (2009) critique of colorblind discourses that reinforce institutional racial privilege. Becky's desire previous to the study-abroad program to avoid sensitive subjects reinforced her own racial privilege through the "psychological and material benefits from an unearned position in society" (Leonardo, 2009, p. 273). Furthermore, because the unearned benefits of being socially constructed as White have been obfuscated by unjust laws and social norms in the United States, it is extremely difficult for a person of privilege to become aware of these unearned benefits (Brah \& Phoenix, 2009; Brookfield, 2012; Gillborn, 2009).

Nevertheless, Becky came to the decision to reject her racial privilege and work for racially, linguistically, and ability marginalized students. In essence, Becky came to understand that social action within her future profession was a necessary outgrowth of her time in Spain (Ladson-Billings \& Donner, 2005).

\section{Participant Five-Tim—“Willing," but without Observable Transformation}

Tim was singular in several ways. First, Tim was the only male participant in the study-abroad program. I asked him a couple of weeks in if it was acceptable to write about him as a male because if any of the other participants ever read my dissertation, they would be able to identify him. He said, "You can put my full name and phone number in your dissertation. I am who I am." That leads to the second characteristic. Tim was willing to discuss anything. Initially, I believed my maleness allowed me to talk with Tim openly, but as time went on and I got to know Tim better, I realized he was completely open to discussing any topic any time. In the LU classes and cultural 
excursions, topics like politics, money, and sexuality (that all seemed taboo for most of the female participants) were welcome and exciting issues for Tim to discuss his views. In contrast to Jennifer, who was a political science major, Tim's views on politics seemed completely self-taught. In the final section of Tim's findings, I will discuss my observations that this openness might be seen as a form of Tim's, rather than my, male privilege. Third, Tim was the oldest participant at twenty-eight years old (the next oldest participant was twenty-two years old). Lastly, Tim was the only participant in this study with zero observable or stated personal or socio-cultural transformative learning. While no causal links can be determined as to how Tim's age and gender played into his apparent lack of transformation due to a single-participant sample size (Marshall \& Rossman, 2011), Tim's experiences in the study-abroad program serve as an interesting case study to analyze the transformative learning of the other participants. Furthermore, as this research utilizes constructivist grounded theory for data analysis, the purpose is not to form causality (Charmaz, 2003). Rather, the purpose is to promote the narratives and experiences of the participants, who served as co-collaborators in data collection and analysis in participative storying (Pritchard, Morgan, \& Ateljevic, 2011; see also Vogt, Gardner, \& Haeffele, 2012).

Similar to the findings of previous participants, I will discuss Tim's experiences through the lens of the three research questions of this study.

To what extent do seven students experience the elements of transformative learning during a month-long study-abroad experience in Spain? First, I will discuss 
cultural surprises Tim experienced followed by an explanation of Tim's lack of a transformative learning experience in Spain.

Cultural surprises. Tim never mentioned culture shock that would count as cognitive dissonance at any point throughout the study-abroad program. Indeed, in the exit interview he stated, "I would say my culture shock did not last very long." However, when I asked him about what some of the culture shocks he experienced were, he stated that he could not think of any. This was true for all of the formal and informal interviews I conducted with Tim. Nevertheless, Tim did experience what the participants labeled "cultural surprises" throughout the study-abroad program, which will be discussed below.

Family. As mentioned with previous participants, the king of Spain abdicated the throne to his son during the first week of the study-abroad program. Tim enjoyed watching a heated debate one night among members of his host family about whether the monarchy should continue or if Spain should form a republic. In the entrance interview, Tim stated:

Yesterday they were watching the news, about the abdication and everything, and they got into a super heated ... argument. All three were talking full-blast ... and I understood almost none of it, but ... the whole thing just tickled me pink, because it was such a 'family' thing to have happen.... Just this heated debate about politics, and they all had their own opinions. It was family.... It was beautiful. I loved it. [My host "mom"] kept ... crying to me, "Oh, sorry, I'm talking too fast. Sorry, you don't understand it. Sorry we're starting to yell a little bit." I was like, "Oh no, I think this is great. Keep going...." I loved it. 
Walking. One day Ana asked the students what they were enjoying most about Spain after a week of living there. Several of the students commented that they enjoyed the opportunity to walk much more than they did in the United Stated. Tim said (with disgust in his voice), "Some people drive their cars to the supermarket when it is only a block away!" When I followed up in the member-check interview about this joy for walking, Tim reflected:

I've realized that what made walking so fun was that there was so much to look at. Like, old buildings, old parks, old trees, or even new buildings that have been designed really cool. You know, there's a lot of visually stimulating things on a walk in the city, whereas here in [the United States] it's so homogenous that it's just not really visually stimulating.

Bullfight. During the bullfight, I wrote in my field notes, "Tim seems to be having the time of his life." He was actively cheering for the matador, drinking wine from a bota (a leather bag for holding wine that is traditional for bullfights and soccer games), and mimicking the excited screams of the crowd. Tim explained that although it was hard to watch, he felt it showed a "respect for the life of the bull" and what it "means to eat meat." In the exit interview, however, he explained his emotions in the bullfight with more nuance than I observed during the event:

I really enjoyed the cultural experience of it. It was hard to watch. I don't think I need to watch another one.... One thing that interested me is by the sixth bull, I was...acclimated to it.... The sixth bull didn't bother me as much as the first bull did.... And I ... was far enough removed from my feelings of the first bull that I 
noticed it as being, like, “Oh, I adjusted to this pretty quick.” But I don't want to see another one. It was fun, though. I did enjoy it. Well ... I enjoyed the experiences of it. I wouldn't classify my emotions as being, “Oh, this is awesome!" It was not a sporting event or any sort of similar thing.

Furthermore, when I asked Tim if there was an American equivalent to the bullfight, he said:

I don't know, because... the bullfights, as brutal as they were... it was possible to see that ... bulls carry such a cultural importance to Spain, that, even though it was brutal, it was ... out of respect for the animal that they have these events. And I can't even think of an American comparison, 'cause I'm not sure we possess the necessary respect for anything. I would say our equivalent would have been our...annihilation of the Native American tribes.... Well, not that wasn't even out of respect, though, that was just 'cause we wanted their shit... I don't think we have a parallel.

In the member-check interview, he stated that alcohol may have had some role in his outward appearance of enjoying the event, despite the brutality of it:

Definitely the more wine I drank, the more I enjoyed it.... I did really enjoy the experience. But, it was hard to watch, man. I definitely have no desire to see a second one.... I loved the opportunity to experience that part of Spanish culture, really.

Tim also explained that he was fascinated at the flood of emotions that arose during the bullfight. He compared it to going to a sporting event wherein he became "caught up 
with the crowd in terms of, 'Look at what we're watching! Yeah!'” He explained that he believed that emotions have a "tangible quality" that affected him. He finished with, "Any sort of arena event or stadium where you have a lot of people, and just a lot of emotion, it's a fun thing to be a part of."

The Cathedral of Granada. Tim was observably overwhelmed by emotions when he toured the cathedral in Granada. Immediately afterward, I asked him if he would explain what the experience was like for him. I recorded this informal interview. Tim replied:

The cathedral in Granada blew me away.... It's huge.... In my pictures there's (sic) these little people walking around, and then this giant cathedral.... People that size built that cathedral. They were unified and driven by faith and love, and, probably slavery, to build this just immensely beautiful structure.... While we were there, every time I started to think about that ... I could feel myself like starting to... I didn't tear up or cry, but if I thought about it for too long, I would have.... I could feel that happening. It was just like, “Oh man! This is just so impressive!"

In the member-check interview, Tim stated that he still was overwhelmed thinking about the enormity and beauty of that edifice. He said, "Still thinking about it ... a well of emotions [builds] in my core," and then reiterated the feelings described above.

"Radical" politics. Tim enjoyed discussing and debating different political systems in both Ana's and Juan's classes. Furthermore, he enjoyed the more complicated system of parties in Spain rather than having essentially two competing sides that are 
prevalent in the political discourses in United States media. Tim felt that the Spanish openness to political discussion was grounded in the inability to openly discuss politics during Franco's dictatorship from the 1930s through the 1970s. In the exit interview Tim maintained, "After such a long period ... of ... having their opinions repressed ... I'm not surprised at all about how politically-minded people are." He also compared Spanish and American politics in the exit interview:

I think it's so second-nature to us, that we don't really give [political opinions] the amount of respect or voicing that [they] really ... deserve.... [In Spain,] I think it's awesome that people want to be a part of their politics, whereas all we want to do in the United States is... argue about it, and our side to win. We don't really care if their policies are good or bad, we just want our side to win. We're just so damn competitive; it really bothers me.

Throughout the classes, Tim was a defender of more radical political systems like communism and anarchism. As discussed in Jennifer's findings, Ana called upon Jennifer to explain what anarchy was. Jennifer explained in the United States anarchy or communism were considered "bad" political systems. Tim and Kynzee (discussed later) interrupted with, "Not necessarily." Tim explained socialism and communism are from the same roots, but communism is more "extreme." Ana further commented that communism might have a system that is more "radical" but that in Spain it was not acceptable to call any system "malo." Rather, she implored the students to discuss the ideas behind each political system. Tim nodded in agreement.

Throughout the study-abroad program, Tim asked and answered questions about 
politics, and became especially interested in systems that were different than the United States. For example, as Juan was lecturing on the balkanization of many countries throughout Europe, Tim asked, “What happens if Catalonia leaves Spain?” Juan followed his question with a long discussion of the economic, political, and European Union ramifications if Catalonia were to leave Spain.

Tim also repeatedly voiced his fascination with the constitutional monarchy of Spain. He felt that the king was a "lightning rod" that citizens could "hate," while the president could actually "get stuff done." In the exit interview he explained his opinion of the constitutional monarchy:

I do like that they have a king.... He is a lightening rod.... People have superdivisive opinions about the royal family, but I haven't heard anyone say anything about their president.... It's interesting to me that people choose to focus on [the royal family] so much, and not the actual governing body. And, I am a little jealous of that, because our presidents can't accomplish much, because they're hamstrung by public opinion, and divisive politics.

Tim further stated in the member-check interview:

I thought a constitutional monarchy was a pretty awesome system. Because it really did allow the government to actually function as a government.... But if they've got a lightning rod to hate against, then everyone else is fine.... [Spanish citizens say,] “The royal family's screwing everything up! They're going on hunting trips when the world's all crappy!" Meanwhile, the government is actually like, "Oh, everyone's really mad about this, we should, you know, 
respond to [this issue]."

Nevertheless, Tim told me that he was glad that he lived in the United States and was not advocating a shift to a monarchy. In the exit interview, I asked Tim if anything in this trip changed or more firmly embedded him in thoughts about politics. He maintained that nothing had changed.

Festivals. Like the other participants, Tim enjoyed the festivals. In the exit interview, he said, "The fireworks have been awesome.... It's (sic) gonna make the Fourth of July show a little disappointing. Additionally, Tim told me that the festivals were "damn impressive" and that he was "bummed" when the festivals were over.

Lack of observable transformation. What set Tim apart from the other participants (aside from being male and older) was an apparent absence of transformation. Repeatedly, Tim used the phrase "smooth sailing" to describe his experience in the study-abroad program. I asked Tim if he noticed any changes in himself or in the way he thought during the exit interview. He replied, "Nothing specific or super-clear comes to mind.... I'm positive it's affected me. I don't know how it's affected me and I'm sure those changes will unfold over time." I followed up in the member-check, alluding to and re-asking the question just mentioned. Tim said, "No.... I would say ... I fell back into my old patterns pretty quick once I returned.” Like I have stated with previous participants, it is possible that the study-abroad program impacted Tim's worldview and that he simply could not articulate it, or that he will not realize the transformational nature of the experience until after the data collection period ended. 
However, during the study, I did not observe and Tim did not express any transformations of his frame of reference.

“Willingness.” Reviewing Tim's data has led me to question Mezirow's repeated use of the term "willingness" in his writings. Mezirow (2000) indicated that willingness to listen and speak was a key component in TLT (see also Mezirow, 1996). Furthermore, Brookfield (2012) maintained that participants must seek out a wide range of views and encourage dissention, but provisionally suspend judgment of the issue until all beliefs are stated, thereby exhibiting open-mindedness and a willingness to hear multiple perspectives. Tim was extremely open to new ideas and both listened and voiced his own opinions.

The first cultural excursion to Alhambra serves as an example to show Tim's willingness. On the bus ride to Granada, I observed from the body language of the participants that they were apprehensive to participate in the entrance interview. Therefore, I asked if any of the participants was willing to volunteer to be first. Without hesitation, Tim jumped up and enthusiastically offered to be first. Likewise, on the same cultural excursion, Dr. Green informed the students that they had a few hours of free time, but asked if anyone was interested in going to see the cathedral of Granada. Once again, without hesitation, Tim shouted, "Hell yeah!" and then encouraged other participants to go on the tour (very few did). Upon watching this, I wrote in my field notes:

I love [Tim's] attitude toward life. He is the embodiment of Mezirow's willingness that is necessary for transformative learning to take place.... 
Ironically, [he] also [has] the most difficult time with Spanish. It will be exciting to see what happens with [him] and this [study-abroad] experience.

Tim's love of food served as a metaphor for his willingness to participate in cultural activities. In the entrance interview, Tim expressed his love for food and described himself as a "foodie." Moreover, he stated:

I very much use food as an analogy for pretty much everything in life.... Whether its emotions or ... beliefs... everything has its own little flavor. And once you have enough experience with those flavors, you can start to combine them to build interesting dishes.

The following story will both honor Tim's belief that food is a metaphor for all things and show his willing nature to "suck the marrow out of life." During the festivals, Tim got hungry around midnight. The story is best understood in Tim's words:

I drank too much wine at the bullfight. So we went out, and then I got hungry so I split off to go on a solo adventure to go get a meal. And I ended up at [a food kiosk.]... I went there because they had a sign hanging up that said conejo, and I like eating animals I've never eaten before, and so I was like, “Ah, conejo, awesome! I'm gonna get that." So, I got that, and it arrived, and I took a picture of it, 'cause I've been taking pictures of my food.... I really enjoyed it, it was delicious. The next morning, when I woke up and discovered just how intoxicated I had been ... I was kinda like, "Oh, I drank too much.” And then I was looking through my photos of the previous night, and I got to the one of ... my conejo. And I was just like, “Oh my god! That's half a rabbit!" ... And it just made me 
realize that, when I ordered it, I seriously just ... snapped the picture and then ... dove in. I abandoned my fork and knife after a couple of minutes and ... destroyed that thing. And at some point, I remember while I was eating it I got to this ... little dab of cream cheese, and I was like, "Huh, that's...kind of odd. I don't know why it's there, but, it tastes good." And then I'm looking at this picture the next morning, like, "Oh...that was its eyeball.” I straight up ... ate half of that rabbit. I just...destroyed it. Sucked its bones clean. Which, I'm not sure I actually could have done if I'd been ... slightly more aware of what it was I was eating. 'Cause...I picked up what turns out to have been the skull and I kind of thought, like, "this is an odd-shaped bone.” But...I just dove into it. It was awesome, though. It was real tasty.

In the member-check interview, I reminded Tim that he used food "as a metaphor for everything." I asked him if he felt that the rabbit experience was a valid representation of his willingness to experience Spanish culture and food. Tim agreed, saying, "I tell people I went "full barbarian"” on the rabbit. I replied, "You lived that rabbit to the fullest."

However, according to Mezirow's TLT (1996, 2000), Tim should have had multiple opportunities with cognitive dissonance and transformation due to his willing nature. However, he did not experience transformative learning. Nevertheless, Tim's apparent lack of transformation can be understood through the lenses of the following two sections about linguistic "otherness" and personal identity.

What role did encounters with cultural and linguistic "otherness" play in the transformative learning (or lack thereof) of each participant? First, I will describe 
Tim's experience with cultural "otherness," followed by an explanation of linguistic “otherness."

Cultural "otherness." Tim explained in the entrance interview that he hoped for an "immersion experience" and a "completed foundation" of what he had previously learned about Spanish during the study-abroad program. As explained earlier, Tim did not experience any significant cultural shocks, and I observed he did not feel linguistically or culturally "othered" at any time during the study-abroad program. For example, I asked Tim if he experienced "otherness" in the member-check interview and Tim explained:

I did have the feeling ... and, I enjoyed that feeling.... I really enjoyed ... being an outsider. That's kind of an odd thing to love. But, being a tourist ... [is] like being a stranger ... in a strange land. I really enjoyed that experience ... but, again, like, I'm pretty good at going with the flow wherever I am.

Nevertheless, Tim experienced "otherness" from the other participants and the Spanish language. When I asked him about culture shocks, he said, "None, really" thought about it for a second and then said "I would say the language has been the biggest culture shock for me."

From a cultural standpoint, Tim felt he never fit in to the expectations of his host family. Any time Becky or Kynzee (discussed later) mentioned the activities they were doing with their host families, I observed and heard Tim's yearning for a similar experience. I could see he was hoping to go "full barbarian" with immersion in his host family. Nevertheless, his host "mom" had two sons in their early twenties and another 
foreign exchange student living in her apartment. It seemed that the host family environment was much more transactional than culturally immersive, to Tim's disappointment. He commented on this exclusion in the exit interview:

I don't know what my host-family's expectations were. But I feel like I have somehow not lived up to those. But I do largely keep to myself.... I don't know if they were expecting someone who was going to come into their home and ... ingratiate themselves and be a part of the family. But for me, I've honestly stayed more of like a house-guest.... I think when I leave, they will be glad to have their house back to themselves.... Which ... again, is something that kind of disappoints me. But on the other hand, it's just kind of how I roll.... I would say very much one of my ... life philosophies.... But still, [I want] to have as minimal an impact as possible on pretty much everything.

I observed that Tim did not mention his host family after the entrance interview unless I specifically asked him about them. Furthermore, the more distant he grew culturally from the host family, the more culturally enmeshed he became with the other participants. Tim did not know any of the other participants prior to the study-abroad program. Moreover, I observed that the rest of the participants were slow to fully include the single male participant. To be clear, the female participants were congenial and pleasant with Tim, but as one would be with a classmate. Notwithstanding his initial exclusion, I observed that Tim was an integral part of the study-abroad group by the beginning of the second week. However, unfortunately for Tim, the more he associated with his fellow students, the less he spoke Spanish. While the other participants appeared 
to negotiate this English-out-of-class, Spanish-in-class system, Tim struggled linguistically. This linguistic struggle will be described in the following subsection.

Linguistic "otherness." Once again, I do not think Tim ever felt "othered" by the Spanish language. Yet, other participants increased Spanish capacity regularly throughout the program. Alternatively, Tim's observable Spanish speaking and listening capacity observably decreased throughout the study-abroad program. I have reviewed the data repeatedly to ascertain if this was my perception or a change in relative capacity compared to the other participants. However, I observed that his proficiency to both speak and apparent comprehension (as demonstrated by his responses to questions and inclass assignments) diminished each week. I asked Tim in the member-check interview, "The more you spoke English, the harder it seemed [to] transition back to Spanish.... Is that true?" Tim replied without hesitation, "Oh yeah, definitely. It's not really a problem I expected to have.... The amount I use a language is directly proportional to how easy it is to continue communicating in that language."

I wrote in my field notes during the first week of the program that Tim and Kynzee (discussed later) struggled the most in Spanish. Despite a deficit in Spanish capacity, both students were outgoing and participative in class, voluntarily answering questions as much as Jennifer or Kristene the first week. In contrast to Kynzee, however, Tim appeared to have better comprehension skills. The class following the cultural excursion to Alhambra, Juan lectured about the historical significance of Alhambra. As Juan pushed the students to understand and discuss the different aspects of Alhambra more deeply, I discerned that Tim must have paid attention and understood the tour 
guide. I wrote in my field notes, "It is apparent that [Tim] paid ... more attention on the [Alhambra] tour, because the guide definitely talked a lot about the Muslims, the Christians, [and] each of the Spanish kings [who] occupied it. The guide repeatedly showed the influences of the different cultures." In class, Tim was sharing all of the things he learned and Juan was visibly impressed. The other participants recollected very little from the tour.

Later that week, the students were responsible to present on a topic Juan assigned them regarding Spanish culture and history. I wrote during Tim's presentation, “Tim did a good job. It looks like he did some work in looking up [information] that was not in the PowerPoint that Juan gave them. His Spanish struggled, but the information was good." By the end of that week (the half-way point), however, I began to notice a sharp decline in Tim's use of and the proficiency of his interactions in Spanish. I wrote:

Something weird has been happening with Tim and Spanish the last couple of days that further shows the huge disparity between his Spanish speaking ability and his great comprehension ability. He has been trying to explain and then he just "cracks" and starts speaking in English. I wonder if he has given up, or is tired, or even realizes he is doing it.

A few days later, I observed, "[Tim] seemed to understand really well at the beginning.... As he became more included in the "culture" of the [study-abroad] group, he became less able to understand and interact [in Spanish]." By the end of the last week, I observed that Juan was visually and verbally disappointed in Tim's final class presentation, citing that his assignment was to discuss the evolution of modern Spanish 
society, not about populations that had conquered Spain over time. In the exit interview, as we discussed this phenomenon, I asked Tim if he had any thoughts as to why this happened. He opined:

I had wanted to jump into the pool.... It's a zero-entry pool, and I've just been chilling on the slope.... 'Cause, whenever me and the girls hang out, we use English, which...has bothered me since day one, but obviously not enough to be like, “Come on, ladies. Let's use Spanish.” And, I've noticed it has negatively affected me, 'cause after hanging out with them I'll go home and [my host "mom"] will engage in conversations, and...I've gotta get those gears spinning again.... I can feel it take longer than it should.... But then once I had ... the girls as an English safe-haven ... that's probably been my ... largest negative.... I've failed on my immersion checkpoint ... but I've still really enjoyed my time here. In the member-check interview, Tim reiterated the same idea, "Pretty much anytime ... me and the ladies got together, we would get caught up in the ease of communication, so we would... use ... easy Spanish. So, Spanglish.” Nevertheless, Tim did not blame the LU program or Dr. Green for his lack of immersion. Rather, he blamed himself. Tim stated in the member-check interview:

On the university's part ... they did everything right.... I experienced a failure of immersion only because of myself ... and that ... blinds me to...fully dissecting the immersion experience. But so far as I can tell ... I like the way [Dr. Green] did it. I liked him just throwing us into the pool as opposed to...holding our hand.... I think ... the program was well designed as far as immersion goes. 
Furthermore, I asked Tim if his difficulty speaking Spanish impacted the way he saw or treated "strangers" in the United States. He answered, "Not so much.... I'm generally a pretty accepting guy ... so ... I've been ... good at putting myself in someone else's perspective.... Not necessarily putting myself in their perspective, but sort of understanding their perspective."

Although Tim stated he did not feel "othered," his experience aligns with Foster's (1997) findings about the typical anxiety or vulnerability associated with learning another language. According to Foster (1997), this vulnerability sets up an excellent situation for transformative learning to occur. Furthermore, she argues that learning to communicate in a different language is unsettling, causing a distorted view of self. However, Tim never described feeling disoriented, unsettled, or had his self-concept or self-competency challenged (Mezirow, 2000). He never relinquished his view as a competent communicator, and therefore, never had an opportunity for a liberatory transformative learning experience (Goulah, 2007). Ironically, it was precisely Tim's "go with the flow," or willing attitude that prevented him from experiencing the immersion and transformation he sought from this study-abroad program. Consequently, I will discuss in the Discussion chapter my finding that Mezirow's $(1996,2000)$ use of the term willingness is unfocused and in need of further empirical analysis.

What is each student's racial, economic, gender, and cultural identity? How does each student's identity impact their worldview and their transformations (or lack thereof) during travel? Tim was a White, heterosexual, able-bodied male, who self-identified as politically liberal. As stated in the introduction, Tim was twenty-eight 
years old at the time of travel. He had traveled throughout the United States Midwest, Rocky Mountains, and Southeast regions, and California. Moreover, Tim had been to Mexico twice on short mission trips with his church group. In his early twenties he left his Midwestern state to live in Colorado and Utah for a year and a half. Apart from those travels, Tim spent most of his life in the poorest county in his home state, in the town where PSU was located. Students used a "mildly derogatory" or "playfully derogatory" (Tim's words) term of "townie" for people from the area where the university was located.

During the entrance interview, Tim informed me he "always wanted to travel abroad." He liked his Spanish classes, and said the study-abroad program "seemed like a natural fit." However, Tim had the hardest time of all of the participants earning enough money for the study-abroad trip. In the entrance interview, he informed me that he wanted to cancel his place in the study-abroad program about two months before it started. Notwithstanding, Dr. Green encouraged Tim to keep working toward Tim's goal of paying for the trip, which Tim was eventually able to do. Although Tim said he never lacked food, clothing, or shelter growing up, he did say several times throughout the study-abroad program that his mother never ate much while he was growing up (he also worried often because he did not see his host "mom" eating). Initially, this information led me to believe Tim grew up working class. Nevertheless, I found out in the exit and member-check interviews, although his mom was divorced when Tim was very young, she was a certified public accountant, and he felt that "middle-class child of divorce" was a good representation of his class upbringing. However, like many middle-class students, 
Tim was solely responsible for college expenses, and, therefore, paid for the study-abroad program from funds he earned himself.

Often throughout the study-abroad program I tried to understand the seemingly contradictory intersection of Tim's willing nature and his lack of transformation. It did not appear to make sense from everything I had read about transformative learning and study-abroad. In the member-check interview, Tim and I discussed his lack of transformative experiences. He felt that his age and gender played a "big factor" in his lack of transformative learning, which will be discussed below in the Age and Male Privilege subsections. Furthermore, I hypothesize that Tim's "smooth sailing," liberal nature might be seen as a post-racial or colorblind attitude through a critical race perspective lens in the final section on Race Privilege.

Age. As I analyzed Tim's data, I consistently thought that being twenty-eight years old might have had an influence in his lack of transformative learning. Combined with his easygoing temperament, I perceived that many of the experiences the nineteen to twenty-two-year-old participants were going through, may have happened to Tim at a similar age. After all, he had lived outside of the house, and had traveled to a place completely novel to him when he lived in Utah and Colorado for a year and a half in his early twenties. I wondered if much of the cognitive dissonance the female participants felt on the Spain trip might have happened to Tim during his move to Colorado and Utah. I asked Tim if he felt his age or life experiences may have played a role in his lack of transformative experiences in Spain during the member-check interview. Tim responded: I do think my age plays a pretty big factor....You know that saying that "you 
can't teach an old dog new tricks." I can feel myself turning into an old dog.... I can feel my brain closing to new ideas. Which really bothers me. But I'm utterly unsure of what to do about it.... The whole trip back, from Levante back home, the closer I got to home, the more relieved I became. Not relieved, but like a combination of relieved, relaxed, happy to be going home.... But I was also ...'unhappy' ... [because] I could ... feel myself already falling back into some of my old habits.... Like I could feel myself ... putting on old gloves, an old costume, putting on an old id ... my American identity ... my home identity. I could sorta feel myself putting that back on and it filled me with ... [a] growing tinge of sadness of...returning to the status quo.... But again, that was tempered with my relief at getting closer to my own bed, and my own shower, and my own laundry.

Tim went on to explain that returning to his hometown after living in Colorado and Utah was more of a culture shock than going to or coming home from Spain. He said, "That was actually more of a culture shock than coming to Spain.” Consequently, I hypothesize that many of the "otherness" and cognitive dissonance other participants experienced was experienced by Tim previous to the study-abroad trip to Spain.

Male privilege. According to Ross (2010), one of the keys to second language learning is a learner's willingness to "be like representative members of the 'other' language community, and to become associated, at least vicariously, with that other community" (p. 37). While Tim was "full barbarian" or "Hell, yeah!" about experiencing the local culture, I never sensed a desire to "be like" them. For example, this was 
especially evident in Tim's attire. While many of the female participants were concerned about how they dressed and to avoid "looking like tourists," Tim's manner of dress and overall comportment never changed from his "American identity." Tim wore long, baggy cargo shorts with plaid, cotton, short-sleeved shirts every day. In contrast, I observed that Spanish young men Tim's age rarely wore shorts. If they did, it was to exercise or to go to the beach. Moreover, I also never saw a man in a plaid, cotton, shortsleeved shirt. Even for events where the female participants would dress up, Tim often wore casual clothes. When pressed by the female participants about his attire, Tim would often say things like "I am who I am." While I admired this quality of self-confidence in Tim, I also noticed that the female students sensed an intense cultural pressure to dress more representatively of Spanish women.

Several other examples serve to point out Tim's gendered worldview. He often used gendered speech like "That's how mothers are" (compared to his host "father") or calling the female participants "ladies" or "girls." Furthermore, Tim was very protective of female participants. When they went out dancing or drinking, Tim would often physically remove (in a non-combative manner) men dancing too closely to the female participants. However, it must be noted that several of the female participants told me they enjoyed that aspect of Tim (but it does show a gendered worldview, nevertheless). One of the female participants recollected fondly that Tim "pretended to be my boyfriend" so other males would leave her alone during the festivals.

Another aspect of a male privileged worldview was Tim's tendency to see his viewpoint as factual. This aligns with hook's (1994) observation that male students often 
dominate discussion, perceiving their viewpoints as universal or factual. In classes and other discussions, I observed him explaining things from his viewpoint, in a matter-offact way. He did this with nudity in Ana's art class with phrases like, "It's Europe, so I was kind of expecting it." Furthermore, Tim's viewpoint often supported the Dr. Green and the universities, while the female participants were more prone to voice criticisms. For example, in the focus-group interview, Jennifer complained that anytime Dr. Green's was confronted with lack of involvement, he said "it's all part of the experience." Jennifer expressed that she felt this "copout" was especially problematic in getting the students from PSU to LU. Tim interrupted:

I don't think it's a copout, by the way. Early on, like pretty much the second I landed in Madrid and realized ... [Dr. Green] does all of it on purpose. He purposely throws us into the ocean. You gotta swim on your own ... it's how you learn to swim.

Nevertheless, it is important to point out that Tim's liberal philosophy supported equal rights, pay, sexual/marital freedoms that were often discussed in Juan's and Ana's classes. Nevertheless, he was raised in a patriarchal society and had adopted habits of his surrounding culture. Consequently, Tim was unaware of how cultural influences reinforced (or even formed) his patriarchal frame of reference.

Racial privilege of liberal Whites. A recurring theme in CRT scholarship is a distrust of liberal agendas (Litowitz, 2009). Ironically, I observed that Tim's liberal nature, reinforced with a lifetime of unacknowledged socio-cultural privilege, created a situation where Tim could "go with the flow" in "smooth sailing" without being faced to 
be challenged and grow toward social action. Societal institutions of patriarchy and Whiteness allowed Tim to escape marginalization and victimization that many of the female students feared and experienced throughout the study-abroad program (JohnsonBaily \& Alfred, 2006). It was precisely due to Tim's racial and male privilege that he could say, "I feel like I adjust very well to new things. I feel like you could drop me anywhere." Furthermore, because it is almost impossible for individuals in privileged positions to see the flaws in their own frame of reference (Brookfield, 1998), it was possible for Tim to have liberal values while simultaneously escaping daily indignities that marginalized groups encounter (Leonardo, 2009).

The myths of colorblindness and post-racial society are ideas that are maintained through the "psychological and material benefits from an unearned position in society" (Leonardo, 2009, p. 273). Thus, Tim's repeated "people are people" narrative a comforting myth that parallels colorblind, liberal approaches underpin a normalized worldview of institutions that reinforce hegemony (Gillborn, 2009). Likewise, Tim's failure to fully acknowledge the differences in experiences between himself and other participants aligns with the idea of a post-racial society (Tatum, 2009). I found Delgado's (2009a) critique of slow-moving liberal reforms appropriate to Tim's lack of critical selfawareness. In a deliberate re-phrasing of Delgado quote in the literature review, "liberals and moderates lie awake at night, asking how far they can take [their liberal philosophy] without sacrificing innocent white males [worldviews]" (p. 110).

As stated in the literature review, it is important to note that there is a difference between being a White person and the social construction of Whiteness (hooks, 1994). I 
observed that Tim was kind and respectful to everyone he met, as is essential to the humanist theoretical underpinning of TLT (Baumgartner, 2012). However, I feel that Curry-Stevens' (2007) version of "pedagogy of the privileged"-wherein White learners learn about racism, men understand patriarchy, and ... both upper- and middle-class learners understand neoliberalism and class exploitation—was missing from Tim's studyabroad experience. In the Recommendations for Further Study section in the Discussion chapter, I will outline suggestions for creating a counterhegemonic form of travel for transformation in which students who have liberal values like Tim (as well as Kristene or Jennifer) may be taught how to understand and acknowledge their privilege and then work to disrupt oppressive forces that are within their sphere of influence.

\section{Participant Six—-Kylie"—From Tourist to Advocate through Linguistic "Otherness"}

Kylie was a twenty-year-old student between her sophomore and junior years of college during the study-abroad program. She was from the wealthy suburb that Kristene and Anne were from, but she was quick to point out that she was not the archetypical wealthy suburbanite that was characteristic of that area. Indeed, during the membercheck interview, Kylie indicated that because her dad went through a series of layoffs and career changes while Kylie was in middle and high school, Kylie felt like she identified as a lower-middle class student. She was attending school on a Pell Grant, yet she also acknowledged that she was raised in a wealthy suburb and had the benefits of great schools and extended family financial support that is common among middle and uppermiddle class students in the United States. 
Within the first couple of days of classes, I repeatedly observed that Kylie never talked in class unless called upon by the professors. On our first cultural excursion I asked her if her quietness in class was Spanish-language related. Kylie informed me that she simply did not talk in large groups whether it was in English or Spanish. Although she did not talk in groups, Kylie was very open and talkative in one-on-one settings. Consequently, I found that formal and informal interviews were extremely beneficial for eliciting thoughts from Kylie. Furthermore, although Kylie spoke very little in class, she invited me to follow her blog posts about her experiences in Spain. These blog posts provided information that was extremely useful for interviewing Kylie. Moreover, Kylie and Tim were the two primary participants in the Flickr data collection method (the two of them easily provided $90 \%$ of the photographs). Their pictures gave me insights into the lives of the participants (minus Kristene to whom I promised I would not look at photos of her) outside of class and spurred many questions for all of the students during interviews. Kylie's shyness during group settings also caused me to make some in-field data collection changes that are common in qualitative research (Marshall \& Rossman, 2011). For example, during the focus-group interview, I observed that Kylie did not say anything. Therefore, I asked her if I could interview her about the same questions the following day, which she happily agreed to do. In that interview, as in all of our one-onone interviews, Kyle was very open and transparent about her thoughts.

I kept Johnson-Bailey and Alfred's (2006) critique of Mezirow's (2000, 2012) model of TLT mentally present as a researcher as I studied Kylie (and Anne). In the literature review, I cited their claim that Mezirow's model largely ignores and fails to 
fully include traditionally quiet classroom members (Cranton \& Taylor, 2012; Tisdell, 2012). Consequently, the researcher's responsibility, according to Johnson-Bailey and Alfred, is to ensure that quiet class members have full access to and fully participate in class discussions. It was due to their research that I made in field changes to the data collection in order to give Kylie space to speak when I could see that she did not during class or focus-group interviews.

One other component about Kylie is fundamental to understanding her studyabroad experiences. One of Kylie's childhood friends, "Amy," came to the study-abroad program as well. Whenever Amy and Kylie were together, they talked and laughed plentifully. I chose to exclude Amy from the research because she attended a different university in the United States, attended a different study-abroad program at LU, and only participated in the cultural excursions with the PSU participants. Because Amy's classes met in a different building at the same time as the PSU students, I felt that the time I spent observing Amy would take away time from observing the other eight participants. Furthermore, I did not write about Amy's program in the research proposal. Through an exchange of emails with the chair of my dissertation committee discussing the pros and cons of including Amy, I decided to exclude her from the findings in this study. Nevertheless, although Amy was not studied, she was an integral part of Kylie's experience in Spain. However, Amy will only be mentioned as talked about by Kylie from this point forward. In sum, Amy will appear as constructed by the thoughts and words of Kylie, not as a participant.

Prior to the study-abroad program, Kylie had traveled extensively throughout the 
Midwest, Southeast, and Rocky Mountain regions of the United States. The study-abroad program was Kylie's first experience traveling outside of the United States.

To what extent do seven students experience the elements of transformative learning during a month-long study-abroad experience in Spain? Similar to other participants described previously, I will discuss culture shocks, adjustments, and surprises Kylie experienced. In contrast to previous participants, I will discuss Kylie's self-identified transformation in the following section on linguistic and cultural "otherness" because her desire to be an advocate for English learners in the United States must be understood in the context of Kylie's perceived "othering."

Culture shocks, surprises, and adjustments. As with many of the other participants, Kylie experienced both culture shocks and cultural surprises, which are outlined below.

Culture shock: toplessness and affection. Similar to many of the female participants, Kylie experienced initial culture shock related to women at the beach who wore only bikini bottoms, and at the displays of physical affection in public spaces. In her blog, Kylie addressed a discussion in Ana's class:

One thing we mentioned was that people are a lot more "affectionate" here, you see them kissing in public with a lot of physical affection. Ana, my teacher, told us that if a couple were kissing on the bus, it would seem normal to her. The awkwardly high number of topless women on the beach is also normal and acceptable to her, she was actually a little surprised when we told her that if you were to do that in the [United States], you would be added to the registered sex 
offenders list.

In the member-check interview, Kylie added that the practice of giving besitos was uncomfortable to her because she was "not a very physically affectionate person." She explained:

And so for me, like having to be in close contact with everyone ... as soon as I met them ... [and] touch their cheek.... There's (sic) people who I've known ... since ... elementary school, and I probably haven't ever touched their cheeks. Don't really ever care to ... So that was kind of weird for me.

As the program went on, Kylie became accustomed to the nudity, but not the physical affection. She commented on this in the member-check interview:

As time went on it was less surprising. It was just ... like, "Ew, look at that one over there. Nasty!" ... At first it was extremely shocking. Like the first time I went to the beach and saw someone there, I was like, "Oh my God." But then, it kinda became a norm.

Cultural shock: Homework. Similar to Jennifer, Kylie voiced frustration with the amount of homework she had to do in the LU classes. In the entrance interview she said: I've been a little disappointed with the amount of homework we've had in one of our classes. It's been kind of...frustrating.... I understand. But at the same time, I'm like, 'I'm in Spain! I really don't want to do anything. I'm just taking classes out of obligation." I just want to ... go out and explore, and be part of the culture instead of a student, but ... that's just life..... [The classes are] not hard, it's just a lot of little things that end up piling up, or things that I have to talk to my host- 
mom about, so I can't do it until after dinner, which is like 10:30, and by that point I'm like, “Okay, like, I want to shower, I want to go to bed, I don't want to do homework." And so, it just throws my whole schedule off.

Furthermore, Kylie wrote in her blog, "What is the point of having two classes over Spanish culture if I have so much work to do that I don't actually get to experience the culture?" Kylie reiterated a similar sentiment about the exchange between learning about the culture and experiencing the culture in the member-check interview: It was kind of that option of ... do I go out and really experience this culture, or do I spend time in my culture class where I'm supposed be learning about culture, by doing homework and not experiencing the culture. I thought it was just kind of like, ironic.... 'Cause our class is where you learn about culture, but if you don't have - like, the best way to learn is to emerge [sic. immerse] yourself. And, we were kind of prevented from doing that.... There was a lot of homework. After the final exam, I saw Kylie talking to Ana about her grade. Ana pulled me aside and asked what Americans expected when they took essay tests. I asked her what she meant. She explained that Kylie wanted partial points on one of the test questions in Ana's exam. The question had a painting from a famous Levantino artist. The short essay question asked the students to name the artist and describe the significance of the painting. Ana told me Kylie's response was "This is an important painting by a Levantino artist." Ana exclaimed (the students were gone at this point), "James the class is called Levantino Art and Culture. Of course it is an important painting by a Levantino 
artist!" She said that Kylie expected some points for answering, even though the explanation was obvious.

Following that exclamation, Ana said that she was disappointed that the PSU students were concerned about "what was going to be on the test" and "what the right answers were" more than they were concerned about the poets, authors, painters, sculptors, and architects discussed in the class. Ana explained that she wanted the students to react to the piece of art as human beings, not as students. Additionally, she wanted the students to fall in love with the artists, not merely pass the class.

Furthermore, she expressed disappointment that she had told them where many works from the artists they studied were located throughout the city, yet none of the students stated they went to see them when Ana followed up.

Cultural shock and surprise: Food. More than any other topic, Kylie talked about food in her blog and in interviews. The change in diet occupied both a fascination and shock simultaneously. Kylie's first culinary cultural shock came when her host "mom" made a Spanish style pizza during the first week in the study-abroad program. Kylie explained that her mom was lactose intolerant, so the pizza had no cheese. However, it was the other toppings rather than the lack of cheese that shocked her. In the entrance interview Kylie explained:

Okay, pizza without cheese, like I'll deal with it...." But, she had me put tuna... and hard-boiled eggs... and these things that are kinda like pickles but they're round and they're more like, I don't know, they're from a little tree, I don't remember what she called them.... And something else, like some sort of 
like canned meat... And, it was ... literally the nastiest thing I've ever eaten. Furthermore, Kylie was shocked when the pizza reappeared as dinner the following evening. She said:

We had leftovers ... of the pizza.... And she didn't even put it in the refrigerator; she just like stuck it in the oven, and then tried to feed it to me the next day for dinner... I was like, "Yeah, yeah, I'm good."

Kylie went on to explain that not only was the pizza gross the first time, the fact that the meat and eggs stayed out of the refrigerator for a full day was extremely unappetizing for her. Consequently, she chose not to eat dinner that night.

Another instance that negatively impacted Kylie was when her host "mom" used rabbit, instead of chicken, in a paella she made. Kylie recounted the incident in her blog: I told her that I would try rabbit, but would like to have some chicken as a back up just in case I could [not] do it. I told her that I had a slight conflict of interest because I had one as a pet for over seven years... She thought that was kind of weird. The paella was really tasty, but I'm still not completely sure if [my host "mom"] really did make half of it with chicken or if she was just tricking me...It was the toughest chicken I had ever eaten and flavored with a lot of Rosemary. It is also important to note that Kylie discussed positive experiences with food in interviews, classes, and her blog. After a week of the study-abroad program, she reported, "I'm still dealing with some adjustments to the food here! I really enjoy it for the most part. I eat fish most days of the week and when it isn't fish, it is chicken. Those are two things I love!" In addition, during the entrance interview she said, "So far food 
hasn't been too bad here, other than the tuna pizza my host mom made for me a few nights ago." Furthermore, in her blog she explained, "I got to try gazpacho for lunch as well as some sort of fish that is in the same family as a shark!” Additionally, Kylie described a mild transformation in taste regarding olives in her blog:

Olives are eaten here like Americans eat Lays. It is insane. Before coming here I really didn't care much at all for green olives. Now that I have been here for just over two weeks, I can eat them like candy!

Moreover, Kylie overcame a fear of trying anchovies. She stated, "The anchovies were finally served the other night! I was surprised, they weren't nearly as bad as I thought they would be."

Lastly, Kylie expressed fascination at the items that Levantinos found exotic. In her blog she described an experience in Ana's class discussing food:

Today in Ana's class we talked about foods that were exotic to Spain: hot dogs, corn on the cob, milk, cows, and peanut butter... Cows are exotic animals to them. Cows are like camels to us.... The cultural differences between the two countries amaze me every time we talk about them.

In the member-check interview, Kylie said that she still misses the olives and loquats in Spain.

Cultural adjustment: Transition to apartment living. At the end of the first week of classes, Juan asked students what surprised them most after living a week in Spain. When Juan called on Kylie, she said she thought that everyone would live in little white houses with tile roofs like are often portrayed in travel brochures. Furthermore, she 
discussed the transition to apartment living in the entrance interview: "I live in an apartment-type thing, and I've never lived in anything like that before, but it's kind of cool." In her blog, she discussed loving the hotel shower in Granada because she could take a shower that was longer than she could take in her Levante apartment:

"I took a longer shower than usual because it was just so amazing to have hot water for an extended period of time! At my house I can get a few minutes (maybe two or three) before it gets freezing cold. That is one thing I really do miss about being home!"

Nevertheless, this experience was more of a cultural adjustment than a culture shock. However, in the member-check interview, Kylie said she got used to apartment living after about a week of living in Spain.

Cultural adjustment: Walking "though" people. Although Kylie really enjoyed that "people walk everywhere," her timid disposition struggled with "people... walk[ing] through each other." In the entrance interview she said:

[People] don't...part.... At home I feel like, “Oh, there's someone here, so we're going to go over to the side," but here they're just like, "Oh yeah, let's just weave through." Not a big deal. No one really gets mad or upset.... If someone bumps into me at home, I'm just like, “Creep!” But here, it's not really that way.

Cultural surprise: Beauty of Spanish landscapes and architecture. Kylie was an avid photographer (and Flickr uploader). Kylie was a little more prone to photograph the participants, while Tim was more likely to photograph architecture (however, both participants photographed both subjects). Nevertheless, many of Kylie's photos and blog 
posts were about how much she enjoyed viewing the architecture and landscapes of Spain. Regarding the LU campus, Kylie blogged, "The campus of the Levante University is beautiful and huge." Furthermore, about the cathedral in Granada, Kylie wrote in her blog:

The church was so beautiful! I will make sure to attach photos of this in the very near future. The actual church part was incredible. I can't imagine going to church somewhere that is not only huge, but also so beautifully distracting. I would never pay attention to the homily because I would always be focusing on the art in the sanctuary.

Likewise, about Alhambra, Kylie wrote: It is one of the oldest pieces of Islamic architecture, originally built in 889 and reconstructed in the 11th century, and housed the last Muslim emirs in Spain. Our tour was three hours long and we walked nearly 5 miles! It was one of the prettiest places I have ever seen though.... I don't even have words to describe that castle, it was just magnificent! There were these amazing gardens, I wanted to stay and take pictures of everything. Real life application — a picture is worth a thousand words.

About the drive to Granada, Kylie wrote:

The drive was very pretty; there were lots of little towns with white houses and even some towns with houses built into the side of a mountain. I'm sure that people who make drives with similar scenery would think that we were just seeing common sights, but for me it was fascinating. I am just so amazed about 
how different it looks here compared to [the United States].

Moreover, Kylie found it visually stunning how close the Mediterranean Sea and the mountains were. In the entrance interview, she informed me, compared to the rolling hills and flat landscapes of the United States Midwest, Spain's topography "amazed" her.

Cultural surprise: Festivals. Kylie called the fireworks during the festivals "one of the most thrilling and exciting things in my life" and stated that she could "feel [her] arm hair moving with the sound waves and [her] ears [ringing]" in her blog. In the exit interview, she stated that the fireworks "were really impressive. Fourth of July is going to be a little lame now."

Moreover, she also expressed excitement that the "whole city goes crazy" for the festivals. In her blog she explained:

A lot of the main streets are basically shut down and have tents set up with restaurants and little shops, some with really cool portable storefronts.... The whole town is alive! The streets are full of people at all hours. During the festivals, people do not go to bed before four-that includes the small children! Last night (Friday) there were little boys running around lighting of firecrackers at 2:00 in the morning! It seems crazy to me, but [my host "mom"] assures me it is not. There are random street performers making music at any given time of day and streets shut down because they are reserved for dancing! How insane is that? The whole town is a party! The nightlife is really something else. Soon most of the stores will be closed and the entire town will be celebrating. At random points in the town there are these giant sculptures that artists have been working on for 
this festival. On Tuesday all of them will be set on fire! Pictures to come! Cultural surprise: Bullfight. In contrast to many of the other participants, Kylie was surprised that she enjoyed the bullfight as much as she did and was frustrated with others who did not. In my field notes, I observed that Kylie did not appear disturbed at all. I asked for impressions and she said, "eh." This was immediately after hearing Anne rant about the injustice of the bullfight for about five minutes. I wrote, "Kylie has family with a cattle farm and has witnessed animals die.... She said that she feels that they are going to die one way or another. She also noted that she felt that she should feel sad." Kylie further commented that she thought the bulls may have it better in Spain because they are free range, grass fed animals until the final event. In the exit interview Kylie stated:

[The] bullfight ... was exciting to watch. And honestly, personally, I think it's better than ... the way they do it in the U.S. Like, sure it's kind of gross watching [the bull] ... die. But I think the life that bull lives is a lot better than the life cows live in the U.S, like being penned up forever so they stay tender, and then ... walking in a line and then just ... offing it. I think that's horrible. So where the bulls ... get to live free-range without much interaction with people ... I'd rather die that way.

Additionally, in her blog, Kylie commented on the spectacle of the bullfight: I actually found myself enjoying watching the fight! The final moment of the bull's life was a bit hard to watch, when the toreador finishes it off by jabbing it in the brain several times with a dagger. I had to laugh at how cocky the toreadors 
were, they really do think they are hot stuff! I know this is horrible, but I kinda wanted at least one of them to get a bullhorn in the butt (don't worry, one did).... Some of the girls in my group really freaked out about the fights and were even angry about it, leaving at the intermission. Maybe I don't have a heart, but I honestly think these bulls have it a lot better than a lot of cows in America do.... Maybe I'm crazy, but who has it better? One girl that was angry about it told me that she thought they should put the toreador in a ring with six bulls so he can have a taste of his own medicine. I think that is so wrong! This girl eats meat. What does she think happens to it before it is put on her plate? People like this make me frustrated. After the toreadors have a victory, they cut off the bull's ear (sometimes both of them, a tail, and a hoof) and do a victory lap while all of the people scream and wave white flags. A lot of people throw flowers or hats at the toreador in appreciation, but one lady threw her panties and a courageous elderly person threw their cane down into the ring! I thought it was pretty funny! One of the toreadors, the best of the three that fought, wears an eye patch because he lost an eye in a previous fight. Personally, that is where I would draw the line!

In the member-check interview, Kylie reaffirmed the thoughts expressed in her blog and in other interviews.

Other: Politics. Throughout Kylie's blog she expressed fascination about the abdication of the king, unemployment, the "corrupt" Spanish government, and that "it's sadly ironic that they eat so much here when, only eight miles south of Spain, there are starving African children! Spain could spare some food!” Although she discussed the 
events happening in Spain at the time of the study-abroad program, I observed no shift in her socio-political stance. Additionally, I asked Kylie if any of her viewpoints about politics or society changed in both the exit and member-check interviews. She stated that they did not. Moreover, while Kylie was quick to notice the disparity between Spain and "Africa," she did not ever mention the socio-political disparities between the United States and Mexico or the disparities within United States cities. Her observations were objective and not subjective in nature (Mezirow, 1998). Nevertheless, what makes Kylie's experiences stand out from the other participants is that Kylie experienced a shift in social action without an observable or stated reframing in socio-cultural epistemology (objective or subjective). Her experiences align with Johnson-Bailey and Alfred's (2006) observation that marginalization may be a more powerful transformative learning catalyst than any other TLT component. Because Kylie's shift in social action is closely linked with her experiences with linguistic and cultural "othering," I describe them in the section below.

What role did encounters with cultural and linguistic "otherness" play in the transformative learning (or lack thereof) of each participant? As I analyzed Kylie's data, I found it impossible to tease out Kylie's cultural and linguistic "othering." It seems that both were rooted in her reluctance to speak in social situations. In contrast to previous participants, I will treat both forms of "otherness" together for Kylie. In addition, at the end of this section, I will explain how Kylie's transformation toward linguistic advocate was rooted in her encounters with linguistic and cultural marginalization, rather than a socio-cultural political shift in epistemology. 
During the first week of classes Kylie found it difficult to transition to speaking Spanish exclusively. She stated in her blog:

Everyday my Spanish has improved a lot! The first day I was here, I was shocked because comprehension was just horrible. Everyday I understand more of what my host mom is saying as well as the people on the street. I really hope this continues to improve!

However, she told me in both the exit and member-check interviews that the shock did not last long. Additionally, in Kylie's first presentation, I noted that her reticence to speak in social situations had an impact on her speaking proficiency. I observed: Kylie's Spanish shows her shyness a little. She has better grammar than the previous two [participants]. However, you can tell from the pronunciation that she does not speak a lot. Her personality, however, comes out a little in this presentation. She prepared extra photos in her iPad and looks outgoing as she presents. This is the first time that I have seen her speak/come out of her shell when she was not with Amy. What is interesting to me is that she does not talk in class or on the excursions (except with Amy on the excursions), yet she seems perfectly comfortable presenting in front of the class.

When I asked why she decided to participate in the study-abroad program, Kylie answered:

I really wanted to improve my ... Spanish-speaking abilities in ways that I didn't feel like normal class could, so having the opportunity to speak to native speakers 
... for an extended period of time, without really any lifeline, seemed like the best way to do it.

On the bus ride home from Granada, Kylie pulled me aside and said she thought of two changes that had happened after a week in Spain. She maintained that she felt that she understood Spanish much better. Secondly, Kylie felt she did not "stick out" so much, meaning that she can walk down the street and not look so much like a tourist. From what I observed of her personality, not drawing attention to herself must have felt like a relief. Several times Kylie commented on not liking negative attention. In the entrance interview she stated:

I'll be out in public ... speaking English with my friends.... Obviously we don't blend in very well when we do that, but... [we] also ... get some dirty looks sometimes, which ... really surprised me. I didn't really think that would happen. But it's happened a couple times.

Likewise, she also explained this desire to not be seen as "other" in her blog: I've started to notice that people don't always react pleasantly to my friends and I when we are on the street and speaking English to each other. We actually get a lot of dirty looks... I don't know if it is because we are speaking in a foreign language or because we look/sound like Americans, but it is kind of weird.... Being a foreigner is a very strange experience! In her blog, Kylie recounted an experience during her second week that set the tone for her feeling of linguistic and cultural "otherness" for the rest of her study-abroad 
experience. Her host "mom" invited Kylie to a Spanish-style barbeque out in the country. Kylie's host "mom" was extremely rude to her during the party. Kylie wrote:

The whole time I was barely acknowledged. When we finally got to the apartment, one of [my host “mom's"] friends greeted me and said hello, but asked [my host "mom"] what my name was (because I clearly couldn't have told her) and responded with, "Keelee?"

[My host "mom"] acted absolutely disgusted.... The birthday girl was very nice to introduce me to her friends upstairs ... but made me say my name because she could not.... When I went back downstairs to the kitchen, I walked in on a conversation between [my host "mom"] and [a] friend ... about me. The lady was asking [my host "mom"] what the heck my name was about.... Just from the tone in her voice I could tell that [the host] was not interested in me being there in the least.... Not paying complete attention to me, the lady asked [my host "mom"] one more time how to say my name and crinkled her nose when she said "Keelee." This time I made sure to pronounce my name the correct way, with emphasis on the "Y." She made me write it down, but looked at it like I had just written an obscene word.... [Throughout the afternoon] my host mom seemed too interested in being with her friends to even think twice about me. I maybe had 5 minutes of conversation with her the whole time I was there. People would talk to [her] about me, in front of me, and look stunned if I tried to answer their questions. If I would try any food or try to say something, they would all giggle.... At one point, [my host "mom] commented that "poor Keelee" has to get up early 
in the morning to go to school.... After six hours of sitting and being excluded from all conversation ... we got home a little past 1:30. I was so frustrated.... [I] felt so disrespected that I wanted to cry. The whole night I was ... treated like a dog being fed table scraps.... My eyes have definitely been opened up to how I have felt about any international student/person I have know[n].

As I analyzed the rest of Kylie's cultural and linguistic experiences in Spain, it seems like this moment was a turning point. Previous to this experience, Kylie seemed to desire to be included linguistically and culturally. She even included exciting linguistic successes in her blog. However, following this experience, I observed (without knowing about this experience) that she ceased attempting to speak Spanish when not in class. Several of the other participants commented that Kylie (and especially, Amy) refused to speak Spanish outside of class. I believe that this marginalizing experience was a point in which Kylie ceased trying to be like the host culture, which is considered an important component of travel for transformation (Ross, 2010).

Following this barbeque experience, Kylie's comments take on a touristic, voyeuristic nature of seeing the "other," rather than a desire to blend in. She began referring to people from Spain as "natives" and "these people." She seemed to identify her host "mom" as the essentialized "other" and appeared to have transferred her host "mom's" personality traits were those of every Spanish citizen. After this incident, Kylie recorded in her blog: 
Well I'll say with my host family it's been, eye-opening. Different. Shocking, I guess, in a different way. Not culture shock, just... Now that I'm past the culture shock, knowing the, like, "Okay, these are how people really are." In addition to the barbeque, a series of miscommunications, microaggressions, and other negative interactions followed between Kylie and her host "mom." For example, her host "mom" refused to give Kylie directions, but gave them to Jennifer instead. Kylie explained during our informal interview following the focus group interview:

I feel like [my host "mom"] kinda thinks I'm stupid.... When we were going to the theatre to meet... I asked her ... "which street do I need to turn on?" She's walked me there before, but I just couldn't remember exactly where it was. And she just looked at me like I was such an idiot.

Additionally, during the festivals, Kylie and Amy came to the apartment to use the restroom. She knew that her host "mom" was still awake, but her host "mom" seemed offended that she came home to use the restroom late at night. Kylie narrated the experience in the exit interview:

[My host "mom"] comes up to me and she's like, "Kylie, it is way too late for you to be coming home just to use the bathroom. If you're coming home, you just stay home. You can't just swing by." And like yelled at me! And I was just like, "I'm sorry, I would have used a public place but I didn't know of any, and she was kind of in an emergency situation." And...my host-mom's like, "Well, I'm sure there's (sic) plenty of public places." 
In addition, there were multiple other experiences where Kylie's host "mom" made comments like, "Kylie! You've been here for almost a month and you don't know what that is?" And, "I am so worried about how your exams went, because...if you don't know what that is, like, obviously you didn't do well." Moreover, Kylie's host “mom” would invite friends over and give wine to everyone except Kylie. Subsequently, Kylie's relationship with her host "mom" grew more toxic the longer the study-abroad program continued.

One other aspect of Kylie's experience in Spain that I observed while reviewing her data is that the words "nice" or "sweet" were often in connection to someone who spoke slowly or spoke English. For example, during the first week she maintained that both of the LU professors were "very sweet" because they spoke "slow[ly] enough to understand very well." Moreover, her host "mom" invited a friend over for dinner and Kylie mentioned that he was "nice" five times and every time she did, within a sentence the phrase "he's fluent in English" accompanied the "nice" qualifier. In addition, Kylie repeatedly associated success with English speaking. For example, a friend of her host "mom" who came over to dinner had his "stuff all figured out" because he spoke English well.

I believe experiencing marginalization (Johnson-Bailey \& Alfred, 2006), combined with Kylie's epistemic stance that "niceness" comes from speaking a language somebody else speaks, led to her biggest transformation. Kylie developed a desire to be more understanding of and linguistically "nice" toward English learners in the United States. In her blog, Kylie stated: 
Coming to another country and now having a name that is equivalent to names like Rishit, Raul, Apu, or Ng in the [United States] is a completely eye opening experience. This whole experience of being surrounded by people that cannot speak the language that I think in is terrifying and exciting at the same time. It is terrifying because if I don't know something, help is hard to find.

Furthermore, Kylie informed me in the member-check interview that several months after the study-abroad program a coworker was rude to a Muslim family from the Middle East who came into a store where Kylie worked. The coworker refused to even acknowledge the presence of the family in the store and made pejorative references to their nationality and religion after the family left the store. In the member-check interview, Kylie explained her reaction to the coworker's discrimination:

I could feel [the oppression] in the air when the people where there.... I'm sure [the family] could feel. 'Cause it got really quiet when they walked in.... I was more... sensitive to it.... I was like, "I've been there, been that." You're in the United States, you need to embrace it. We are a place of multiple cultures, you can't just turn it off and like block them out because you don't believe in it. I thought it was ridiculous.

After telling me a couple of other stories of watching English learners in her college classes get discriminated against (one was from improper reading of the students' names and the other was from a professor not even trying to be linguistically relevant/inclusive), Kylie expressed a desire to be more inclusive: 
I think ... since going to Spain ... I'm more sympathetic with people ... if they can't speak English, or ... they just don't fit in.... “Okay, I get it. I've been there." Like I understand you don't always know what to do with stuff. Give 'em time. Someone direct 'em.

In sum, Kylie experienced a transformation in how she perceived linguistically and culturally marginalized "others" in the United States apparently from merely experiencing “otherness" herself. However, Kylie's shift in action was not tied to a stated or observed shift in socio-political frame of reference. Rather, the shift was linked to her perception of "niceness." Once again, I will state with Kylie what I have with several other participants that a limitation of the framework of this study is recognizing that socio-cultural subjective reframing may happen much later than the transformative event (Tisdell, 2012) or when the participant is much older and more capable of subjective reframing (Merriam, 2004). Most importantly, however, Kylie's transformation toward social action void of a stated or observed change in socio-political epistemology aligns with Johnson-Bailey and Alfred's (2006) observation that marginalization may be the most powerful catalyst for transformational learning (see also Johnson-Bailey, 2012).

What is each student's racial, economic, gender, and cultural identity? How does each student's identity impact their worldview and their transformations (or lack thereof) during travel. Twenty-year-old Kylie was the participant with whom I had the most difficult time understanding her socio-economic identity. She selfidentified as lower-middle class during the member-check interview, and provided her 
need for work-study and Pell grants to pay for college as evidence. Kylie also emphasized that she paid for both college and study-abroad herself from an insurance settlement from a childhood accident and from the $\$ 200$ a year her grandparents gave her for Christmas. Unlike several of the upper-middle class students, Kylie worked throughout high school and summer breaks of college to pay for college expenses herself. Kylie's mom was a director of a pre-school and had the same job for Kylie's whole life. However, Kylie's dad experienced a series of layoffs after the tech crash of 2008. This unemployment period coincided with middle and high school for Kylie. Previous to 2008, her dad worked in informational technology for a large telecommunication company. When the economy crashed and the technology bubble burst, Kylie's dad attempted sales for a large trucking company. However, a commission-based sales job did not fare well in the Great Recession. Therefore, Kylie explained that her dad went from making solid upper-middle class income pre-2008 to spending the family's entire savings going back to school as a fifty-year-old. Kylie stated in the member-check interview that she hated the assumption, "You live in a mansion; You're rich," that attended growing up in her wealthy suburb. Kylie maintained that she "awkwardly" lived in "just a split-level" that was down the street from "mansions."

Kylie's family's fall from upper-middle class to lower-middle class (from Kylie's self-identification) aligns with Smiley and West's (2012) label of the "New Poor" that came about as a result of the Great Recession. Smiley and West argue that a whole generation of White Americans experienced poverty (or in Kylie's case reduced income) for the first time as a result of the Great Recession. Nevertheless, according Smiley and 
West, the "New Poor" status is socially complicated with generations of White privilege and economic supports that are typically absent from individuals living in multigenerational poverty. For example, although Kylie attended school on Pell grants, her mom gave her a job every summer and her grandparents gave her $\$ 200$ every Christmas to help pay for college. Furthermore, Kylie paid for college (and the studyabroad program) from a trust fund that was created from an insurance settlement from a childhood accident. Consequently, Kylie's sense of under-privileged status was informed through the lens of economic supports that are often unavailable to economically repressed individuals through institutional class and race oppression.

Notwithstanding the negative financial impact to Kylie's family from the Great Recession, she was raised in an environment where peers, family, and classmates experienced considerable class privilege. As mentioned in Kylie's introduction, she was from the same wealthy suburb where Kristene and Anne resided (which was in the richest county in their Midwestern state). Narratives of this class privilege observably impacted Kylie's comments and worldview. When I asked her why she desired to attend the studyabroad program, Kylie answered, "Seeing a bunch of friends from other universities that had gone to, like, Spain or other places abroad, I was kind of jealous." Furthermore, Kylie was excited that her "mom thought I needed a whole new wardrobe to come to Spain, so, I couldn't really complain." In addition, Kylie stated that she felt the major theme of her trip to Europe was a "rite-of-passage" where she could experience "freedom," a "care-to-the-wind" and do "whatever we wanted." Kylie consistently 
referred to the study-abroad program as a "vacation" and stated that it "seemed like a dream" and "didn’t seem like real life."

Kylie additionally had a view of "others" that CRT scholars argue reinforce White supremacy through essentializing and exotifying racially and economically disadvantaged individuals (Bell, 2009; Delgado, 2009b; Tatum, 2009). Kylie explained that although she was "lucky" to have a mom that could be around to raise her and her siblings, she was "jealous" of her poor peers "“cause they [got] to hang out with their teachers, and ... their friends all day long" instead of their parents after school and in the summers. She felt the study-abroad program gave her the "freedom" to "have... that dysfunctional childhood you're always kind of jealous of." Moreover, Kylie was also prone to use “othering" language, calling citizens of Spain "natives" and was excited to be living an "urban" lifestyle in an apartment.

Smiley and West (2012) assert that the socio-economic hardships faced by the "New Poor" as a result of the 2008 Great Recession could be a catalyst for seeking out allies for social justice. Like Johnson-Bailey and Alfred (2006), they maintain that marginalization, through even short-term financial setbacks created a window for White, upper-class individuals to experience what economically and racially underserved individuals have faced for generations. Although Kylie's mental framework of being "nice" to English learners in the United States is infused with a framework of class and racial privilege, I believe that Smiley and West's vision of creating long-term allies for social justice from short-term marginalization may come out of Kylie's experiences of feeling "othered" in Spain. Like Tisdell (2012) maintains, epochal transformation that 
requires a complete epistemic reframing often takes a series of transformative events rather than a single experience like the study-abroad program.

Participant Seven—“Kynzee"—Willingness as Inter-cultural Permeability toward Socio-Cultural Subjective Reframing.

An observation I made during Kynzee's presentation in Juan's class during the first week of the study-abroad program highlights Kynzee's disposition and must precede any other information about her experiences. I wrote in my field notes:

I cannot overstate how fun and exciting Kynzee is. She really struggles with Spanish, yet she is fearless. I love her attitude.... Juan smiles so big while she presents and keeps jumping in to help her present. I hope that this experience will be both a linguistic and socio-culturally [transformative experience] for her. She ... oozes the willingness that Mezirow and other TLT theorists describe [that is a pre-requisite to transformative learning].

Repeatedly over the first couple of weeks I noted Kynzee's "outgoing," "participative," "enthusiastic," and "willing" nature. During the Alhambra cultural excursion, I wrote that Kynzee and Tim were "embodiments of Mezirow's willingness that is necessary for transformative learning to take place. Ironically, they are also the two that have the most difficult time with Spanish.”

There was a difference between Kynzee and Tim's willingness, however. Tim's "people are people" version of willingness negated the possible transformative experiences that arise from being epistemologically permeated by an "other" culture. In addition, Tim was more of an appreciative observer of culture (a mode of taking). In 
contrast, Kynzee was a self-proclaimed "people-pleaser" who valued interpersonal relationships over all else. Consequently, she valued cultural immersion and dialogue (a mode of cultural symbiosis and interchange).

According to Kynzee the "purpose" of the study-abroad program was to "learn Spanish.” She said, "I'm a Spanish major, so it's always been a dream of mine.... If you're going to learn Spanish, the place to go is Spain!" However, Kynzee was additionally on an "adventure" to find her heritage. Kynzee self-identified as "HispanicAmerican," "Hispanic," "half-Mexican," and "Latina" throughout the study-abroad program. She explained her racial identity in the entrance interview, "I want to do [the study-abroad program] because I want to learn about the culture. My family is Hispanic.... My grandfather's from Mexico.” Moreover, Kynzee commented on the complexity of identifying as Latina, but not being a heritage Spanish speaker or being socio-racially constructed as Latina. She said, “[I'm] blue-eyed, blonde hair, [have] light-skin, but if you saw the rest of my family ... they're extremely dark. Like, they look like authentic Mexicans. And then there's me." Learning Spanish was a way for Kynzee to connect to the rich "cultural heritage" of her grandfather. She explained in the entrance interview:

I definitely identify with my heritage, and I think that's probably why I'm learning Spanish, 'cause I just want to get closer to that.... I didn't know my grandfather.... My grandmother did not like him speaking Spanish to my mom and my uncles, and so, they never knew Spanish.... So, it's definitely something that I'm using to connect back to that. 
Kynzee became extremely excited when I told her that her last name came from the south of Spain as we were on a bus headed toward Granada (situated in the south of Spain).

Kynzee lived with her mom, her maternal uncle (who "in all sense of the word [was her] dad"), and her two brothers, who with Kynzee, were triplets. Kynzee never specifically stated her socio-economic class, and something about her optimistic nature made me feel that directly asking her would result in an answer that she "had it better than many." Nevertheless, Kynzee was from a small town about 30 miles away from PSU that had 4,000 residents, of whom $95 \%$ of the population was White, with a median household income of $\$ 29,000$ (Wikipedia.org). Kynzee participated in college through grants, scholarships, work-study, and other programs meant to beneficial to poor and working-class families. Obtaining sufficient money for the study-abroad program was a "miracle" for Kynzee that was "very abrupt ... unexpected, and it was quite a blessing to my family." She explained in the entrance interview:

I had the blessing of my funds for this trip, and as soon as I was told that, that we had some extra ... funding ... in my family ... I was like, “Mom! It's a sign. It's karma. It's a miracle. This is what God wanted me to do! He wants me to go to Spain!”

There was always a sense from Kynzee's descriptions that her family worked communally to provide for each other whenever she discussed money. However, this communal nature should not be confused with a lack of opportunities or with poverty. In fact, Kynzee had the most travel experience of any of the participants in the study-abroad program. Kynzee's uncle traveled frequently for business, so he would take Kynzee and 
her brothers whenever he had extra travel miles vouchers. Kynzee had been to most of the regions of the United States as well as a visit to Mexico for a few days with her uncle to learn Spanish. Additionally, Kynzee had been on a two-week study-abroad program to Costa Rica with an emphasis in social work. In the study-abroad program, Kynzee stayed with a host family similar to the LU study-abroad program.

To what extent do seven students experience the elements of transformative learning during a month-long study-abroad experience in Spain? Similar to previous participants, I will address cultural shocks, adjustments, and surprises Kynzee underwent. Next, I will discuss Kynzee's unique attitude of culturally inclusive willingness and address how this openness created an environment where socio-political subjective reframing could happen. Lastly, I will describe an interview with her host brother that led to Kynzee's biggest socio-political transformation - a critique of America's gun culture.

Culture shocks. Kynzee never experienced cultural shock that would qualify as a cognitive dissonance under Mezirow's (2000) model of TLT. Nevertheless, she did experience a disappointment, a cultural adjustment, and several cultural surprises.

Cultural disappointment: Drugs. Kynzee was disappointed that Tess's (discussed later) boyfriend, Paco, used drugs. She recounted what happened during the festivals in the exit interview:

[Paco] tells me to hold something for him, and it was a cigarette, and I was like... "Okay I'll hold the cigarette." And then the next thing I know he's putting things into my hand, which I do not know what they are. And there's a little green cube. 
And I'm like, “Drugs! Drugs! Drugs!” I push 'em back at him and I'm like, “I don't want it...." So, it just kinda started killing my vibe. And me and Becky (sic) were like, "This is getting a little dangerous."

From Kynzee's description above, I assume the drug was hashish. However, in contrast to Kylie's essentialization of thinking her host "mom" represented "how the people really are" in Spain, Kynzee was solely disappointed in Paco. She also was concerned that her "reputation" would be tarnished with her family, her host family, and the clubs she represented back at PSU if she were associated with drugs in any way. Therefore, Kynzee and Becky walked home instead of continuing to celebrate the festivals with Paco. Cultural adjustment/surprise: Affection. In the entrance interview, I asked Kynzee what culture shocks she had experienced. She did not seem "shocked" by anything. I had to follow up by asking if there was anything that caught her off guard. She responded that it was "surprising" to see how affection people were in Spain. Kynzee explained:

The first thing I noticed was ... how affectionate people are.... There was a couple sitting in front of me [at the airport], and they just started making out, 'cause I guess ... somebody was leaving. So, you know, they had to make sure their feelings were known. [Chuckles]. And so then ... other couples did that ... and not just young couples. We're talking like ... aged, married couples.... I think that's awesome. I'm a romantic at heart, so I think ... that's cute.... It's a custom I wish that could be brought back to the U.S. 
Additionally, Kynzee and Tess (whose dad is from Spain) were the first participants to appear comfortable giving besitos. During the member check interview, I followed up about affection. Kynzee responded that she missed the affectionate nature of the people of Spain and has tried to carry the practice of besitos back to the United States:

Now, if I see somebody of a Hispanic descent I do like to go in for the little kiss on the cheek and I just hope and pray that that's okay with them. I guess that's about it. I miss Spain a lot. I want to go back. I feel so bad because I felt like I left my home, which is not true. I came home.

Cultural surprise: Food. Kynzee indicated in the entrance interview that one of her favorite things about visiting a culture is to experience their food. In her blog, Kynzee wrote that the food is "almost like a representation of the country itself. With all the different flavors and textures, there is always something new to discover and enjoy." When Kynzee overheard Kylie inviting me to look at her blog, Kynzee invited me to follow hers as well. Although Kynzee's blog only had four entries, a large part of it was dedicated to the food of Spain. It appeared that even though she was anticipating enjoying the food, her host “mom" outperformed Kynzee's expectations. When I followed up in the member-check interview, Kynzee explained, "Food ... [is] a crosscultural thing ... that ... brings people together whether you're in the United States and whether you speak the same language or not."

Cultural surprise: Nocturnal, callejero nature of Levantinos. Several times throughout the study-abroad program, outgoing Kynzee commented on how much she loved that many people spent much of their time outdoors. In addition, she spoke highly 
in the LU classes of how much she enjoyed discovering a city by walking around and taking public transport. In the entrance interview she said, "And I like how much people walk here. I like that $a$ lot $\ldots$ and how everybody takes the tram.” Furthermore, Kynzee stated that the nocturnal, callejero nature of the people of Levante took her by surprise, but in follow-up interviews she told me that she came to enjoy the walking around in the evenings. In her blog, Kynzee stated, "At first I thought it was pretty late to be walking around the streets at night but that is what is so cool about the Spanish lifestyle, they spend most of their leisure time during the late night and early morning hours."

Cultural surprise: Landscape. Kynzee said she was amazed to see how close the mountains were to the beach, which was a sharp contrast to the plains and rolling hills of the Midwestern United States. In the entrance interview, Kynzee explained:

I really like ... that I can go to the beach, and see ... a mountain from the beach! So that's really awesome, 'cause, when I was younger, I lived in Tennessee, and so I grew up ... in the Appalachian [mispronounced] -whatever mountain range.... So, seeing mountains always has ... a sentimental ... memory for me. Wake up to the morning ... to the fresh mountain air, and beach air ... that's quite a combination. It's really nice. I really enjoy it. I really like the scenery. Kynzee also said in the entrance interview, “I don't think I believed Dr. Green when he told me I was going to live five minutes from the beach, and I do. So that's awesome." Cultural surprise: Bullfight. In contrast to many of the participants, Kynzee appeared to sincerely enjoy the bullfight. I asked her about what she was experiencing and she told me that she was "in love with the experience" and that the LU professors and 
Dr. Green "adequately prepared" her for it. Like Becky, Kynzee's "mom” dressed her in a traditional dress, put her hair in an updo, and put flowers in her hair. In the exit interview, I asked Kynzee about the bullfight. She responded:

When I was growing up I didn't know they ... killed the bull; I just thought they ... played around with it. But as I got older ... that's something you kind of pick up on, and we had plenty ...[of preparation].... All of our teachers said something, you said something ... my host-mommy ... said that there's going to be six bulls, and they will all die. So, mentally, I was prepared.... I [went] into it knowing that it's a cultural thing.... You have to look at it from their perspective, you know? It's about being open-minded, and I think part of the reason why maybe some ... have an issue with it is 'cause ... they hold on to ... their ethnocentrism.... Being from the United States ... we don't do that. And that takes away from the experience, and you go home saying, "Ugh, this was horrible!" instead of, "Oh! What an interesting thing I got to see and appreciate...." So, I just try to keep an open perspective, especially studying abroad.

Willingness (as openness, humanity, and permeability). Kynzee appeared to genuinely enjoy people. In her blog, Kynzee described an experience that occurred on the plane ride to Madrid. She offered to play cards with a woman from the Middle East who did not speak English. Kynzee gestured how to play, after which they played for several hours, laughing and sharing food with each other. When the plane landed, 
Kynzee helped the woman out of her chair and walked her to find a wheelchair. Before Kynzee could leave, the woman hugged and kissed her repeatedly.

This authentic love for humanity was a trademark that Juan, Eva, and Dr. Green all discussed that impressed them about Kynzee. As said above, there was nothing observably voyeuristic or appropriative in Kynzee's nature. Rather, Kynzee appeared to emanate the humanist axiology of TLT (Brookfield, 2012; Pritchard, Morgan, \& Ateljevic, 2011). Furthermore, Kynzee was not only interested in the individuals, but was also fascinated with their cultures and what interested them. She wanted to go to every festival, restaurant, ice cream parlor, activity that was available to the students. For example, during the festivals Dr. Green announced that the LU had made special arrangements to attend the fireworks from a special paid booth adjacent to where the fireworks went off. Dr. Green asked if any of the students were interested. None of them beside Kynzee raised their hands. Kynzee reprimanded her classmates in English, "You've gotta be kidding me. The university went out of their way to provide this activity for free. Who's going with me?" She convinced a couple of her classmates to go. Ironically, out of all of the participants, Kynzee had the least need to go to something like this. Her host family's flat was above where all of the fireworks went off. She could see them simply by walking out to her balcony. It appeared that she genuinely wanted others to be able to enjoy what she did daily from her flat. Furthermore, she seemed to express genuine concern for the time of the events coordinator, a man she had only seen twice before. 
Kynzee's permeable nature extended to the LU classes. Multiple times throughout the month, Kynzee asked questions that spurred questions in both professor's classes. On the day the king abdicated his throne, Kynzee asked Juan what all of the red, yellow, and purple flags were hanging from their windows. I wrote in my field notes, "You can tell that Juan wasn't expecting to talk about this, but since it is such a great question and a current event, he runs with it." Juan spent the next thirty minutes discussing the different political factions that were being discussed in the news (the flags represented individuals clamoring for a republic to replace the constitutional monarchy of Spain). I asked Kynzee about this interchange in the entrance interview and she replied: We're living in a historic moment in Spain right now, with the ... changing of the ... king to the prince to be king.... And to be able to live here and to experience that with them, is awesome. I love that ... because ... [in] my generation, we've experienced a lot in the United States, too, with 9-11, and our first Black president. Kynzee's openness to political discussion was different from Jennifer's interest in politics as a political science major. In contrast to Jennifer, Kynzee appeared to value the professors' opinions and asked questions to clarify consistently throughout the classes. No topic was off limits: stereotypes, racism, socialism, political correctness, religion, and feminism were all topics that Kynzee asked the professors to explain from a Spanish perspective. After Kynzee's final presentation, I wrote the following paragraph in my field notes:

I really believe that [Kynzee] gained more in a more permeable/open-minded 
worldview than [she has] in Spanish.... I was intrigued at [her] capacity to debate politics, religiosity, and other complicated subjects during ... presentations [in] Juan's class. She went beyond the Left vs. Right [ false binaries] we have in the United States.... It was not the depth of the argument that impressed me, but [her] willingness to consider new ideas.... [Kynzee] came up with ideas that you could tell came from actually thinking about it rather than regurgitating ideas [she] had seen on news shows.

This willingness led to subjective reframing about the United States gun culture, and her place in it.

Transformations. One of the topics that Juan discussed regularly in class was stereotypes and the negative impact they can have. From their comments in class, it was evident that many of the students had never considered that there might be negative stereotypes about the United States. Consequently, Juan gave the students an assignment to interview a European about stereotypes regarding the United States. Kynzee decided to interview her host "brother," who was a travel blogger who had been to many parts of the world. She was shocked at her host "brother's" critique of United States gun culture. Kynzee explained that her host brother replied, "Yeah. You guys have guns and you guys have ... all these shootings. You know, you can't walk down your street, without worrying about being safe." Kynzee discussed her reactions to this thought in the exit interview:

And I was like, “Oh my gosh! I didn't even think about that!” I was like, “I’ve been here a month and I haven't heard about any shootings. We hear about a 
TRAVEL FOR TRANSFORMATION

shooting every other week in the United States, you know?" And I was like, "Wow, he's so right!" And that's why I don't really take an absolute political stance.... 'Cause I try to keep ... [an] open-mind, but I do think there needs to be some enforcement in the gun laws in the United States.... Obviously, Spain can do it. These people are out; they have their children out until like 3:00 or 4:00 in the morning.... You can't even be out past dark in the United States.... Wow! That was really surprising. I mean, it was eye-opening. So, that's one thing I kinda keep coming back to, is how safe I feel in the city ... I mean, this city's what? It's huge! [Levante had about 350,000 inhabitants during June, up to 500,000 during the festivals, according to Juan]. And I can't walk to class, to and from, at night, [in the town where PSU is located], which is $20,000 \ldots$. My mom's always warning me, “Kynzee, I don't want you walking out by yourself at night.... Carry your pepper spray...." And it's so true, because at the beginning of this school year, we had all those shootings and stabbings in bar parking lots [near PSU]. And here, kids are out 'til 2:00 and 3:00 in the morning.... So that is kind of a difference that I would ... like to see change in the United States, compared to Spain.

Kynzee went on to explain that a "lightbulb hit" her as her host "brother" spoke about violence in the United States. She maintained that she never would have "come to this conclusion about how violent Americans are [if] someone hadn't told [her]." Kynzee experienced a disorienting dilemma (Mezirow, 2000) wherein she could no longer maintain the viewpoint she previously held about the United States being safe. Kynzee 
experienced what Tisdell (2012) called an epochal transformation — a complete, dramatic reordering of thought about society and her place in it (see also O'Sullivan, 1999, 2012). Kynzee further explained:

I always knew people say stuff about how American movies are violent and how European movies are more sensual and I understood that but it didn't actually hit me that our culture is actually a reflection of those movies. [Rather], I should say that the movies reflect our culture, so that's always something that has stuck with me especially since I live with it.... [PSU] campus actually doesn't have any current policies and we're actually being forced to implement concealed carry [of firearms], so it's something that's always kind of stuck in the back of my mind about how Americans are so desensitized to guns, and violence, and killing, ... and death of other human beings.

In the member-check interview, Kynzee informed me that she misses the feeling of feeling "safe" in Spain because she does not "feel the same way in the United States," despite being "a very independent woman who can take care of herself." She added that her uncle noticed the difference in feeling when he visited her in Spain, as well. Because Kynzee continued to discuss gun culture at home, I am confident that she experienced the dramatic shift in consciousness representative of socio-cultural transformation (Mezirow, 2012; O’Sullivan, 2012). Furthermore, from the way she discussed political events going on impacting the university she attended, I believe she experienced an irreversible, epochal change that both provided her with a new way of seeing the world and a more autonomous way to act in it (Taylor \& Elias, 2012; Tisdell, 2012). 
What role did encounters with cultural and linguistic "otherness" play in the transformative learning (or lack thereof) of each participant? In this section, I will discuss Kynzee's sense of radical cultural inclusion and symbiotic cultural interchange with her host family. Subsequently, I will explain how this sense of inclusion was not only with her host family, but with professors at LU (and others), as well. Lastly, I will provide a description of Kynzee's difficulty with Spanish and analyze the possibility for Kynzee to legitimately experience linguistic "otherness" from the lens of her limited Spanish proficiency.

Extreme cultural inclusion: Host family. One thing Kynzee said in the entrance interview summed up Kynzee's experiences with her host "mom.” Kynzee said, "That evening I met my host "Mom," Mami and I knew as soon as I met her, we were going to get along great." As mentioned in Becky's findings, Kynzee's host “mom” and Becky's host "mom" were dear friends. Both of them were empty nesters (although Kynzee's host "brother" would return home in between professional travel assignments as a travel blogger). Their host "moms" were upper-middle class women married to retired professionals and enjoyed hosting students, hoping to highlight the positives of Spanish culture. Kynzee called her host "mom," Mami (placing the accent on the second syllable instead of the first as is correct in Spanish, [ma-mí]). Because Kynzee used the term Mamí, I will write it the way she pronounced it, rather than the way it is correctly written and pronounced in Spanish.

According to Kynzee's descriptions, Kynzee's and Becky’s host “moms” really "went out of their way" to help them "experience the culture." For example, in the first 
weekend of the study-abroad program (before classes even started), Mami took Kynzee and Becky (along with her host "mom") on a tour of the city. They had:

A wonderful time seeing the city and enjoying the ambiance of such beautiful buildings at night. As we were walking down the crowded streets of the Spanish youth, our Mamis treated us to some homemade ice cream at a local ice cream parlor.

The two families often joined to eat out, play board games, and take evening strolls along the beach. During the festivals, the two families saw fireworks, went shopping, went out to bars, went dancing, got dressed up in traditional Levantino attire, watched parades, hosted a dinner party, went to neighborhood block parties, and saw a comedian. Furthermore, Kynzee's family lived adjacent to the main plaza of the city where daily fireworks and other events happened. Accordingly, Mami often invited the whole group of study-abroad students to celebrate the festivals at her house. I asked Kynzee in the exit interview, "Your 'Mom' is a party animal, isn't she?" Kynzee replied:

She's fun! That's for sure.... She doesn't really drink, either [Kynzee rarely drank alcohol]. I've noticed that $\ldots$ because ... she'll serve beer to all her friends for lunch, but she'll have a glass of like orange juice or something. So ... I think she loves being a host—a host-anything.

Throughout the study-abroad program, Kynzee made statements like, "I have the best Mami ever!" and, "She is the bomb!" 
This closeness with Mamí also enriched Kynzee's relationship with Becky. They knew each other before the study-abroad program. However, as a result of all of the fun things they did with their host "moms," Kynzee stated in the exit interview:

Because my Mami's best friends with Becky's Mami ... me and Becky have become really [good] friends.... I love how much alike we are. And I feel like we're ... thick as thieves now. And so, I'm glad that our Mamis are best friends, 'cause we hang out all the time.

In the entrance interview, Kynzee explained that she felt that her previous study abroad in Costa Rica helped prepare her for living with a host family, "I don't want to talk about Costa Rica a lot, but...it has definitely helped me prepare for this trip, because, I knew [how to] live with a host-family." Furthermore, another trend I observed as early as the first couple of days, Kynzee started using affectionate indicators like "my Mami" to describe her host "mom."

Perhaps the most important characteristic of Kynzee's cultural and linguistic immersion was a sense of respect for her host family and their culture. In the entrance interview Kynzee explained:

[Mamí's] had ... a ton of girls as host-daughters for several years, and so she has all their pictures up. What's really nice is I get my own room, and I have my own bathroom, and ... She's an awesome "mom." She ... really takes care of me.... Every morning — and it doesn't bother me—-she'll wake me up ... and ... when I come home from wherever I've been ... my room will be clean. [In return], I try to be a cleanly person, especially since I'm in somebody else's house. 
In contrast to other participants who often shared service-oriented narratives of their host families, Kynzee valued and tried to reciprocate the kindness of Mami. Within the first week, I observed Kynzee and Becky shared positive stories and had a different sense about being a guest in another person's house. In contrast to Anne, Jennifer, and Kylie, Kynzee's vision of herself was displayed in her optimistic attitude about her host family, while the other participants often discussed and compared the negative aspects of their host families when the students gathered together. Examples of negative narratives among the other participants included room size, food provided, extra-curricular family activities, and family norms and customs. This negativity among the other participants deeply troubled Kynzee. She expressed her annoyance in the exit interview:

I feel bad for some of these girls 'cause their "Mamis are mean." Granted, I think some of these girls have put it on themselves ... which makes me really mad. And then, that's the other thing that's bothered me that I probably haven't talked about yet, is how some of these girls are just...wah-babies. Fuddy-duddies, is what I like to say.... I just wish they were more respectful.... I feel especially since you're not from here, [respect] needs to be top priority.... And what really bothers me is that they whine and complain about how we have homework. I was like, "What'd you think you were coming here for? Vacation?" In contrast, Kynzee's frame of reference as a houseguest formed a symbiotic relationship wherein both the host family and Kynzee were enriched (this was true with Becky, as well). Like Kristene, I often observed Kynzee shopping for little trinkets to show gratitude to her host family. This ontological stance of herself as a grateful house 
guest, rather than someone paying for a room and service often came through as a positive, rather than a deficit view of her host family. For example, Kynzee loved the location of her flat:

I'm right in [central plaza of the city]. Like, you have this statue ... if I go out ... [to] my balcony, and if I just look out my window, like right there is the plaza. I'm literally, like, a one-minute walk to the tram station.... It's really conveniently located.

(For a contrasting narrative, Jennifer lived just a few blocks away and often complained that her house was not near anything). Kynzee also expressed sincere concern for the health and happiness of her host family. In exchange, it seemed like Mami reciprocated this affection, sharing with Kynzee intimate family details like knowledge of her breast cancer, who her host "brother" was dating, and major surgeries her host "father" needed. In addition, Kynzee told me during the third week that Mami was always trying to "fix [her] up with Spanish boys." I wrote in my field notes that I thought Mami might have been trying to introduce Kynzee to other Spanish college students, however, to further immerse Kynzee in the culture she loved.

As a result of an empathetic, respectful worldview, Kynzee became an intercultural experiencer and dialoguer rather than a tourist who took from the culture. Kynzee expressed this contrast from many of the other participants in the exit interview: I think by far me and Becky had ... it ... very different ... because [of our] comfortability with people.... That's ... how I knew so fast with Mami that she was going to be a [great] host mom and I [would] do my best to be a great host 
daughter.... I think part of it was that most people did not come in with that mentality. They came in with a mentality of "You're here to help me," not the other way around. Whereas, [my narrative is] "I am here to learn from you." I think with our host Mamis being so open ... [we] embraced [them], they embraced us learning about their culture, and we reciprocated that, where I don't think some of the other students did.

Additionally, Kynzee discussed the positives of having a respectful attitude toward the host family in the member-check interview, "My appreciation for other cultures [and other people] has strengthened.... I know if I didn't have my Mami', this experience could have been horrible." My observations (filtered through the statements of Kynzee's and Becky's host parents) led me to believe that the participants' positivity/negativity was a greater influence on the intercultural exchange than the host parents' desire to provide a positive experience. However, one outlier to this grounded theory would be Kylie's host "mom," who was extremely rude (from Kylie's stories), which seemed to form an exponentially negative interchange loop between Kylie and her host "mom." Nevertheless, Kynzee clarified the idea of inter-cultural respect and dialogue in the member-check interview:

Part of it is just simple manners. If I were going to go spend the night at my friends house here in the United States I would act the same way.... I'm not paying [my host family] to be my servant.... I'm paying them to teach me how to live in another culture and the Spanish society.... To think that just because I [can afford] to live in your home does not mean that I should be treated as if I'm 
better or more.... I think part of it is just simple human kindness and respect.... My Mami didn't have to do all these things that she did for me, [but] ... her generosity and my willingness to accept it- - I think is what made the big difference in the end. And I still talk to her; It's so great. And [my host "brother"] just "friended" me on Facebook.

When I asked her what the "theme of her trip" was in the member-check interview, Kynzee replied:

Just appreciate the opportunity and take advantage [of what] you have been given. That's what I think I noticed how some people didn't use their time to the most, [and] how I think they didn't use the time the way that I did, and for me studying abroad as an experience and that's what you are there to do. So, when you ... waste your time ... sleeping and talking to people [in the United States]... I think you're losing opportunities that you could have taken. So, that was a big thing for me as I wanted to see, to do, to experience as much as I could while I was there, because I knew that this was a gift from God that I was able to go to Spain.

Cultural inclusion: Professors and everyone. Everyone with whom I saw Kynzee interact during the study-abroad program had positive things to say about her. For example, as mentioned earlier, Juan smiled every time she presented or entered a room. Even though Kynzee had arguably the lowest proficiency in Spanish, both Juan and Ana helped her get through her presentations in the LU classes. Kynzee made up for her Spanish deficit by preparing extremely fun, energetic presentations, which always 
included some game show format. I wrote in my field notes during Kynzee's last presentation in Juan's class:

Kynzee is so prepared. Juan cannot help smiling every time Kynzee talks. She struggles with Spanish about as much as Tim, but she comes with a game activity, she has a great PowerPoint, and her enthusiasm is both contagious and extremely "Spanish." Juan consistently helps her out throughout the presentation with vocabulary and verb conjugations.

Moreover, as mentioned in the introduction, Kynzee often asked questions which showed that she was interested in the topics presented in both classes. From an educator's viewpoint, I observed multiple occasions when Kynzee's questions created discussions that were not part of the lesson plan (observable by the departure from the professors' prepared PowerPoints [Juan's class] or textbooks [Ana's class]). Furthermore, any time Dr. Green mentioned Kynzee he smiled and expressed his admiration for her discipline in speaking Spanish outside of class (in contrast to the other participants). He told me he wished all participants would seek an immersive experience in the way Kynzee did. Lastly, Kynzee adapted the Spanish custom of walking into a room, loudly greeting everyone with, "¡Hola!" and then individually greeting each person in the room with besitos. After about two weeks, I noted to Juan that Kynzee had adapted to the Spanish custom of entering a room. He replied, "Indeed, she has," and then he used Kynzee's entrance as an example to the other participants of what was culturally expected when a person enters a room in Spain. 
It was not only individuals in positions of power with whom Kynzee formed positive relationships, however. As mentioned during Kristene's and Jennifer's findings, the "extremely handsome" tour guide to Granada, Manolo, was a similar age to the participants in the study-abroad program. After we went to a flamenco show, the group returned to the hotel at about 10:00 p.m. Manolo explained to the students that it was culturally common — even expected — for Spanish youth to stay out until at least 2:00 or 3:00 in the morning on a Friday evening. He said, "Even my grandmother stays up until midnight." He invited the participants out for drinks and dancing (in a very nonthreatening way). Only Kynzee and Tess (discussed later) took him up on his offer. As stated in Tim's findings, this research has led me to question Mezirow's (2000) use of the word "willingness." Both Tim and Kynzee embodied a willing attitude, yet only Kynzee experienced socio-cultural transformation. While research involving eight participants is not generalizeable (Marshall \& Rossman, 2011), the purpose of constructivist grounded theory is to systematically generate new ideas for further study, from the viewpoints of the participants (Charmaz, 2003). Importantly, Kynzee displayed a different attitude about the host culture that was different than Tim's "people are people" narrative that I likened to the myth of colorblindness discussed in CRT scholarship (Gillborn, 2009; Leonardo, 2009).

In contrast to Tim, Kynzee displayed a respect for the values and funds of knowledge of the host culture (Ross, 2010); was willing to critically reflect upon the knowledge gained from an "other" culture (Brookfield, 2012); was willing to be immersed in —and permeated by—the host culture (Brown, 2009; Pritchard, Morgan, \& 
Ateljevic, 2011); and was willing to be seen as a member of the host culture (Foster, 1997). Therefore, mutual permeability and respect led to intimate intercultural experiences and in-depth discussions about Kynzee's own culture (Morgan, 2010; Ross, 2010). Consequently, Kynzee opened herself to the opportunity to develop critical selfconsciousness about United States gun culture and desire to act to improve society (Cordero \& Rodriguez, 2009). Indeed, Kynzee's subjective reframing led her to "examine, question and expand [her] cultural assumptions, acquiring behavioral and cognitive repertoires that foster critical consciousness" (Cordero \& Rodriguez, 2009, p. 136).

Cultural inclusion: Limited Spanish proficiency. Kynzee's "fearless" (my field notes) attitude about attempting to speak Spanish combined with her "outgoing," "energetic" nature, created an atmosphere where she developed rapport and trust with members of the host culture, despite her limited proficiency. I observed she attempted to speak Spanish even when other students would not. Kynzee often recounted funny anecdotes about her failures in attempting to speak, despite her limited proficiency. One story perfectly illustrates the combination of Kynzee's fearlessness with the language, the often-comical outcomes, and yet, still shows how member of the host culture valued her effort.

One day I suffered some mild heat exhaustion and woke up the next morning with a terrible migraine headache. I went to Juan's class to observe the students, but felt nauseated, was dizzy, was seeing lights that did not exist, and had a pounding headache. In between classes, Kynzee asked me what was wrong. I explained and said I needed to 
go home. I taught her the Spanish word for migraine, jaqueca (more commonly used in Spain), but it was not sticking with Kynzee. Next, I taught her the cognate migraña (less commonly used, but correct), which she could remember. I asked Kynzee if she would offer my apologies to Ana and explain why I went home. I had her repeat migraña four or five times and went home to sleep off the migraine. When I returned the following day, I apologized to Ana for going home due to a migraña, and Ana started to laugh hysterically. She told me that Kynzee approached her the previous day and said I needed to go home because I had a really, really big mierda (shit).

Fascinatingly, Kynzee's efforts to speak Spanish, even when she did not have to, endeared her to members of the host culture, rather than made her "othered" by them. This vulnerability not only opened her to a state of permeability, it appeared to open members of the host community to want to share their knowledge with her, as well (Goulah, 2007). Kynzee's willingness to be seen as a representative member of the host culture (Ross, 2010) overcame the linguistic "otherness" that often is experienced by study-abroad students who are immersed in a culture whose language is not their heritage language (Foster, 1997).

What is each student's racial, economic, gender, and cultural identity? How does each student's identity impact their worldview and their transformations (or lack thereof) during travel? As stated in Kynzee's introduction, she had traveled more than any of the participants, having visited most regions of the United States, Mexico, and Costa Rica prior to the study-abroad program. She also moved around the Appalachian Mountains and the Midwest as a child before settling in her town near PSU. 
Furthermore, even though Kynzee never specifically mentioned socio-economic class, a review of data from her interviews and my observations manifested a lower-middle or working class background. Also, as mentioned in the introduction, Kynzee identified as Latina.

Race as a social construct. Kynzee discussed during the member-check interview her cultural identity as a Latina, complicated by the way society and her family constructed her identity:

Even though I self identify [as Latina], it does not come across to anyone else. If anyone were to see me or to listen to me [speak Spanish] they would obviously know, "She's a gringa." And, I accept that my family's not super into our Hispanic heritage, either. The only thing that I'm holding onto is that my grandfather is from Mexico and I don't know him. He passed away when I was younger and he never taught Spanish [to] anybody in my family. And, so we do have roots there, but it's not something ... that's very predominant in my family culture.... We are "strong" Americans, you could say. But that being said, I do like to think by ... learning Spanish I reach back to those roots that I haven't [experienced in] my family.... [My] last name [common Spanish last name] ... [further] motivates me to be part of that culture.... My mom thinks I disown American culture, which is by no means true. I consider myself a hard-core, patriotic, all-loving American, but I do want to get back to the Hispanic roots that my family does come from, and so that's the mentality that I have. I want to learn as much as I can. 
Kynzee's worldview aligns with the CRT concept that race is a social, not biological construct (Delgado \& Stefancic, 2001; Parker \& Lynn, 2009; Solórzano \& Yosso, 2009). Kynzee was aware of how she was constructed by society, and felt the pressures of her family to identify with White, North American ideology. Nevertheless, Kynzee actively worked against her privilege as a socially and family-constructed White individual in search of her Hispanic cultural heritage. Rather than obfuscate and whitewash her Latina identity, Kynzee wanted to celebrate and understand her "roots," even though it was against her financial and social self-interest to do so (Tatum, 2009). Moreover, Kynzee rejected the "racial amnesia" (Leonardo, 2009, p. 268) that her family practiced through assimilationist frames of reference void of the racial and cultural intersections that brought her family to that place in society (Crenshaw, 2009). In addition, in contrast to Brookfield's (2012) observation that it is difficult for individuals occupying privileged positions to be aware of their privilege, Kynzee was self-reflective and acknowledged the unearned benefits that she received because society constructed her as White. Consequently, I felt no (mis)appropriation of her grandfather's heritage. Rather, Kynzee expressed self-awareness and eloquent expression of the complex intersections between her socially constructed Whiteness and her deep connection to her grandfather's roots.

Impacts of race and class on Kynzee's socio-cultural transformation. From the data collected and analyzed for this research, it is impossible to determine whether Kynzee's race and social class informed her respect for the host culture or whether her respect for the host culture or whether her empathy and human-ness helped her develop 
an awareness of her cultural identity. From a post-modern perspective, it is possible, even likely, that both informed her socio-cultural subjective reframing (Slattery, 2013). From my observations and analysis of the data, I maintain that Kynzee's Latina identity and search for heritage expedited the process of socio-cultural transformation. Yet, I additionally posit her personal characteristics of respect, permeability, and empathy were the most powerful agents to bring about Kynzee's subjective reframing about the negative impact of United States gun culture.

\section{Participant Eight—“Tess"-Coming Home to a Place I've Never Been Before.}

Tess was a twenty-one-year-old student between her Junior and Senior year at PSU at the time of the study-abroad program. Her father was a Spanish man adopted by American parents in his teens when his parents died in a tragic accident. Tess's mom was from Ireland and came to the United States in her early twenties. Both of her parents spoke Spanish in the home and Tess was not exposed to English until the age of three. When I asked about her background during the entrance interview, Tess explained: I usually put Hispanic, and it says, "Not White...." I identify more with the Hispanic side.... I just feel like ... my family, my culture, like the way that we are, is way more Hispanic culture than it has ever been Caucasian culture in any form.... [My parents] both thought it was really important that we only spoke Spanish in the house, and they...you know, both worked for that, even though I'm only half.

However, Tess never was taught academic Spanish until college. Dr. Green explained to me that a person who speaks Spanish in the home, but has not learned academic Spanish 
is referred to as a heritage language learner. To be considered a native speaker, a person must also have schooling through high-school-level Spanish.

Tess grew up (and lived during semester breaks) in a neighborhood of her major Midwestern city colloquially and discriminatively labeled "mini-Mexico." Tess hated that nickname because her "inner-city" (Tess's words) area was home to immigrants from "all over Central and South America, not just Mexico." Consequently, Tess had a unique form of Spanish that had a Peninsular Spanish accent (from her father), infused with Latin American Spanish vocabulary (from her friends), with many English words mixed in (from her mom and school). I wrote about her Spanish in my field notes during the first week of the study-abroad program:

Tess's Spanish is very interesting. Her grammar is not very good, her vocabulary is not vast, she mixes in English words, but you can tell that she ... is a heritage speaker. She seems to understand almost everything, and she defends herself well. Her accent is part Spanish, part Latin American, and part Yanqui [North American English]. It would be fun to do a linguistic study on her. This all matches how she says they spoke Spanish only in the house as a little child, hangs out with people from all over Latin America, and her Mom is Irish. She also went to school in the United States. Therefore, her grammar and accent are perfect in English. She is very comfortable, defending [and explaining] herself in Spanish, though.

Tess was the oldest of eight siblings and her parents struggled to provide for their large family. She said she was told on many occasions by teachers and school 
administrators that they lived "below the poverty line." Despite their poverty, Tess did not feel like she "missed out" on anything in her upbringing. On the contrary, she had fond memories of the sense of community growing up in her family. In the entrance interview, Tess explained about her neighborhood:

It's a community [where] everybody looks out for everybody and ... everybody tries to help everybody out. It's not just like, “Oh, you're struggling. Well, that sucks...." We always cook food for each other, and we're always hanging out, like we make food for our neighbors, like if you have extra, you take it over to 'em.... If you have the opportunity and you see something that ... your neighbor kid would like, you get it for 'em.

Likewise, in the member-check interview, Tess said: When I'm back home [from PSU], all of my friends speak Spanish ... have huge parties with our families.... Our families get together; our families are friends.... [Although] we're all from different countries and our cultures are all a little bit different ... it's as close as it gets to having people that ... have the same culture.... We all have ... weekly barbecues with our families.... We go out together. We pretty much spend all of our time together. It's like family. Tess also loved being raised "Spanish" and remembered her father's pride in his culture and country of birth and native language. However, due to a string of difficult job losses, Tess's father joined the United States military. According to Tess, her father came back from his first tour in Iraq "whitewashed" as an "American." He never wanted to speak Spanish after being sent to Iraq. Tess's father turned to alcohol to self-medicate 
against the effects of Post Traumatic Stress Disorder. According to Tess, he went from being a lively, compassionate, and loving dad to a mean and violent person when he drank. Soon thereafter, her parents divorced, split up the large family, and Tess claims she did not know who he was after that. Until just prior to the study-abroad program, Tess and her father had not spoken to each other for over six years. Fittingly, Tess's main impetus for going to Spain was to connect to her heritage and celebrate her fond memories of who her dad was before going off to war.

Just before the study-abroad trip, Tess called her dad at her mom's request. In the member-check interview, she recounted the discussion:

The reason I had seen him and he knew I was going on this trip was because I had cancer and my mom thought it was important that he knew.... I disagreed, but I went along with it anyway.... And that's how I told him about it.... And he's asked, not me personally, but he's asked ... my little brother ... how it went, and was very excited, from what I heard, to hear [my brother] regurgitate the stories.... I wanted to be able to reconnect with him, but ... he's not the same person.... Even though he seems like he wants to reconnect, I'm not really sure if he would.... I don't know, it's a really complicated situation. Like, I ... just saw him [at my dad's adopted dad's funeral].... I spoke to [my dad at the funeral, but] that was the first time I'd really talked to him, [which] was about a week ago [five months after the study-abroad program], when my grandpa died.

To what extent do seven students experience the elements of transformative learning during a month-long study-abroad experience in Spain? To discuss culture 
shocks with Tess is impossible. Rather than cognitive dissonance, Tess indicated experiencing cognitive consonance. Spain made sense to her and the culture of her host family and friends helped her to understand her own heritage and to develop a positive identity about her upbringing. Repeatedly, even daily, I overheard her say comments like, "I love everything!" and, "I'm just so happy to be here!" Furthermore, the term culture surprise even does a disservice to Tess's experiences. Tess repeatedly described a feeling of "coming home" to Spain, even though she had never been there before. In fact, moving a hundred miles away from her big city in the Midwest to the small town where PSU was located created more cognitive dissonance for Tess than traveling thousands of miles to Spain. In the entrance interview, she described the culture shock of PSU:

[PSU] is so different from what I'm used to.... It's such a small city, and there's not much to do. And the people, the culture's really different. I don't have any friends that are Hispanic, really, in [PSU town], that like have the same kinda like way of living, like I do.... It's completely different ... like the customs [and] norms that people do.... I didn't learn until I came to college about ... what's "normal" for families, because ... my family is just...really, really different in the way that we do things compared to the way that they do things....

Moreover, Tess explained in the interview that she felt more comfortable with her host family in Levante than she did with her "American" roommate at PSU:

My host-family compares to my normal family. It feels more like being at home than being at school feels like being at home. The way that my "mom" and my host "brother" talk to each other is the same way that me and my brothers (sic) 
will talk to my mom.... The family dynamic is the exact same.... The apartment even looks similar. The food is very, very similar to what I grew up with.... Everything from the verbal communication to the physical communication, like body language, is so similar to the way I was at home.

Additionally, Tess felt like the values of the Spanish were closer to her family than "American" values were:

I feel like [Spain] has more of a sense of community that the United States usually doesn't have.... [People in the United States are] so individualistic and ... my family's not like that. And then, when I come here ... it seems so much more like being at home.... When we're walking in the street, it doesn't matter if you know somebody or not ... you say "Hi," and ... you can have an actual conversation. When we ask someone how they're doing, we're not just asking them, like, "Oh good," and then you want to leave and you get annoyed if they try to tell you how they're really doing. Like, we actually want to know.... Everybody [in Spain] is so friendly and nice, and it seems like ... it's just completely different [than the United States]. You can have full conversations with people you don't know here, and ... it's a lot more like home does. [PSU] was a real...eye-opener, culture shock ... which is weird, 'cause you wouldn't think it would be. But I had bad culture shock [at PSU]. I went home every single weekend when I first started school, 'cause ... it was so different, and people were so cliquey, and they don't ... talk to new people, and I had a really hard time making friends. [Whereas in my city and Spain, I] made friends every day. [At LU and PSU] I spend every 
single day with the same people in classes, and I don't know anything about them. And I try talking to them, and they're just like, "Oh, I have my friends," like ... "You're not part of it."

During the member-check interview, I pointed out to Tess that she often commented on how "nice" Levantinos were, while many of her classmates felt culturally and linguistically "othered" (the students commented out loud about it frequently in Tess's presence, so I was not violating any researcher/participant confidentiality). I asked Tess why she felt there was a contrast. She replied:

I don't know if it has something to do with ... [the fact that] I'm more used to culture, or maybe $[\mathrm{I}]$ was more comfortable with the language.... I was able to have more conversations with people, and there [were] no misunderstandings, where I would have thought that somebody was saying something that they weren't, or that they were judging or rude when maybe they weren't....

Everybody I met was so nice.... I would meet people on the side of the street and just have conversations with them.... I met a lot of really amazing people and I got along really well with them.... Maybe they didn't understand what people were telling them, or they just ... went into it assuming people would look down on them, and so they noticed it... [In contrast,] I got along with everybody really well. I thought people were really friendly.

Program surprise: Loosely structured. One "cultural" surprise with the LU study-abroad program was the loose structure of the program. When I asked Tess if there were any surprises or shocks she experienced during her month in Spain during the exit 
interview, she responded:

I was surprised that the trip wasn't [extremely] structured. I'm really happy it wasn't. Very pleasantly surprised, because I ... didn't want it to be like, “Here, let me hold your hand...." I wanted it to be like living here like a normal person.

She repeated liking the loose structure of the study-abroad program during the membercheck interview:

I was really happy that I got to choose ... how I got [to Spain], and getting there by myself, and having the opportunity to do it alone, 'cause if we'd done that whole part as a group, I wouldn't have had the same experience when I first got there. Because, one of my favorite things about when I first got there was not only meeting my cab drivers and having hilarious cab rides, but also on the train ride to Alicante, I met two people on the train and we talked the entire five hour train ride, and I got really close to them.

Culture surprise: Bullfight and community. Although Tess did not like the killing of the bulls during the bullfight, she further noticed the sense of community, culture, and unity through chanting at the bullfight. She maintained that it helped her “connect with being Spanish.” In the exit interview, Tess stated:

I really loved ... when we first walked up [and saw] all the people chanting. Everyone was together, and $[\mathrm{I}]$ could really feel the community and the culture, and ... I loved that, 'cause it was like, one of those times where I really, really, really felt like I was part of Spain.... That helped me ... connect with being Spanish, and being [in Spain]... It ... seemed really inhumane and...depressing, 
watching them slowly kill the animals. But at the same time ... I love how I saw ... the same ... costumes/uniforms [as I did growing up with my father from Spain]. [I could] really feel ... [I was] in Spain.... I know my dad used to do it when he was a kid.... It was like, my family.... He used to go with his family when he was younger ... and he'd tell us about it, and so it was ... really cool to actually see it myself.... I don't think I'd ever do it again, but I don't regret having the experience.

In the member-check interview, Tess reiterated that she felt connected to her heritage stemming back to ancient Rome and expressed sadness that bullfighting may not be around in another generation. She explained:

Some [PSU] people left, and ... as much as I didn't want to watch them kill the animals ... I didn't want to leave, because I was really enjoying... being there in the atmosphere.... As ... culture ... grows, and gets ... [more] globalized ... where everybody is pretty much ... [the same], Spain has had [bullfights] for hundreds of years. That hasn't changed, and they haven't changed how they do it, either.... They still wear the same costumes ... it's done the same way ... as it was when my grandparents watched it.

Tess also found her classmates' lack of historical perspective disappointing:

They were like, "Oh my god! This is horrible!" I'm like, "Now just think ... hundreds, [even] thousands of years ago ... they used to put two people in this ring, and make them fight to the death.” I was like, "That's a lot worse!” ... I was like, "So, this is horrible, yes, and I hate the [bulls'] breathing heavy and you can 
tell that it's in pain, and there's blood everywhere. But ... at least we've come farther than that.

During the bullfight, Dr. Green explained that he took students to the bullfight because "my students' children will probably never have the same opportunity." Furthermore, he added that two autonomous communities (similar to our states) in Spain have already outlawed the practice and more were legislating it at the time of the study-abroad program. Additionally, Dr. Green stated he felt that seeing the uncomfortably violent bullfights was also a way to encourage his students to reflect upon the ugly and violent aspects of culture in the United States. It appeared from Tess's data that her thoughts closely paralleled Dr. Green's reasons for encouraging the students to attend.

Cultural surprise: Festivals. One of the questions that I asked all of the participants in the exit interview was to recount their experiences and reactions to the festivals. Tess, more than any of the other participants, got excited in her body language and tone of voice as she discussed the festivals. Without even having to think about the question, Tess interrupted even before I could finish asking the question:

First of all, [the festivals are] amazing, and the United States sucks, 'cause we don't do anything like that. If you want really honest, brutal reaction ... the city I live in ... is bigger than Levante. And our festivals aren't nearly as good as that. That was awesome.... [Secondly,] I saw [families celebrating] here, and my family could be doing the exact same thing.... I mean, not that they live in Levante ... but ... having huge family reunions like that, where everyone's just eats and dances. 
TRAVEL FOR TRANSFORMATION

Transformations. During the first week of classes, the participants went on a tour of the Castillo de Santa Bárbara. Prior to the study-abroad program, Tess felt like a "part of [her] was missing" and that she participated in the study-abroad to "really connect with the culture and people" of her father's country of origin. As I observed the students, most of them appeared bored from their body language (and did not even seem to be paying attention to the guide's detailed explanations about the history of the castle). However, as I looked at Tess, I could see that she was tearing up. I approached her and asked her what she was experiencing. Tess replied:

When we walked into the castle, [the guide] was showing all of the families' names and their crests ... and [the families that] conquered the area, and built the castle, my family's name was on the wall.... And I ... started crying, 'cause ... I came here to try to connect with who ...my family ... was, especially with everything with my dad now. I really, really felt like I needed that part of who I am, and to find that. And ... to walk into ... an actual castle, and my family's crest and my name is ... written on the wall! I was like ... "This is ... where I'm supposed to be." If that wasn't a sign, I don't know what was.... It was amazing.... It was like feeling like I was ... at home.... We're descendants of that ... no matter how far down it is. But ... the fact that my family descends from some really amazing great people that have done really big things in the country...it's awesome. And it's definitely emotional.... I was very surprised. I didn't expect that." 
Tess had a similar experience when we toured the cathedral in Granada where Ferdinand and Isabella are buried. Tess again began to cry as she stated she felt that she was experiencing what formed "her country" and "her people." In the exit interview, I asked Tess if these experiences changed the way she saw her heritage. She answered that the study-abroad program "solidified [her] cultural feelings" and that she "realized way more that [Spain] is where I'm supposed to be and I belong, instead of in the [United States]." Tess further clarified this sense of belonging:

I don't know if it's really a change in the way I think, as more of ... feeling the liberty to ... be myself.... In the United States ... there's lots of things about my personality that I have to suppress... [For] example, ... I'm really touchy. [In] my family ... we like human contact. Not in ... a weird way. Just ... we like to touch people on the arms, give people hugs [and] kisses.... And, when you go touching white people in the [United States], they freak out.

Furthermore, in the member-check interview, Tess reflected on this opportunity to experience her heritage and connect with a part of who she was:

It wasn't different, it was just more me.... I was definitely more enjoying life, and especially after going through such a dark time before I went, coming back I was very happy and very ... me.... I felt like I was finally able to really see where I come from. There [were] a million things where ... our teacher was telling us about things that were "Spanish-isms" that were just kinda like weird, quirky things about the Spanish ... that I realized $I$ do.... There [was] definitely a lot of personal growth, and ... connecting with heritage and family.... I realized a lot 
about myself, and being able to be myself the whole time.... I felt like I was becoming more and more ... comfortable [with] myself the whole time I was there.

After having time to reflect on the way the study-abroad program impacted her life outside of school, Tess further explained that the study-abroad program was a way for her to heal from the physical and emotional battle with cancer that she overcame just six weeks before the LU program. She emotionally explained in the member-check interview:

[Living in Spain] definitely ... impacted my life.... I'm ... a lot more grateful for everything that I have.... I really couldn't afford [the trip] and I made myself able to afford it.... I worked so hard to get there, and the whole reason ... I wanted to go so bad [was] when I had cancer, to help me get through it, [I] was thinking about going to Spain.... I just was like, "When this is over, I'm going to Spain, and I'm doing that for myself, and I'm getting away for a month, and I'm going to ... connect with myself, and heal from the emotional toll that cancer has on you." Because, when I was [in the United States], I was still working when I had cancer, and I still had classes, which was the worst idea I've ever had.... I never really got a chance to emotionally heal from it because ... I had ... cervical cancer, [which is] such a ... personal kind of cancer.... It ... like breast cancer, testicular cancer, prostate cancer, hits you in a different kind of way. It's not just ... battling for your life, but it's like losing a part of who every human being is.... Like your sexuality, in a way, you kinda lose that, in having such a [personal] kind of 
cancer, [and] it ... dehumanizes [you].... That trip was for me to really be able to connect with myself and emotionally heal from everything that I did with cancer. It was evident that, for Tess, this trip was much more than a chance to rest and heal from cancer, however. This trip was an opportunity to find her roots. I maintain Tess developed a sense of identity by spending a month in Spain that will continue to impact her life long after the study-abroad program. She explained in the member-check interview the connection she felt to the people of Levante:

Every single day, I miss the people. I miss the way of life. I miss walking in the streets, not just for walking's sake, but the fact that you can just walk through the streets and there's always people out walking, and the culture, and just listening, and smelling smells, and ... just being there.... It's probably the [small] things I miss the most. It's not any of the huge trips, 'cause I loved all of those, but it is more the every single day, just getting the opportunity to walk down the street and just see all the other people ... living their lives, and ... seeing the art that was everywhere, and just the culture, the people, the way the people were ... is definitely ... what I miss the most. And I miss that on a very regular basis.... I miss being able to speak Spanish everyday. I miss the food, and the music.... Everything about it... I miss the architecture... I love being myself. I love how touch-y everybody is.... And being able to approach random people on ... the subway? You can't do that here. \{laughs\} You can't walk up to random people and be like, "Oh hey." Public transportation! I miss that a lot, too. 
More than any other participant, Tess felt a sense of radical cultural inclusion or a sense of "coming home," even though she had not ever been to Spain before. She repeatedly discussed how Spain reminded her of her dad before he went into the United States military. Her cultural inclusion, combined with the cultural "otherness" she felt in the United States will be discussed in the section on cultural and linguistic "otherness" below.

Other: Reflection through dialogue with researcher. As I was about to hang up the phone from the member-check interview, Tess took a moment to thank me for giving her the opportunity to reflect upon what the trip meant to her. She informed me that having "a listening ear" helped her process the thoughts and emotions she experienced throughout the study-abroad program. This was a revelation to me as the researcher because I always tried to be sensitive to the participants' time and was grateful for their openness and transparency throughout the process. Tess said:

Actually, I wanted to thank you, because ... you keep saying, "Thank you for your time," but honestly, being able to talk to somebody who's all-ears and wants to listen was really, really helpful, I think to, not only me, but probably all of us, to be able to talk about our feelings and how we felt about [the study-abroad program].... That's definitely something I appreciated, even now, being able to reflect back on it and think of everything that happened, and, when we were there, being able to put it all on paper, and talk about how I was feeling.... Nobody really related, and I definitely feel like you related very well to a lot of the stuff I 
was going though ... as well as just having somebody who wanted to listen, was very nice.

I thanked Tess for the kind words and told her, "I wish it was completely altruistic, but I'm also getting a degree out of this." To which she responded:

It was a mutual benefit. It wasn't just like a one-way thing. It wasn't just like you taking my time. I wanted to make sure you knew that it was ... really helpful to probably all of us to talk to you.

Tess's comments led me to question one of Mezirow's (1996, 1997, 1998, 2000, 2012) primary assumptions about cognitive dissonance and its role in bringing about critical reflection. Mezirow maintains that cognitive dissonance (being confronted with an idea or experience that contradicts a prior epistemological assumption) or a disorienting dilemma (a situation wherein intense emotions are brought about through inner conflict as a result of a major life transition) is necessary to bring about critical reflection. However, Tess did not state any intense inner conflicts or major life transitions during her time in the study-abroad program. Rather, Tess was extremely comfortable in her settings and felt like she was "home" for the first time in her adult life. However, Tisdell (2012) states that transformative learning often happens years after the transformative event.

My grapple analyzing Tess's data is whether the intense life transitions of cancer, estrangement from her dad, and then a metaphorical reconnecting to her dad's childhood were what would be considered a "disorienting dilemma" under Mezirow's (2000) theory. From the data collected from the study-abroad program, it is impossible to 
decipher which of the events (if not a combination of all of them) led to Tess's transformational learning as she came to a sense of cultural identity. Moreover, K. Taylor and Elias (2012) caution that although intense, disorienting dilemmas are not always negative. However, it appears from the data collected for this study that in respect to cognitive dissonance and disorienting dilemmas, Tess experienced these before, not during, the study-abroad program. In contrast, during the month in Spain, Tess experienced what I earlier labeled cognitive consonance, rather than dissonance. A suggestion for future study would be to investigate the role cognitive consonance plays in critical reflection, which according to Mezirow $(2000 ; 2012)$ and others (Brookfield, 2002; Ettling, 2006) is a precursor to transformative learning.

Prior research regarding travel for socio-cultural transformation may offer some insights into Tess's experiences in Spain and critical reflection, however. Tess's experience with critical reflection appeared to have been brought about by the travel experience itself, rather than through a disorienting dilemma. By stepping away from her socio-cultural context at PSU and home, Tess was able to have space to critical reflect upon her life (Coghlan \& Gooch, 2011). While at PSU, Tess took over 20 credits a semester and worked 60-80 hours a week. It is possible that increased time to reflect upon her life may have given Tess time to critically reflect upon and understand her place in society and what she desired moving forward. Furthermore, Pritchard, Morgan, and Ateljevic (2011) state that travel and tourism offer time for reflection of one's values and one's place in the world that formal classroom studies do not. I posit that Tess may have never had space to reflect upon her cognitive dissonance in the United States without the 
time and space given in the study-abroad program. Additionally, prior research indicates that opportunities for reflection and contemplation during (Morgan, 2010), and after (Ross, 2010) study abroad have been shown to aid a traveler to reflect upon and extract meaning from travel. These findings align with Tess's statement that being able to reflect on her experiences during the study-abroad program and several months after may have augmented the opportunities for critical reflection.

\section{What role did encounters with cultural and linguistic "otherness" play in the} transformative learning (or lack thereof) of each participant? Among the studyabroad participants, Tess's sense of inclusion and "otherness" had an inverse relationship — she felt "at home" and included in Spain, but experienced culture shock and "otherness" within dominant United States culture. Furthermore, Tess experienced “otherness" from the PSU study-abroad participants during the study-abroad program.

Linguistic and cultural "otherness" in the United States? Tess felt like she did not "fit in" in the United States. She had light skin, yet was a heritage Spanish speaker. She spent much of her time with other young adults from Central and South America, yet they made fun of her for her light skin tone. Tess did not see herself as "White," but rather as "Hispanic." However, she understood that her light skin tone allowed her to be socially constructed in society as White. In contrast, White students at PSU labeled her "a dirty Mexican," which Tess described in the exit interview as:

completely wrong on every level, but ... just because ...they ... don't understand ... the differences between being Mexican and anything other than Mexican.... I'm so different than they are.... I've never been able to quite mesh into that ... 
perfect little ... thing that they have. I don't understand it. I never will understand it. I've always stood out because of it, and it's really hard to ... live [at PSU] ... and always feel like I'm ... going to be an outsider.

Following a conversation of her social construction of Whiteness, due to her light skin, but her cultural identity as Spanish, I wrote the following about Tess in my field notes:

Tess talked about the racism she gets from both Latinas/os because she looks White and from White individuals because she speaks Spanish. She doesn't really feel "home" with either group, but [in Spain, she is with] "her people." She has seen tons of people like her here [who have light skin, but speak Spanish as their native language]. She could pass as "White" easily in the United States, due to the way she is racially constructed by others. However, she identifies much more with people of color from her neighborhood [in which she grew up] and her Spanish-speaking heritage and family culture.

Common to many people of color who can pass as White in society, but who culturally and socially identify as a person of color (Harris, 1993), Tess internalized her oppression and choose silence over disrupting or reflecting upon it, because it was too emotionally exhausting to challenge oppression from all of her peer groups. In the member-check interview, she explained:

There [were] a lot of things ... where I ... internalize it when I'm here in the [United States], because it's stuff that people don't really understand. And, when I was [in Spain], I felt like ... parts of $m e$ that are really, really, really important, 
strong characteristics of who I am as a person ... I was able to feed those other parts of ... my self.

Furthermore, I observed (although she never explicitly stated it) that Tess felt that confronting the racism, classism, and linguicism that she faced in the United States would simply lead to further marginalization. In fact, I observed a situation in which increased marginalization occurred from the participants of the study after Tess tried to challenge an incorrect narrative about her United States neighborhood (discussed in the following section). Moreover, from analyzing Tess's data it appeared that the capacity to reflect upon her marginalization opened Tess up to acknowledge the intersecting racism, linguicism, and classism she dealt with regularly in the United States. Furthermore, Tess described this opportunity to "be who she really was" during her month and a half in Spain a "healing process."

Perceived "otherness" from PSU participants, not from Levantinos. Within the first couple of days of the study-abroad program, I was surprised how much socioeconomic data came from the students as they described their hometowns. Especially for the students who were from the wealthy suburb of the major Midwestern city, they discussed their location in direct contrast to the city and often used Tess and "Little Mexico" as the binary polar opposite to the wealth of their suburbs. Hearing words like "ethnic," "inner-city," "urban," "poor," and "minority" were common ways they used to contrast their upper-middle class, White suburbs from that "other" place where Tess grew up. I was caught off guard by the degree of classism and racism coming from the participants, especially before I felt that I had fully (or even partially) gained their trust. I 
wrote in my field notes:

It is interesting how much socio-economic data comes out of them telling me where they are from. I found out [from the other participants] that Tess is from what sounds like "inner-city" or "urban" [Midwestern city] while the rest [of the participants] are from country towns and suburbs.

As a researcher, I remember reflecting on how much their comments reflected the CRT critique of Whiteness (along with middle-class values) being normalized within United States culture (Ladson-Billings, 2009; Leonardo, 2009; Tatum, 2009). I noted that Kristene (a pre-service teacher), Becky (a pre-service teacher), and Jennifer (a political science major) used more "polite" language to describe the differences between the "inner-city" and the suburbs. Rather than saying words like "ethnic," they would use terms like "diverse" or "multicultural." However, the contrast of socio-political binaries of Black/White (from Ladson-Billing's [2009] CRT definition), safe/unsafe, and innercity/suburban permeated the explanations of even the "polite" students' hometowns (reinforcing Delgado's [2009a] position that even liberal narratives support White supremacy).

One example from the bus ride home from Granada (one week into the studyabroad program) illustrates the concept of social privilege - in contrast to Tess's lack of social capital — as a predominant theme among the students. I overheard a conversation among Kristene, Amy (Kylie's friend who was not studied), Kylie, Becky, and Anne about the different neighborhoods of the major Midwestern city near which they all lived. The participants talked about the "good" and "bad" areas of the city (and its 
accompanying suburbs). However, I wrote that Anne's speech was especially full of discriminatory, deficit viewpoints using the following phrases: "drug users," "murderers," "no one should ever go to that part of town," "get raped/assaulted," and, "unemployed." All of the participants used words like "unsafe." At the time, I could not tell whether Tess was thinking about something else or listening uncomfortably. From her body language, it looked like the latter. Tess eventually interjected with, "You don't know what it is really like." However, I silently questioned if one of the areas full of "bad" and "unsafe" human beings was Tess's neighborhood. I could not tell whether she was joining in as a way to protect herself from further marginalization from the group or as a defense of her neighborhood.

Because I did not want to negatively impact Tess's relationship with the other participants, I waited to discuss this incident with her until the member-check interview. I asked Tess if she also overheard that conversation. She replied:

Yeah, I actually remember it very well. I also remember when we were in $[\mathrm{el}$ Castillo de Santa Bárbara], one of the first days, getting in an almost-argument with somebody, because of ... similar [narratives].... I was definitely ignoring it so I didn't get irritated."

Tess also indicated that she was attempting to ignore the fellow participants because she was avoiding a physical or verbal altercation, which she felt was inevitable if she challenged their "othered" narratives about her community. Then, Tess continued with the Castillo story that troubled her even more: 
When we were at el Castillo, [Amy] was making some remark about how ... she was from [Tess's Midwestern city], but she isn't.... She's from [a wealthy suburb], and it's a very rich area. And, she said something ... dismissive, and then was like talking down on the "inner-city," and "people like that," and it really bothered me. This was when I got very irritated, 'cause I was like, "You're not from [Midwestern city], like don't say you're from [there,] 'cause you're not [Tess's voice sounded angry and tense recounting this part of the story].... I got very irritated at the way she was talking about people from the "inner-city," but still trying to ... trying to classify herself as from [Midwestern city], because it's completely a different atmosphere, it's a completely different area. But [I] was just like, "No, you're not.” And ... I definitely had certain [times] where I just felt like people ... looked down on me "cause I was from a different situation. And, personality-wise, you can very much tell, [I'm] from a different socio-economic ... status than everyone else that was on the trip.

As I reviewed Tess's interviews and other data, I realized that many of the PSU students interacted with Tess in much the same way that they interacted with the host culture - they exploited her and appropriated her culture when it worked to their benefit, but had little observed or stated desire to give back to Tess, the host community, or to "other" communities in the United States upon return. For example, they often "included" Tess when she had access to activities, such as parties, where Levantino college students (rather than tourists) would be present. In contrast, during the cultural excursions, where they did not need Tess's access to the host community, I often observed Tess standing 
alone (the exceptions to this pattern were Becky and Kynzee, yet they seemed equally willing and capable to interact with the dominant group or Tess). From Tess's viewpoint, this "othering" from the study-abroad participants was a continuation of the daily racism, linguicism, and classicism she experienced at PSU. Tess's "othering" and appropriation from several of the PSU participants aligns with the CRT concept of interest convergence (Delgado, 2009b; Gillborn, 2009; Ladson-Billings, 2009). This topic will be analyzed in greater detail in the section on interest convergence below.

Catalyst of radical inclusion: "Paco." From the beginning, Tess commented on how her host family culturally reminded her of her family growing up before her dad entered the United States military. In the entrance interview, Tess described her host "mom:"

My "mom" is like a lot younger [than the other participants' "moms."] She's so much like my mom. And ... the family dynamic's like mine, and I was surprised by that. I'm really happy about it, 'cause it's like, I fit in so well, and [we] can just sit down, and ... there's no ... stress.

Tess also described the excitement to have a host "brother" who went to LU and was eager to include her in his daily activities and introduce her to his friends:

I've got a host "brother" who's two years older than I am, and he goes to the same university.... He has been driving me to school.... He specifically sets his schedule so that he can drive with me to school.... I've gotten to meet some of his friends ... which was awesome. That's what I want. I'd rather hang out with people from Spain. 
About ten days into the host program, I ran into Tess and her host "brother" walking to class. I wrote about the encounter in my field notes: I saw Tess walking with her host "brother" to class. She introduced him to me (Paco?). I asked if he was her host "brother." She said that that was a "really weird" and "really icky" way to think about it. This [encounter] makes me wonder if there is a little more to this relationship than hospitality. She has mentioned going out to bars with him, him driving her to school every day, and him "accidentally" seeing her in her bra and underwear. That was all in the first week [before the entrance interview]. I wonder of the relationship has progressed, but also want to be careful in how I approach it.

On the third Monday of classes, I held an "official focus-group interview" (following Dr. Green's weekly check-ins). I asked the LU professors if I could write the questions on the board. One of the questions was to ask how relationships with their host families were going about halfway through the program. I wrote the following in my field notes: Tess turned red and said the one about host family relationships might have to be in a personal interview (I am guessing that the thing with the host brother might have turned into something).

In the exit interview, Tess and I discussed her sense of cognitive consonance, rather than dissonance, and her feelings of radical inclusion. She indicated that her relationship with Paco had a positive impact on her sense of inclusion:

"I think I experienced [Spain] a little bit different than everybody else, because of Paco...." Me and Paco (sic) have got[ten] ... really, really close. Me and him 
(sic) hang out all the time.... I've gotten to see how a Levantino ... twentysomething, university-aged kid would live. Because ... we go out with his friends all the time.... We go to the restaurants that he would go to, go do the things that he would do... [I saw how a] student who lived here would actually [live.]

In addition, Tess described the pair going to family and friend parties, restaurants, "dive bars," soccer games, night clubs, block parties, and other "local college student" activities. In the member-check interview I asked Tess if she felt her relationship with Paco was a benefit or a limitation to her stated goal of cultural immersion and discovery. She answered:

I think it was extremely beneficial.... I think it definitely helped, because a lot of other people in our group ... complained all the time about not being able to really connect with anybody [their age] while they were [in Spain], and they had trouble meeting people.... And I was extremely lucky in the fact that I had [Paco] to show me around and show me what it's like to actually live there and not just go to the touristy things. 'Cause the people in our group, whenever they'd invite me out, a lot of the times they were going to ... [a British department store] or ... a really touristy bar, or something like that.... They didn't really go to any of the... actual Spanish places where Spaniards would really go.... And [Paco] took me to some really amazing places that I know a lot of other people didn't get a chance to see 'cause it's not one of the touristy-type places.... For me, ... I didn't want to go see the touristy shops and buy little knick-knacky things, I didn't want to go to the super-touristy beach and just hang out on the beach all day, or things 
like that. .... I really wanted to get the experience of what it would be like to actually live there, and I think that $[\mathrm{Paco}]$... fully helped ... complete that whole image of what it is like to live there.

I followed up asking Tess to imagine that she never met Paco. I asked her how her studyabroad program and cultural immersion goals might have been different. She replied: I would've tried to meet people as much as possible ... and I definitely felt very comfortable going to [authentic] places ... by myself.... But it was easier to know where to go.... I would still try to go to those places, but it's just harder to know where they are.... And it would have been harder to make those friendships, because when you just walk down the street and just run into some random person, it's harder to become close the way that you do when you're actually living with somebody. So, I feel like having him there, and the fact that we were actually ... living in the same place, made it easier for us to get really close.... I still would have tried.... I ... definitely would've still done the same stuff, but ... I don't think it would've been as successful.

Like Tess, I feel that Paco was a catalyst that sped up Tess's cultural immersion and inclusion. Yet, from her comments and her stated desires to experience Spanish life, I believe Tess would have taken advantage of every opportunity to experience the "real" Spain (at least real for her), whether she met Paco or not. For example, Tess went to Barcelona for a week before and a week after the LU study-abroad program. In Tess's data are dozens of stories of her meeting and having long conversations with people in trains, taxi drivers, individuals she met on the street, and others in bars and discotheques. 
She continued to meet and become acquainted with people during her whole trip, not solely during the time she spent with Paco. Therefore, I agree with her observation that she would have sought out, maybe not as efficiently, authentic opportunities to discover what "real college students live like in Spain."

Radical inclusion and relocation? Tess's sense of radical immersion reaffirmed her desire to return to Spain to teach English after finishing college. In the membercheck interview, I reminded her that she indicated in the entrance interview that she wanted to return to Spain. I asked her if she was still planning on returning. She replied, “Oh yes. Very much so. That's my plan.” I followed with, "Do you feel that your experience in Spain either solidified that [desire]?" She answered:

Yes, of course.... Being there for a month and realizing how much that is who I am, how much I fit in, how much happier I was ... I was probably the happiest I've ever been in my life when [I was] there.... I wasn't even doing anything special: ... just everyday things, like going to class ... eating with a family ... walking around the city ... going to the grocery store, [but] I was just so happy.... It definitely solidified that fact that I know that I'm supposed to be there. Reverse culture shock. Stemming from her sense of radical inclusion while in Spain, Tess experienced reverse culture shock upon returning to her more-than-full-time workload and academic credit load. She exclaimed, referring to this shock, "America sucks!” She also described her intense workload in the member-check interview: I have three jobs. I work 80 hours a week in the [United States] and I never have time to do anything.... I don't get any support from my parents.... I have an 
apartment in [Midwestern city], I have a house in [PSU town], and then I pay for ... all my school stuff. And so I have, like, no money whatsoever. And, I always feel like I'm drowning.

Although Tess had kept up this lifestyle before the study-abroad program (while battling cervical cancer and taking twenty-two credit hours), it seemed like too much to bear after finding her identity and enjoying the lifestyle of Spain:

It was so hard to re-adjust into it and cope with [returning to my busy schedule], even though it was something I'd done my whole life ... since I've been old enough to work.... Just because...I want to be able to live that life where ... you can do...eight hours of sleep, eight hours of work or school or whatever, and then eight hours of spending time with your friends and family and doing things that you want to do.... [That] is what a schedule should look like for five days, and then [have] a couple days off. That completely to me is how you should live your life instead of working so much. And, so, going back into having absolutely no personal time, or time to do things that I wanted, ... [or to] explore more ... personal growth [from Spain], and instead I went straight to work every single day.

Several of the participants (Jennifer, Becky, and Kynzee) also identified and noted this difference between their perceptions of Spanish and American lifestyles. They learned (and agreed with) the common Spanish phrase that "Americans live to work, while the Spanish work to live." However, for Tess, a return to a lifestyle of extreme work was untenable after enjoying a summer of relatively fewer professional and 
academic responsibilities. She saw no way out of this intense work schedule in the United States and excitedly anticipated a return to the country where she found her cultural identity.

What is each student's racial, economic, gender, and cultural identity? How does each student's identity impact her worldview and their transformations (or lack thereof) during the study-abroad program? A major component of critical social TLT is to recognize and attend to historically under-represented voices through research. Johnson-Bailey and Alfred (2006) argue that Mezirow's (2000, 2012) TLT model largely ignores culturally-bound or silenced students (see also Cranton \& Taylor, 2012; Tisdell, 2012). Furthermore, they maintain that experience in a socially marginalized group may be a more powerful transformer than any other component of the transformative learning process because oppression requires a person to confront a lack of social, economic, or political capital (see also Ross, 2010). Therefore, like Johnson-Bailey and Alfred (2006), it is imperative that I recognize, highlight, and honor the wealth of experience and knowledge that students who are marginalized, like Tess, brought to the study-abroad program.

In order to promote the narratives of students like Tess within TLT research, E. W. Taylor (2007) calls for empirical methods that analyze sociocultural variables (race, class, gender, and culture) within TLT research. Additionally, many TLT researchers assert that a race-centric perspective can be effectively studied in TLT research (Cranton \& Taylor, 2012; Taylor \& Snyder, 2012; Tisdell, 2012). Consequently, I have utilized CRT as a paradigmatic underpinning to this research. I stated in the literature review, 
"Critical race theory is a form of oppositional scholarship that exposes racism as normal; uncovers interest convergence; deconstructs the myths of colorblindness within educational institutions; promotes the voices and narratives of people of color; and exposes White, middle-class values as normative (Delgado, 2009a; Ladson-Billings, 1998; Litowitz, 2009; Smith \& Stovall, 2008; Taylor, 2006).” CRT is an especially important framework to highlight Tess's narrative because she identifies as Latina. Therefore, in the following section, I will analyze Tess's data through a CRT lens and will address each of the concepts in the quote above to answer what impact Tess's racial, economic, linguistic, and cultural identity had on her study-abroad experience in Spain.

Racism as normal. Leonardo (2009) maintains that racism is so deeply embedded within the fabric of American society that even people of color add to or participate in their own oppression through reinforcing hegemony or "inter-minoritarian politics" (p. 268). Tess described experiencing "othering" from both the Latinas/os with whom she had grown up (due to her light skin tone) and the White students at both at PSU and in the study-abroad program (due to her heritage language, Spanish culture, and poverty). Consequently, Tess internalized—rather than reflected upon, acknowledged, or discussed — the racism and classism she felt she experienced throughout her life. Through critical reflection and a safe space to discuss these topics (our interviews), Tess came to identify power structures that led to oppression for her, her family, and "kids where [she] grew up." Through these interviews and the intense cultural identity she developed during the study-abroad program, Tess cultivated an awareness of, and a desire to unravel, hegemonic forces in her life. Tess's personal awareness was a valuable first 
step toward dismantling the racism, classism, and linguicism she experienced daily in the United States. Nevertheless, I observed the longer I encouraged her to talk and discuss her experiences in Spain, the more Tess spread the reach of her narrative from personal grapples to oppressive institutions within society and how they impacted "kids like [her]." Consequently, I am confident that Tess's awareness of hegemony will not only influence her own life, but the lives of other "kids like [her]" as she becomes more aware and more confident speaking against oppressive power structures.

Interest convergence. As discussed in the section on cultural "otherness," Tess experienced interest convergence from fellow study-abroad participants as they appropriated her cultural capital when it worked to their benefit, but had little observed or stated desire to give back to Tess or the host community (with whom Tess passionately identified). Tess's "othering" and exploitation from several of the PSU participants aligns with the CRT concept of interest convergence, wherein any perceived benefit to marginalized individuals benefits the dominant group more (Delgado, 2009a; Gillborn, 2009; Ladson-Billings, 2009). Like the upper-middle and middle-class communities they represented, I observed that the PSU students tended to include and value Tess's cultural knowledge only when it served their personal interests (Taylor, 2009).

Whiteness as normal. Rather than an attack on the characters of the other participants in the study, however, Tess's experiences with racial, linguistic, and class "othering" by some of the participants must be understood within the construct of racial innocence reinforced by normalization of Whiteness within the United States culture. Stated differently, The PSU students' frames of reference were formed in a society where 
governmental, institutional, media, and corporate systems uphold White supremacy and obfuscate White privilege, making Tess's oppression appear normal and invisible to the other participants (Taylor, 2009). Moreover, the participants' derogatory narratives about Tess's home culture demonstrate how deeply racism, linguicism, and classism are embedded within the framework of American society (Ladson-Billings, 1998; Parker \& Lynn, 2009; Taylor, 2009). Furthermore, racism in the United States (and for the purposes of this study, when Americans are transplanted elsewhere) appears so ordinary (Delgado \& Stefancic, 2001; Solórzano \& Yosso, 2009) that the students were incapable of breaking free of their privileged worldviews to understand how their words and actions were perceived as hurtful to Tess (Brookfield, 2012). Nevertheless, any time that Tess attempted to address racism or classism (as in the story from the bus ride home from Granada), it was Tess, not the upper-middle-class students, who appeared aggressive, which further reified her marginalization. This observation parallels Tatum's (2009) position that those who confront racism or classism are often marginalized.

Middle-class values as normal. Tess manifested pride in her identity as a hard worker, even before the study-abroad program. Tess saw poverty — which resulted in her need to pay for the study-abroad program without parental or institutional support—as a benefit, rather than deficit, to her experiences in Spain. In the member-check interview, Tess explained:

I knew the second I heard about this study abroad that I wanted to do it.... Last summer I worked 80 hours a week every week in the summer, and then through the school year I worked about $50 \ldots$. I saved up as much ... as I could ... [to] 
pay for this trip. I worked really hard to get here. I spent a lot of time working and ... thinking about it... [Additionally,] I tried to spend as much as time with my family and friends that speak Spanish as possible... [because] all my friends ... and ... family speak Spanish.

I then asked Tess if she felt her hard work to pay for the trip gave her a different experience than other participants. She responded:

I think it did.... Because a lot of the kids' ... parents paid for the trip, and they didn't work at all to go. And, they've had more opportunities to go on nice, fancy, expensive trips like that.... I can't speak for them, ‘cause I don't know, but sometimes it seemed like they didn't appreciate it as much ... because it was just ... handed to them.... I definitely ... think it's different, because ... a kid like me, growing up the way I did, where I did ... [long pause] I never would have thought growing up that I would have ... an opportunity to go on such an extravagant ... trip like this. To me, staying in the hotel we stayed in in Granada was just amazing. I've never stayed in a hotel that was that nice before.... Actually getting to go because I wanted to on a trip like that ... was completely amazing to me, and unbelievable.... It's not something that ... kids that come from where I'm from usually get the opportunity to do. A lot of those kids never get an opportunity to leave the United States, [or] even the area that they grew up in. And so, I definitely appreciated it so much more. I worked my ass off to pay for that trip.... I picked up extra shifts when I was already working an ungodly amount just to pay bills.... I was so determined to go on that trip and make it 
happen, and so I felt like there [were] definitely times that people didn't really appreciate it as much.

It must also be remembered that Tess worked this hard while battling ovarian cancer and taking twenty-two credit hours at school.

Like Tess, I noted in my field notes a pattern that the more the students paid for their own trip, the less likely they were to complain about money (or, in general). Tess's statement in the member-check interview reaffirms that pattern:

I saw a very big attitude difference in certain people ... [in] how much they would complain, and they were upset, and talking about how the trip was such a letdown, and they were letting small tiny little things ruin their entire trip. And then I see them on Facebook now [5 months later] and they're like, "Oh my God, I want to go back so bad!" But the whole time we were there they were complaining and saying, "Oh my God, like, it’s so horrible 'cause Dr. Green did this, he did that, he's just ruining this whole trip." And I'm like, "If this trip meant that much to you, you wouldn't let something small like that ruin it.... You would just be embracing everything about it and not letting little things get to you."

\section{Stepping away from socio-cultural normativity as a secular pilgrimage. Tess's}

focus and hard work to connect with her identity paralleled Morgan's (2010) thesis that willingness to be transformed by a travel experience increases when there is a deep sense of purpose for personal development (Morgan, 2010). He labeled this intense desire to experience a place other than the participant's own culture a secular pilgrimage. 
According to Morgan, this feeling of being an "outsider" or "othered" that an individual might feel in a new environment increases the likeliness that a disorienting dilemma will occur (Mezirow, 2012; Morgan, 2010). Fascinatingly, Tess found her culture by studying in Spain. The "otherness" she experienced was from other participants, not the host culture. However, Morgan asserts that transformation is most likely to occur if the traveler has the opportunity to reflect upon the experience, which did impact Tess's transformation toward discovering her cultural identity.

Cultural normativity and Tess's defense of Spain. I observed because Tess felt a strong cultural connection to Spain and its people, she often fell into an "othered" position of countering the negativity and complaints she perceived were coming from her fellow PSU students. For example, many of the students complained during the membercheck interview that Dr. Green did not arrange transportation for the students to travel to Levante. Tess interrupted:

I actually ended up on the same flight as Dr. Green, and, he waved at me on the plane, and then we got off the plane, and [I] never once ... saw him [again].... [However,] I really love the fact that I did it on my own [without Dr. Green's assistance]. [So], I want to throw out ... all of the "Dr. Green's not helpful" bashing.

Furthermore, during one of the weekly focus group meetings where Dr. Green checked in with the students, one of the students discussed being upset at not having more cultural excursions. Tess countered, "You can only do so many touristy things.... We didn’t come here just to go sit on the beach." Likewise, anytime the participants expressed 
negative experiences or impressions of Spaniards, Tess jumped in with a counternarrative and a possible reason for why a Levantino might have acted the way they did in the story.

I observed that Tess's constant defense of "[her] people" led to a pattern of romanticizing the study-abroad program and Spain, however. Morgan (2010) maintains that essentialization (even through romanticizing) harms a participant's opportunity for critical reflection. Consequently, Tess's defensive position (combined with the authentically deep personal connections she was experiencing in Spain) may have limited her opportunities to critically reflect about anything negative in Spanish culture. A romanticized frame of reference (or any other essentialist stance) makes it difficult, if not impossible, to transfer personal subjective reframing toward reframing society.

However, although Tess did not have a stated goal to make the world better for others in similar situations, acknowledging disparities is a first step toward overcoming essentialism (Tatum, 2009). Furthermore, Tess did experience subjective reframing of her marginalized position within United States society and her repeated references to "people like me" makes me hopeful that this trip was a first step in having her personal transformation toward a positive sense of identity that might impact the lives of others either in Spanish or United States culture (or both).

Myth of colorblindness and micro-aggressions. Tess's experiences serve as a contrast to Tim's. Tim did not state and I did not observe any sense of "othering." However, I likened Tim's "people are people" narrative to the CRT myth of colorblindness because I argued that Tim's rejection of cultural difference may have been 
a factor that impeded him from experiencing subjective reframing about United States power structures. Furthermore, Tim's colorblind worldview appeared to limit his capacity to understand or subjectively reframe his position in society.

In contrast, Tess celebrated and honored the host culture, which is an essential component for socio-cultural reframing in travel for transformation (Morgan, 2010; Ross, 2010).. Because Tess profoundly identified with Spanish culture, it did not matter to her whether the PSU students were denigrating her home neighborhood or the host culture. Consequently, Tess received the students' ethnocentric assumptions about Spanish culture (or her background) as micro-aggressions (Sue et al., 2007). A flaw of colorblind epistemologies was as the students talked among themselves, it was evident that they all believed they similar socio-cultural frames of reference (based in the normalization of White, middle-class values). This made it impossible for them to see how denigrating Spanish society was hurtful to Tess (Brookfield, 2012; Mexirow, 2012). Rather than valuing and celebrating her cultural knowledgeable, Tess felt some of the participants "othered" her. Ironically, the culture the PSU students sought to discover through the study-abroad program existed twenty minutes away from their suburbs and sat a few feet away from them in their PSU Spanish classes long before the study-abroad program ever started. This observation led me to question if students needed to travel across the world, if socio-cultural transformation is the desired outcome of study-abroad. Rather, it seems logical that a visit to parts of town that students feel are "other" may have an equal—or more powerful—outcome in understanding and acting to disrupt social disparities (Slattery, 2013). 
Promote the voices of people of color. E. W. Taylor and Snyder (2012) posit that a deliberate and conscious strategy to employ a political framework like activism or consciousness raising must exist within TLT. Critical and emancipatory research shows a critical stance that seeks to not only understand a person, but also analyzes the power dynamics of a situation and critiques the status quo, and seeks to empower participants to transform (Merriam \& Kim, 2012). Likewise, Mezirow argues, "it is essential to seek out and encourage viewpoints that challenge prevailing norms of the dominant culture in matters of class, race, gender, technology, and environmental protection" (p. 79).

Tess pushed my boundaries of understanding the societal borderlands of individuals who lie at intersections of marginalized groups (Crenshaw, 2009). Many individuals daily navigate among seemingly contradicting aspects of self, creating a sense of both inclusion and "othering" simultaneously (Johnson-Bailey, 2012). Likewise, Tess was an insider and an outsider simultaneously in whatever community she positioned herself in within the United States. Latinas/os saw her as not fully Latina/o, while Whites marginalized her for her linguistic, cultural, and class identity. Especially within the Latina/o community, skin color does not necessarily represent cultural identity (Anzaldúa, 1990). Furthermore, it is important to acknowledge that race is a social, not biological, construct that changes with the needs of the dominant culture (Delgado \& Stefancic, 2001). Consequently, even though Tess looked White (and most likely receives unearned societal privileges based on that social construction), she has lived a life in a borderland of intersecting race, linguistic, and class marginalizations. 
Although many prefer to categorize identities into single social components, intersections of marginalizations (or intersections between privilege and marginalization) provide powerful rich new categories that need to be studied and understood (Crenshaw, 2009). Indeed, Anzaldúa maintained, "Necesitamos teorías ...that cross borders, that blur boundaries.... We are articulating new positions in the "in-between," (Anzaldúa, 1990, pp. xxv-xxvi). However, few research paradigms (especially within TLT literature) discuss, celebrate, or reveal the borderlands in participants' lives (Taylor \& Snyder, 2012). Moreover, because dominant Euro-American culture is extremely competitive, rather than cooperative, few teachers, students, and researchers feel comfortable investigating narratives outside of "us vs. them" binaries (Brookfield, 2012; O'Sullivan, 2012). This silence reifies dominant social meta-narratives and limits our capacity to understand how humans construct knowledge from their daily lived experiences (hooks, 1994; Johnson-Bailey, 2012).

By promoting Tess's narratives of her struggles inhabiting borderlands, I hope to push the academic narrative within TLT away from dualistic thinking to show the harmful effects of essentializing and stereotyping on those who experience multiple socially constructed realities (Steele, 2009, Tatum, 2009). Furthermore, I wish to highlight the exciting richness that will come as scholars embrace, study, and highlight intersectionality - rejecting pre-existing political, racial, sexual, and gender binaries to create whole new categories of understanding how humans construct their frames of reference and what motivates us to move toward critical-social transformation. 


\section{Chapter Five: Discussion and Conclusions}

Through this study I attempted to fill a gap in existing knowledge, extend past research, and add to existing practice relating to travel for transformation (Clark \& Cresswell, 2010; Guba \& Lincoln, 2003). Moreover, this project was an initial attempt to explore a study-abroad program through both transformative learning theory and critical race theory lenses. Therefore, the purpose of this project was to describe what elements of the transformative learning process students who participated in a month-long, studyabroad program in Spain experienced. In addition, I explored what the experience of traveling to an "other" place and experiencing "otherness" had on transformative learning (Johnson-Bailey \& Alfred, 2006; Morgan, 2010). Furthermore, I examined transformative learning through the critical race theory lens of Whiteness to analyze what role racial and cultural privilege played in travel for transformation.

\section{Research Questions}

In order to achieve the purpose stated above, the research in this project was guided by the following questions:

1. To what extent did eight students experience the elements of transformative learning during a month-long, study-abroad experience in Spain?

2. What role did encounters with cultural and linguistic "otherness" play in the transformative learning (or lack thereof) of each participant?

3. What was each student's racial, economic, gender, and cultural identity? How did each student's identity impact her or his worldview and transformations (or lack thereof) during the month-long, study-abroad experience? 
As I looked through the lens of these research questions, I identified overarching patterns or themes that emerged from comparing the individual findings across groups of participants. Furthermore, I compared and contrasted these patterns with studies cited in current academic literature regarding critical social TLT, CRT, and travel for transformation. In addition, I focused on how socio-cultural variables (race, gender, cultural identity, class, and sexual orientation) increased (or decreased) participants' opportunities for critical social transformative learning. It is important to note that the purpose of this dissertation was not generalizability, which would be impossible with the sample size $(N=8)$ and purposive sampling method utilized to select students for this dissertation (Clark \& Cresswell, 2010; Merriam \& Kim, 2012). Rather, the purpose of this project was to obtain deep, rich, and complex understandings that reflected the perspective of the students involved in the study-abroad program in order to delineate a grounded theory as to how adults learn and transform toward more permeable worldviews (Gall, Gall, \& Borg, 2007; O'Sullivan, 2012). Thus, I have analyzed the data through the lenses of the research questions, relevant literature, and the data collected in the study to provide the most plausible explanations of why students experienced varying levels and types of transformation (Marshall \& Rossman, 2011). This generation of patterns and themes is at the heart of grounded theory (Glaser, 2012). Because I utilized constructivist grounded theory (Charmaz, 2003), seeing participants as co-participative storiers (Pritchard, Morgan, \& Ateljevic, 2011), I will continue to highlight their voices and narratives in this chapter.

In order to discuss the outcomes related to the research questions, I will elaborate 
on cross-participant patterns and themes that emerged from data collected and analyzed

for this study. Next, I will provide conclusions that emerged from analyzing the findings of this study. Following, I will list limitations that may have impacted the transferability of the conclusions, citing countermeasures I took to diminish the impact of those limitations, where possible. Afterwards, I will express implications of this study in the context of existing research and suggest future possible research to answer new questions that arose during the analytical process. Lastly, I will summarize the research.

\section{Cross-Participant Patterns or Themes}

I will discuss cross-participant patterns or themes under the subheadings of the research questions to which they correspond in the following sections.

To what extent did eight students experience the elements of transformative learning during a month-long, study-abroad experience in Spain? One pattern I observed during the study-abroad program was the formulation of themes for each of the weeks of the study-abroad program that intersected among multiple participants. These themes were noticed and supported by the participants themselves as we discussed their process of cultural immersion during individual and focus-group interviews. The first week had a theme of culture or language shock for most of the participants (with Tess, Kynzee, and Tim calling it culture "surprise"). The second week, I observed (and the participants verified) was a week in which the students became immersed in the culture of their host family, with both positive and negative outcomes. During the third week, students became accustomed to the rhythm, routines, and culture of Spain, ending the week with the festivals. This was the week I started to hear more positive narratives 
about Spanish culture. Finally, the fourth week was a week of reflection of the impact of the study-abroad program and lessons learned that the students hoped they would carry home with them.

Week one: Culture shock/surprise. I received far more descriptions of culture shock during the entrance interview than in all other interviews (semi-formal or informal) combined. By the beginning of the second week, most of the students changed their narratives from culture shock to cultural surprises. By the exit interview, almost all culture shock had dissipated, with an exception of the bullfight for several of the participants. By the member-check interview, participants were more likely to romanticize their experience than express shock. Repeated culture shocks that appeared cross participants are discussed below.

Besitos, and public displays of affection. All of the participants except Tess and Tim commented on besitos and public displays of affection being a shock when they arrived in Spain during the entrance interview (some also used the term "lack of personal space"). Kristene and Kylie continued to struggle with besitos and the "touchy-feely nature" of the Spanish, but I believe that their continued struggle had more to do with their nature (both of them stated that they did not enjoy physical touch) rather than a continuation of culture shock. In contrast, Jennifer, Becky, and Anne felt like they adapted to the besitos and the close-proximity (compared to many Americans) with which the Spanish carried on conversations. By the entrance interview (on the first Friday of the study-abroad program), Kynzee had come to enjoy the physically affectionate nature of the Spanish, even though it initially surprised her. In contrast to the 
rest of the group, Tim and Tess appeared to be comfortable with these traditions immediately.

Nudity. Nudity (topless women on the beach, art, statues) was a cross-participant theme of culture shock among Kristene, Jennifer, Anne, Becky, and Kylie. Tim, Tess, and Kynzee explained that they expected to see nudity because they had done previous reading about it in Europe. I wrote an analytic memo immediately following the member-check interviews explaining my observation of their opinions of nudity as a metaphor for many of the students' transformative learning. I wrote:

Nudity seemed to be a symbol for transformation of "opening up." Originally, the students found it shocking. It was talked about in the interviews and I overheard them discussing nudity frequently during the first week of the study-abroad program. However, five months later, in retrospect, they did not find it shocking at all. However, a theme emerged wherein they found it acceptable over there, but would be greatly shocked if it happened in the United States (Kristene, Jennifer, Anne, Becky, and Kylie all shared similar thoughts). I wonder if that is a good analogy for a lot of the transformations that did not continue once the participants returned to the United States.... It is okay for them [over there]. Many of the participants experienced objective reframing about "them over there" but not [subjective reframing] about "us over here." What did result, however, by going to another culture, was a repeated theme among these five participants was an increased measure of tolerance, an understanding that things are different 
everywhere, and that (to them) is okay.... I believe this tolerance may eventually lead to subjective reframing.

Likewise, Tisdell (2012) suggests that cognitive dissonance often takes years, even decades, to turn into transformative learning. I maintain that a growing sense of tolerance is a positive transformative outcome even if socio-cultural behavior does not observably change immediately.

Language. Although I highlighted this shock in Jennifer's narrative, I wrote in my field notes that most of the students (except for Tess and Kristene) looked like "deer in the headlights" during the first couple of days of class. However, by the end of the first week of classes, I wrote that the LU professors were speaking at a natural cadence and that the students appeared to comprehend much better (observed by answering questions appropriately, laughing at jokes the professors told, or turning to the appropriate pages in textbooks).

Ironically, the more comfortable the participants became listening in Spanish, the more they began to speak English when they were not compelled to speak in Spanish. I will discuss this further in the section on linguistic and cultural "otherness" below.

Most "shock" did not last. I observed that myriad other culture shocks dissipated from the students' conversations almost as quickly as the students could explain them. For example, Kristene and Jennifer initially struggled with the need to take shorter showers (water and butane are much more expensive in Spain than in the United States). Additionally, Jennifer and Kylie found the food conservation practices of the Spanish repulsive. Moreover, Kristene, Jennifer, Anne, Kylie, and Kynzee experienced difficulty 
navigating foreign airports. In addition, Jennifer and Becky did not like the custom of multiple diners eating from a shared plate. Also, Becky, Jennifer, and Kristene commented on feeling uncomfortable due to the "classy" dress of Spanish women. Plus, Jennifer and Kylie felt that the classes were too difficult. Lastly, Anne, Becky, and Jennifer struggled getting used to the "laid back" nature of the Spanish schedule (later wake-up and school start times, people not showing up at meeting times, and slower pace of public transport). However, what unified all of these shocks is that they were short lived. The participants mentioned them in the entrance interview and then adjusted quickly during the second week of classes.

Week two: Immersion in family culture. During the second week of class I overheard or observed many indications that the students were transitioning from culture shock to a feeling of immersion with their host family. While Becky and Kynzee were extreme examples of family inclusion throughout the study-abroad program, all of the students shared narratives about activities they were doing with host families this week (new beaches, restaurants, going to other cities, barbeques, ice cream parlors, and family parties). Additionally, all of the students manifest signs of growing bonds with and care for their host families.

Please call my host "mom." For example, on Monday of the second week I reminded the participants that we were going to conduct a focus-group interview. Jennifer, Anne, and Kylie told me that they had forgotten to mention this to their host "moms" and were worried that their "moms" would make lunch for them and the participants would not be there. They asked if I could have the LU housing coordinator 
call and inform their host "moms" that they would be home an hour late. I wrote in the field notes, "I took this care and respect of not wanting to come home late for la comida [the biggest meal of the day] to be an indicator of caring rather than [perceiving the host families as] 'the help' that I observed previously."

How do I make plans with my host family? During this week Kynzee and Becky asked me for a grammar lesson of how to make plans in the future tense. They felt that they had disappointed their host "moms" because they did not understand the difference between when they were discussing ideas about what to do and when they were making actual plans. They did not want to offend their host "moms."

Kristene became a "sister" to her younger host siblings. Moreover, Kristene's journal was full of activities that she and her host siblings did during the second week. They went out to the beach and to restaurants. In addition, Kristene helped her younger host siblings with their homework. She wrote about assisting them with homework with pride. Kristene also spent about half of her time in Madrid at the end of the second week looking for souvenirs to bring home to her host family.

"Like family." Tess, Kristene, and Tim all commented that one of their shocks was how similar their host families were to their families in the United States. This led them all to seek a more positive relationship with their families.

Negative impacts of immersion in the host family. Not all of the immersion was positive, however. Kylie was invited to a barbeque where she felt like her host "mom" excluded her and made fun of her toward the end of the second week. During the second week, Kristene's host "brother" made a comment about her being too "fat" to fit into 
Spanish clothes (and then it became a "continuous joke"). Furthermore, this week Anne was reprimanded for not following host family laundry procedures. Anne also explained that her host family and fellow exchange student did not talk to her when she was in the house, which made her sad. In addition, Tim — who felt like his host family was a likeness of his United States family—expressed a concern the second week that he did "not fit in" and did "not live up to the expectations" of his host family. I posit that even the negative experiences showed that the students started to sincerely care what their host families thought about them. They expressed desires to show greater respect for, and be respected by, their host families.

Week three: Immersion in Culture outside of the host family. A repeated theme of immersion in the host culture started to emerge during the third week of classes. This theme was manifest in the participants' desire to know other students, know more about the Spanish culture, their growing activities apart from the host families, and from the festivals.

Positive cultural surprises. Multiple students (Tess, Tim, Becky, Kynzee, Kristene, and Jennifer) explained how much they enjoyed walking more than they did in the United States. During Ana's class, Tim expressed (in a disgusted tone) that many people in the United States take cars to go a couple of blocks. Moreover, students' (Jennifer, Tim, and Kynzee) questions, debates, and interest increased about the politics and culture of Spain during the third week (noted by the numbers of comments they made about this topic in LU classes). 
Extending travel. The students also began planning events as a PSU group to venture beyond the routines of their host families during the third week. For example, all of the students except Kristene and Anne ventured beyond the city limits of Levante and took a ferry out to an island in the Mediterranean. Kristene did not go because she was on a self-guided trip to Madrid. Additionally, I overheard the participants discussing plans to go to bars, nightclubs, and restaurants with each other. I wrote an analytic memo suggesting that all of these events were indicators that the students appeared to be extending their cultural immersion beyond the host family to the host culture.

Festivals. All of the students had overwhelming positive experiences during the festivals. It was evident (from the students' and host family statements and from my observations) that the host families aspired to showcase the festivals. Additionally, Juan and Ana discussed the festivities in their classes and Dr. Green invited them to take full advantage of every opportunity to celebrate with the people of Levante. From reviewing the data on their experiences during the five-day-long celebration, I maintain that Becky's statement about her host mom "I just try to keep up" was experienced by all of the participants. In fact, the only negative accounts I heard from the festivals was Kynzee's disappointment in Paco for using hashish and several students' (Kristene, Kylie, and Jennifer) disappointment that they stayed up to see the sunrise, but it was too hazy to fully appreciate it. However, while all of the participants enjoyed the festivals, several underwent major transitions in their attitudes toward the host culture and the LU study-abroad program during the five-day celebration. 
Jennifer called the festivals the "turning point" for her study-abroad experience, which I think is a great title for the experiences of Anne, Jennifer, and Kristene as well. For example, Anne, who stated, "I can't wait to get the fuck out of here" the day before the festivals started, told me in the exit interview "I don't want to leave" and "I hope to come back to study for a year." Moreover, Jennifer, who right before the festivals unloaded a list of complaints about the LU study-broad program explained all of the things she loved about the culture and said she wished she would have sought cultural immersion sooner. Furthermore, Kristene, complained that the bus driver got a 45minute break every two hours on the trip to Granada. However, after the festivals, she stated that she wished that Americans could learn from the Spaniards to not work so hard and to enjoy life more.

For all three participants, this change in narrative came from a deep sense of inclusion from someone during the festivals. For Anne, Paco and Tess invited her to partake in many of the activities they were experiencing. I wondered in an analytic memo if Tess saw another member of the group who had been marginalized by the travel group in Anne (even though I personally observed that Anne self-selected not to participate in many activities and had previously talked about Tess' neighborhood in the United States with deficit viewpoints). However, during the exit interview, Anne spoke often of the gratitude she felt for Tess' inclusion and cited Tess as a contributing factor to her turnaround. Furthermore, Jennifer enjoyed the festivals through the inclusion of her host "sister," Magdalena. Lastly, Kristene's inclusion from and affection for her host 
family played an important role in her transformation, leading her to say in the exit interview, "I'm pretty sure I am not going to want to leave."

Bullfight. The bullfight resulted in the most diverse and nuanced responses from the participants out of all of the cultural excursions. Kristene, Jennifer, and Anne "hated" the bullfight. Kristene “didn't think it was necessary to be killing all of those bulls for entertainment." In her journal, she wrote, "I almost puked watching that." Likewise, Jennifer felt the bullfight was "even more horrific than" she anticipated. She found herself focusing on the gory parts of the event, which made her "hate it even more." Moreover, Anne was very vocal about her "distaste" for the event and stayed through the whole event to cheer for the bulls.

Other participants saw both positive and negative aspects to the bullfight. Becky "felt bad" for the bulls, but understood it was part of the culture she was trying to study. She and Kynzee were dressed up in traditional Levantino attire for the bullfight. Both of them appreciated the cultural aspects. Becky liked "the music and the atmosphere... of the historical coliseum." She also added, "I don't like this, but it is the culture." Additionally, Kynzee felt "she was prepared" by the LU professors, Dr. Green, and her host "mom." She felt that the students who complained refused to look at the bullfight from the Spanish perspective and stated that her classmates were being "ethnocentric." Tim and Tess appeared to be enjoying themselves at the bullfight. Although both of them "found the killing hard to watch," they enjoyed the sense of community. Tess could really "feel the community and culture," which helped her "connect with being Spanish." Both maintained that despite appreciating the sense of community, they hoped to never see 
another bullfight. Finally, Kylie felt the bulls lived "a much better life than they do in the United States" and enjoyed the spectacle of the toreador.

When I asked the participants if there was a cultural equivalent to bullfighting in the United States, answers included dog fighting (Jennifer), NASCAR races (Anne), Rodeo (Becky), and the "annihilation of Native American tribes" (Tim).

Week four: Reflection and romanticization. The last week of the study-abroad program, students reflected upon the lessons they learned, things they wished they had done differently, and especially, expressed fond memories. Jennifer felt that week four should be titled "return to reality" because she had to start preparing to return to school and tried to get everything done before she left that she wanted to do. Moreover, students were prone to over-romanticization of their time in Spain. Ironically, the students who had most vocally complained during weeks one and two were the ones who said, "I haven't done everything I came for," (Jennifer) “I wish I would've done more," (Anne), and "I don't ever want to leave" (Kristene). Additionally, Anne and Tess informed me that they wanted to return to Spain to teach English for a year following graduation. Most importantly, the participants were prone to explain what they had learned and how they had changed during the study-abroad program, which will be described below.

Transformations. Although the transformations varied greatly among the participants, all overarching, cross-participant themes relate to the second and third research questions. Issues relating to cultural and linguistic "otherness" (or inclusion) and will be addressed in the following section. Moreover, cross-participant themes that arose relating to students' identities will be outlined in the subsequent section. 
What role did encounters with cultural and linguistic "otherness" play in the transformative learning (or lack thereof) of each participant? I will address crossparticipant patterns and themes related to the impact cultural and linguistic "otherness" had on the transformative learning of each participant. Following, I will explain the positive role cultural inclusion played in the transformative learning of several of the participants, which was a departure from the literature reviewed for this dissertation.

Cultural “otherness.” Several of the students (Kristene, Jennifer, Anne, Becky, and Kylie) disliked "sounding American" (Jennifer) or "looking like a tourist" (Kristene). Jennifer stated, "I think the hardest thing to adjust to is walking around every day knowing everyone knows you're different. And everyone is constantly judging us.” In addition, Anne also feared that she "looked like a tourist" and pointed out that her red hair and light eyes made it obvious that she was not from Spain. Also, Kylie commented several times that she did not like bringing attention to herself, yet people automatically knew she was American by her accent and dress.

These experiences with "otherness" are similar to the daily indignities people of color face in the United States: worrying that people will judge their whole race, class, group based on how they act or what they wear (Bell, 2009; Ladson-Billings, 2009; Solorzano \& Yosso, 2009). Yet, it was evident from these and other incidences that this feeling of "otherness" was not something to which these students were accustomed. The participants made no subjective leap to how racially, linguistically, and economically marginalized individuals are culturally "othered" in the United States and how their own epistemology, rooted in unearned privilege, might reinforce that marginalization. 
Nevertheless, I am hopeful that the experience in cultural "otherness" created sufficient cognitive dissonance to initiate "a progressive series of transformations in related points of view that culminate in ... transformation" (Mezirow, 2012, p. 86). My hope rests in Tisdell's (2012) caution against educators constantly seeking the "peak experiences" (p. 31) of epochal change. She argues that many incremental changes, over time, can lead to equally as poignant of a transformation, but the transformation may happen much later in the participants' lives. Furthermore, Tisdell posits that trying to force transformation on an unwilling participant may elicit an anti-transformational response (see also Mezirow, 2012).

Linguistic "otherness." In contrast to cultural "otherness," perceived linguistic "otherness" created opportunities for Kristene, Jennifer, Kylie, and Becky to subjectively reframe their discriminatory attitudes about speakers of other languages. Consequently, each of them experienced the desire to reduce linguistic oppression for English learners upon their return to the United States.

Becky was the most proactive out of the group upon her return, seeking licensure for a teaching endorsement for English learners. She wanted to obtain a master's degree in English for speakers of other language and to become a specialist to assist little children who were English learners after graduation. Moreover, Becky stated it was important to her to "incorporate both cultures" as she taught elementary students English. Likewise, Kristene (also an elementary education major) described in her journal a linguistic epiphany that happened to her while she was helping her host "sister" with English. She realized that the host sister would probably never get English pronunciation 
perfectly, just like she would never get Spanish pronunciation perfectly. Kristene felt that this inspired her to find ways to help English learners in classrooms when she became a teacher. Also, Jennifer explained to me her difficulty in understanding Spanish and feeling "foolish" to try to meet Spanish young adults had made her much more empathetic to English learners in the United States. Next, Jennifer went on to explain that previous to the study-abroad program, she "did not have a lot of patience" with English learners. However, after experiencing linguistic "otherness," Jennifer felt like she looked for opportunities to assist English learners. Kylie also had several stories in which she had advocated for English learners upon her return home.

I observed that experiencing linguistic marginalization led to these participants' transformation toward linguistic inclusion (Johnson-Bailey \& Alfred, 2006). The participants' socio-cultural transformation to be more inclusive of English learners in the United States aligns with Foster's (1997) theory that learning another language can be transformative. Foster (1997) asserts that learning a second language is linked to the destabilizing experience of Mezirow's (2000) disorienting dilemma. She argues that when learning another language, there is typically a mild degree of anxiety or vulnerability associated with learning another language. Consequently, this vulnerability sets up an excellent situation for transformative learning to occur. In addition, Goulah (2007) found that students who learned another language while immersed in that culture developed critical socio-cultural attitudes about their home culture. These students' transformation toward linguistic inclusion was still impacting their attitudes and actions to improve society for "others" at the time of the member-check interview several months 
following the study-abroad program. Scholars maintain that once a person has had a transformative change she or he will never go back to the old way of seeing the world (Mezirow, 2012; O'Sullivan 1999, 2012). Therefore, I posit that linguistic "otherness" had, and should continue to have, a lasting change on the way these participants see the world and act in it.

Kynzee must also be addressed in this section on linguistic "otherness." Kynzee's efforts to speak Spanish, even when she did not have to, endeared her to members of the host culture, rather than made her "othered" by them. This vulnerability not only opened her to a state of permeability, it appeared to open members of the host community to want to share their knowledge with her, as well (Goulah, 2007). Kynzee's willingness to be seen as a representative member of the host culture (Ross, 2010) overcame the linguistic "otherness" that often is experienced by study-abroad students who are immersed in a culture whose language is not their heritage language (Foster, 1997).

\section{Inclusion had more impact than "otherness" for transforming opinions about}

culture. Kynzee's inclusion, due to effort rather than linguistic proficiency, was one of the factors that made the conversation with her host "brother" about gun culture possible. I believe that her positive relationship with him led to an opportunity where he could be critical about United States gun culture. Consequently, Kynzee's permeability and critical reflection led to her epochal transformation and subsequent action regarding the amount of and ease of access to guns in the United States. Kynzee's radical inclusion with her host family (as well as other students' sense of inclusion with their host families) 
led me to ponder the role cultural or linguistic inclusion (as opposed to "otherness") played in the transformative learning of the study-abroad participants.

Many examples of cultural inclusion by a member of the host culture leading to subjective reframing exist among the participants. For example, Kristene's desire to teach English learners in the United States was grounded in the cultural inclusion, not "otherness," she felt with her host siblings. Likewise, Becky gave linguistic "otherness" as part of the reason she was seeking a master's degree in teaching English to speakers of other languages. However, the more critical social outcome of desiring to be culturally responsive and value the funds of knowledge of her students' families was credited to the inclusion she felt from her host family. In addition, Jennifer was extremely critical of Spain, its people, and the study-abroad program until she spent a festival weekend with her host "sister," Magdalena. Subsequent to that experience Jennifer's attitude about Spain and its people dramatically changed. Moreover, Anne's inclusion by Tess and Paco during the festival weekend led to her to desire to return to Spain to teach English for a year. In contrast, Kylie's sense of "othering" by her host "mom" led Kylie to become impermeable to Spanish culture. However, the linguistic "niceness" (a form of inclusion) by individuals who spoke English instilled in her a desire to repeat this "niceness" to linguistic "others" in the United States. Similarly, Tess's lifelong "otherness" of insider/outsider role to both Latinas/os and White individuals served as a sharp contrast to the radical sense of inclusion she felt as she found "her country" and "her people." 
I postulate that it was the students' inclusion, more than their sense of "otherness," that impacted their development of cultural awareness. This hypothesis aligns with Goulah (2007), who found that students who learned another language while immersed in that culture developed critical socio-cultural attitudes about their home culture. However, literature regarding cultural inclusion is under-conceptualized and under-theorized at the time of this dissertation. Consequently, I will recommend further studies to analyze the impact of cultural and linguistic inclusion on critical social transformative learning in the recommendations for future study section below.

What was each student's racial, economic, gender, and cultural identity? How did each student's identity impact her or his worldview and transformations (or lack thereof) during the month-long, study-abroad experience? Throughout this section I will describe cross-participant themes that emerged from this study indicating how students' identities regarding class, politics, future professions, and racial/cultural heritage impacted their worldviews and transformative learning.

Class privilege. Class privilege had an observable impact on the study-abroad participants' worldviews and transformative learning. Consequently, I decided to order the participants in the Findings chapter from highest socio-economic privilege to lowest (based on self-identity and my analysis of the students' comments). Kristene, Jennifer, and Anne self-identified as upper-middle class. Becky, Tim, and Kylie self-identified as middle class (although Kylie was from an affluent neighborhood, her father's long-term unemployment led her to self-identify as "lower-middle class." However, many of her attitudes were influenced by the affluent environment in which she was raised). Kynzee 
never self-identified. However, her use of work-study, Pell grants, and multiple other grants designed for working class students, the median income of her town, and her family's communal effort to raise enough money for the study-abroad program led me to postulate that she came from a lower-middle class background. Finally, Tess explained that she grew up in poverty.

As I analyzed the data, I observed that upper-middle class students experienced epochal transformations that were personal transformations rather than critical social transformations. For example, Kristene's epochal transformation was a feeling of greater independence. Similarly, Jennifer was able to "break out of the bubble" of social comfort in the United States to experience another culture and language. Likewise, Anne came to understand, through critical reflection, that the abuse she had suffered from males was not her fault. This liberation from guilt led her to feel more confident as a student and person. Lastly, Kylie (she was middle to lower-middle class by family earnings at the time of the study-abroad program, but shared an upper-middle class epistemology permeated by the affluent community values in which she was raised) saw the studyabroad program as a "right of passage" and opportunity to experience new things.

All of these transformations were personally powerful and aligned with Mezirow's $(2000,2012)$ version of TLT in that the students became more autonomous. However, while the participants' subjective transformation was deeply engaging and important on a personal level, it did not appear to motivate them toward more justified frames of reference or action toward others. Accordingly, the most repeated critique of Mezirow's $(2000,2012)$ TLT is that it is overly focused on individual transformation and 
does not focus directly on social transformation (Tisdell, 2012). Critical social TLT, however, goes beyond merely empowering participants to change their lives for the better and insists that participants act to improve society as well (Merriam \& Kim, 2012). Furthermore, from a CRT perspective, because society privileges White, upper-middle class individuals (Leonardo, 2009), social power structures are designed to allow transformations of this type to be merely personal, while individuals who lack privilege must develop an understanding of unjust social structures merely to navigate an unjust world (Johnson-Bailey \& Alfred, 2006). For the upper-middle class participants, their epochal transformation was a "pedagogy of the privileged," rather than a vehicle for socio-cultural transformation (Curry-Stevens, 2007).

Shift to the political "left." Many of the participants voiced epistemological shifts toward the political "left." Fascinatingly, this happened regardless of critical social transformational outcomes at the time of the study. For example, Kristene stated that the political discussions in both classes and her observations of gender and LGBTQ+ freedoms in Spain made her "even more liberal than [she] already was." Likewise, Jennifer experienced a deepening of socio-cultural consciousness through an awareness of "others" in Spanish society. Moreover, Kynzee developed a critique of gun culture in the United States. Additionally, Becky, Jennifer, Kristene, and Tess felt like life in the United States was too materialistic and wanted to live more "day by day." Lastly, Tim, Kynzee, and Tess loved the sense of community they witnessed in the bullfights and festivals. Some of these students experienced critical social subjective reframing (Tess, Kynzee, and Becky), while others did not state changes in critical social shifts in 
behavior. Nevertheless, there was an apparent shift in awareness, even if the awareness had not led to social action at the time of the study-abroad program.

In the section above, I critiqued the upper-middle class students' increase in autonomy absent of a stated desire effect social change. Throughout data analysis, I questioned how the participants' shift toward a more "liberal" epistemology did not activate a desire to positively impact society. To try to understand this phenomenon, I reviewed Curry-Stevens' (2007) article on travel for transformation as a "pedagogy of the privileged." She maintains that participants need space and time to connect to greater social causes. Additionally, she argues that study-abroad participants come from varied political and socio-cultural backgrounds. Likewise, although the LU study-abroad students varied in socio-economic, racial, and even political epistemologies, many of the participants became more aware of feminism, LGBTQ+ issues, impacts of war and capitalism, and myriad other marginalization issues within Spanish society through the LU classes. As I read Curry-Stevens' article again, I realized that although many of the students had not stated a desire to enact social change at that time, I was reminded of Tisdell's (2012) caution against trying to push students too hard for immediate transformation. In addition, I also came across this analytic memo I wrote after the last day of classes in my field notes as I pondered the role political epistemology played in the students' experiences:

I ... believe that the students have gained more in a ... permeable/open-minded worldview than they have in Spanish [proficiency].... I was intrigued at their capacity to debate [with nuance] politics, religiosity, and other complex subjects 
during ... Juan's [and Ana's] class[es]. They went beyond the [Democrat vs. Republican false binary] that we have in the United States. [In contrast,] they showed ... deep thinking in the way they talked about Catalunya and Pais Vasco wanting to separate [from Spain.] They also discussed the political correctness [regarding] religiosity in the United States versus the [religious privilege embedded in] the grand religious festivals in Spain. It was not the [arguments themselves] that impressed me, but their willingness to consider new ideas. Their facial expressions showed ... "That's interesting, I've never thought of that before.” They came up with ideas that I could tell came from actually thinking about it rather than regurgitating [talking points] they had seen on news shows.

From a critical social stance, this willingness to consider new ideas (especially ones related to marginalization) may be seen as a socio-cultural awakening. Likewise, CRT scholars contend that socio-cultural awareness of marginalization leads individuals to validation and affirmation, which, in turn, hopefully leads to social action (DelgadoGaitan, 1993; Ladson-Billings \& Donner, 2005; Smiley \& West, 2012). Consequently, I leave this research with a hope of "not yet" when it comes to critical social transformations for the majority of the participants. I recognize that the experiences they underwent may continue to impact them for years, or even decades, to come (Merriam, 2004; Tisdell, 2012).

\section{Conclusions}

Upon returning home from Spain, I immersed myself in the data for several months, spending hundreds of hours listening/re-listening to interviews, reading/re- 
reading field notes, going over observations, and looking at all of the photographs. Marshall and Rossman (2011) maintain that this process "forces the researcher to become intimate with the material" (p. 210). I coded and categorized the findings, marked up initial themes, and wrote the findings. Throughout this cyclic process of reviewing and re-visiting data, I enlisted the assistance of two critical scholarly colleagues to search for alternative understandings.

Clark and Cresswell (2010) maintain that after codes have turned into patterns, and patterns into overarching themes by analyzing data, it becomes essential to start to generate and describe a grounded theory. Therefore, I describe conclusions from the constructive grounded theory analysis that emerged from the co-creation of themes that emerged from this study below (Charmaz, 2003).

\section{Racial privilege impacted subjective reframing and critical social critical}

reflection. The findings of this study reinforce Johnson-Bailey and Alfred's (2006) theory that marginalization leads to critical reflection and subjective reframing. However, marginalization alone did not result in critical social outcomes. For example, both Kylie and Anne experienced perceived cultural "othering" by their host families and disliked the feeling of being in the streets where everyone could ascertain they were not members of the dominant society. However, this marginalization led to stereotyping of the host culture, rather than an understanding of the dominant positionality their racial and class privilege afforded them in the United States.

In contrast, socio-cultural variables of racial identity and class (which is at the heart of Johnson-Bailey and Alfred's article) and the lifelong experiencing with 
"otherness" had a greater impact on the critical social reflection of both Kynzee and Tess. For example, Kynzee and Tess's sense of purpose rooted in their desire to connect to their racial and cultural identity led to a sense of inclusion that directed them both to reflect on social issues within the United States (Kynzee about gun culture and Tess about her insider/outsider role in both Latina/o culture and White culture).

Racism is normal, Whiteness is normalized. Overwhelmingly, however, for the students who identified as White, the normalization of Whiteness within United States society made it difficult for them to see the embedded codes, customs, and conventions in governmental, institutional, and corporate systems that promote White supremacy within their own culture (Taylor, 2009). This group of students could see the flaws in being "othered" by Spanish society, but the racial privilege they experienced in the United States was invisible to them (Brah \& Phoenix, 2009; Brookfield, 2012). The normalization of Whiteness was such a powerful social construct that even the participants who self-identified as political liberals (Tim, Kristene, and Jennifer) were unable to realize that the marginalization they experienced in Spain was similar to the daily indignities people of color (and other minoritized groups) face in the United States (Delgado, 2009a; Ladson-Billings, 2009).

Tess and interest convergence. In addition to their own racial privilege being invisible to them, many of the White students (Becky is an exception) re-created and reified racial privilege through interest convergence concerning Tess (Parker \& Lynn, 2009). As discussed in Tess's findings, the students often took advantage of her cultural capital (access to the "authentic" student host culture) when it worked to their benefit, but 
had little observed or stated desire to give back to Tess or the host community (Delgado, 2009a; Gillborn, 2009; Ladson-Billings, 2009).

Race is a social construct. CRT scholars maintain race is a social, not biological construct, that changes with the needs of the dominant culture (Delgado \& Stefancic, 2001; Parker \& Lynn, 2009; Solórzano \& Yosso, 2009). One of the surprising outcomes of this study for me as a researcher was being forced to confront the Black vs. White binary that is often the dominant narrative in CRT scholarship (Ladson-Billings, 2009). However, Solórzano and Yosso (2009) argue that it is important to shift the discussion of race from a White vs. Black narrative to a more complex discussion with many faces, voices, and experiences. Tess and Kynzee pushed my boundaries of understanding the societal borderlands of individuals who lie at intersections of marginalized groups (Crenshaw, 2009). Both Tess and Kynzee seemed to daily navigate among contradicting aspects of self, creating a sense of both inclusion and "othering" simultaneously (Johnson-Bailey, 2012). While I observed Kynzee was able to navigate between the worlds of "Black" and "White," Tess was an insider and an outsider simultaneously in whatever community she positioned herself within the United States. Moreover, Kynzee seemed content with the celebration of and recognition that she was "only one-quarter Hispanic," while Tess discussed not fully being at "at home" until studying in Spain. Within the United States, Latinas/os saw Tess as not "fully" or a "real" Latina/o, while Whites marginalized her for her linguistic, cultural, and class identity.

Meeting these two participants complicated my understanding of racial identity and heritage. Especially within the Latina/o community, skin color does not necessarily 
represent cultural identity (Anzaldúa, 1990). More than ever before, I came to understand that race is a social (identity), not biological (skin color), construct that changes with the needs of the dominant culture (Delgado \& Stefancic, 2001). Even though Kynzee and Tess looked White (and most likely received unearned societal privileges based on that social construction), they lived in a borderland of intersecting marginalizations/privileges. Kynzee acknowledged other than her last name (a very common Spanish last name), no one would ever assume she was Latina (even though she identified as such). She recognized the unearned benefits that accompanied her social construct of Whiteness (from others) and acknowledged it in all semi-formal interviews. In fact, she was the only socially constructed (by others) "White" person who discussed racial privilege with me, even though she self-identified as a Latina. Tess, on the other hand, lived in a constant insider/outsider borderland of intersecting race, linguistic, and class marginalizations. She was not "at home" anywhere in the United States, but found "her people" in Spain.

Although many prefer to categorize identities into single social mechanisms, intersections of marginalizations (or intersections between privilege and marginalization) provide powerful rich new categories that need to be studied and understood (Crenshaw, 2009). Indeed, Anzaldúa maintained, "Necesitamos teorías ...that cross borders, that blur boundaries.... We are articulating new positions in the "in-between," (Anzaldúa, 1990, pp. xxv-xxvi). However, few research paradigms (especially within TLT literature) discuss, celebrate, or reveal the exciting borderlands in participants' lives (Taylor \& Snyder, 2012). Moreover, because dominant Euro-American culture is extremely 
competitive, rather than cooperative, few teachers, students, and researchers feel comfortable investigating narratives outside of "us vs. them" binaries (Brookfield, 2012; O'Sullivan, 2012). This silence reifies dominant social meta-narratives and limits our capacity to understand how humans construct knowledge from their rich, but complex, daily-lived experiences (hooks, 1994; Johnson-Bailey, 2012).

\section{Male privilege may have impacted Tim's lack of stated or observed}

transformation. Brookfield (2012) argues it is almost impossible for individuals in privileged positions to see the flaws in their own frame of reference (Brookfield, 1998). Therefore, it was possible for Tim to have liberal values while simultaneously escaping daily indignities that the females of this study encountered (Leonardo, 2009). Tim's male privilege was especially evident in his refusal to conform (or even notice) apparel customs (in sharp contrast many of the female participants' worry about how "classy" the Spanish women dressed). In addition, Tim also had a tendency to take over a discussion and talk like he was the authoritative voice on a subject (hooks, 1994). Despite "willingness," it appears that male privilege may have been a factor in his lack of perceived or observed transformation. Moreover, Tim wanted to experience (or "take") culture, but not become "like" them (Ross, 2010). O'Sullivan $(1999,2012)$ argues that the culture of "taking" from the earth and others is grounded in male privilege.

In contrast, I argue that Kynzee's critical social transformation about United States gun culture developed out of an absence of male privilege. In other words, her fear of rape or assault at gunpoint in the United States is what conditioned her to notice the difference in the way she felt "safe" living in Spain. In turn, this feeling of safety 
opened her to a critical social critique (and subsequent action) about United States gun culture.

"Sacrifice" to pay for study-abroad promoted a sense of purpose. Throughout the study-abroad program, students who sacrificed to pay for the study-abroad program themselves experienced a deeper sense of purpose. Morgan (2010) and Ross (2010) concluded in two different studies that a sense of purpose in travel was a repeated theme for students who experienced transformation. I posit this sacrifice to pay for the studyabroad program resulted in a sense of purpose for these students.

I use the word "sacrifice," rather than "paid" because there were inconsistencies using the word "paid." Tim, Tess, and Becky were the only students who paid for the study-abroad program with little or no financial help from others. In contrast, Kristene, Jennifer, and Anne's parents paid for most or all of their expenses. Among those who received financial support, the term "sacrifice" renders the pattern accurate. Kylie's insurance settlement from many years prior to the study-abroad program paid for her trip, requiring less stated sacrifice. Alternately, Kynzee (through work study, grants, and scholarships) and her family (she did not disclose how much or how they contributed financially) worked together to provide the opportunity for Kynzee to go to the studyabroad program. Kynzee repeatedly discussed the sacrifices of her family, others, and herself to be able to participate in the program.

I observed the less students sacrificed, the more likely they were to be dependent upon or complain about Dr. Green, the host families, and the LU staff (e.g. Jennifer's water situation). In contrast, the more students sacrificed, the more likely they were to 
identify solutions to their own problems and be content with the provisions, activities, and living arrangements of the LU study-abroad program. Ironically, the students who paid more for their own trip were the least likely to worry about or discuss the cost of the study-abroad program. Additionally, students who sacrificed less demanded that the study-abroad program help them "meet Spanish college students," while the students who sacrificed more, were more likely to seek out the host culture and take advantage of extra-curricular/extra-host-family opportunities to immerse in the culture (example Tess finding "authentic" college hangouts and Tim going to a winery at his own expense). Lastly, students who sacrificed more, were more likely to envision their role as houseguest and value the funds of knowledge of their host families, while participants who sacrificed less, tended to make comments about their host families as "the help," rather than a family whose culture was to be appreciated.

"Fit" between host family and student generated sense of inclusion or exclusion for participants. Kynzee and Becky participated in study-abroad programs that required staying with host families prior to the LU study-abroad program. Additionally, Kynzee and Becky both experienced immersion in the Spanish culture through a sense of radical inclusion they experienced with their host families. I have tried to tease out whether this was Becky and Kynzee's natures, their experience with previous study abroad, or the characteristics of the host families that created an atmosphere of radical cultural inclusion. Because it only happened for Kynzee and Becky, it is impossible to isolate which or what combination (if not all of these variables) led to the Kynzee's and Becky's transformations toward socio-cultural enlightenment. 
Nevertheless, by analyzing what they said, I posit that this radical inclusion was a positive feedback loop that became mutually beneficial between the participants and their host families. I theorize (and Becky's host "mom" and "dad" confirmed) that the inclusion was created equally by both the participants and their host families. Although Becky and Kynzee were the most extreme examples of this positive feedback loop of cultural inclusion, similar experiences occurred for Kristene and Jennifer. Jennifer, a skeptic of the host family system during the focus-group interview (halfway through the program), came to see the host family system as a "good thing" and she thought it "immersed [her] even more" than she would have had she lived in a dorm because it "forced" her to speak Spanish. Moreover, Jennifer's sense of inclusion from her host "sister" Magdalena augmented this feedback loop.

In contrast, there were examples when the feedback loop became negative thereby decreasing the perceived sense of inclusion, leading the participants to feel "othered." For example, according to the housing coordinator, Anne's host "mom" was the most inclusive, tolerant, and special-needs friendly (Anne was allergic to wheat) person the housing coordinator employed. Yet, Anne had a negative experience and felt excluded by her host family, even though the marginalization was simply her host "mom" asking her to follow house rules regarding laundry procedure (i.e. Anne stopped the positive feedback loop despite having an inclusive host "mom"). However, when I reviewed some of the comments and actions of Kylie's host "mom," it appears that the disposition of the host parent (at least through Kylie's perspective) also had a significant negative 
impact (therefore, in Kylie's case it is probable that the host "mom" stopped the positive feedback loop).

\section{Limitations}

There is no such thing as a perfect study, especially when conducting research with human participants (Clark \& Cresswell, 2010). Therefore, it was essential to decipher what kind of information I was trying to obtain, and design a study to answer the research questions (Marshall \& Rossman, 2011). Throughout this section, I will describe components of the research design that limited transferability of the findings to other study-abroad programs. In addition, I will explain issues that prevented me from obtaining full information from the participants.

Study-abroad program. The study-abroad program itself was a limitation because it was formed before I chose to research the participants' experiences. To protect his job as a professor and to protect the university from liability issues, Dr. Green could not change the study-abroad program from the prospectus that he created before I was invited to conduct research with the participants. Therefore, I had to investigate the transformative outcomes of the study-abroad program as it was created. However, one benefit of this limitation was that I added very few interventions - other than my presence, short interviews, and asking students to upload pictures to Flickr-which made me confident I complied with ethical codes of limiting interventions on participants (Vogt, Gardner, \& Haeffele, 2012). This further allowed me to be very respectful of the participants' time and energy. 
Several of the students vocalized their distaste that the LU classes only had PSU students. Jennifer stated during the focus-group interview:

I really thought we were going to be with other students. And I really wish that's how it would have been. I like Amy's system ... because she got to meet other people. I would have liked to have met other people but not just other people, but other Spanish people.... Integrate us with other people in the University, for God's sakes.

Nevertheless, when given opportunities by the LU staff or Dr. Green that were not compulsory, the students who complained about not meeting other students the most did not take advantage. Several examples include: an invite from the excursion coordinator to watch the daily festival fireworks show from very close, a free guided tour of the festival museum, and, Manolo's invitation during the Granada excursion to go out dancing. Typically, it was whomever Kynzee could convince to go with her that went to these activities (never Jennifer). The excursion coordinator expressed his disappointed in this group of study-abroad participants because they overwhelmingly choose to not participate in activities when he provided them (all of these examples were offered at no cost to the student).

In contrast, Kynzee and Tess often sought out—and found — extra-curricular cultural opportunities. In contrast, Amy—who had the program Jennifer and others claimed would be ideal—appeared (from Kylie's blog/interviews/my observations) to spend all of her free time with Kylie. From my observations, this was a participant personality, rather than a program deficit. 
From a linguistic and cultural "otherness" perspective, however, I posit that mixing the participants with students from other countries in Europe, or even better, 300400 level classes made for Spanish students, would have produced greater opportunities for critical social TLT outcomes. Students would have felt "stuck" like Foster (1997) described in not being able to even express what their struggles were due to lack of Spanish proficiency. However, from Dr. Green's/LU's perspective this system would have been complicated because the students would have had to attend for a whole semester. Furthermore, Spanish university semesters do not align with United States university semesters. Consequently, students would have had to miss two semesters at PSU for one semester of credit at LU. Nevertheless, from my observations, I believe mixing students in classes with other students from around the world would have been possible, because that is what Amy's program did (at least from Kylie's and Jennifer's descriptions). A program like this would have increased the critical social transformative opportunities because it would have increased the likelihood of cultural and linguistic "otherness."

Maleness. My maleness may have created a slower entry than I would have liked. Dr. Green made sure that I had almost immediate institutional entrance, yet I believe the seven female students would have made more complete disclosures had I also been female. During the first few days of the study-abroad program, I wrote about feeling disappointed in the speed of developing rapport with the (mostly) female participants. I wrote on the third day, "I feel like I am on a balancing act of not wanting to push to hard [to build rapport], but wanting to start collecting more data. I keep 
reminding myself that this is a marathon, not a sprint." However, upon analyzing the data, I observed most of these feelings dissolved in my field notes within a few days. During the bus ride to Granada at the end of the first week, I explained that I was going to do an interview with them, but I wasn't going to take too much time. One of the students (I couldn't tell which one) said, "Could you take more than 20-30 minutes, because I'm going to be bored!" During this cultural excursion, I wrote that I felt "I had attained entry." Examples I included were how students opened up to me about their boyfriends, how cute they thought Manolo (the Granada tour guide) was, and personal health issues. In addition, I noted that their speech codes changed from talking to a researcher to talking like they would with a fellow student. During the bus ride home from Granada I fell asleep. The students took joke pictures with me while I was sleeping and showed me the funny pictures they took when I awoke. I wrote in my field notes that I felt I had "managed entry."

Nevertheless, I feel that were I female, I may have obtained more complete disclosures from the female students, who were seven out of the eight participants. For example, in the exit interview, I asked Becky for her reactions to Ana's painting that challenged her perception of the cultural appropriateness of nudity. Becky turned red and would not discuss it. I could tell from her body language that she did not feel comfortable discussing this with a male (although I cannot definitively state whether she would have been comfortable discussing this topic with a female, either). Additionally, I would have felt comfortable asking if I could tag along on some of their adventures outside of class, were I a female. Examples include the several of the dancing or beach 
activities. I felt it was inappropriate to ask if I could accompany them because I was a thirty-seven-year-old male and they were twenty-something-year-old females.

Furthermore, I believe more students might have participated better in Flickr. One of the benefits of Flickr was the automatic download feature of whatever pictures one takes with her or his smartphone. However, none of the female participants felt comfortable turning this feature on as I helped them set it up (but Tim did). However, I believe the Flickr participation rate was a technology limitation more than a maleness limitation, and I will discuss it below.

Technology. Previous to this dissertation, I did a pilot study using Flickr with four graduate students on a five-day study-abroad experience in Mexico. Like in this study, I helped each of the participants sign up for an account and showed each of them how to access the privacy features and automatic upload features of Flickr from their smartphones. The participants enjoyed the group-sharing feature because they knew once someone took a picture of something, they were going to share it with the whole group. Therefore, the group of participants spent less time taking pictures and more time immersing themselves in the culture. In fact, the Mexico pilot-study group shared more pictures on Flickr from five days in Mexico than the Spain study-abroad group did in over a month.

The students in the Spain group (other than Kylie and Tim, who shared almost 90 percent of the photos) did not care for this data collection format. They felt uncomfortable setting the automatic upload feature and found the data bandwidth speeds at LU and in their host families' homes to be too slow to enjoy using a new website. 
They already were using Facebook or Instagram, which they felt did not require as much work (although I believe they were simply more familiar with those platforms). I believe students would have given me access to their Facebook or other social media by the beginning of the second week. Ironically, the reason I wanted to use Flickr is I felt it would be automatic and a minimal invasion to their privacy, while I think the students saw it as the opposite in both regards. I think because they already knew and used the other social media, they knew how to set the privacy settings. This is another occurrence where I feel my maleness may have provided a "creep" factor. I felt they were wondering, "Why does this thirty-seven-year-old man want to look at pictures of us?" In contrast, I observed them regularly posting on Facebook and Instagram from their smartphones.

If this were my group of students with whom I had a class beforehand, I may have set up a social media platform during the class, similar to the Goulah (2007) study that utilized photo and video narratives. Then, they could have been comfortable knowing how to manage data and privacy before the study-abroad program started. Additionally, I am capable with many modes of social media. I maintain it would have been better to simply ask them if they wanted to share their photos or videos with me on the social media platform of their choosing. I should have adjusted to their social media preferences rather than asking them to adjust to the social media platform that worked best for data analysis for me. Moreover, as a grad student trying to successfully defend a dissertation, I was afraid to vary too far from the accepted proposal. I feel that Facebook would have been a better data collection method for this particular group of participants. 
Nevertheless, Flickr, even though Tim and Kylie were the main uploaders, gave me insights into the extracurricular activities of all of the students (except for Kristene, to whom I promised I would not look at photos of her).

Age of participants. Merriam (2004) posits, from a neurological perspective, that humans' brains are not fully developed until they are in their thirties or forties. From this perspective, it may not be possible to experience "true" critical reflection until this point in a person's life. However, Mezirow (2004) writes a response paper to her and counter-argues that many of his students have had intense subjective reframing and critical reflection at ages much earlier than their thirties or forties. Furthermore, Goulah (2007) found that high school participants were capable of critical social subjective reframing and reflection. Furthermore, Johnson-Bailey and Alfred maintain that marginalization teaches marginalized students about social power structures before they even reach a college classroom.

Nevertheless, Tisdell (2012) states that transformative learning may happen much later than the transformative event. Consequently, many of the events of the studyabroad program may be a starting point that triggers future transformative learning, but it is impossible to know what the future will bring based on the timeline and time restraints of this study. Therefore, as I have analyzed the data and findings of this study, I have tried to Tisdell's (2012) "not yet" concept in the back of my mind.

Timeline. As I reviewed the data collected, I observed that one month is long enough to get a hint of what it would feel like to live in another culture (example, Tess). However, it is also short enough that it was possible for the students to stay in "tourist" 
mode (example, Kylie). For example, Anne (from her and others' accounts) spent large portions of time in her room video chatting with friends and family in the United States.

According to Dr. Green, study-abroad programs typically last between twelve days (Cordero \& Rodriguez, 2007) to one year (Brown, 2003). He could have chosen any length of time he desired. However, he labeled the twelve-day, study-abroad programs feel "super-touristic" and "voyeuristic" (in his opinion, students are positioned as takers of culture, rather than immersers with the culture in this short of a study). In contrast, he has observed that students have difficulty paying for any program that lasts longer than one month. The only exception he has found (and three of his students had participated at the time of the study-abroad program) is a program where students are paid to go teach English in Spain for a year. Thus, because the students are paid, they are able to offset the costs of traveling to Europe.

Dr. Green felt that the longer the trip the more likely the students are to have critical social transformative learning opportunities. Research on travel for transformation concurs with Dr. Green's observations. Cordero and Rodriguez (2007) found that not every student experienced personal or critical social transformation in their twelve-day study, whereas Brown (2003) found that every student experienced some form of transformative learning (and many of the transformations where critical social) in her one-year-long study. However, to Dr. Green's lament, more and more universities are moving to a twelve-day system because it costs less for students, is easier to arrange, the profit margins are higher for the university, and more professors are willing to participate. Therefore, in the exit interview (and then I verified in the member-check 
interview), I asked students what the "ideal time for a study-abroad program" would be. My conversation with Anne in her exit interview was the archetypical conversation about the timeline with the participants (except for Kynzee, Tess, and Tim). She replied, "I feel like a month was too short. I feel like it wasn't enough time to really learn the language. And it wasn't really enough time to get accustomed to your surroundings." I asked her how long would be an ideal length for a study-abroad program. Anne answered, "At least four months. Just because you're there, and you get to learn new things, and ... come up with a routine ... and you just don't have to adjust [to a new place and culture] ... super quickly." I inquired, "Would a pre-trip Anne go on a semester-long [studyabroad program]?" Anne laughed and responded, "Probably not."

Jennifer (who maintained a semester or six months was the ideal length of a program) was surer of her response to the question "Would pre-trip Jennifer have gone on a semester-long study-abroad program?" She answered in her exit interview:

I can absolutely, 100 percent tell you that if it was six months [long], I would not have gone. Because, I could barely make it. I could barely wrap my head around one month before I went.... I told you I was so wrapped tightly in my "bubble" and I have been my entire life that I ... was not mentally prepared to take it to the extreme that I thought one month was. Especially living with a host family. And I think for all of us that was something very, very different because just thinking about all of the people in the group we all were in a "bubble" I think, whether they like to admit it or not. 
Tess, Tim, and Kynzee were the exceptions to this pattern; they all maintained up to a year would have been ideal. The three of them maintained the "pre-trip version" of themselves would have loved the opportunity to study for up to a year. However, they were the three participants who had the most difficult time collecting sufficient money for the one-month program and stated they would not have been able to go due to financial limitations if the program were any longer.

Consequently, I feel Dr. Green carried out the study-abroad program for as long as he possibly could without limiting participants to wealthy participants. Yet, even the upper-middle class participants stated that they could barely "wrap their heads around" a month away from the United States. For all eight of the participants in this program anything longer than one month would have prevented them from going. Therefore, although not ideal for critical social transformations, this study was as long as it could be and still encourage the students to participate.

Different host family "fits" produced different results. One negative outcome I observed was the tendency for the host families to become an essentialized version of Spaniards in the students' discourse (i.e. the host family represented all Spaniards). Therefore, if the host family relationship was positive (Tess, Becky, Kynzee, and Kristene), the participants' view of the Spanish had a tendency toward overlyromanticizing their experience. In contrast, if the host-family experience was negative (Kylie, Anne, and Jennifer for the first two weeks) the host family symbolized a negative version of the essentialized "other." Additionally, I commented in my field notes on multiple occasions that the participants (minus Tim, Kynzee, Tess, and Becky) tended to 
talk about their family as "paid help." One of the negative outcomes of this arrangement is that the students did not have to venture out into society to provide necessities for themselves, which limited their opportunities for linguistic and/or cultural "otherness" (example, Jennifer and water) (Foster, 1997; Morgan, 2010; Pritchard, Morgan, \& Ateljevic, 2011; Ross, 2010). Several of the participants mentioned the possibility of dorms as an alternative, but I believe that a dorm situation would produce even fewer interactions with the host culture because food and housing would be provided and the students would be able to isolate themselves even easier. During the focus-group interview, Jennifer suggested that a cultural exchange might be a solution to reduce costs and encourage greater interaction between the participants and the host culture. She maintained that if the students knew they were going to spend a month or more in each other's houses, it would raise the expectations of interaction and lower the idea of "I'm paying for this" (Anne).

\section{Race, class, and male socio-cultural variables were impossible to analyze in}

isolation. A primary purpose of this study was to analyze what role socio-cultural variables (race, class, gender, language, sexual identity, and cultural heritage) played in the transformative learning of study-abroad students. However, only two of the participants self-identified as Latinas. Furthermore, they were also the participants with the least class privilege out of the study-abroad group. Consequently, it was impossible to isolate class and race variables for those participants during data analysis. Additionally, there was only one male participant, which made it impossible to decipher whether the findings were simply Tim's personality or products of male privilege. 
Therefore, I had to analyze transformative outcomes for both the Latina participants and the male participant comparing their findings to academic literature reviewed for this study. Nevertheless, the process of comparing research findings of small groups to the literature reviewed is at the heart of grounded theory research (Charmaz, 2003; Glaser, 2012; Mills, Bonner, \& Francis, 2006). Furthermore, the small sample size of the study $(N=8)$ made generalizability impossible, and the small size of the male $(n=1)$, Latina $(n$ $=2)$, poverty $(n=1)$, and White $(n=6)$ subgroups may even render the transferability difficult to assess for these findings (Clark \& Cresswell, 2010; Vogt, Gardner, \& Haeffelle, 2012).

As previously mentioned in the Methods chapter, this research did not produce cause-and-effect conclusions that can be replicated by other social scientists. However, the purpose of this study was rich description of eight students in a study-abroad context, not generalizability. Qualitative research typically is best in the initial phases of understanding a phenomenon (Marshall \& Rossman, 2011). Although TLT has been around for more than 50 years, few studies exist that empirically explore travel for transformation (Morgan, 2010). Even fewer studies exist that look at travel for transformation through a socio-cultural lens (Pritchard, Morgan, \& Ateljevic, 2011). Therefore, this study represents the first study that I could find in the literature that specifically examined culture through the lenses of both travel for transformation and CRT to seek understanding how critical social transformation happens. Therefore, as more studies are carried out looking at the socio-cultural variables of the students, 
findings may be gathered from multiple studies like this one to conduct a meta-analysis that can be transferred to other populations of study-abroad participants.

\section{Different amounts and different types of data from different participants.}

There was variety in the type of and a disparity in the amount of data collected from each data source during the study-abroad program. For example, Becky and Tess only came to Ana's class, so I had less information from them during class than I did from the other participants. Likewise, Anne and Kylie talked a lot less during class than the other participants, while Kynzee, Kristene, and Jennifer were more talkative with the LU professors. Kristene asked to be excluded from the Flickr data collection method and asked that I not look at pictures of her that the other participants posted. However, even though there existed a large disparity of data collected within data collection methods, there was almost an even distribution of total data gathered from each participant.

At first, I thought this was my desire to get information from all of the participants, not solely the talkative ones. However, the more I grappled with this, the more I realized that the students wanted their narratives shared. For example, Kylie informed me she was naturally shy in groups, but was very affable when there were just a couple of people present. Therefore, she talked more in interviews (she even offered to do a one-on-one semi-informal interview following the focus-group interview to make up for her silence during the focus group interview). Additionally, Kylie took more pictures on Flickr than any other participant and offered me her blog to make up for the disparity of in-class comments. Likewise, when Kristene withdrew the Flickr data collection method, she offered her personal journal. Her journal gave me insights to what she was 
thinking far more than the Flickr pictures ever would have. Additionally, even though Tess and Becky only took one LU class, they were the most likely to tell me stories of their adventures before and after class.

An overarching theme I observed was the more voluntary their data was, the more they offered it. This is one of the reasons I wished I would have decided to follow their Facebook and Instagram accounts rather than the group Flickr account in retrospect. Several of the students offered, but I did not feel it fit within the confines of the research proposal at the time of the research. As I collected the data for each participant, I noticed that they all averaged about 50 pages of total data (with the exception of Kristene, who gave me a copy of her 89-page, handwritten journal). Because the purpose of this study was descriptive in nature, rather than experimental, I do not feel that the disparity in types of data harmed the outcome of descriptive analysis of what led them towards transformative learning. Furthermore, promoting the narratives of the participants and co-constructing their narratives (in the way they volunteered it) aligns with the constructivist grounded theory data collection and analysis methodology used in this research design (Charmaz, 2003; Pritchard, Morgan, \& Ateljevic, 2011).

Dual-language research methodology. I decided to interview the students in English because I felt it would reduce their cognitive load and provide more nuance in their descriptions of how their experiences were influencing their transformative learning. Even Tess, who was a heritage Spanish speaker, did better explaining her views in English because she had never been taught academic Spanish until she went to college. Very little translation from Spanish to English was necessary because the students spoke 
almost exclusively in English when they were not in class. Knowing that all translations are biased, I had Dr. Green look over any information related to language that emerged in this dissertation. As a certified Oral Proficiency Interview Tester by the American Council on the Teaching of Foreign Languages and professor of Spanish Literature, he supported my observations and translations. Furthermore, I hold a degree in Spanish translation.

The only caveat referring to language limitations Dr. Green offered was he felt that several of the students used me as an "escape" to speak English when they did not feel like speaking Spanish. He also observed that this group spoke in English more than any other group he had ever taken to Spain and felt like their opportunities of speaking with me in English may have negatively impacted their desires to speak Spanish outside of classes (which was a part of their grade for the study-abroad semester). We discussed how this ease of speaking English may have not influenced the students to feel "trapped" or linguistically "othered," which Foster (1997) maintains is a precursor to transformative learning in travel situations.

Friendship with Dr. Green provided institutional access, but slowed gaining trust of the participants and may have limited full disclosure from participants. As stated in the Methods chapter, my friendship with Dr. Green provided instantaneous access to the LU facilities, staff, tour guides, and professors. Levante University provided free attendance for me (and sometimes my family) to all of the cultural excursions. As far as any research goes, I do not think there could be a better "gatekeeper" to institutional access (Marshall \& Rossman, 2011). However, I also 
believe that our friendship delayed access to the participants and may have prevented full disclosure about Dr. Green or the study-abroad program. Several times the participants said things like, "No offense to your friend, Dr. Green" when they were voicing complaints about him or the study-abroad program. Nevertheless, I felt that I did my best to gain the trust of the participants and took them voicing complaints about him or the program to me as an indication that I had gained their trust.

\section{Trustworthiness}

Trustworthiness is qualitative research's conceptual companion to validity in quantitative research (Vogt, Gardner, \& Haeffelle, 2012). Trustworthiness is demonstrated through triangulation, dependability, and confirmability (Marshall \& Rossman, 2011).

Triangulation. As mentioned in the Methods chapter, understanding that I have subjectivities and biases as a researcher, I put into place protocols for managing biases such as triangulation of multiple data points, cross-checking data points, searching for cross-participant themes that emerged, and peer review. Furthermore, I selected a variety of data collection methods from multiple sources to ensure the collection of rich data and to invite the participants to volunteer as much information regarding their experiences as possible. In addition, through consistent and repeated member checks both during and after the study-abroad program, I encouraged the participants themselves to consistently review the data as it was collected and to discuss the meanings of the findings, thereby encouraging them to be co-participative storiers with me in co-constructing the themes that were generated (Pritchard, Morgan, \& Ateljevic, 2011). 
Dependability. I demonstrated dependability of the findings by being explicit with the participants and the reader my role as the researcher, by relating the findings to the literature review, and by seeking the assistance of critical friends throughout the research process. My understanding of CRT, TLT, and transformation for travel literature increased the likelihood of a complex and nuanced description of the patterns and themes that emerged through this study. Furthermore, I asked Dr. Green (who attended all of the cultural excursions and some of the class sessions) to be a critical cross-checker and peer-reviewer of the initial generation of themes produced while data were collected and interviews were used to check emerging themes and patterns. To ensure privacy of the participants, however, we spoke in vague terms so that I did not betray the trust that the participants had in me that I would guard their confidentiality. Furthermore, I obtained the review of two experienced qualitative researchers during data analysis, writing the findings, and preparing and writing this chapter.

Confirmability. Finally, I exhibited confirmability of the findings and conclusions by showing how the interpretations I made directly emerged from the coconstruction of themes based on the systematic collection, analysis, and findings of data (Marshall \& Rossman, 2011). Furthermore, in order to produce confirmable results, I showed the logical manner in which the methodology was created and rooted in the research questions and the purposes of this research project. Moreover, as I wrote the Findings and Discussion chapters, I consistently tied the participants' words and actions to relevant academic literature and empirical research regarding TLT, CRT, and travel for transformation. 


\section{Recommendations for Future Study}

During this section I will examine how the findings and conclusions of this study correspond with, contradict, and/or deepen findings from other relevant academic literature in order to provide recommendations for future study.

“Willingness.” Throughout Mezirow’s writings (1996, 1998, 200, 2012), he maintains "willingness" is a key component in bringing about a disorienting dilemma, which, in his theory, is a pre-requisite to transformative learning. However, reviewing Tim's, Kynzee's, and Becky's data led me to question Mezirow's repeated use of the term "willingness" in his writings. Mezirow (2000) indicated that willingness to listen and speak was a key component in TLT (see also Mezirow, 1996).

Tim was one of the most "willing" participants in the study-abroad program, yet he experienced no stated or observed transformation. His "hell, yeah" attitude was almost palpable and contagious. He was "willing" to discuss, speak, and participate in the classes. To be clear, in the literature reviewed, Mezirow never indicates that "willingness" compels a person to transformation. However, Tim's rabbit-devouring nature made him a prime candidate for thinking about this term. Furthermore, Tim was extremely open to new ideas and both listened and, especially, voiced his own opinions. Remaining silent is one of the conditions that Mezirow (1998) indicates impedes reflective discourse and transformation.

Kynzee’s and Becky’s “willingness” felt different from Tim’s, however. Whereas Tim was a devourer, a taker, of culture, Becky and Kynzee were receivers of it. They valued the host culture, participated in their families' activities, and adapted Spanish 
customs through comportment and dress.

The closest I could find among the TLT theorists to articulate this idea of receiving, rather than taking, was Brookfield (2012), who maintained that participants must listen to a wide range of views and encourage dissention, but provisionally suspend judgment of the issue until all beliefs are stated, thereby exhibiting open-mindedness and a willingness to hear multiple perspectives. The caution to suspend judgment, to encourage, to listen, to hear are all receptive in nature, and are perfect descriptors of Kynzee and Becky. Additionally, O'Sullivan's (2012) critique of Mezirow's overemphasis of reflective discourse in transformation can apply to the receptive nature of Becky and Kynzee. He stated, "There is no question that challenging discussion can stimulate change $\ldots$ however, there are other routes to transformative learning...We also understand that crucial learning often takes place nonverbally, in the inarticulate dimensions of our bodies" (p. 172).

I came to identify with the term "permeability" that Mezirow $(1998,2000)$ uses interchangeably with "willingness" because of the accepted receptive connotation of the word. In addition, permeability more closely aligns with what separated Kynzee and Becky from Tim. Furthermore, as I reviewed the data, I realized that the finding that cultural inclusion, rather than "otherness," had a more profound effect on the transformative outcomes for several of the participants also reflected a change in permeability. For example, both Anne and Jennifer were closed off to new ideas until they experienced cultural inclusion. This inclusion changed their stated dispositions toward receiving culture, toward permeability. 
In sum, the findings regarding "willingness" led me to believe Mezirow's "willingness" is unfocused, in need of refinement, further definition, and reframing. Moreover, this aspect of his theory is in need of further empirical analysis through future studies. With the insight that permeability (or receiving) is a condition that opens a person to transformative learning, is this a personal characteristic or something that can be fostered? If it can be fostered, how can opportunities be designed that encourage permeable, rational worldviews that TLT scholars discuss as a prerequisite to transformative learning (Ettling, 2006; Mezirow, 2000, 2004, 2012; O'Sullivan, 1999; Taylor \& Cranton, 2012)? How can study-abroad programs design opportunities to serve as a catalyst for transformation in less permeable students? Is cultural inclusion, rather than "otherness," a catalyst for instigating permeability in study-abroad students? Therefore, I recommend future studies be carried out to clarify, refine, and reframe what is meant by "willingness" in TLT, what role it plays in transformation, and how it can be fostered in participants.

Social constructivism, rather than constructivism. Jennifer's culturally oriented transformation made me question what role social constructivist underpinnings may play in critical social TLT. Jennifer told me that all of her complaints and concerns about the study-abroad program disappeared during the festival weekend with her host "sister," Magdalena. In the literature review, I wrote that constructivism (one of the paradigmatic underpinnings of TLT) looks at how an individual's mind perceives and responds to social stimuli, while social constructivism sees the lower level biologicalmental functions being formed and developed by sociocultural influences (Matusov \& 
Hayes, 2000). It seems that Jennifer's experiences with Magdalena allowed the culture of Spain to permeate Jennifer's worldview. This is more closely related to Freire's (2000) social constructivist concept of “conscientização," wherein he describes society framing transformation, rather than Mezirow's (2000) view of transformation commencing from an inward personal disorienting dilemma. Social constructivists, like Freire, see learning as an active social and cultural process whereby learning comes from a shared sense of reality (Kezar, Carducci, \& Contreras-McGavin, 2006).

It would be impossible to separate Jennifer's sense of social inclusion in a vacuum void of Magdalena (similarly, it would be impossible to separate Anne's sense of social inclusion without Tess and Paco). Therefore, her transformation toward social inclusion may not be classical transformative learning in the original constructivist sense, but rather a socio-cultural shift in epistemology due to newfound, shared values that permeated her co-consciousness. Nevertheless, the transformation was easily observable, even if the root cause was different than Mezirow's constructivist paradigmatic underpinning. Jennifer's culturally-oriented transformation made me question what role social constructivist underpinnings may play in critical social TLT and is a recommendation for future studies looking at how critical social TLT differs in epistemological underpinnings from Mezirow's constructivist epistemological underpinning.

Could study abroad, be study across town? In the findings regarding Tess' experiences, I wrote: 
Ironically, the culture the PSU students sought to discover through the studyabroad program existed twenty minutes away from their suburbs and sat a few feet away from them in their PSU Spanish classes long before the study-abroad program ever started.

This observation led me to question if students needed to travel across the world, if sociocultural transformation is the desired outcome of study-abroad. It seems logical that a visit to parts of town that students feel are "other" may have an equal—or more powerful—outcome in understanding and acting to disrupt social disparities (Slattery, 2013). Visits to parts of town that participants' view as "other" may produce equally "novel" destinations that pull a participant away from known experiences (Ross, 2010). To clarify, I must distinguish between "other" and "other-ed" before I continue. "Other" signifies novel or cognitively removed for the participant (a technophile in a natural setting, for example). However, "other-ed" indicates already oppressed peoples due to social, educational, political, or economic institutions that promote the normativity of White, upper-middle class values. Furthermore, the intercultural dialogues, reflections, and intimate intercultural experiences required in travel for transformation could more naturally and logically be transferred "home." Consequently, reflections with previously "other" (to the participant) communities could lead to critical reflection and breaking down barriers to equity within the participants' own culture.

Moreover, this opportunity for intercultural dialogue may spur some radical cultural inclusion, like what occurred for Tess, Kynzee, Jennifer, Kristene, and Becky. This sense of inclusion would be a quintessential representation of subjective reframing 
(which was missing from many of the students' study-abroad experience) because the participants would be acting to improve society for individuals they previously perceived as "other" within their own community. Also, this kind of dialogue would remove the "pedagogy for the privileged" necessity because transportation to an "other" (to the participant) place within the same city would be exponentially more affordable and open access to all students.

Although this form of travel transports an individual to an "other" (for the participant) place within her or his community, my grapple with this suggestion for future study is the potential to reify already existing modes "taking" from already taken-from peoples. With study abroad, if the "pedagogy of the privileged" converts into voyeurism or appropriation (as it did with several of the participants in this dissertation research), the participants at least have limited realistic chances for interacting with the host culture in the future. In contrast, when the host culture is one's community of origin, the potential harmful effects of essentialism, stereotyping, or tokenizing exponentially increase (but so do the potential gains). Therefore, the same cautions that exist for travel for transformation would need to be explicitly followed in a travel to an "other" (for the participant) place within an individual's own community: the travel experience must respect the values and knowledge of the host culture, acknowledge the presence of differences in privilege, and utilize environmentally sustainable practices (Ross, 2010). In addition, the duration, purpose of travel, and degree of immersion still plays a significant role in perspective transformation (Brown, 2009). 
Social justice class before travel. Cordero and Rodriguez (2007) maintain that a study-abroad program promotes greater critical social TLT outcomes when they teach a class on social justice before the participants travel. In fact, they see the study-abroad program as an extension of their class on social justice. Furthermore, a class on societal power structures can promote the awareness of the harmful effects of stereotyping, essentializing, and microagressions that are common when participants attempt to immerse themselves in a culture that feels "other" or novel to them (Gambrell \& Bright, in press).

Even though the stated purpose of the study-abroad program was to learn Spanish, Dr. Green manifested a strong personal value of critical social transformative learning in his students. I believe several of the participants (Tim, Kristene, and Jennifer) shared political values that could motivate them to be powerful allies for racial, gender, sexual, and class justice. However, at the time of the study-abroad program, these students were not yet capable of seeing how their own privileged worldviews reified the injustices that contradicted their political ideology (Brookfield, 2012; Leonardo, 2012; McIntosh, 1990; Mezirow, 2012). Nevertheless, a program directed with a specific critical social outcome, would be worth future study.

Reflection before, during, and after the study-abroad program. If a class specifically addressing social justice were not a reality for a program (for example, Dr. Green's Spanish study-abroad program), reflection during and after the study-abroad program could promote critical reflection (see also Ross, 2010). Mezirow (1997, 1998, 2000) maintains that critical reflection is a key component to transformation. The 
findings in this research concur. For example, Tess explained in her member-check interview that the capacity to reflect through dialogue with me during and after the studyabroad program increased her understanding of the importance of this trip for understanding (and hopefully eventually overcoming) a lifetime of intersecting marginalizations. Also, Anne's time in her room provided her with the space to reflect on her lifetime of abuse and she was able to emancipate herself from a lifetime of guilt. Additionally, Kristene wrote several times that she valued the time to read and to write in her journal that the study-abroad experience granted her.

These findings leave me pondering what I could have done better to create opportunities for critical reflection. Ross (2010) maintains that post-travel activities to help the sojourner to continue to reflect upon and extract meaning from the travel foster transformation. I posit that a longitudinal study one year, five years, and ten years after the study-abroad program would offer opportunities for the participant to critically reflect upon the transformative learning that took place during travel. Furthermore, a longitudinal design would extend the timeline of the research, providing a better picture of what transformations were related to travel for transformation, but that occurred much later than the event itself (Tisdell, 2012). Additionally, a self-reflective tool like a journal or a blog would likely increase opportunities for critical reflection during (and even after) the study-abroad program.

Cultural and linguistic inclusion is under-theorized. Research regarding cultural and linguistic inclusion, rather than "otherness," was absent from the literature reviewed and available at the time of this dissertation relating to study abroad. I found 
academic literature related to classroom inclusion for fostering transformation with students from varying racial and class constructs (Ettling, 2006; hooks, 1994; JohnsonBailey \& Alfred, 2006). However, in specific relation to travel for transformation, few sources exist that address cultural inclusion. For example, Goulah (2007) found that students who learned another language while immersed in another culture developed critical socio-cultural attitudes about their home culture. Additionally, Foster (1997) maintained that students who learned another language had to develop the skill to think like another culture. Also, Ross (2010) theorized that being willing to be seen like a representative of the host culture increased transformative learning. Yet, the participants of this research manifest greater socio-cultural transformation through linguistic and cultural inclusion, rather than "otherness." Therefore, I posit that research regarding study abroad and cultural or linguistic inclusion, rather than "otherness," is undertheorized and in need of further study. As such, I recommend conducting empirical studies focused on the critical social impacts on transformation from linguistic and cultural inclusion in study-abroad programs.

\section{Further studies to analyze how social constructs of the participants impact}

transformation. As stated in the literature review, many scholars argue that Mezirow's model of TLT is overly individualistic (Brookfield, 2012), gendered (English \& Irving, 2012), raced (Cranton \& Taylor, 2012; Johnson-Bailey \& Alfred, 2006), self-centered rather than earth-centered (O'Sullivan, 2012), and North American (or Western value)centered (Ntseane, 2012). In addition, E. W. Taylor (2007) states that studies have shown that social context (race, class, gender, and culture) may be the most important variable 
on transformative learning outcomes. This study's findings about race and class support his position. However, it represents the first study that specifically examined culture through the lenses of both travel for transformation and CRT to seek understanding how critical social transformation happens. Therefore, more empirical analysis is necessary to engage theoretical frameworks beyond Mezirow's dominant perspective to motivate participants toward social action (Taylor \& Snyder, 2012). More importantly, I recommend that future studies be conducted to continue to focus on how race, gender, sexuality, cultural identity, language, and other socio-cultural variables impact critical social transformation.

\section{Significance of the Study}

The purpose of this research was to identify what elements of study-abroad programs have the potential for critical social transformative learning. While many colleges and universities implement study-abroad programs with the underlying assumption that they are transformative, little empirical research has been done to examine the elements of travel that contribute to transformation (Ross, 2010). Even fewer studies have been carried out to explore the socio-cultural variables of race, gender, sexuality, class, and (dis)ability and how they impact travel for transformation (Pritchard, Morgan, \& Ateljevic, 2011). This dissertation was an initial attempt to generate theory pertaining to travel for transformation through a critical social lens.

Most university departments do not have transformative learning as the purpose of study-abroad programs (for example, this study-abroad program's purpose was to improve Spanish language proficiency). However, TLT's axiology of humanism values 
all learning's potential transformative outcomes in which the participants can become more reasonable, autonomous, self-actualized, and have a responsibility to make their own life better while also improving the conditions of those around them (Cranton \& Taylor, 2012). Additionally, TLT's underpinning of a critical social ontology urges individual epistemology to be tied to political action to improve society (Brookfield, 2012). Therefore, TLT lies at the intersection of personal and social, which hopefully leads participants to a greater sense of social accountability and action (Taylor \& Snyder, 2012). According to Mezirow (2012), these critical social outcomes are a powerful part of all adult learning, regardless of the content matter.

The results of this study and others like it could assist universities in deciding whether study-abroad programs are a "pedagogy for the privileged" for the few who can afford to undertake such journeys and/or a vehicle for critical socio-cultural transformation (Curry-Steven, 2007). Consequently, university decision-makers can understand how the social context of the participants and the host culture influence travel for transformation to avoid recreating marginalization and oppression through studyabroad programs.

\section{Summary}

Transformative learning theory describes the process of reframing discriminative, untenable worldviews with a more inclusive, permeable, and reflective epistemology. Although transformative learning theory (TLT) has been around for more than 50 years, few studies exist that empirically explore transformation through a sociocultural lens. Recent (2006-2012) TLT researchers are looking beyond Mezirow for 
other theoretical orientations of transformative learning (Taylor \& Snyder, 2012). Although studies have shown that social context may be the most important variable on transformative learning outcomes (Taylor, 2007), few studies explored the impact the participants' background, culture, and/or political positionality related to transformative learning" (Taylor \& Snyder, 2012). Therefore, it was imperative to investigate what role participants' backgrounds have in transformative learning, especially what role race, language, class, and culture played in cognitive dissonance.

A pre-existing theoretical framework for analyzing how race and culture affect transformative learning is CRT. Critical Race Theory is a form of oppositional scholarship used to "uncover racial inequity and legal injustice" which "inform strategies of resistance" (Taylor, 2006, p. 72, see also, Bell, 2009). Repeated themes in CRT scholarship include: deconstruction of the myths of colorblindness within educational institutions; promotion of voices and narratives of people of color; interest convergence; exposure of racism as normal — meaning both universal and embedded within statutes and policies; and exposure of White, middle-class values as normative (Delgado, 2009b; Ladson-Billings, 1998; Litowitz, 2009; Smith \& Stovall, 2008; Taylor, 2006). The myths of colorblindness, post-racial society, and meritocracy mask the dominance those who are socially constructed as White have over people of color in United States society. The omni-presence of White supremacy makes it difficult for privileged classes to see political, legal, financial, and social benefits of being White (Gillborn, 2009). Because it is a well-established theory regarding race and other forms of systemic oppression, CRT was well suited as a framework for analyzing and giving vocabulary to how race (and 
other socio-cultural variables) informed the way students transformed (or did not transform) into more tolerant ways of seeing the world and acting in it (Bright \& Gambrell, 2013).

A growing body of research explores the effects of travel, tourism, and studyabroad programs on transformation of cultural worldview (Coghlan \& Gooch, 2011; Cordero \& Rodriguez, 2009; Falk, Ballantine, Packer, \& Brinckerhoff, 2012; Morgan, 2010; Pritchard, Morgan, \& Ateljevic, 2011; Ross, 2010). Because travel situates the learner in the position of "other" and that most travel is done with a purpose for personal growth, travel (especially where one has to learn another language) is especially well suited to transformative learning (Morgan, 2010; Foster, 1997). Previous research indicates that the duration, purpose of travel, and degree of immersion plays a significant role in perspective transformation (Brown, 2009). Duration of time in an "other" culture also has an impact on transformation: in Cordero and Rodriguez' (2009) twelve-day study-abroad program not every student experienced transformation, whereas every student in the Brown's (2009) study where participants spent a year immersed in the host culture experienced irreversible transformative learning. It is also important that the transformative travel educator understands that study-abroad research is a "pedagogy of the privileged" wherein students with access to funding for travel are coming to understand their role as members of dominant society within an oppressive culture (Coghlan \& Gooch, 2011).

Through a CRT framework, this study analyzed how socio-cultural variables (race, class, gender, sexuality, and language) impacted the transformative learning of 
study-abroad students. Therefore, the purpose of this study was to describe to what extent eight students from a medium-sized, rural Midwestern university experienced the elements of transformative learning during a month-long study-abroad experience in Spain. In addition, this study explored what the experience of traveling to an "other" place and experiencing "otherness" had on transformative learning. Finally, this research examined how each participant's racial, economic, gender, and cultural identity impacted her or his worldview and unpacked how these social variables affected the transformation process.

These research questions were answered through a qualitative research methodology incorporating biological narratives, photo/video analysis, extensive ethnographic observations, surveys, and informal, semi-formal, and focus group interviews. The data were analyzed using Charmaz's (2003) constructivist grounded theory. The participants under this construct became co-participative storytellers with the researcher in the generation of codes, patterns, and overarching themes. Following the study, data were scrutinized searching for salient themes, patterns, or other relevant information. Furthermore, the patterns and themes were compared and contrasted with studies cited in current academic literature regarding critical social TLT, CRT, and travel for transformation. Data analysis was an ongoing and cyclic process that happened from the first day of the study-abroad program through the writing of this chapter.

Race and class privilege had an observable impact on the study-abroad participants' worldviews and transformative learning. Despite feeling culturally "othered" by members of Spanish society, the White, upper-middle class participants 
made no subjective leap to how racially, linguistically, and economically marginalized individuals are culturally "othered" in the United States and how their own epistemology, rooted in unearned privilege, might reinforce that marginalization. In contrast to cultural "otherness," perceived linguistic "otherness" created opportunities for these same students to subjectively reframe their discriminatory attitudes about speakers of other languages. Moreover, cultural inclusion had more impact than "otherness" for transforming cultural frames of reference.

I additionally observed that the White, upper-middle class participants experienced epochal transformations that were personal transformations rather than critical social transformations. All of these experiences were personally powerful and aligned with Mezirow's $(2000,2012)$ version of TLT in that the students became more autonomous. However, while the participants' subjective transformation was deeply engaging and important on a personal level, it did not appear to motivate them toward more justified beliefs or action toward others. Nonetheless, although many of the students had not stated a desire to enact social change at that time, I believe that sociocultural reframing may be yet a possible outcome of this study (Tisdell, 2012).

The findings of this study reinforce Johnson-Bailey and Alfred's (2006) theory that marginalization leads to critical reflection and subjective reframing. However, marginalization alone did not result in critical social outcomes. Rather, socio-cultural variables of racial identity and class (which is at the heart of Johnson-Bailey and Alfred's article) and the lifelong experiencing with "otherness" had a greater impact on the critical social reflection of both Latina students. Thus, a surprising outcome of this study for me 
as a researcher was being forced to confront the Black vs. White binary that is often the dominant narrative in CRT scholarship (Ladson-Billings, 2009). More than ever before, I came to understand that race is a social (identity), not biological (skin color), construct. Although many prefer to categorize identities into single social mechanisms, intersections of marginalizations (or intersections between privilege and marginalization) provide powerful rich new categories that need to be studied and understood (Crenshaw, 2009).

The findings of this study led me to question if students needed to travel across the world, if socio-cultural transformation is the desired outcome of study-abroad. It seems logical that a visit to parts of town that students feel are "other" may have an equal—or more powerful—outcome in understanding and acting to disrupt social disparities. In addition, requiring students to take a class about equity and social justice may lead to broader social justice objectives when combined with a study-abroad experience (Cordero \& Rodriguez, 2009)

In conclusion, I recommend conducting further empirical studies focused on the critical social impacts on transformation from linguistic and cultural inclusion in studyabroad programs.

More empirical analysis is necessary to engage theoretical frameworks beyond Mezirow's dominant perspective to help create allies for social justice (Taylor \& Snyder, 2012). Most importantly, I recommend that future studies be conducted to continue to focus on how race, gender, sexuality, cultural identity, language, and other socio-cultural variables impact critical social transformation. 


\section{References}

Adams, M., Blumenfield, W. J., Castañeda, C. R., Hackman, H. W., Peters, M. L., \& Zúñiga, X. (2010). Readings for diversity and social justice. New York, NY: Routledge.

Alhadeff-Jones, M. (2012). Transformative learning and the challenges of complexity. In E. W. Taylor \& P. Cranton (Eds.), The handbook of transformative learning: Theory, research, and practice (pp. 178-194). San Francisco, CA: Jossey-Bass. Anzaldúa, G. (1990). Haciendo caras, una entrada. In G. Anzaldúa (Ed.), Making face, making soul: Creative and critical perspectives by feminists of color (xv-xxviii). San Francisco, CA: Aunt Lute Books.

Baumgartner, L. M. (2001). An update on transformational learning. New Directions for Adult and Continuing Education, 89(2), 15-24.

Baumgartner, L. M. (2012). Mezirow's theory of transformative learning from 1975 to present. In E. W. Taylor \& P. Cranton (Eds.), The handbook of transformative learning: Theory, research, and practice (pp. 99-115). San Francisco, CA: Jossey-Bass.

Bell, D. A. (2009). Who's afraid of critical race theory? In E. Taylor, D. Gillborn, \& G. Ladson-Billings (Eds.), Foundations of critical race theory in education (pp. 3750). New York, NY: Routledge.

Bell, Jr., D. A. (2009). Brown v. Board of Education and the interest convergence dilemma. In E. Taylor, D. Gillborn, \& G. Ladson-Billings (Eds.), Foundations of critical race theory in education (pp. 73-84). New York, NY: Routledge. 
Bennetts, C. (2003). The impact of transformational learning on individuals, families and communities. International Journal of Lifelong Education, 22(5), 457-480.

Blumenfeld, W. (2006). Christian privilege and the promotion of "secular" and not-so "secular" mainline Christianity in public schooling and in the larger society. Equity \& Excellence in Education, 39(3), 195-210.

Bolman, L. G., \& Deal, T. E. (2008). Reframing organizations: Artistry, choice, and leadership. San Francisco, CA: Jossey-Bass.

Boncana, M. (2010). Participation, student achievement, and parental involvement in a Utah elementary school: Multilevel growth curve and critical interpretive analyses (Unpublished doctoral dissertation). University of Utah, Salt Lake City, UT.

Brah, A., \& Phoenix, A. (2009). Ain't I a woman? Revisiting intersectionality. In E. Taylor, D.

Gillborn, \& G. Ladson-Billings (Eds.), Foundations of critical race theory in education (pp. 247-257). New York, NY: Routledge.

Bright, A., \& Gambrell, J. A. (2013, November). Erasing myths of justice and meritocracy:

Embracing critical race theory in teacher education. Workshop presented at the annual meeting of the National Association for Multicultural Education, Oakland, CA.

Brookfield, S. D. (1998). Critically reflective practice. Journal of Continuing Education in the Health Professions, 18(4), 197-205. 
Brookfield, S. D. (2002). Using the lenses of critically reflective teaching in the community college classroom. New Directions for Community Colleges, 118, 3138.

Brookfield, S. D. (2012). Critical theory and transformative learning. In E. W. Taylor \& P. Cranton (Eds.), The handbook of transformative learning: Theory, research, and practice (pp. 131-146). San Francisco, CA: Jossey-Bass.

Brown, K. M. (2006). Leadership for social justice and equity: Evaluating a transformative framework and andragogy. Educational Administration Quarterly, 42(5), 700-745.

Brown, L. (2009). The transformative power of the international sojourn: An ethnographic study of the international student experience. Annals of Tourism Research, 36(3), 502-521.

Case, R. (1992). Neo-Piagetian theories of child development. In R. J. Sternberg \& C. A. Berg (Eds.), Intellectual development (pp. 161-196). Cambridge, England: Cambridge University Press.

Charmaz, K. (2003). Grounded theory: Objectivist and constructivist methods. In N. K. Denzin \&Y. S. Lincoln (Eds.), Strategies of qualitative inquiry (pp. 249-291). Thousand Oaks, CA: Sage.

Clark, V. L. P., \& Creswell, J. W. (2010). Understanding research: A consumer's guide. Upper Saddle River, NJ: Pearson Educational, Inc. 
Clover, D. E. (2006). Out of the dark room participatory photography as a critical, imaginative, and public aesthetic practice of transformative education. Journal of Transformative Education, 4(3), 275-290.

Cochran-Smith, M. (2001). Constructing outcomes in teacher education: Policy, practice, and pitfalls. Education Policy Analysis Archives, 9(11), 1-56.

Cochran-Smith, M., Shakman, K., Jong, C., Terrell, D., Barnatt, J., \& McQuillan, P. (2009). Good and just teaching: The case for social justice in teacher education. American Journal of Education, 115(3), 347-377.

Coghlan, A., \& Gooch, M. (2011). Applying a transformative learning framework to volunteer tourism. Journal of Sustainable Tourism, 19(6), 713-728.

Cole, M. (1985). The zone of proximal development: Where culture and cognition create each other. In J. V. Wertsch (Ed.), Culture, communication, and cognition: Vygotskian perspectives (pp. 146-161). Cambridge, England: Cambridge University Press.

Cordero, A., \& Rodriguez, L. N. (2009). Fostering cross-cultural learning and advocacy for social justice through an immersion experience in Puerto Rico. Journal of Teaching in Social Work, 29(2), 134-152.

Courtenay, B. C., Merriam, S. B., \& Reeves, P. M. (1998). The centrality of meaningmaking in transformational learning: How HIV-positive adults make sense of their lives. Adult Education Quarterly, 48(2), 65-84.

Cranton, P., \& Taylor, E. W. (2012). Transformative learning theory: Seeking a more unified theory. In E. W. Taylor \& P. Cranton (Eds.), The handbook of 
transformative learning: Theory, research, and practice (pp. 3-20). San Francisco, CA: Jossey-Bass.

Crenshaw, K. (2009). Intersectionality, identity politics, and violence against women of color. In E. Taylor, D. Gillborn, \& G. Ladson-Billings (Eds.), Foundations of critical race theory in education (pp. 213-246). New York, NY: Routledge.

Crozier, G. (2003). Researching black parents: Making sense of the role of research and the researcher. Qualitative Research, 31(1), 79-94.

Curry-Stevens, A. (2007). New forms of transformative education: Pedagogy for the privileged. Journal of Transformative Education, 5(1), 33-58.

Darling-Hammond, L. (2007). Race, inequality and educational accountability: The irony of "No Child Left Behind." Race Ethnicity and Education, 10(3), 245-260.

Delgado, R. (2009a). Affirmative action as a majoritarian device: Or, do you really want to be a role model? In E. Taylor, D. Gillborn, \& G. Ladson-Billings (Eds.), Foundations of critical race theory in education (pp. 109-116). New York, NY: Routledge.

Delgado, R. (2009b). On telling stories in school: A reply to Farber and Sherry. In E. Taylor, D. Gillborn, \& G. Ladson-Billings (Eds.), Foundations of critical race theory in education (pp. 340-348). New York, NY: Routledge.

Delgado, R., \& Stefancic, J. (2001). Critical race theory: An introduction. New York, NY: New York University Press. 
Delgado-Bernal, D. (2002). Critical race theory, Latino critical theory, and critical racedgendered epistemologies: Recognizing students of color as holders and creators of knowledge. Qualitative Inquiry, 8(1), 105-126.

Delgado-Gaitan, C. (1993). Researching change and changing the researcher. Harvard Educational Review, 63(4), 389-411.

Dewey, J. (1910). How we think. Boston, MA: D.C. Heath \& Co.

Dirkx, J. M. (2012). Nurturing soul work: A Jungian approach to transformative learning. In E. W. Taylor \& P. Cranton (Eds.), The handbook of transformative learning: Theory, research, and practice (pp. 116-130). San Francisco, CA: Jossey-Bass.

Dudziak, M. L. (2009). Desegregation as a Cold War imperative. In E. Taylor, D. Gillborn, \& G. Ladson-Billings (Eds.), Foundations of critical race theory in education (pp. 85-97). New York, NY: Routledge.

English, L. M., \& Irving, C. J. (2012). Women and transformative learning. In E. W. Taylor \& P. Cranton (Eds.), The handbook of transformative learning: Theory, research, and practice (pp. 245-260). San Francisco, CA: Jossey-Bass.

Ettling, D. (2006). Ethical demands of transformative learning. New Directions for Adult and Continuing Education, 109, 59-67.

Falk, J. H., Ballantyne, R., Packer, J., \& Benckendorff, P. (2012). Travel and learning: A neglected tourism research area. Annals of Tourism Research, 39(2), 908-927. 
Fenwick, T. J. (2000). Expanding conceptions of experiential learning: A review of the five contemporary perspectives on cognition. Adult Education Quarterly, 50(4), 243-272.

Fosnot, C. T., \& Perry, R. S. (2005). Constructivism: A psychological theory of learning. New York, NY: Teachers College, Columbia University.

Foster, E. (1997). Transformative learning in adult second language learning. New Directions for Adult and Continuing Education, 74, 33-40.

Freire, P. (2000). Pedagogy of the oppressed. New York, NY: Continuum International Publishing Group.

Gall, M. D., Gall, J.P., \& Borg, W. R. (2007). Educational research: An introduction. Boston, MA: Pearson Education, Inc.

Gamson, J. (2003). Sexualities, queer theory, and qualitative research. In N. K. Denzin \& Y. S. Lincoln (Eds.), The landscape of qualitative research: Theories and issues (pp. 398-432). Thousand Oaks, CA: Sage Publications.

Gambrell, J. A., \& Bright, A. (in press). Epistemology of radical love: A critical race framework for dismantling the uncomfortable complexities of educator privilege. In J. Diem (Ed.), The Social and Cultural Foundations of Education.

Gillborn, D. (2009). Education policy as an act of white supremacy: Whiteness, critical race theory, and education reform. In E. Taylor, D. Gillborn, \& G. LadsonBillings (Eds.), Foundations of critical race theory in education (pp. 51-72). New York, NY: Routledge. 
Glaser, B. G. (2012). Constructivist grounded theory? The Grounded Theory Review, 11(1), 28-38.

Gold, R. L. (1958). Roles in sociological field observations. Social Forces, 36(3), 217223.

Goulah, J. (2007). Village voices, global visions: Digital video as a transformative foreign language learning tool. Foreign Language Annals, 40(1), 62-78.

Guba, E. G., \& Lincoln, Y. S. (2003). Paradigmatic controversies, contradictions, and emerging confluences. In N. K. Denzin \& Y. S. Lincoln (Eds.), The landscape of qualitative research: Theories and issues (pp. 253-291). Thousand Oaks, CA: Sage Publications.

Harris, K. R., \& Graham, S. (1994). Constructivism: Principles, paradigms, and integration. The Journal of Special Education, 28(3), 233-247.

Harris, C. I. (1993). Whiteness as property. Harvard Law Review, 106(8), 1707-1791. hooks, b. (1994). Teaching to transgress: Education as the practice of freedom. New York, NY:

Routledge.

Jensen, B. (2004). Across the great divide: Crossing classes and clashing cultures. In M. Zweig (Ed.), What's class got to do with it? American society in the twenty-first century (pp. 168-183). Ithaca, NY: Cornell University Press.

Johnson D. W., \& Johnson R. T. (1989). Cooperation and competition. Edina, MN: Interaction Book Company. 
Johnson, R. R. (2003). Autobiography and transformative learning narrative in search of self. Journal of Transformative Education, 1(3), 227-244.

Johnson-Bailey, J. (2012). Positionality and transformative learning: A tale of inclusion and exclusion. In E. Taylor \& P. Cranton (Eds.), The handbook of transformative learning: Theory, research, and practice (pp. 260-273). San Francisco, CA: Jossey-Bass.

Johnson-Bailey, J., \& Alfred, M. (2006). Transformational teaching and the practices of black women adult educators. New Directions for Adult and Continuing Education, 109, 49-58.

Kagan, S. (1994). Cooperative learning. San Clemente, CA: Kagan Publishing.

Kegan, R. (2000). What “form” transforms? A constructive-developmental approach to transformative learning. In J. Mezirow (Ed.), Learning as transformation (pp. 3570). San Francisco, CA: Jossey-Bass.

Kezar, A. J., Carducci, R., \& Contreras-McGavin, M. (2006). Rethinking the "L" word in higher education: The revolution in research on leadership. San Francisco, CA: Jossey-Bass.

Kezar, A. J., \& Eckel, P. (2008). Advancing diversity agendas on campus: Examining transactional and transformational presidential leadership styles. International Journal of Leadership in Education, 11(4), 379-405.

Knowles, M. S. (1970). The modern practice of adult education. New York, NY: Association Press. 
Ladson-Billings, G. J. (1998). Just what is critical race theory and what's it doing in a nice field like education? International Journal of Qualitative Studies in Education, (11)1, $7-24$.

Ladson-Billings, G. J. (2003). Racialized discourses and ethnic epistemologies. In N. K. Denzin \& Y. S. Lincoln (Eds.), The landscape of qualitative research: Theories and issues ( $2^{\text {nd }}$ ed., pp. 398-432). Thousand Oaks, CA: Sage Publications

Ladson-Billings, G. J., \& Donner, J. (2005). The moral activist role of critical race theory scholarship. In N. K. Denzin \& Y. S. Lincoln (Eds.), Sage handbook on qualitative research ( $3^{\text {rd }}$ ed., pp. 279-301). Thousand Oaks, CA: Sage Publications.

Laible, J. C. (2000). A loving epistemology: What I hold critical in my life, faith and profession. International Journal of Qualitative Studies in Education, 13(6), 683692.

Lange, E. A. (2012). Transforming transformative learning through sustainability and the new science. In E. W. Taylor \& P. Cranton (Eds.), The handbook of transformative learning: Theory, research, and practice (pp. 195-211). San Francisco, CA: Jossey-Bass.

Lave, J. (1996). Teaching, as learning, in practice. Mind, Culture, and Activity, 3(3), 149164.

Leithwood, K., \& Jantzi, D. (2005). A review of transformational school leadership research 1996-2005. Leadership and Policy in Schools, 4(3), 177-199. 
Leonardo, Z. (2009). The color of supremacy: Beyond the discourse of "white privilege." In E.

Taylor, D. Gillborn, \& G. Ladson-Billings (Eds.), Foundations of critical race theory in education (pp. 261-276). New York, NY: Routledge.

Litowitz, D. E. (2009). Some critical thoughts on critical race theory. In E. Taylor, D. Gillborn, \& G. Ladson-Billings (Eds.), Foundations of critical race theory in education (pp. 340-348). New York, NY: Routledge.

Liu, A. (2011). Unraveling the myth of meritocracy within the context of U.S. higher education. Higher Education, 62(4), 383-397.

Lopez, G. R. (2003). The (racially neutral) politics of education: A critical race theory perspective. Educational Administration Quarterly, 39(1), 68.

Marina, B. H., \& Holmes, N. D. (2009). Bottom line: Education is the great equalizer. Or is it? About Campus, 14(3), 29-32.

Marshall, C., \& Gerstl-Pepin, C. (2005). Re-framing educational politics for social justice. Boston, MA: Pearson Education, Inc.

Marshall, C., \& Rossman, G. B. (2011). Designing qualitative research (5 ${ }^{\text {th }}$ ed.). Thousand Oaks, CA: Sage Publications.

Matusov, E., \& Hayes R. (2000). Sociocultural critique of Piaget and Vygotsky. New Ideas in Psychology, 18, 215-239.

Mayrowetz, D. (2008). Making sense of distributed leadership: Exploring the multiple usages of the concept in the field. Educational Administration Quarterly, 44(3), 424-435. 
McIntosh, P. (1990). White privilege: Unpacking the invisible knapsack. Independent School, 49(2), 31 .

McNamee, S. J., \& Miller Jr., R. K. (2004). The meritocracy myth. Sociation Today, 2(1). Retrieved from: http://www.ncsociology.org/sociationtoday/v21/merit.htm

Merriam, S. B. (2004). The role of cognitive development in Mezirow's transformational learning theory. Adult Education Quarterly, 55(1), 60-68.

Merriam, S. B., \& Kim, S. (2012). Studying transformative learning theory: What methodology? In E. W. Taylor \& P. Cranton (Eds.), The handbook of transformative learning: Theory, research, and practice (pp. 56-72). San Francisco, CA: Jossey-Bass.

Mezirow, J. (1996). Toward a learning theory of adult literacy. Adult Basic Education, 6(3), 115-126.

Mezirow, J. (1997). Transformative learning: Theory to practice. New Directions for Adult and Continuing Education, 74, 5-12.

Mezirow, J. (1998). On critical reflection. Adult Education Quarterly, 48(3), 185-198.

Mezirow, J. (2000). Learning to think like an adult: Core concepts of transformation theory. In J. Mezirow (Ed.), Learning as transformation (pp. 3-34). San Francisco, CA: Jossey-Bass.

Mezirow, J. (2004). Forum comment on Sharan Merriam's “The role of cognitive development in Mezirow's transformational learning theory." Adult Education Quarterly, 55(1), 69-70. 
Mezirow, J. (2012). Learning to think like an adult: Core concepts of transformation theory. In E. W. Taylor \& P. Cranton (Eds.), The handbook of transformative learning: Theory, research, and practice (pp. 73-96). San Francisco, CA: JosseyBass.

Mills, J., Bonner, A., \& Francis, K. (2006). The development of constructivist grounded theory. International Journal of Qualitative Methods, 5(1), 1-10.

Morgan, A. D. (2010). Journeys into transformation travel to an "other" place as a vehicle for transformative learning. Journal of Transformative Education, 8(4), 246-268.

Ntseane, P. G. (2012). Transformative learning theory: A perspective from Africa. In E. W. Taylor \& P. Cranton (Eds.), The handbook of transformative learning: Theory, research, and practice (pp. 274-288). San Francisco, CA: Jossey-Bass.

O'Sullivan, E. (1999). Transformative learning: Educational vision for the $21^{\text {st }}$ Century. Toronto, Canada: Zed Books.

O’Sullivan, E. (2012). Deep transformation: Forging a planetary worldview. In E. W. Taylor \& P. Cranton (Eds.), The handbook of transformative learning: Theory, research, and practice (pp. 162-177). San Francisco, CA: Jossey-Bass.

Parker, L., \& Lynn, M. (2009). What's race got to do with it? Critical race theory's conflicts with and connections to qualitative research methodology and epistemology. In E. Taylor, D. Gillborn, \& G. Ladson-Billings (Eds.), Foundations of critical race theory in education (pp. 148-162). New York, NY: Routledge. 
Piaget, J., \& Inhelder, B. (2000). The psychology of the child. New York, NY: Basic Books.

Pritchard, A., Morgan, N., \& Ateljevic, I. (2011). Hopeful tourism: A new transformative perspective. Annals of Tourism Research, 38(3), 941-963.

Ravitch, D. (2010). The death and life of the great American school system: How testing and choice are undermining education. New York, NY: Basic Books.

Resnick, L. B. (1987). Learning in school and out. Educational Researcher, 16(9), 13-54.

Ross, S. L. (2010). Transformative travel: An enjoyable way to foster radical change. ReVision, 32(1), 54-61.

Scarles, C. (2012). The photographed other: Interplays of agency in tourist photography in Cusco, Peru. Annals of Tourism Research, 39(2), 928-950.

Slattery, P. (2013). Curriculum development in the postmodern era: Teaching and learning in an age of accountability ( $3^{\text {rd }}$ ed.). New York, NY: Routledge.

Smiley, T., \& West, C. (2012). The rich and the rest of us: A poverty manifesto. New York, NY: Hay House.

Smith, J. J., \& Stovall, D. (2008). 'Coming home' to new homes and new schools: Critical race theory and the new politics of containment. Journal of Education Policy, 23(2), $135-152$.

Solórzano, D. G. (1997). Images and words that wound: Critical race theory, racial stereotyping, and teacher education. Teacher Education Quarterly, 24, 5-19. 
Solórzano, D. G., \& Yosso, T. J. (2009). Critical race methodology: Counter storytelling as an analytical framework for educational research. In E. Taylor, D. Gillborn, \& G. Ladson-Billings (Eds.), Foundations of critical race theory in education (pp. 131-147). New York, NY: Routledge.

Spielman, J. (2001). The family photography project: "We will just read what the pictures tell us." The Reading Teacher, 54(8), 762-770.

Steele, C. M. (2009). A threat in the air: How stereotypes shape intellectual identity and performance. In E. Taylor, D. Gillborn, \& G. Ladson-Billings (Eds.), Foundations of critical race theory in education (pp. 163-189). New York, NY: Routledge.

Stovall, D. (2013a). Against the politics of desperation: Educational justice, critical race theory, and Chicago school reform. Critical Studies in Education, 54(1), 33-43.

Stovall, D. (2013b, February). Globalizing the local: Justice as interrupting the grip of neoliberal urbanism. Keynote Address at the Globalization, Diversity and Education Conference, Vancouver, WA.

Sue, D. W., Capodilupo, C. M., Torino, G. C., Bucceri, J. M., Holder, A., Nadal, K. L., \& Esquilin, M. (2007). Racial microaggressions in everyday life: Implications for clinical practice. American Psychologist, 62(4), 271.

Tatum, B. D. (2009). Teaching white students about racism: The search for white allies and the restoration of hope. In E. Taylor, D. Gillborn, \& G. Ladson-Billings (Eds.), Foundations of critical race theory in education (pp. 277-290). New York, NY: Routledge. 
TRAVEL FOR TRANSFORMATION

Taylor, E. (2006). A critical race analysis of the achievement gap in the United States:

Politics, reality, and hope. Leadership and Policy in Schools, 5(1), 71-87.

Taylor, E. (2009). Critical race theory and interest convergence in the backlash against affirmative action: Washington State and Initiative 200. In E. Taylor, D. Gillborn, \& G. Ladson-Billings (Eds.), Foundations of critical race theory in education (pp. 117-129). New York, NY: Routledge.

Taylor, E. W. (2007). An update of transformative learning theory: A critical review of the empirical research (1999-2005). International Journal of Lifelong Education, 26(2), 173-191.

Taylor, E. W. (2008). Transformative learning theory. New Directions for Adult and Continuing Education, 119, 5-15.

Taylor, E. W., \& Cranton, P. (2012). Reflecting back and looking forward. In E. W. Taylor \& P. Cranton (Eds.), The handbook of transformative learning: Theory, research, and practice (pp. 555-573). San Francisco, CA: Jossey-Bass.

Taylor, E. W., \& Snyder M. J. (2012). A critical review of research on transformative learning theory, 2006-2010. In E. W. Taylor \& P. Cranton (Eds.), The handbook of transformative learning: Theory, research, and practice (pp. 37-55). San Francisco, CA: Jossey-Bass.

Taylor, K. B. (2008). Mapping the intricacies of young adults' developmental journey from socially prescribed to internally defined identities, relationships, and beliefs. Journal of College Student Development, 49(3), 215-234. 
Taylor, K. B., \& Elias, D. (2012). Transformative learning: A developmental perspective. In E. W. Taylor \& P. Cranton (Eds.), The handbook of transformative learning: Theory, research, and practice (pp. 147-161). San Francisco, CA: Jossey-Bass.

Theoharis, G. (2007). Social justice educational leaders and resistance: Toward a theory of social justice leadership. Educational Administration Quarterly, 43(2), 221258.

Tisdell, E. J. (2012). Themes and variations of transformational learning: Interdisciplinary perspectives on forms that transform. In E. W. Taylor \& P. Cranton (Eds.), The handbook of transformative learning: Theory, research, and practice (pp. 21-36). San Francisco, CA: Jossey-Bass.

Tuck, E., \& Yang, K.W. (2014). R-words: Refusing research. In D. Paris \& M. T. Winn (Eds.), Humanizing research: Decolonizing qualitative inquiry with youth and communities (pp. 223-248). Thousand Oaks, CA: Sage Publications.

Tushnet, M. V. (2009). The "we've done enough" theory of school desegregation. In E. Taylor, D. Gillborn, \& G. Ladson-Billings (Eds.), Foundations of critical race theory in education (pp. 99-108). New York, NY: Routledge.

Vogt, W. P., Gardner, D. C., \& Haeffele, L. M. (2012). When to use what research design. New York, NY: Guilford Press.

Willis, P. (2012). An existential approach to transformative learning. In E. Taylor \& P. Cranton (Eds.), The handbook of transformative learning: Theory, research, and practice (pp. 212-228). San Francisco, CA: Jossey-Bass. 
Writer, J. H. (2002). Terrorism in Native America: Interrogating the past, examining the present, and constructing a liberatory future. Anthropology and Education Quarterly, 33(3), 317-330. 


\section{Appendix A: Pre-/Post-Surveys and Permission for Use}

\section{Pre-Trip Evaluation and Expectations}

The information you provide on this evaluation will be used to improve this program in the future. Your responses are our major opportunity to receive feedback from you, so please answer the questions conscientiously. Just as on campus, your instructor should not be present when your group completes these evaluation forms. After all students have filled out the forms, one student should seal them in the manila envelope provided and return them to the Department of Modern Languages and Literatures Office. Thank you for your time and your help!

Program Name Dates

Your Major Classification Gender

Have you ever been overseas before? If so, how old were you? What was the purpose of that trip?

\section{Preparation Course/Program Orientation}

1. How well do you think that the pre-trip meetings have prepared you for your experience abroad? What do you think was the most valuable information from these meetings? What else do you wish you had known before the study-abroad experience?

2. What improvements would you suggest for the meetings?

\section{THE STUDY ABROAD PROGRAM}

\section{Program Housing}


What do you expect your housing arrangements will be like? Comment on the type of housing, meals, transportation, etc., as applicable.

\section{Program Transportation}

What do you think your travel and transportation will be like?

\section{Cultural Experience}

How do you think that the program can facilitate your understanding of the host culture? Comment on theatres, music, movies, business visits, social life, etc.

\section{Excursions}

Describe and comment on the excursions might expand your cultural understanding and how they relate to your course work.

\section{Finances}

1. How do you plan to finance your study abroad program? (check all that apply)

_ Personal funds Loans Family

support

_ Study Abroad Scholarship Other scholarships PSU financial aid

_ Other (what?)

2. What do you plan for your expenses (excluding program cost, tuition and fees, and books)?

\section{Goals and Expectations}

1. What are your goals for your study abroad experience? Do you feel you can meet these goals? 
GOALS MET? YES NO NOT CERTAIN

Academic Goals:

Cultural Goals:

Personal Goals:

2. What do you expect will be the best part of your experience with this program?

3. What do you expect will be the most challenging part of this international experience?

4. Do you have other general comments you would like to make?

\section{Post-Trip Evaluation}

The information you provide on this evaluation will be used to improve this program in the future. Your responses are our major opportunity to receive feedback from you, so please answer the questions conscientiously. Just as on campus, your instructor should not be present when your group completes these evaluation forms. After all students have filled out the forms, one student should seal them in the manila envelope provided and return them to the Department of Modern Languages and Literatures Office. Thank you for your time and your help!

Program Name Dates

Your Major Classification Gender

Have you ever been overseas before? If so, how old were you? What was the purpose of that trip?

\section{Preparation Course/Program Orientation}


1. How well did the pre-trip meetings prepare you for your experience abroad? What was most valuable of all the things you covered? What do you wish you had known before the program?

2. What improvements would you suggest for the meetings?

\section{THE STUDY ABROAD PROGRAM}

\section{Program Housing}

What were your housing arrangements? Comment on the type of housing, meals, transportation, etc., as applicable. Could they be improved? How?

\section{Program Transportation}

Were your air travel arrangements and ground travel arrangements satisfactory? How would you improve the travel arrangements for this program?

\section{Cultural Experience}

How did the program facilitate your understanding of the host culture? Comment on theatres, music, movies, business visits, social life, etc. Do you have any suggestions for improving cultural understanding?

\section{Excursions}

Describe and comment on the excursions and how they were related to your course work. Were they valuable? Why or why not? Do you have any suggestions for improvement?

\section{Finances}

1. How did you finance your study abroad program? (check all that apply) 
_ Personal funds

support
Loans

Family
Other scholarships

PSU financial

aid

Other (what?)

2. What were your expenses (excluding program cost, tuition and fees, and books)?

3. How much money would you recommend taking if you had it to do again?

4. What advice concerning finances would you give to future students on this program (please be specific, i.e., a daily or weekly budget)?

\section{Goals and Expectations}

1. What were your goals for your study abroad experience? Do you feel you met those goals?

GOALS MET?

YES

NO

NOT CERTAIN

Academic Goals:

Cultural Goals:

Personal Goals:

2. Would you recommend this program to other students? (If not, please explain.)

3. What was the best part of your experience with this program?

4. What was the most challenging part of this international experience?

5. If you were responsible for planning and implementing this program next year, what changes would you make? 
6. Other advice and general tips for students who are considering participating in this program in the future:

7. Would you be willing to help with next year's program (assisting with recruiting, publicity, and counseling? If so, please give us your name, address and phone number for the upcoming Fall semester, either below or on another page for confidentiality.

8. Do you have other comments you would like to make about this program in general?

\section{Permission to Use Surveys}

James,

The questionnaires are not copyrighted. My colleague (name omitted to protect confidentiality) created the document for one of her study abroad programs, and I adjusted it for mine. We use it in the department to have some kind of feedback, that is all. You may use the data that we collect from them, not to worry. I am going to have to adjust the question about them including their personal information if they are willing to tell future students about the program. The form is supposed to be anonymous. 


\section{Appendix B: Human Subjects Approval}

$\begin{array}{ll}\text { Post Otfle Box } 751 & \text { Por-725-2227 tel } \\ \text { Portland, Oregon } 97207-0751 & \begin{array}{l}503-725-8170 \text { fax } \\ \text { Human Subjects Research Review Committee } \\ \text { hsrrcelists.pdx.edu }\end{array}\end{array}$

Date: April 24, 2014

To: Susan Lenskj, James Gambrell

From: Karen Cellarius, HSRRC Chair

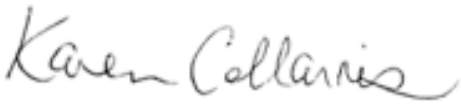

Re: HSRRC approval for your project titled, "Travel for Transformation: Pedagogy for the Privileged or Vehicle for Critical Socio-Cultural Transformation?" HSRRC Proposal \# 143031

Review Type: Exempt, Category 2

In accordance with your request, the Human Subjects Research Review Committee has reviewed your proposal referenced above for compliance with PSU and DHHS policies and regulations covering the protection of human subjects. The Committee is satisfied that your provisions for protecting the rights and welfare of all subjects participating in the research are adequate, and your project is approved. Please note the following requirements:

Approval: You are approved to conduct this research study after receipt of this approval letter; and the research must be conducted according to the plans and protocol submitted.

Changes to Protocol: Any changes in the proposed study, whether to procedures, survey instruments, consent forms or cover letters, must be outlined and submitted to the Committee immediately. The proposed changes cannot be implemented before they have been reviewed and approved by the Committee.

Adverse Reactions and/or Unanticipated Problems: If any adverse reactions or unanticipated problems occur as a result of this study, you are required to notify the Committee immediately. If the issue is serious, appockal max be withdraxn pending an inxestigation by the Committec.

Completion of Study: Please notify the Committee as soon as your research has been completed. Study records, including protocols and signed consent forms for each participant, must be kept by the investigator in a secure location for three years following completion of the study (or per any requirements specified by the project's funding agency).

If you have questions or concerns, please contact the Office of Research Integrity in the PSU RSP at hsrre@pdx.edu. 